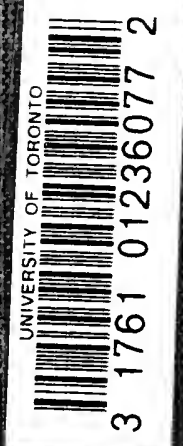

Sin 



<smiles>c1ccccc1</smiles>

$$
2^{b^{c}}
$$




\section{DIE WISSENSCHAFT}

SAMMLUNG

NATURWISSENSCHAFTLICHER UND MATHEMATISCHER

MONOGRAPHIEN

\section{Z E H N T E S HE F T}

D I E

\section{PSYCHISCHEN MASSMETHODEN}

$\mathrm{V} O \mathrm{~N}$

DR. G. F. LIPPS

PRIVATDOZENT DER PHILOSOPIIE AN DER UNIVERSITÄT LEIPZIG

MIT 6 EINGEDRUCKTEN ABBILDUNGEN

B R A U S C H W E G

DRUCK UND VERLAG VON FRIEDRICH VIEWEG UND SOHN

1906 


\title{
D IE
}

\section{PSYCHISCHEN MIASSMETHODEN}

V O N

\author{
Dr. G. F. LIPPS
}

PRIVATDOZENT DER PHILOSOPHIE AN DER UNIVERSITÄT LEIPZIG

MIT 6 EINGEDRUCKTEN ABBILDUNGEN

\section{B R A U N S CHWEIG}

DRUCK UND VERLAG VON FRIEDRICH VIEWEG UND SOHN 1906 


\section{Alle Rechte,}

namentlich dasjenige der Übersetzung in fremde Sprachen, vorbehalten.

Published December 20, 1905.

Privilege of Copyright in the United States reserved under the Act approved March 3, 1905 by Friedr. Vieweg \& Sohn, Braunschweig

\section{Germany.}

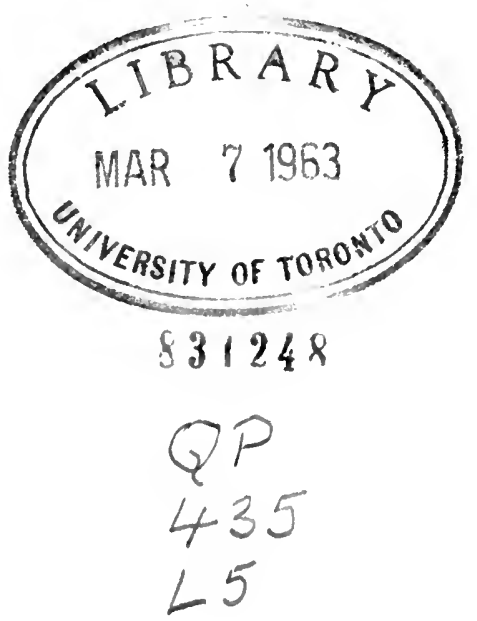




\section{0 R W 0 R T.}

Die psychischen Maßmethoden sind die auf der Verwendung von $\mathrm{Maß}$ und Zahl beruhenden Methoden der experimentellen Psychologie. Ihre Entwickelung setzt eine Verständigung über die Aufgabe der experimentellen Psychologie voraus. Diesem Zwecke dient der erste Abschnitt. Er führt zur Erkenntnis, daß die experimentelle Psychologie in der auf das psychologische Experiment gestützten Lelire von den Bewußtseinsinhalten besteht. Es gibt aber nicht etwa zwei Arten von Experimenten: alt hergebrachte naturwissenschaftliche und neu erfundene psychologische; es ist vielmehr bei jedem Experimente objektives Geschehen mit subjektivem Erleben verknüpft, so daß ebensowohl im naturwissenschaftlichen Interesse der Zusammenhang des objektiven Geschehens, wie auch im psychologischen Interesse die subjektive Auffassungs- und Beurteilungsweise unter der Herrschaft mannigfach abgeänderter Einflüsse erforscht werden kann.

Zwischen den Bewußtseinserscheinungen und den objektiven Zuständen und Vorgängen, die ihnen im Leibe des Menschen oder in der Außenwelt entsprechen, besteht aber kein kausaler, auf Äquivalenzbeziehungen beruhender $\mathrm{Zu}$ sammenhang, sondern nur eine größere oder geringere durch wechselnde Umstände bedingte Abhängigkeit. Die 
Empfindungen gehen nicht aus den Reizzuständen, in welche die Nerven durch Einwirkungen außerhalb oder innerhalb des Leibes versetzt werden, hervor wie Wärme aus mechanischer Arbeit. Ebensowenig verzehren sich die Gefühle, indem sie in Erregungen motorischer Nerven und somit in Bewegungen der Leibesorgane und in Handlungen sich umsetzen. Darum kann die Verwendung von Maß und Zahl bloß zur Bestimmung mehrfach bedingter Abhängigkeiten, nicht zur Ableitung eindeutiger Åquivalenzbeziehungen führen. Sie muß sich somit auf die im zweiten Abschnitt behandelte Wahrscheinlichkeitslehre und insbesondere auf das Bernoullische Theorem stützen.

Bei der Entwickelung der Maßmethoden, die sich an diese vorbereitenden Darlegungen anschließt, stellte ich mir die doppelte Aufgabe: einesteils zu zeigen, daß die von Fechner in Anlehnung an das gewöhnliche Fehlergesetz begründeten .und im Anschluß an ihn weiter ausgebildeten psychophysischen Maßmethoden unzureichend sind; und anderenteils den Weg anzugeben, auf dem man ohne Voraussetzung eines bestimmten Fehlergesetzes zu einer allen Bedürfnissen der experimentellen Psychologie genügenden Methode der $\mathrm{Maß}$ - und Abhängigkeitsbestimmung gelangt. Hierbei durfte nicht unbeachtet bleiben, daß einerseits die im naturwissenschaftlichen Gebiete vorliegenden Anfänge der experimentellen Psychologie schon über den Umkreis der Aufgaben, die Fechner in den "Elementen der Psychophysik" behandelt hat, hinausweisen, und daß andererseits die Beschränkung auf die sogenannte Psychophysik bei Fechner nicht durch psychologische, sondern durch naturphilosophische Interessen bedingt war. Darum muß der von Fechner eingenommene Standpunkt verlassen werden, wenn das Gesamtgebiet der experimentellen Psychologie einheitlich erfaßt werden soll. Dann 
erst ist es möglich, die auf der Verwendung von $\mathrm{Maß}$ und Zahl beruhenden Methoden so auszugestalten, daß die durch Wundt schon in den „Beiträgen zur Theorie der Sinneswahrnehmung" und in den „Vorlesungen über die Menschenund Tierseele" in die Psychologie eingeführte Untersuchung der Zeitrerbältnisse und des Verlaufs psychischer Vorgänge, sowie die Bestimmung des Wohlgefallens und Mißfallens in der durch Fechner begründeten experimentellen Ästhetik nicht minder als die traditionell bevorzugte Beziehung zwischen Reiz und Empfindung von ihnen beherrscht werden.

Leipzig, im Oktober 1905.

$$
\text { G. F. Lipps. }
$$





\section{INHALTSVERZEICHNIS.}

Erster Abschnitt.

Seite

Psychologie und Naturwissenschaft ............ 1

1. Die empirische und die philosophische Weltbetrachtung 1

2. Die Bewußtseinsinhalte . . . . . . . . . . . . 11

Zweiter Abschnitt.

Die Wahrscheinlichkeitslehre ............. 17

3. Gewißheit und Wahrscheinlichkeit . . . . . . . 17

4. Die Wahrscheinlichkeitsbestimmung . . . . . . . 23

Dritter Abschnitt.

Die Maßbestimmungen bei der Berücksichtigung subjektiver Faktoren im Bereiche der naturwissenschaftlichen

Forschung . . . . . . . . . . . . . 33

5. Die Beobachtungsfehler . . . . . . . . . . . . 33

6. Die Ungenauigkeit der Sinneswahrnehmung und die sonstigen subjektiven Faktoren . . . . . . . . 39

Vierter Abschnitt.

Die psychophysischen Maßmethoden . . . . . . . 46

7. Der naturphilosophische Standpunkt Fechners und das psychophysische Grundgesetz . . . . . . . 46

8. Das Maß der Empfindlichkeit . . . . . . . . . . . 49

9. Die Methode der eben merklichen Unterschiede . . . . 51

10. Die Methode der mittleren Fehler . . . . . . . . 59

11. Die Methode der richtigen und falschen Fälle . . . . 67

12. Die Methode der mittleren Abstufungen . . . . . 76

13. Die Beobachtungsreihen . . . . . . . . . . . . 80

14. Das Fehlergesetz . . . . . . . . . . . . . . . 89

15. Die Mittelwerte der Beobachtungsreihen . . . . . . 93 
Fünfter Abschnitt.

\section{Das psychische $\mathrm{MaB}$}

16. Die durch Fechner begründete Auffassungsweise des psychischen Maßes . . . . . . . . . . . 103

17. Ordnen und Messen . . . . . . . . . . . 107

\section{Sechster Abschnitt.}

Die Methoden der psychischen Abhängigkeitsbestimmang . . 115

18. Die Bestimmung des Grades der Abhängigkeit . . . 115

19. Der Typus der Beobachtungsreihe . . . . . . . . . 123

20. Die Zerlegung der Beobachtungsreihe in Komponenten und die Bestimmung der Unterschiedsschwelle . . 128

Anhang . . . . . . . . . . . . . . . 139

21. Die Berechnung der Mittelwerte. . . . . . . . 139

Literaturverzeichnis . . . . . . . . . . . . . . . . 144

Register . . . . . . . . . . . . . 149 
Erster Abschnitt.

\section{Psychologie und Naturwissenschaft.}

\section{Die empirische und die philosophische Weltbetrachtung.}

Jede Erkenntnis beruht auf der Betätigung des Denkens im Unterscheiden und Verknüpfen.

Ist nämlich im Gebiete der Erfahrungswissenschaft ein geschichtliches Ereignis oder eine Naturerscheinung, ein lebendes Wesen oder eine leblose Substanz zu untersuchen, so gilt es sowohl den vorhandenen Tatbestand festzustellen, als auch die an ihm und im Zusammenhange mit ihm sich vollziehenden Änderungen anzugeben. Die Feststellung eines Tatbestandes erfordert aber das Erfassen und Unterscheiden der aufzeigbaren, zur Charakterisierung geeigneten Besonderheiten; und die Beobachtung einer Veränderung führt dazu, die verschiedenen nebeneinander und nacheinander ablaufenden Zustände aufeinander zu beziehen und so miteinander zu verknüpfen. - Andererseits bedarf man im Gebiete der Mathematik und Logik grundlegender Definitionen, welche die Gegenstände der Untersuchung bestimmen und voneinander unterscheidbar machen, und zusammenhängender Systeme von Beziehungen, woraus die mathematischen und logischen Erkenntnisse sich ergeben.

Es bedarf indessen nicht erst des Hinweises auf eine aus einer Reihe zusammenhängender Denkakte bestehende Untersuchung. Schon der einzelne Denkakt läßt das Unterscheiden und Verknüpfen als die beiden Grundformen des Denkens hervortreten. Er findet in einem aus Subjekt und Prädikat bestehenden Urteile seinen Ausdruck. Der Vollzug des Urteils schließt

Lipps, Die psychischen Maßmethoden. 
aber ebensowohl die Unterscheidung wie auch die Verknüpfung der beiden Urteilsbestandteile ein.

Dies bestätigt sich, wenn man die einander entgegengesetzten Auffassungsweisen, die das Urteil bei verschiedenen Forschern erfahren hat, nebeneinander stellt.

So bezeichnet beispielsweise Sigwart ${ }^{1}$ ) das Urteil als einen Denkakt, der jedenfalls voraussetzt, daß zwei unterschiedene Vorstellungen dem Urteilenden gegenwärtig sind, die mit Bewußtsein in Eins gesetzt werden. Dabei erinnert er an den Ausspruch des Aristoteles ${ }^{2}$ ), daß da, wo Irrtum oder Wahrheit vorkommen kann (und dies ist beim Urteil der Fall), eine Ver-

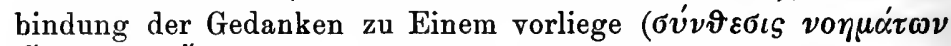

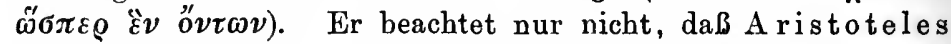
weiterhin bemerkt, man könne auch alles eine Teilung nennen, wodurch nach meinem Dafürhalten die unterscheidende Funktion des Denkens ihre Anerkennung findet. Diese letztere wird hingegen durch $W \mathrm{undt}^{3}$ ) hervorgehoben, wenn er das Wahrnehmungsurteil als die Zerlegung einer Gesamtrorstellung in ihre Bestandteile definiert und auch in den Urteilen, die ein abstraktes Denken voraussetzen, die Zerlegung eines Gedankens in seine begrifflichen Bestandteile erblickt.

Vereinigt man beide Auffassungsweisen, so findet man, daß in der Tat im Unterscheiden und Verknüpfen das Wesen des Urteils und somit des Denkens überhaupt sich offenbart.

Eine Unterscheidung kann nun durch andere Unterscheidungen, eine Beziehung durch andere Beziehungen bedingt sein oder selbständig existieren. Es treten so den für sich bestehenden Unterscheidungen oder Beziehungen Systeme zusammengehöriger Unterscheidungen oder Beziehungen gegenüber.

$\mathrm{Es}_{\mathrm{s}}$ ist aber auch möglich, daß einmal Beziehungen die Ausführung von Unterscheidungen voraussetzen und Unterscheidungen erst durch den Vollzug von Beziehungen möglich werden, und daß ein andermal Beziehungen unabhängig von Unterscheidungen und Unterscheidungen unabhängig von Beziehungen ausfübrbar sind.

1) Logik 1, 25, 63 (1889). 2. Aufl.

2) De anima 3, 6 .

3) Logik 1, 156, 158 (1893). 2. Aufl. 
In der Tat setzen die Beziehungen der Ähnlichkeit und des Gegensatzes zwischen den Farbenempfindungen die Unterscheidung der Farben voraus: der Farbenblinde weiß nichts von Farbenverwandtschaft und von Farbenkontrast. Man kann hingegen Farben unterscheiden, ohne den Grad ihrer Ähnlichkeit und ihres Gegensatzes zu beachten. Entsprechendes gilt für alle anderen Sinnesgebiete. Die Unterscheidung der Empfindungen ermöglicht erst die Ordnung derselben auf Grund der Beziehungen der Ähnlichkeit und des Gegensatzes; man bedarf aber nicht etwa der Einsicht in diese Beziehungen, um die einzelnen Empfindungen unterscheiden zu können.

Andererseits kann man zwei mit entgegengesetzten elektrischen Ladungen versehene Konduktoren erst dann hinsichtlich ihres elektrischen Zustandes unterscheiden, wenn man sie zu anderen elektrischen Substanzen in Beziehung setzt und die eintretenden Veränderungen beobachtet. In ähnlicher Weise ist überhaupt die objektive Unterscheidung der physikalischen $\mathrm{Zu}$ stände und der chemischen Eigenschaften der Substanzen an die Beobachtung ihrer Wirkungsweise gebunden. Demnach sind hier die Beziehungen das ursprünglich und schlechthin Bestehende und eine das Wesen der Substanzen treffende Unterscheidung ist erst auf Grund jener Beziehungen, die sich in den beobachteten Einwirkungen und Veränderungen kundgeben, möglich.

Hieraus erhellt, daß es ebensowohl gegebene Unterscheidungen wie auch gegebene Beziehungen sind, in denen sich die Welt dem Denken darbietet.

Die gegebenen Unterscheidungen sind, wie die angeführten Beispiele lehren, als Bewußtseinsinhalte $\mathrm{zu}$ bezeichnen. Sie können in Empfindungen und Vorstellungen einerseits, in einfache und zusammengesetzte Gefühle andererseits getrennt werden je nachdem es sich um das Unterscheiden objektiv existierender Dinge oder um Kennzeichen für verschiedene subjektive Zustände handelt. Es kommt indessen hier nicht darauf an, eine vollständige Klassifikation zu geben. Das Wesentliche ist vielmehr, daß die Bewußtseinsinhalte ihre klare und unzweideutige Definition erhalten, indem sie auf ursprünglich und schlechthin bestehende Unterscheidungen zurückgeführt werden, die ihrerseits nach Ausweis der Erfahrung keine weitere Zurückführung ge- 
statten. Die Lehre von den Bewußtseinsinhalten muß folglich als ein besonderer selbständiger Teil der Erfahrungswissenschaft anerkannt werden.

Die gegebenen Verknüpfungen hingegen weisen auf Objekte hin, die aufeinander wirken und sich verändern. Diese Objekte gewinnen ihre wesentlichen Merkmale durch die Art und Weise, wie sie Wirkungen ausüben und Veränderungen erleiden, und sind demgemä $\hat{B}$ in entwickelungsfähige, lebende Wesen und in leblose, lediglich mit physikalischen Zuständen und chemischen Eigenschaften behaftete Substanzen zu unterscheiden. Die Lehre von der objektiv existierenden Welt bietet somit den anderen, zu der Lehre von den Bewußtseinsinhalten hinzutretenden Teil der Erfahrungswissenschaft dar.

Wenn aber die Bewußtseinsinhalte bloß als Unterscheidungen und die objektiv existierenden Dinge nur als Träger von Beziehungen zur Geltung kommen, so darf nicht außer acht gelassen werden, daß weder die Bewußtseinsinhalte noch die wirkenden und sich verändernden Objekte für sich allein existieren. Sie bilden vielmehr in tatsächlich untrennbarer Zusammengebörigkeit die eine wirklich bestehende Welt, die nur für das Denken und auf Grund des Denkens in eine Welt der Bewußtseinsinhalte und in eine objektiv existierende Welt sich scheiden läßt.

Dieses unmittelbare Zusammenbestehen von Bewußtseinsinhalten und objektiv existierenden Dingen verleiht der empirischen Weltbetrachtung ihr charakteristisches Gepräge. Es kann in jeder Form als Ausgangspunkt für eine Untersuchung dienen, die dem einen oder dem anderen Teile der Erfahrungswissenschaft angehört.

Soll jedoch ein alle Erkenntnisse zusammenfassendes Weltbild hergestellt werden, so muß ein Ausgangspunkt gefunden werden, der die Zusammenfassung möglich macht. Die philosophische Weltbetrachtung tritt dann an Stelle der empirischen.

Ist man sich darüber klar, daß die gegebene Wirkiiichkeit lediglich dem Denken gegeben ist, und daß nur das Denken zu einer Erkenntnis führt, so kann es nicht zweifelhaft sein, daß die Untersuchung des Denkens den Ausgangspunkt für die philosophische Weltbetrachtung bilden muß. Dann wird man aber 
durch die Betrachtung der Denktätigkeit im einzelnen Urteilsakte und in zusammenhängender wissenschaftlicher Untersuchung dazu geführt, das Unterscheiden und das Beziehen oder Verknüpfen als die Grundformen des Denkens anzuerkennen, so daß sich auch die gegebene Wirklichkeit als ein Zusammen rons gegebenen Unterscheidungen oder Bewußtseinsinhalten und gegebenen Beziehungen oder objektiv existierenden Dingen darbietet. Auf diese Weise fíndet vom philosophischen Standpunkte aus die Erfahrungswissenschaft in ihren beiden Teilen, als Lehre vom Bewußtsein und vom objektiv existierenden Sein, ihre sichere Begründung. Insbesondere wird uns die Möglichkeit gegeben, das Wesen der Bewußtseinsinhalte klar zu erfassen und die bei ihrer Untersuchung anwendbaren Methoden einwandfrei aus sicheren Grundsätzen zu entwickeln.

Fehlt aber diese Einsicht, so wird man, statt vom Denken auszugehen, nach einer überempirischen Wirklichkeit suchen, die den Zugang zu der empirisch gegebenen Welt eröffnet. Dann wird man auch das Denken als eine dieser Welt angehörende Erscheinung auffassen, die ebenso wie das sonstige Weltgeschehen ihre Ableitung finden muß. Will man andererseits nur die Erfahrung gelten lassen, so besteht die Gefahr, daß man das Gegebene auf die im Bewußtsein unmittelbar vorliegenden Unterscheidungen reduziert und so nur Koexistenzen und Successionen von Bewußtseinsinhalten an Stelle der wirklichen Welt annimmt. - Es tritt so eine einseitige, von einer überempirischen Wirklichkeit ausgehende Weltbetrachtung einer nicht minder einseitigen, bloß Bewußtseinsinhalte als gegeben anerkennenden empirischen Weltbetrachtung gegenüber.

Da sich hiermit die von uns vollzogene Trennung der Erfahrungswissenschaft in die Lehre vom Bewußtsein und vom objektiven Sein nicht verträgt, so ist es notwendig, auf Grund der Geschichte der Philosophie in Kürze zu zeigen, wie der Widerstreit der beiden einseitigen Betrachtungsweisen schließlich zu der hier vertretenen, die empirische Auffassungsweise ergänzenden und vollendenden philosophischen Weltbetrachtung hinführt.

Nach einer überempirischen Wirklichkeit haben schon die ionischen Naturphilosophen gesucht. Sie gelangten zu der Ansicht, daß ein lebendiger, zeugungskräftiger Urstoff (das Wasser, 
das Unbegrenzte, die Luft) der Natur und dem Naturgeschehen zugrunde liege und die Ursache alles Lebens und aller Bewegung sei. - Die Philosophen hingegen, die dem Bunde der Pythagoreer angehörten, haben erkannt, daß alle Erscheinungen nach Zahlen geordnet seien, daß insbesondere die Harmonie der Töne auf Zahlenverhältnissen beruhe und daß die Zahlen auch die geometrischen Figuren beherrschen. So kommen sie zu der grundlegenden Lehre, daß die Zahl das Wesen aller Dinge sei, und daß alles aus Zahlen bestehe. Der Urgrund der Welt ist somit die substanziell existierende Zahl. - Die Lehre der Eleaten ferner gipfelt in der Erkenntnis, daß alles was ist seinem Wesen nach dasselbe ist. Jeder Unterschied, der als ein räumliches Nebeneinander oder als ein zeitliches Nacheinander durch die Sinne wahrgenommen wird, ist darum ein wesenloser Schein, dem keine wahre Existenz zukommt. Denn nur das Seiende, das ohne Anfang und ohne Ende und ohne Veränderung ist, existiert wirklich in unteilbarer und unbeweglicher Einheit. - Heraklit dagegen findet in der Welt kein beharrendes Sein, sondern unablässigen Wechsel, in dem sich das allem Geschehen zugrunde liegende Wesen offenbart. - Demokrit endlich nimmt als ursprüngliches Sein die unveränderlichen Atome in unbegrenzter Anzahl und verschiedener räumlicher Gestalt an, die im Raume fallen, aufeinander prallen und so unzählige nebeneinander und nacheinander entstehende Welten bilden, in denen alles - auch die Bewußtseinserscheinungen und das Denken - auf der Bewegung der Atome beruht.

Der Gegensatz zu diesem Suchen nach einem in der Erfahrung nicht gegebenen Ausgangspunkte machte sich in der griechischen Philosophie zum ersten Male dann bemerklich, als Anaxagoras dem chaotischen Zustande, in dem alle Stoffe

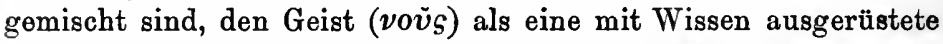
und zu zweckmäßigem Handeln befähigte Macht gegenübergestellt hatte. Hierdurch sollte zunächst die Zweckmäßigkeit des Naturgeschehens, die aus dem mechanischen Druck und Stoß der Atome Demokrits nicht wohl ableitbar war, ihre Erklärung finden. Da aber der vors vor allem in den Handlungen des Menschen sich geltend macht, so wurde dem philosophischen Denken eine neue, dem Menschen zugewandte, der Natur zunächst abgewandte Richtung gegeben. Sie fand insbesondere in dem 
Sophisten Protagora s ihren Vertreter, der nicht von der äußeren Natur, sondern von dem die Natur wahrnehmenden Menschen ausging. Er stellte den Grundsatz auf, daß der Mensch das Maß aller Dinge sei und meinte damit, $\mathrm{da} B$ es von unserem Empfinden abhänge, wie uns die Dinge erscheinen, und daß außer den Empfindungen uns nichts gegeben sei.

Dieser Ansatz fand indessen keine Weiterbildung, da durch Sokrates die Untersuchung der sittlichen Persönlichkeit und als das hierbei nicht zu entbehrende Hilfsmittel die Methode der Begriffsbestimimung in den Vordergrund gestellt wurde. Der Begriff ist aber von der zum Begriffe gehörenden Wahrnehmung verschieden. Darum löste Platon den Begriff von der Erscheinung los und faßte ihn als eine für sich existierende Idee auf. So entdeckte er in den Ideen eine neue, unsichtbare, unkörperliche Welt, die Welt des wahren Seins, aus der die gegebene Welt des Werdens abgeleitet werden sollte. Aristoteles dagegen faßte die Ideen nicht als Urbilder oder Vorbilder auf, die ein in sich abgeschlossenes und auf sich beruhendes Dasein führen, sondern als treibende, bestimmten Zielen zustrebende, in der Materie wirkende Kräfte. Erst in der Zeit des Niederganges der antiken Philosophie, bei den Epikureern und Skeptikern, wurde der von den Sophisten zuerst beachtete Inhalt der unmittelbaren sinnlichen Wahrnehmung in allerdings unwirksamer Weise von neuem hervorgehoben.

Weit wirkungsvoller tritt indessen der Widerstreit beider Auffassungsweisen in der neueren Philosophie zutage.

Descartes findet zwar im Denken den unanfechtbaren Ausgangspunkt für alles Erkennen. Da er aber das Denken in vermeintlicher substanzieller Existenz der ausgedehnten Körperwelt gegenüberstellt, sieht er sich zu der Annahme zweier, der Erfahrungswelt zugrunde liegender Substanzen, der denkenden und der ausgedehnten Substanz, genötigt, die wechselweise aufeinander einwirken. Diese Dualität vermeidet einerseits Spinoza, indem er Denken und Ausdehnung als zusammenbestehende und zusammengehörige Attribute der einen, alles Sein in sich schließenden Substanz auffaßte; und andererseits Leibniz, indem er das Wesen der denkenden Geister und der ausgedehnten Körper in die einfachen, ursprünglichen, ewigen Kräfte setzte, die als in sich abgeschlossene Einheiten oder Mo- 
naden mit der Fähigkeit, Vorstellungen zu haben und Wirkungen auszuüben existieren. Es bleibt jedoch sowohl in der absoluten Substanz Spinozas, wie auch in den Monaden Leibniz' der überempirische Ausgangspunkt der Weltbetrachtung bestehen.

Den Gegensatz hierzu bildet die englische Erfahrungsphilosophie, deren hervorragendster Vertreter David $\mathrm{Hume}$ ist. Seine schon von Locke aufgestellte und durch Berkeley weitergebildete Grundauffassung ist die, daß alle Erkenntnis a uf Erfahrung beruhe und daß alle Erfahrung in Vorstellungen bestehe. "Man richte seine Aufmerksamkeit so intensiv als möglich auf die Welt außerhalb seiner selbst, man dringe mit seiner Einbildungskraft bis zum Himmel oder bis an die äußersten Grenzen des Weltalls; man gelangt doch niemals einen Schritt weit über sich selbst hinaus, nie vermag man mit seiner Vorstellung eine Art der Existenz zu erfassen, die hinausginge über das Dasein der Perzeptionen, welche in dieser engen Sphäre des eigenen Bewußtseins aufgetreten sind. Dies ist das Universum der Einbildungskraft; wir haben keine Vorstellung, die nicht darin ihr Dasein hätte" ${ }^{1}$ ). Die Welt der Bewußtseinsinhalte (der Perzeptionen) bildet somit den alleinigen Ausgangspunkt für die Erkenntnis. Darum kann man bloß von einem Glauben an die Existenz der Körper reden und den Gründen für diesen Glauben nachforschen, die von $\mathrm{Hume}$ in der Kohärenz und der Beständigkeit gewisser Eindrücke gefunden werden.

Den Zwiespalt zwischen der Einschränkung der Erfahrungswelt auf bloße Unterscheidungen und der Überschreitung aller Erfahrung durch die Konstruktion einer transzendenten Wirklichkeit hat indessen Kant durch seine Vernunftkritik überwunden. Er stellte die Frage nach der Möglichkeit von Erkenntnissen, die nicht aus der Erfahrung stammen, sondern durch die Form unserer Anschauung und unseres Denkens bedingt sind, und kam hierdurch zu der Einsicht, daß zwar keine alle Erfahrung übersteigende Wirklichkeit durch das Denken erkannt werden könne, daß jedoch alles, was wir in Erfahrung bringen und aus der Erfahrung ableiten, an Formen gebunden sei, die in

1) David Humes Traktat über die menschliche Natur (herausgegeben von Th. Lipps); Tl. II, Abschn. 6, S. 91. 
der Beschaffenheit unseres Denkens ihren Grund haben. Darum ist es möglich, durch die Erforschung jener Formen eine Einsicht in die Bedıngungen des Erkennens zu gewinnen und eine Theorie des Erkennens aufzustellen. So gelangte Kant zu der von der Untersuchung des Denkens ausgebenden philosophischen Weltbetrachtung.

Daß aber nun an Stelle der reinen Anschauungsformen und der reinen Verstandsbegriffe Kants hier das Unterscheiden und Verknüpfen als die beiden Grundformen des Denkens zugrunde gelegt werden, ist nicht nur im Hinblick auf die bereits hervorgehobene Betätigung des Denkens im Urteilen und in zusammenhängender wissenschaftlicher Untersuchung, sondern auch mit Rücksicht auf die im Verlaufe des 19. Jahrhunderts eingetretene Entwickelung der Erfahrungswissenschaft geboten.

Durch den Satz von der Erhaltung der Energie ist nämlich für die moderne Physik und Chemie die objektiv existierende Welt in der Tat zu einem Systeme gegebener Beziehungen oder Verknüpfungen geworden: Aus Wasser wird Knallgas, aus Knallgas Wasser, - dies bedeutet nicht bloß, daß die Vorstellungen vou Wasser und von Knallgas aufeinander folgen. Denn die Verknüpfung, in der die Äquivalenz beider Stoffe ihren Ausdruck findet, ist keine Succession. So handelt es sich auch bei dem Satze, daß aus Bewegung Wärme und aus Wärme Bewegung wird, nicht um die von jeher bekannte Koëxistenz und Succession der beiden Zustände, sondern um die in der Äquivalenz von Bewegungsenergie und Wärme bestehende Beziehung.

Nicht minder deutlich tritt die Tatsache hervor, daß die moderne Psychologie, sofern sie als experimentelle Psychologie eine Analyse des Bewußtseins erstrebt, auf der unterscheidenden Funktion des Denkens beruht.

Dieser Einsicht steht zunächst entgegen, daß die Psychologie, ebenso wie die Naturwissenschaft, ursprünglich nicht von einer klaren philosophischen Auffassung der Grundlagen des Erkennens, sondern von ungeklärten, empirischen Betrachtungen ausgegangen ist und diesen Urzustand noch nicht in gleichem Maße, wie die moderne Physik und Chemie, überwunden hat. Für die populäre Auffassung ist nämlich der beseelte Mensch ebenso sehr ein wollendes und handelndes wie auch ein mit Bewußtsein begabtes 
Wesen, so daß die Psychologie als die Lehre rom beseelten Menschen zwei prinzipiell verschiedene Gebiete des Erkennens in unkritischer Vermengung umfaßt: die Lehre von dem innerhalb der objektiv existierenden Welt wollenden und handelnden Menschen, die als ein Bestandteil der allgemeinen Entwickelungslebre zu der Lehre vom objektiven Sein und Werden gehört, und die Lehre vom Bewußtsein.

Die ursprüngliche Selbständigkeit der letzteren tritt aber in der modernen Psychologie infolge der Einführung des psychologischen Experimentes unverkennbar zutage. Denn das psychologische Experiment hat nicht etwa die Seele des Menschen zum Gegenstande, während das naturwissenschaftliche Experiment an den Objekten der äußeren Welt ausgeführt wird. Alles Experimentieren besteht vielmehr in einer willkürlichen Beeinflussung des Naturgeschehens und in der Beobachtung desselben, so daß objektive und sabjektive Faktoren untrennbar miteinander verwoben sind. Es kann sich folglich nur darum handeln, entweder das objektive Geschehen festzustellen oder die subjektiven Faktoren unter mannigfach abgeänderten Bedingungen zu untersuchen. Im letzteren Falle wird es zum psychologischen Experiment, das — wie Wundt sagt ${ }^{1}$ ) - „die um der schwankenden Beschaffenheit der Erscheinungen willen von besonderen Schwierigkeiten umgebene Analyse der Bewußtseinsvorgänge eigentlich überhaupterst möglich macht".

Demgemäß kann das vom psychologischen Experimente beherrschte Gebiet der Psychologie lediglich in der Untersuchung der subjektiven Auffassung des objektiven Geschehens bestehen, wonach es sich als die von der Erfahrung ausgehende Lehre von den BewuBtseinsinhalten darbietet und in der Funktion des Unterscheidens seine selbständige Grundlage findet.

Auf Grund dieser Einsicht kann das Wesen der Bewußtseinsinhalte klargestellt und der Weg, auf dem man zu einer auf das psychologische Experiment gestützten Untersuchung der Bewußtseinsinhalte gelangt, gezeigt werden.

1) Die Philosophie im Beginn des 20. Jahrhunderts. Festschrift für Kuno Fischer, Bd. I, Psychologie S. 40. 


\section{Die Bewubtseinsinhalte.}

Da eine Unterscheidung nicht in eine Verknüpfung und eine Verknüpfung nicht in eine Unterscheidung umgedeutet werden kann, so läßt sich auch ein Bewußtseinsinhalt nicht auf objektives Geschehen und das letztere nicht auf ein Zusammen und Nacheinander von Bewußtseinsinhalten zurückführen. Es ist somit nur ein Zusammenbestehen von gewissen Bewußtseinsinhalten einerseits und gewissen Zuständen und Vorgängen der objektiven Welt andererseits feststellbar.

Dieses Zusammenbestehen kann, wenn ein Bedürfnis vorliegt, als Parallelismus und zwar als psychophysischer Parallelismus bezeichnet werden, wofern man unter den Bewußtseinsinhalten das Psychische, unter dem objektiv Existierenden das Physische versteht. Der in dieser Form sich darbietende psychophysische Parallelismus hat indessen mit der Annahme oder der Verwerfung der Wechselwirkung zwischen einem Seelenwesen und einem von der Seele bewohnten und beherrschten Leibe nichts zu tun; denn die Frage nach der Existenz der Seele und ihrer etwaigen Wirkungsweise kommt hier so wenig wie bei der Lösung eines mathematischen Problems oder der Ausführung eines physikalischen Experimentes in Betracht. Es handelt sich vielmebr lediglich um das Hervorheben der Grundtatsache, auf der die experimentelle Psychologie in ihrem ganzen Umfange beruht. Darum spricht Wundt von dem "heuristischen Prinzip des psychophysischen Parallelismus", das bei ihm in folgendem Satze seinen Ausdruck findet ${ }^{1}$ ): „Überall, wo regelmäßige Beziehungen zwischen psychischen und physischen Erscheinungen bestehen, sind beide weder identisch noch ineinander transformierbar, denn sie sind an sich unvergleichbar; aber sie sind einander in der Weise zugeordnet, daß gewissen psychischen gewisse physische Vorgänge regelmäßig entsprechen, oder, wie man sich bildlich ausdrückt, daß beide einander parallel gehen."

Eine derartige Zuordnung von Bewußtseinsinhalten zu objektiven Zuständen oder Vorgängen hat bereits - wie eine sagenhafte Erzählung berichtet2) - Pythagoras vollzogen.

1) Grundzüge der physiolog. Psychologie 3, 769 (1903). 5. Aufl.

2) Poggendorff, Geschichte der Physik, 1879, S. 30. 
Beim Vorübergehen vor einer Schmiede hörte er im Zusammenklang der Hämmer die Quarte, die Quinte und die Oktave. Da er nun im Gewichte der Hämmer den Grund der Tonintervalle vermutete, so ging er hinein, um die Hämmer zu wiegen, und soll tatsächlich bestimmte Gewichtsverhältnisse, die den gehörten Intervallen entsprachen, gefunden haben.

Auch hat sich bald die Tatsache, daß keine auf Äquivalenz gegründete Kausalität besteht, bemerklich gemacht. Schon in Plat ons Theätet wird eine auf Heraklit zurückweisende, dem Sophisten Protagoras zugeschriebene Lehre auseinander gesetzt, nach welcher die Sinneswahrnehmungen durch die Einwirkung äußerer Dinge und durch die Gegenwirkung der Sinnesorgane entstehen. Es wird so anerkannt, daß die Wahrnehmungen nicht einfach aus den wahrgenommenen Gegenständen hervorgehen.

Dieser Erkenntnis verleiht Aristoteles einen noch bestimmteren und reineren Ausdruck. Er definiert 1) die Sinneswahrnehmung als eine Veränderung des Wahrnehmenden durch das Wahrgenommene. Wie das Wachs das Zeichen des Siegels aufnimmt, nicht aber das Eisen oder Gold des Siegels, so erfaßt auch der Sinn die wahrnehmbare Form ohne den Stoff. Die selbständige Bedeutung des Sinnesorgans, das von sich aus die Fähigkeit zur Wahrnehmung haben muß, kommt so vollständig zur Geltung.

In den "Problemen des Aristoteles" 2 ) wird ferner bereits der Einfluß früherer Wahrnehmungen auf die Beurteilung eines Sinneseindrucks zur Erklärung von Sinnestäuschungen benutzt. Dort wird nämlich die Frage aufgeworfen, warum ein einzelner Gegenstand doppelt gesehen wird, wenn man die Augen in eine gewisse Stellung zueinander bringt, und warum eine kleine Kugel doppelt erscheint, falls sie von den übereinander gekreuzten Fingern berührt oder besser hin und her gerollt wird. Und die Antwort weist in beiden Fällen darauf hin, daß bei gewöhnlicher Augenstellung und bei der üblichen Haltung der Finger zwei Objekte vorhanden sein müßten, wenn die nämliche Eregung zustande kommen sollte, so daß man auch bei der abnormen Stellung der Augen und der gekreuzten Haltung der Finger zwei Empfindungen zu haben glaube.

1) De anima $2,5$.

) Problemata 31, 11, 18; 35, 10. 
Außerdem haben die Skeptiker die Unsicherheit der Sinneswahrnehmung auf Grund unmittelbar vorher stattgehabter Erregungen und gleichzeitig stattfindender Nebenerregungen erkannt. Sextus Empiricus, der (gegen Ende des zweiten Jahrhunderts n. Chr.) in seinen Schriften eine vollständige Darstellung der skeptischen Philosophie gibt, erwähnt in den Pyrrhoneischen Grundzügen ${ }^{1}$ ), daß „derselbe Wein denen, welche vorher Datteln oder Feigen gegessen haben, säuerlich scheint, denen aber, welche Walnüsse oder Kichererbsen zu sich genommen, angenehm zu sein scheint, und daß die (lauwarme) Mittelhalle des Badehauses die von außen Eintretenden erwärmt, die Hinausgehenden aber abkühlt, wenn sie darin verweilen". Er weiø auch, daß wir wegen der zu den Einwirkungen der wahrgenommenen Gegenstände hinzutretenden Beimischungen in den Sinneswahrnehmungen nicht ein unmittelbares Abbild der Gegenstände haben. "Deswegen sehen die Gelbsüchtigen alles gelb, die mit blutunterlaufenen Augen blutig."

Noch klarer und deutlicher tritt jedoch in der neueren Philosophie die Einsicht zutage, daß keine Äquivalenz zwischen den subjektiven Wahrnehmungen und der objektiven Beschaffenheit des Wahrgenommenen besteht.

Bei Descartes ist es die mit dem Leibe in Wechselwirkung stehende Seele, die wahrnimmt. Sie hat ihre besondere Natur, derzufolge durch die Finwirkungen äußerer Objekte auf die Sinnesorgane und das Gehirn bestimmte Vorstellungen erregt worden, ohne daß dieselben irgend welche Ähnlichkeit mit den sie verursachenden materiellen Vorgängen hätten. Dazu kommt noch die im Denken bestehende eigene Tätigkeit der Seele, durch welche beispielsweise die Entfernung eines Gegenstandes auf Grund des Winkels, den die Sehachsen der beiden, den Gegenstand fixierenden Augen miteinander bilden, erschlossen werde.

Daß indessen diese eigene Tätigkeit der Seele nicht in der Konstruktion von Sehachsen und Winkeln bestehen könne, sondern sich auf wirkliche, in der Erfahrung gegebene Vorstellungen stũtzen müsse, hat Berkeley in seiner "Neuen Theorie des Sehens" gelehrt. Da nun die Gesichtsvorstellungen nichts von

') Erstes Buch, Kap. 14, S. 110, 126; nach der Übersetzung von Pappenheim. 
der Entfernung, Größe und Lage der Objekte enthalten, so können sie nur durch Assoziation von Tastrorstellungen mit den Farbenund Helligkeitsempfindungen des Gesichtssinnes bedingt und auf Grund einer langen Erfahrung erworben sein. So ist denn das, was wir als Objekt der äußeren Welt wahrnehmen, vielmehr ein Ergebnis der in der Persönlichkeit des Beobachters aufgebäuften Erfahrung als ein Erzeugnis der unmittelbaren, von außen kommenden Einwirkung auf die Sinnesorgane.

Es wird somit durch die in alter und neuerer Zeit binsichtlich der Sinneswahrnehmung gewonnene Einsicht bestätigt, daß in der Tat bloß ein mannigfach bedingtes Zusammenbestehen objektiver Zustände und Vorgänge und ihrer subjektiven Wahrnehmung feststellbar ist.

Die Bedingtheit ist aber als eine vierfache zu bezeichnen: Zunächst und in erster Linie besteht eine tatsächliche,

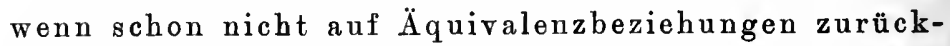
führbare Abhängigkeit gewisser Wahrnehmungen von den wahrgenommenen Objekten. Wäre dies nicht der Fall, so könnten die Wahrnehmungen nicht als Hinweise auf Objekte, sondern blo@ als Merkzeichen für die wechselnden Zustände des beobachtenden Subjekts Geltung gewinnen. Sodann ist der Einfluß gleichzeitiger Eindrücke - der Beimischuhgen, wie die Skeptiker sagten - und ferner die gleichfalls von den Skeptikern beachtete Bedeutung unmittelbar vorhergegangener Erregungen anzuerkennen. Es ist schließlich die Beeinflussung durch frühere Erlebnisse hervorzuheben, wie sie in der Gewöhnung an bestimmte Erlebnisse und in der Anhäufung von Erfahrungen in den Problemen des Aristoteles und namentlich in Berkeleys Theorie des Sehens zutage tritt.

Zu dieser Erkenntnis gelangt man indessen ohne weiteres, wenn man erwägt, daß die Bewußtseinsinhalte als Unterscheidungen aufzufassen sind. Dann ist nämlich in erster Linie ein unterscheidendes Subjekt vorauszusetzen, das bereits Erlebnisse gehabt und angesammelt haben muß, wenn es überhaupt imstande sein soll, an dem Erlebten das eine vom anderen zu unterscheiden. Jede Besonderung innerbalb der Gesamtheit des Erlebten ist darum ohne weiteres eben durch jene Gesamtheit, die in der Person des unterscheidenden Subjekts vorliegt, bedingt. 
Sind nun Unterscheidungen ausgeführt worden, so wird jede neue Unterscheidung von der unmittelbar zuvor vollzogenen sich abheben und so durch die letztere beeinflußt sein. Es werden aber in entsprechender Weise alle Unterscheidungen, die innerhalb eines jeweils abgesonderten Komplexes vorliegen und als gleichzeitige Unterscheidungen sich darbieten, sich wechselweise beeinflussen. Nur die Zugehörigkeit zu objektiven Zuständen und Vorgängen kann auf diesem Wege sich nicht ergeben, eben weil diese Zugehörigkeit nicht in der Funktion des Unterscheidens begründet ist, sondern darauf beruht, $\mathrm{daß}$ die gegebene Wirklichkeit sich nicht nur in Unterscheidungen, sondern auch in Beziehungen oder Verknüpfungen darbietet.

Die von der Erfahrung ausgehende, auf das psychologische Experiment sich stützende Lehre von den Bewußtseinsinhalten ist aber, wie wir (S. 10) erkannt haben, die experimentelle Psychologie. Ihre Aufgabe ist demnacb, der vierfachen Bedingtheit der Bewußtseinsinhalte entsprechend, gleichfalls als eine vierfache zu bezeichnen:

Zuvörderst ist der unmittelbare Zusammenhang der im Bewußtsein vollziehbaren Unterscheidungen mit dem zugrunde liegenden objektiven Geschehen zu erforschen. Es ist zweitens die hierbei sich kundgebende Beeinflussung durch gleichzeitige und drittens die Bedingtheit durch unmittelbar vorhergegangene Inhalte des Bewußtseins festzustellen. Es ist viertens die Gesamtheit der Unterscheidungen, die der Beobachter in seinem bisherigen Leben vollzogen hat und die für die gegenwärtig auszuführenden Unterscheidungen die Voraussetzung bilden (die in der Persönlichkeit des Beobachters vorliegende Gesamtheit der in Betracht kommenden früheren Bewubtseinsinhalte) zu beachten.

Daß hierdurch die Aufgabe der experimentellen Psychologie in der Tat vollständig umschrieben wird, zeigt ein Überblick über die bisher auf experimentellem Wege in Angriff genommenen psychologischen Untersuchungen.

Sie gliedern sich in die Untersuchung der Intensität und Qualität der Empfindungen, der einfachen Gefühle, der Gehörsvorstellungen, der räumlichen Gesichts - und Tastvorstellungen, der Wahrnehmung bewegter Objekte, der zeitlicben Wahrneh- 
mungen, der Auffassung von kurzdauernden, gleichzeitig oder nacheinander erfolgenden Einwirkungen einer Vielheit von Eindrücken, der sogenannten Reaktionszeiten, der Assoziationen und sogenannter intellektueller Funktionen (der Gedächtnisleistungen, des Lesens, des Schreibens). In allen diesen Fällen handelt es sich aber, wie es nicht anders sein kann, um objektive Zustände und Vorgänge einerseits und ihre Auffassung und Unterscheidung andererseits, wobei das gleichzeitig, unmittelbar zuvor und früber Erlebte in Betracht kommt und berücksichtigt werden muß.

Insbesondere verdient es beachtet zu werden, daß kein prinzipieller Unterschied zwischen der Untersuchung der Intensität und Qualität einfacher Empfindungen in ihrem unmittelbaren Zusammenhange mit den zugrunde liegenden Reizvorgängen und der Feststellung von Gedächtnisleistungen, Reaktionsdauern, oder der als $\mathrm{Ma}$. der Aufmerksamkeit und des Bewußtseinsumfanges in Anspruch genommenen Unterscheidungen gleichzeitiger und successiver Einwirkungen einer Vielheit von Eindrücken besteht. Bei der Darstellung des Gesamtgebietes der Psychölogie pflegen diese Untersuchungen allerdings in verschiedenen Kapiteln behandelt zu werden. Sie bleiben aber. nur bei einer kapitelweisen Betrachtung der Psychologie getrennt, während sie in der Tat einheitlich zusammengehören und eine und dieselbe Methode experimenteller Untersuchung verlangen.

Aus den Beobachtungen, die auf den Zusammenhang objektiven Geschehens und dessen subjektiver Auffassung gerichtet sind, kann aber niemals ein kausaler, auf Äquivalenz gegründeter, sondern bloß ein durch mannigfache konstante und variable Einflüsse bedingter und darum veränderlicber Zusammenhang sich ergeben, so daß sich die Verwertung der Beobachtungsergebnisse auf die in der Untersuchung solcher wechselnden Zusammenhänge und Abhängigkeiten bestehende Wahrscheinlichkeitslehre stützen muß.

Demgemäß ist zunächst anzugeben, was unter Wahrscheinlichkeit zu verstehen und wie sie ihrem Grade nach zu bestimmen ist. 
Zweiter Abschnitt.

\section{Die Wahrscheinlichkeitslehre.}

\section{Gewibheit und Wahrscheinlichkeit.}

Bezeichnet man Unterscheidungen und Verknüpfungen in gleicher Weise als Bestimmungen, so läßt sich jeder Gegenstand als ein System zusammengehöriger Bestimmungen definieren. Er besteht nur auf Grund der Bestimmungen, so daß von ihm nichts übrig bleibt, wenn man alle Bestimmungen wegnimmt.

Die Zusammengehörigkeit der Bestimmungen kann aber gleich den Bestimmungen selbst ursprünglich und schlechthin gegeben sein oder auf bereits vollzogenen Denkakten beruhen. Man hat darum gegebene und abgeleitete Gegenstände oder gegebene und abgeleitete Systeme zusammengehöriger Bestimmungen zu unterscheiden.

Eine Bestimmung, die nicht selbst gegeben ist, kann nur auf Grund gegebener Bestimmungen ausgeführt werden. Denn das Denken existiert nicht unabhängig von den Bestimmungen, in denen es sich tätig erweist. Es kann darum nicht für sich allein bestehen und nun aus sich heraus Bestimmungen erzeugen. Demzufolge müssen stets gegebene Bestimmungen den letzten Ausgangspunkt bilden. Dann findet aber auch jeder abgeleitete Gegenstand in gegebenen Bestimmungen und somit in gegebenen Gegenständen seine Unterlage.

Die gegebenen Gegenstände sind die in räumlicher und zeitlicher Form bestehenden Dinge, die zusammengenommen die Welt ausmachen. Ihnen gehört auch der denkende Mensch selbst an, insofern er eine objektive Existenz besitzt. - Die abgeleiteten Gegenstände hingegen bieten die durch das Denken gewonnene Erkenntnis dar. Sie existieren zunächst im Denken, insofern sie als logisch zusammengehörige Bestimmungen vorhanden sind; es kommt ihnen aber auch ein wirkliches Sein zu, insofern die Be- 


\section{$-18-$}

stimmungen, aus denen sie bestehen, in der objektiv gegebenen Welt Gestalt gewinnen.

Der Bereich der Erfahrungsgegenstände, in denen die Bestimmungen sich verwirklichen, kann der Umfang der Erkenntnis genannt werden. Der Inbegriff der Bestimmungen kann der Inhalt der Erkenntnis heißen. Sagt man „Begriffenes" statt „Erkanntes", so ist der Umfang der Erkenntnis als Begriffsumfang, der Inhalt der Erkenntnis als Begriffsinhalt zu bezeichnen. Es ist aber dann im Auge zu behalten, daß der Begriff kein übersinnliches Sein besitzt, sondern lediglich in einem Systeme zusammengehöriger Bestimmungen besteht und durchaus in der gegebenen Wirklichkeit wurzelt.

Ein solcher Begriff kann ebensowohl durch eine geometrische Figur wie durch eine chemische Substanz, durch einen physikalischen Zustand wie durch eine bestimmte Gemütsverfassung oder Handlungsweise dargestellt werden. Sofern es aber nur auf das Vorhandensein eines bestimmten Inhaltes und eines bestimmten Umfanges ankommt, kann man sich der Einfachheit wegen die mit den fraglichen Bestimmungen behafteten, objektiv existierenden Dinge als Kugeln denken, die in einer Urne beisammen liegen und mit gewissen Zeichen als den Repräsentanten jener Bestimmungen beschrieben sind. Die Mannigfaltigkeit der Kugeln stellt dann den Begriffsumfang, und das System der Zeichen, die jeder Kugel aufgeschrieben sind, den Begriffsinhalt dar.

Soll nun die Erkenntnis, die in einem Systeme von Bestimmungen vorliegt, vermehrt werden, so muß eine neue Bestimmung zu den vorhandenen hinzutreten. Der Begriffsinhalt wird hierdurch jedenfalls größer. Bezüglich des Begriffsumfanges hingegen sind zwei Möglichkeiten zu unterscheiden: entweder gilt die neue Bestimmung in demselben Umfange wie die bereits vorhandenen, oder sie gilt nur teilweise, so daß eine Verringerung des Begriffsumfanges eintritt.

Im ersten Falle sind alle Kugeln der Urne mit dem der neuen Bestimmung entsprechenden Zeichen behaftet. Der Zuwachs an Erkenntnis besteht somit in der Einsicht, daß immer und überall, wo die früher allein beachteten Bestimmungen vorhanden sind, auch die neue Bestimmung sich vorfindet.

Im zweiten Falle haftet das neue Zeichen nicht an jeder Kugel. Es kann an einer einzelnen Kugel ebensowohl vorhandeu 
sein wie auch fehlen. Faßt man aber die Gesamtheit aller Kugeln ins Auge, so sind zwei Gruppen zu unterscheiden: den Kugeln der einen Gruppe kommt das neue Zeichen zu, während es den Kugeln der anderen Gruppe fehlt.

Besteht die erste Gruppe aus $r$ Kugeln, die zweite aus $s$ Kugeln, und ist die Gesamtzahl der Kugeln gleich $t$, so stellt der Quotient

$$
p=\frac{r}{t} \cdot \cdot \cdot \cdot \cdot \cdot \cdot \cdot \cdot \cdot
$$

die relative Häufigkeit für das Auftreten der neuen Bestimmung dar. In entsprechender Weise bezeichnet

$$
q=\frac{s}{t} \cdot \cdot \cdot \cdot \cdot \cdot \cdot \cdot \cdot \cdot
$$

die relative Häufigkeit für das Ausbleiben der neuen Bestimmung. $\mathrm{Da} r+s=t$, so ist

$$
p+q=1 \text {. }
$$

Je nachdem $p$ größer oder kleiner als $q$ ist, wird man das Auftreten oder das Ausbleiben der neuen Bestimmung für wahrscheinlicher halten, und die Wahrscheinlichkeit für das Auftreten oder für das Ausbleiben wird für um so größer angesehen werden, je größer erstenfalls $p$ oder zweitenfalls $q$ ist. Demgemä $\mathrm{b}$ ist unter der Wahrscheinlichkeit oder Unwahrscheinlichkeit einer Bestimmung nichts anderes als die relative Häufigkeit ihres Auftretens oder Ausbleibens zu versteben.

Im Gegensatz hierzu wird eine Bestimmung als gewiß bezeichnet, wenn sie in allen Fällen, die in Frage kommen, eintritt. Sie ist ferner unmöglich, wenn sie niemals eintritt. Es ist alsdann im ersteren Falle $p=1$, $q=0$, und im letzteren Falle $p=0, q=1$.

$\mathrm{Da} \mathfrak{\text { es }}$ zutreffend ist, die Wahrscheinlichkeit einer Bestimmung der relativen Häufigkeit, mit der die Bestimmung auftritt, gleichzusetzen, kann man in allen Fällen, wo von Wahrscheinlichkeit die Rede ist, bestätigt finden.

Sagt man z. B., daß es heute wahrscheinlich regnen werde, so ist dies gleichbedeutend mit der Behauptung, daß in einer großen Anzahl von Fällen bei einer ähnlichen Beschaffenheit der Atmosphäre der Regen häufiger eintreten als ausbleiben wird. 
Nicht anders ist es, wenn man von einem Menschen, der an einer Krankheit darniederliegt, sagt, daß er wahrscheinlich sterben werde. Es liegt auch da die Annahme zugrunde, daß unter ähnlichen, dem vorliegenden Falle vergleichbaren Umständen der Tod häufiger eintreten als ausbleiben werde. Die Annahme kann einer hinreichenden Begründung entbehren. Man spricht alsdann vielleicht nur von einem Gefühle der Wahrscheinlichkeit. Es ist aber auch das Gefühl der Wahrscheinlichkeit nichts anderes als der Zustand des Bewußtseins, der mit einer unsicheren Vermutung über die relative Häufigkeit für das Eintreten des in Frage stehenden Ereignisses verknüpft ist.

Die neve als gewib oder als wahrscheinlich sich darbietende Bestimmung kann auf zwei wesentlich verschiedenen Wegen erkannt werden: auf dem Wege der Deduktion oder auf dem Wege der Induktion.

Geht man von der bereits gewonnenen Erkenntnis aus, die in dem Systeme der vorhandenen Begriffe vorliegt und achtet man auf ihren Zusammenhang, so können zusammenhängende Bestimmungen als zusammenfaßbar sich erweisen, so daß durch die Zusammenfassung eine neue, vorher nicht erkannte Bestimmung sich ergibt. Sie ist als eine aus den bekannten Bestimmungen abgeleitete oder deduzierte Bestimmung und die Ableitung selbst als Deduktion zu bezeichnen.

Eine Deduktion liegt somit vor, wenn man z. B. aus der Gleichheit zweier Seiten eines Dreiecks die Gleichheit der gegenüberliegenden Winkel folgert, oder wenn man auf Grund der gleichmäßigen Beschaffenheit eines Würfels den Schluß zieht, daß bei unbegrenzt wiederholtem Werfen jede Seite gleich häufig erscheinen werde und somit die Wahrscheinlichkeit jeder einzelnen Seite gleich $1 / 6$ sei. In dem einen wie in dem anderen Falle wird aus dem Systeme bekaunter Zeichen, mit denen die Kugeln einer Urne beschrieben sind, das Vorhandensein eines neuen Zeichens erschlossen und als für alle Kugeln oder für einen bestimmten Teil der Kugeln gültig erkannt.

Geht man hingegen von der gegebenen Wirklichkeit aus, auf die in letzter Hinsicht alle Erkenntnis sich gründet, so ist es möglich, daß da, wo die Bestimmungen eines Begriffes Gestalt gewinnen, zugleich eine weitere Bestimmung sich darbietet. Wird dieselbe in das System der vorhandenen Bestimmungen eingefügt, 
so kann sie als induzierte Bestimmung und die Einfügung selbst als Induktion ${ }^{1}$ ) bezeichnet werden.

Eine solche Induktion wurde beispielsweise von Kepler vollzogen, als er zunächst für den Planeten Mars die Annahme einer elliptischen Bewegung bestätigt gefunden hatte und nun den Schluß zog, daß alle Planeten in Ellipsen sich um die Sonne bewegen. In gleicher Weise hat er auch die beiden anderen der drei nach ihm benannten Gesetze der Planetenbewegung induktiv bestimmt. Eine Induktion liegt aber auch vor, wenn ein Arzt in seiner Praxis die Wirkung eines neuen Heilmittels erprobt und feststellt, daß in der Mehrzahl der Fälle eine günstige Wirkung eingetreten und demgemäß auch künftig zu erwarten ist.

War es für Ke pler gewiß, daß alle Planetenbahnen die für den Mars aus einzelnen Beobachtungen berechnete Gestalt haben,

') Es soll nicht unerwähnt bleiben, daß diese Auffassungsweise der Induktion mit der herkömmlichen, die bis auf Aristoteles zuräckgeht, sich nicht völlig deckt. Aristoteles bezeichnet nämlich die Induktion im Gegensatz zum Syllogismus als das Hinaufsteigen vom Einzelnen zum Allgemeinen (Anal. pri. 2, 23; Anal. post. 1, 18 ; Top. 1, 12). Das Einzelne ist aber nicht ein Gegenstand der gegebenen Wirklichkeit, an dem die Bestimmungen eines Begriffs zutage treten, sondern in der Regel ein spezieller Begriff. Die Beispiele des Aristoteles beziehen sich, wie Sigwart (Logik 2, 406) hervorhebt, "meist auf die Spezialbegriffe, und er faßt nicht Einzeltatsachen zu einem untersten Begriffe, sondern spezielle Begriffe zu einem allgemeineren zusammen, bzw. spezielle Regeln zu einer allgemeineren“. Das Allgemeine ist ferner der Begriff in seinem ganzen Umfange, nicht auch ein bloßer Teilumfang des Begriffs, wonach unbeachtet bleibt, daß die Induktion ebensowohl einen für den ganzen Begriffsumfang wie auch eine für einen bloßen Teilumfang gültige Erkenntnis liefern kann. Nun hindert allerdings nichts, das Verallgemeinern als Induktion zu bezeichnen. Dann müßte es aber auch als Induktion gelten, wenn man eine Eigenschaft erst für das spitzwinkelige, dann für das rechtwinkelige und schließlich für das stumpfwinkelige Dreieck beweist und hieraus ihr Vorbandensein für das allgemeine Dreieck erschließt, so daß in der Tat nur eine Zusammenfassung von Deduktionen vorliegt. Andererseits müßte man das Hinzufügen neuer, auf Grund der Erfahrung festgestellter Bestimmungen zu einem vorhandenen Begriffe als ein besonderes Verfahren anerkennen. Es wird aber, wie mir scheint, gerade hierdurch und nicht durch das Merkmal der Verallgemeinerung das Wesen desjenigen Verfahrens gekennzeichnet, das man im Bereich der Erfahrungswissenschaft als Induktion zu bezeichnen gewöhnt ist. 
so ist hingegen für den Arzt die heilende Wirkung der Arznei nur wahrscheinlich; ja es besteht auch dann nur eine allerdings sehr gesteigerte Wahrscheinlichkeit, wenn der Erfolg in allen bisher beobachteten Fällen eingetreten ist.

Der Grund bierfür liegt auf offener Hand: Kepler wußte im voraus, ehe er die Berechnung der Örter für den Planeten Mars unternahm, daß die für den einen Planeten nachgewiesene Bahn von allen Planeten befolgt werde. Es war dies ein unmittelbarer Ausfluß seiner astronomischen Einsicht. Nur die Gestalt der Bahn war unbekannt, nachdem die Kreisbahn als unzutreffend sich erwiesen hatte. - Der Arzt dagegen bat nach unserer Annahme noch keine Erkenntnis über die Wirkung seines Mittels. Er weiß nur, daß der Erfolg entweder eintreten oder ausbleiben wird. Darum kann ihn die Erfahrung nur über die größere oder geringere Häufigkeit des Eintretens oder des Ausbleibens des Erfolges und somit über die Wahrscheinlichkeit oder Unwahrscheinlichkeit desselben belehren.

Deutet man die Planeten in gleicher Weise wie die Patienten des Arztes als Kugeln einer Urne, so genügt demnach im ersteren Falle die Feststellung des neuen Zeichens an einer einzigen Kugel, um es als allen Kugeln anhaftend und somit als gewiß zu erkennen, während im zweiten Falle die Beobachtung der Kugeln nur eine größere oder geringere Wahrscheinlichkeit für das neue Zeichen ergibt. Diese Verschiedenheit ist aber, wie die angeführten Beispiele erkennen lassen, durch die vor der Ausführung der Beobachtungen bereits vorhandene Erkenntnis bedingt.

Demnach gibt es keine allgemein gültige Regel, um den Erfolg einer Induktion in jedem Falle zu bestimmen. Und die Frage Mills ${ }^{1}$ ), „warum in manchen Fällen eine einzige Instanz zu einer vollständigen (d. h. für den ganzen Begriffsumfang gültigen) Induktion ausreiche, wäbrend in anderen Fällen Myriaden übereinstimmender Instanzen, ohne eine einzige bekannte oder vermutete Ausnahme, so sehr wenig dazu beitragen, einen Satz von durchgängiger Allgemeinbeit zu begründen", läßt sich nur dahin beantworten, daß es von der vorhandenen Erkenntnis und den geltenden Grundsätzen wissenschaftlicher Forschung abhängt,

1) John Stuart Mill, System der deduktiven und induktiven Logik (übers. von Gomperz) 1, 367. 
ob eine induktiv erkannte Bestimmung als gewiß oder nur als wahrscheinlich zu gelten hat.

\section{Die Wahrscheinlichkeitsbestimmung.}

Ist Gewißheit vorhanden, so hat die Untersuchung ihren Abschluß gefunden. Hat sich aber nur Wahrscheinlichkeit ergeben, so ist noch ihr Grad zu bestimmen. Denn die Wahrscheinlichkeit wird durch den einen oder den anderen der unendlich vielen, von den Grenzen 0 und 1 eingeschlossenen Zahlenwerte dargestellt, während die Gewißheit, die in dem Zahlenwerte 1 ihren Ausdruck findet, als solche keiner Steigerung und keiner Schwächung fähig ist.

Diese Bestimmung ist ohne weiteres ausführbar, wenn man die in Frage kommenden Häufigkeiten durch einfache und naheliegende Erwägungen feststellen kann. Dies ist bei den Glücksspielen der Fall. Es bezieht sich denn auch die erste, geschichtlich nachweisbare Angabe über Wahrscheinlichkeiten in einem Kommentar zu Dantes göttlicher líomödie vom Jalire 1477 auf die Würfe, die man mit drei Würfeln machen kann ${ }^{1}$ ). Weitergehende Bestimmungen gibt im 16. Jahrhundert Cardano in der Schrift „De ludo aleae" und Galilei (1564 bis 1642) in seinen "Considerazione sopra il giuoco dei dadi“.

Die auf besondere Methoden gegründete mathematische Wahrscheinlichkeitsbestimmung beginnt indessen erst mit den Untersuchungen Pascals (1623 bis 1662) und Fermats (1601 bis 1665). Den äußeren Anlaß bildete die Frage eines Spielers, wie der Einsatz gerechterweise zu verteilen sei, wenn ein Spiel, das auf das Gewinnen einer bestimmten Anzahl von Einzelspielen eingerichtet ist, vor seiner Beendigung abgebrochen wird. Die von beiden Forschern unabhängig voneinander entwickelten Methoden erregten das Interesse der Mathematiker, so daß von da ab die Behandlung von Aufgaben der Wahrscheinlichkeitsrechnung in Aufnahme kam.

Hierbei handelte es sich um deduktive Wahrscheinlichkeitsbestimmungen, die aus der Annahme gleich möglicher Fälle

1) Nach einer Angabe von Libri, Histoire des sciences mathématiques en Italie $2,188$. 
abgeleitet wurden. Es wurden indessen im 17. Jahrhundert auch bereits induktive Wahrscheinlichkeitsbestimmungen ausgeführt.

John Graunt (1620 bis 1674) hat zuerst aus den regelunäßig veröffentlichten Registern der Todesfälle für London Wahrscheinlichkeitsbestimmungen abgeleitet und Schlüsse über die Bevölkerung und das Wachstum dieser Stadt gezogen. Er hat somit erkannt, daß man unbekannte Wahrscheinlichkeitswerte empirisch durch die Beobachtung einer großen Anzahl von Fällen mit hinreichender Genauigkeit bestimmen kann, und solche Bestimmungen selbst ausgeführt. Aus den Geburts- und Todeslisten für Breslau bat ferner Halley (1656 bis 1742) eine Sterblichkeitstafel abgeleitet und daraus für die verschiedenen Lebensalter die Wahrscheinlichkeiten, während einer bestimmten Zeit am Leben $\mathrm{zu}$ bleiben, die Lebenswahrscheinlichkeiten, berechnet.

Es hat sich demnach zu dieser Zeit die Erkenntnis Bahn gebrochen, daß man einen Wahrscheinlichkeitswert für die beliebig große oder auch unendlich große Mannigfaltigkeit von Kugeln einer Urne durch Ziehen einer großen Anzahl einzelner Kugeln bestimmen könne. Dabei wurde als Grundsatz festgehalten, daß die Bestimmung um so zuverlässiger sei, je mehr Kugeln gezogen werden. Da dies durch die Erfahrung seine Bestätigung fand, so konnte eine Untersuchung, ob und wie weit der Grundsatz theoretisch berechtigt sei, als entbehrlich gelten.

Von diesem Standpunkte aus wurden auch in der Folgezeit die sogenannten statistischen Untersuchungen vielfach gepflegt. Insbesondere hat $S \ddot{u} ß \mathrm{mil}$ ch $(1707-1767)$ eine vollständige Lehre von den auf Geburt, Tod und Fortpflanzung beruhenden Gesetzmäßigkeiten innerhalb der menschlichen Gesellschaft entwickelt. Er für seine Person konnte dabei um so mehr eine Begründung des von ihm angewandten Verfahrens entbehren, als er, gestützt auf das Wort der Bibel (1. Mos. 1, 28): „Seid fruchtbar und mehret euch und erfüllet die Erde und machet sie euch untertan ", in Geburt und Tod eine vorbestimmte göttliche Ordnung sah und darum sein Werk „Die göttliche Ordnung in den Veränderungen des menschlichen Geschlechts" nannte. Zur Veranschaulichung benutzte er den Vergleich mit einem vorbeimarschierenden Regiment Soldaten. "Der weiseste Schöpfer und Regierer der Welt", 
so sagt er ${ }^{1}$ ), "lässet das zahlreiche Heer des menschlichen Geschlechts durch die Zeugung aus seinem Nichts hervorgehen, so viel er desselben zum Leben geordnet hat. Der Ewige läßt uns in der Zeit gleichsam vor seinem Angesicht vorbeigehen, bis wir nach Erreichung des einem jeden gesteckten Zieles wiederum von diesem Schauplatze abtreten. Der Auftritt, der Vorübergang vor den Augen des Herrn der Heerscharen und der Abtritt, alles geschieht mit einer bewunderungswürdigen Ordnung. Unser Auftritt im Lande der Lebendigen geschieht allmählich, ohne Gedränge und nach bestimmten Zahlen, die zu dem Heere der Lebendigen wie auch der Wiederabgehenden jederzeit ein regelmäßiges Verhältnis haben. Kurz vor dem Eintritt in das Land der Lebendigen werden noch einige gleichsam ausgemustert. Das sind die Totgeborenen; doch geschieht auch dieses nach gewisser Proportion. Besonders sind bei diesem Hervorgange aus dem Nichts diese beiden Stücke aller Aufmerksamkeit würdig, daß jederzeit gegen 20 Mädchen 21 Söhne kommen; sodann auch, daß die Haufen derer an das Licht kommenden jederzeit etwas größer sind als derer, die wieder in Staub verwandelt werden. Dadurch geschieht es, daß das Heer des menschlichen Geschlechts stets etwas, jedoch auch nach einer steten Proportion, vergrößert wird."

Indessen herrscht auch noch in unseren Tagen die Neigung, die Gewähr für die Sicherheit der Wahrscheinlichkeitsbestimmungen bei statistischen Untersuchungen in der großen Anzahl der Beobachtungen zu suchen, ohne sich um die Berechtigung dieser Annahme und um die Bestimmung der auch bei der größten Beobachtungszahl stets noch übrigbleibenden Unsicherheit zu kümmern.

Um so größer ist das Verdienst Jakob Bernoullis (1654 bis 1705), der die Notwendigkeit, den Einfluß der Anzahl der Beobachtungen auf die Sicherheit der Wahrscheinlichkeitsbestimmung festzustellen, erkannte und untersuchte, ob denn in der Tat durch die Vermehrung der Beobachtungen auch die Sicherheit der Wahrscheinlichkeitsbestimmung immer wächst und nicht vielmehr ein bestimmter Sicherheitsgrad auch bei der größten Beobachtungsanzahl niemals überschritten werden kann. Er kam so zu der Erkenntnis, daß wir den Wahrscheinlichkeitswert nie-

1) Die göttliche Ordnung (2. Aufl.) 1, 52 (1761). 
mals absolut genau, sondern nur mit einer bestimmten Annäherung erhalten, daß aber die Annäherung durch fortgesetzte Beobachtung beliebig weit getrieben werden kann. Ist nämlich der zu bestimmende Wahrscheinlichkeitswert seinem wirklichen Werte nach gleich

$$
p=\frac{r}{t},
$$

so wird es, wie Bernoulli in seiner nachgelassenen, im Jahre 1715 veröffentlichten "Ars conjectandi" im vierten Abschnitte bewiesen hat, mit wachsender Anzahl der Beobachtungen immer wahrscheinlicher, daßder aus den Beobachtungen sich tatsächlich ergebende Wert innerbalb der Grenzen

$$
\frac{r+1}{t} \text { und } \frac{r-1}{t}
$$

als außerhalb derselben liegt, mithin weder größer als $(r+1): t$, noch kleiner als $(r-1): t$ ist. Es kann darum auch, wenn

$$
\frac{n r}{n t} \text { statt } \frac{r}{t}
$$

gesetzt wird, die Anzahl der Beobachtungen so groß gewählt werden, daß mit derselben überwiegenden Wahrscheinlichkeit der beobachtete Wert innerhalb der Grenzen

$$
\frac{n r+1}{n t} \text { und } \frac{n r-1}{n t}
$$

liegt, wo diese Grenzen nun bloß noch um den Betrag

$$
\pm \frac{1}{n t} \text { statt } \pm \frac{1}{t}
$$

vom wahren Werte abweichen.

"Wenn also", so schließt Jakob Bernoulli seine Ausführungen ${ }^{1}$ ), ,alle Ereignisse durch alle Ewigkeit hindurch fortgesetzt beobachtet würden (wodurch schließlich die Wahrscheinlichkeit in volle Gewißheit übergehen müßte), so würde man finden, daß alles in der Welt aus bestimmten Gründen und in bestimmter Gesetzmäßigkeit eintritt, daß wir also gezwungen

1) Ars conjectandi, 4. Teil, Kap. V (Ostwalds Klassiker der exakten Wissenschaften, Nr. 108, S. 107). 
werden, auch bei noch so zufällig erscheinenden Dingen eine gewisse Notwendigkeit und sozusagen ein Fatum anzunehmen."

Dieses Theorem, das in der hergebrachten Auffassungsweise das "Gesetz der großen Zahlen" (wie es von Poisson in den „Recherches sur la probabilité des jugements", 1837, genannt wurde) zum Ausdruck bringt, hat Jakob Bernoulli aus den Gesetzmäßigkeiten der binomischen Reihe abgeleitet. Die in den Lehrbüchern der Wahrscheinlichkeitsrechnung übliche Darstellungsweise beruht indessen auf einer an die Stirlingsche Formel anknüpfenden Umformung der Glieder der binomischen Reihe für den Fall einer großen Anzahl von Reihengliedern, die zu dem gewöhnlichen, auch in der Fehlertheorie durch Gauss in Aufnahme gekommenen Exponentialgesetze geführt hat.

Einen einfacheren Beweis, der zugleich die Voraussetzungen, an welche die induktive Wahrscheinlichkeitsbestimmung gebunden ist, deutlich hervortreten läßt, liefern folgende Überlegungen.

Sind in einer Urne ohne unser Wissen unter $t$ Kugeln $r$ weiße und $s$ schwarze Kugeln, so daß $t=r+s$ ist, und die relative Häufigkeit oder die Wahrscheinlichkeit einer weißen Kugel und einer schwarzen Kugel durch

$$
p=\frac{r}{t} \text { und } q=\frac{s}{t} \quad . \quad . \quad . \quad .
$$

angegeben wird, so kann man beim Ziehen einer einzelnen Kugel jede der $t$ Kugeln, also jede der $r$ weißen und jede der $s$ schwarzen Kugeln treffen. Es gibt somit

$$
t=r+s \text {. }
$$

Möglichkeiten. Zieht man hingegen zwei Kugeln nacheinander, wobei die zuerst gezogene Kugel wieder zurückgelegt wird, ehe man die zweite zieht, so bestehen

$$
t^{2}=r^{2}+2 r s+s^{2} \text {. }
$$

Möglichkeiten, indem man $r^{2}$ mal zwei weiße Kugeln, $2 r s$ mal zuerst eine weiße und dann eine schwarze oder zuerst eine schwarze und dann eine weiße Kugel, und $s^{2}$ mal zwei schwarze Kugeln treffen kann. In entsprechender Weise ergeben sich, wenn $m$ Kugeln nacheinander unter jedesmaligem Zurücklegen der jeweils gezogenen Kugeln gezogen werden,

$t^{m}=r^{m}+\frac{m}{1} r^{m-1} s+\frac{m(m-1)}{1.2} r^{m-2} s^{2}+\cdots+s^{m}$ 
Möglichkeiten, so daß in

$$
\frac{m(m-1) \ldots(m-i+1)}{1.2 \ldots i} r^{m-i} s^{i}
$$

Fällen $m-i$ weiße und $i$ schwarze Kugeln in beliebiger Reihenfolge (für $i=0,1,2 \ldots m$ ) sich ergeben.

Von diesen Möglichkeiten kann sich stets nur eine einzige unter Ausschluß aller übrigen verwirklichen. Die Anzahl der weißen Kugeln kann daher gleich 0,1,2 $\ldots m-1, m$ und zugleich diejenige der schwarzen Kugeln gleich $m, m-1, m-2 \ldots 1,0$ sein, so daß zunächst gar nicht einzusehen ist, mit welchem Rechte aus der gezogenen Anzahl auf die wirklich in der Urne vorhandene unbekannte Anzahl geschlossen wird.

In der Tat bedarf es zweier Voraussetzungen, wenn überhaupt eine induktive Wabrscheinlichkeitsbestimmung möglich sein soll: $\operatorname{man} m u B$ voraussetzen, daß erstens bei einer unbegrenzten Wiederholung der ausgeführten $m$ Züge jede der $t^{m}$ Möglichkeiten in gleicher Häufigkeit zur Verwirklichung gelangt, und daß zweitens das aus allen diesen unendlich vielen Fällen sich ergebende arithmetische Mittel der weißen und der schwarzen Kugeln mit den aus den wirklich ausgeführten $m$ Zügen gefundenen Anzahlen der weißen und schwarzen Kugeln übereinstimmt.

Der ersten Voraussetzung gemäß hat die unbegrenzte Wiederholung von $m$ Zügen das Auftreten von

$$
0, \quad 1, \quad 2, \quad \ldots m
$$

weißen und zugleich von

$$
m, \quad m-1, \quad m-2, \quad \ldots 0
$$

schwarzen Kugeln zur Folge, wobei jede Anzahl sich unter $t^{m}$ Fällen durchschnittlich in der Häufigkeit

$$
s^{m}, \quad \frac{m}{1} r s^{m-1}, \quad \frac{m(m-1)}{1.2} r^{2} s^{m-2}, \ldots r^{m}
$$

oder, wenn $p=r: t$ und $q=s: t$ gesetzt wird, in der relativen Häufigkeit

$$
q^{m}, \quad \frac{m}{1} p q^{m-1}, \quad \frac{m(m-1)}{1.2} p^{2} q^{m-2}, \ldots p^{m}
$$


ergibt. Mit der nämlichen Häufigkeit findet man demnach für die weißen Kugeln die Verhältnis- oder Wahrscheinlichkeitswerte

$$
0, \quad \frac{1}{m}, \quad \frac{2}{m}, \quad \ldots 1
$$

und zugleich für die schwarzen Kugeln die Verhältnis- oder Wahrscheinlichkeitswerte

$$
1, \quad \frac{m-1}{m}, \quad \frac{m-2}{m}, \quad \ldots 0 .
$$

Alle diese Wahrscheinlichkeitswerte sind unter Bezugnahme auf die angegebenen Häufigkeiten ihres Auftretens zu berücksichtigen. Es kann folglich der aus den $m$ tatsächlich ausgeführten Zügen berechnete Wahrscheinlichkeitswert der weißen oder schwarzen Kugeln nicht für sich allein, sondern lediglich als Repräsentant der ganzen Mannigfaltigkeit von Wahrscheinlichkeitswerten, die bei unbegrenzter Wiederbolung der $m$ Züge auftreten würden, Geltung gewinnen.

Diese Mannigfaltigkeit bleibt aber unbekannt, wenn man bloß annimmt, daß irgend ein Glied derselben mit dem beobachteten Wahrscheinlichkeitswerte übereinstimmt. Es gilt daher eine Vereinbarung zu treffen, welches Glied dem beobachteten Werte gleichgesetzt werden soll. Da man das am häufigsten vorkommende Glied wählen wird, und dasselbe mit dem arithmetischen Mittel aller der Mannigfaltigkeit angehörenden Werte völlig oder nahezu übereinstimmt, so ist im Einklang mit der zweiten Voraussetzung das arithmetische Mittel dem beobachteten Werte gleich zu setzen.

Es ist nun das Mittel aus der Anzahl der weißen Kugeln gleich $0 \cdot q^{m}+1 \cdot \frac{m}{1} p q^{m-1}+2 \cdot \frac{m(m-1)}{1.2} p^{2} q^{m-2}+\cdots$

$$
+m \cdot p^{m}=m p \text {. }
$$

und ebenso das Mittel aus der Anzahl der schwarzen Kugeln gleich $m \cdot q^{m}+(m-1) \cdot \frac{m}{1} p q^{m-1}+(m-2) \cdot \frac{m(m-1)}{1.2} p^{2} q^{m-2}+\ldots$
$+0 \cdot p^{m}=m q \cdot$. . . . . . $(9)$ so daß $p$ und $q$ die arithmetischen Mittel für die Wahrscheinlichkeitswerte der weißen und schwarzen Urnenkugeln sind. Es sind 
daher die unbekannten Wahrscheinlichkeitswerte $p$ und $q$ der weißen und schwarzen Urnenkugeln den aus den $m$ Zügen bestimmten Wahrscheinlichkeitswerten gleichzusetzen.

Die beobachteten Wahrscheinlichkeitswerte $p$ und $q$ der weißen und schwarzen Kugeln repräsentieren demnach die Mannigfaltigkeiten der Werte

und

$$
0, \quad \frac{1}{m}, \quad \frac{2}{m}, \cdots \frac{m-1}{m}, \quad 1
$$

$$
1, \frac{m-1}{m}, \frac{m-2}{m}, \cdots \frac{1}{m}, \quad 0
$$

in den zugehörigen relativen Häufigkeiten:

$$
q^{m}, \quad \frac{m}{1} p q^{m-1}, \quad \frac{m(m-1)}{1.2} p^{2} q^{m-2}, \cdots \frac{m}{1} p^{m-1} q, p^{m} .
$$

Es kann aber in vielen Fällen als ausreichend gelten, eine Mannigfaltigkeit reeller positiver Werte $a_{1}, a_{2}, a_{3}, \ldots a_{n}$ durch das arithmetische Mittel

$$
b=\frac{1}{n}\left(a_{1}+a_{2}+a_{3}+\cdots+a_{n}\right) . .
$$

und durch das Mittel der quadratischen Abweichungen

$$
\varepsilon_{2}^{2}=\frac{1}{n}\left\{\left(a_{1}-b\right)^{2}+\left(a_{2}-b\right)^{2}+\cdots+\left(a_{n}-b\right)^{2}\right\}
$$

ihrer Lage und Ausdehnung nach zu bestimmen und symbolisch durch

$$
b \pm \varepsilon_{2} \cdot \text {. . . . . . . }
$$

darzustellen. Dementsprechend lassen sich auch die Mannigfaltigkeiten der Wahrscheinlichkeitswerte für die weißen und schwarzen Kugeln durch die arithmetischen Mittelwerte $p$ und $q$ im Verein mit dem, beiden gemeinsam zugehörigen Mittel der Abweichungsquadrate:

$$
\left.\begin{array}{c}
(0-p)^{2} \cdot q^{m}+\left(\frac{1}{m}-p\right)^{2} \cdot \frac{m}{1} p q^{m-1}+\cdots+(1-p)^{2} \cdot p^{m}= \\
(1-q)^{2} \cdot q^{m}+\left(\frac{m-1}{m}-q\right)^{2} \cdot \frac{m}{1} p q^{m-1}+\cdots+(0-q)^{2} \cdot p^{m} \\
=\frac{1}{m} p q
\end{array}\right\}
$$


in der Form

darstellen.

$$
\left.\begin{array}{l}
p \pm \sqrt{\frac{1}{m} p q} \\
q \pm \sqrt{\frac{1}{m} p q}
\end{array}\right\}
$$

Nachdem so die Bedeutung der induktiven Wahrscheinlichkeitsbestimmung vollkommen klar gestellt ist, erledigt sich der Beweis des Bernoullischen Theorems durch den Hinweis darauf, daß mit wachsendem $m$ die mittlere quadratische Abweichung immer kleiner wird und somit die ganze Mannigfaltigkeit der Wahrscheinlichkeitswerte sich immer dichter um den als arithmetisches Mittel gedeuteten, empirisch bestimmten Wahrscheinlichkeitswert schart.

Hat man beispielsweise unter $m=10,100,1000$ Zügen gleich viel weibe und schwarze Kugeln gefunden, so repräsentieren die hieraus sich ergebenden Wahrscheinlichkeitswerte der weißen und schwarzen Kugeln die Mannigfaltigkeiten

$$
0,5 \pm 0,158, \quad 0,5 \pm 0,050, \quad 0,5 \pm 0,016,
$$

woraus die stets geringer werdende Streuung der Mannigfaltigkeitswerte ersichtlich wird.

Um schließlich noch zu zeigen, inwieweit die der induktiven Wahrscheinlichkeitsbestimmung zugrunde liegenden Voraussetzungen von der Erfahrung bestätigt werden, gebe ich in der Tabelle I (siehe folgende Seite) die empirischen Häufigkeiten $z$ an, wie oft bei $n$ maliger Ausführung eines Zuges von je $m$ Kugeln sich $a$ weiße Kugeln ergeben haben ${ }^{1}$ ). Zum Vergleich wurden die voraussetzungsgemäß zu erwartenden theoretischen Anzahlen $z$ beigefügt.

Die 4800 Züge ergeben zusammengenommen 2422 weiße Kugeln, so daß die empirisch bestimmte Wahrscheinlichkeit der weißen Kugeln die Mannigfaltigkeit

repräsentiert.

$$
0,5046 \pm 0,0072
$$

1) An Stelle der traditionellen Kugeln dienten Karten, von welchen nach erfolgter Mischung je eine gezogen und, nachdem ihre Marke notiert war, wieder zu den übrigen gesteckt wurde. Aus 4800 einzelnen Zügen wurden sodann die $n$ Gruppen von je $m$ Zügen gebildet. 
Tabelle I.

\begin{tabular}{|c|c|c|c|c|c|c|c|c|}
\hline \multicolumn{3}{|c|}{$m=10, n=480$} & \multicolumn{3}{|c|}{$m=20, n=240$} & \multicolumn{3}{|c|}{$m=40, n=120$} \\
\hline \multirow{2}{*}{$a$} & \multicolumn{2}{|c|}{$z$} & \multirow{2}{*}{$a$} & \multicolumn{2}{|c|}{$z$} & \multirow{2}{*}{$a$} & \multicolumn{2}{|c|}{$z$} \\
\hline & emp. & theor. & & emp. & theor. & & emp. & theor. \\
\hline 0 & 1 & 0,5 & 4 & 2 & 1 & 12 & 1 & 1 \\
\hline 1 & 3 & 5 & 5 & 3 & 3,5 & 13 & 3 & 1 \\
\hline 2 & 24 & 21 & 6 & 9 & 9 & 14 & 5 & 2 \\
\hline 3 & 63 & 56 & 7 & 15 & 18 & 15 & 4 & 5 \\
\hline 4 & 86 & 98,5 & 8 & 35 & 29 & 16 & 6 & 7 \\
\hline 5 & 109 & 118 & 9 & 41 & 38.5 & 17 & 12 & 10 \\
\hline 6 & 106 & 98,5 & 10 & 31 & 42 & 18 & 12 & 12,5 \\
\hline 7 & 56 & 56 & 11 & 36 & 38,5 & 19 & 6 & 14 \\
\hline 8 & 24 & 21 & 12 & 28 & 29 & 20 & 9 & 15 \\
\hline 9 & 7 & 5 & 13 & 25 & 18 & 21 & 19 & 14 \\
\hline \multirow[t]{8}{*}{10} & 1 & 0,5 & 14 & 4 & 9 & 22 & 11 & 12,5 \\
\hline & \multirow[t]{7}{*}{480} & \multirow[t]{7}{*}{480} & 15 & 8 & 3,5 & 23 & 12 & 10 \\
\hline & & & \multirow[t]{6}{*}{16} & 3 & 1 & 24 & 5 & 7 \\
\hline & & & & \multirow[t]{5}{*}{240} & \multirow[t]{5}{*}{240} & 25 & 5 & 5 \\
\hline & & & & & & 26 & 4 & 2 \\
\hline & & & & & & 27 & 3 & 1 \\
\hline & & & & & & 28 & 3 & 1 \\
\hline & & & & & & & 120 & 120 \\
\hline
\end{tabular}

Die hier zwischen den theoretischen und empirischen Werten auftretenden Differenzen beweisen nicht die Unzulässigkeit der zugrunde gelegten Voraussetzungen, da erst für unendlich großes $n$ eine vollkommene Übereinstimmung gefordert werden darf.

Auf Grund der gewonnenen Einsicht in das Wesen der Wahrscheinlichkeit und ihre Bestimmungsweise könnten nunmehr die auf die Verwendung von Maß und Zahl gegründeten Methoden der experimentellen Psychologie - die psychischen Maßmethoden ohne weiteres systematisch entwickelt werden.

Um jedoch auch ein Verständnis für die geschichtliche Entwickelung dieser Methoden und den hierdurch bedingten Grad ihrer gegenwärtigen Ausbildung zu ermöglichen, soll zunächst 
gezeigt werden, wie die exakte naturwissenschaftliche Forschung zu der Berücksichtigung subjektiver Faktoren veranlaßt wurde, und wie aus diesen Anfängen die heute im Gebiete der experimentellen Psychologie verwendeten Maßmethoden sich entwickelt haben.

\section{Dritter Abschnitt.}

\section{Die Maßbestimmungen bei der Berïicksichtigung subjektiver Faktoren im Bereiche der natur- wissenschaftlichen Forsehung.}

\section{Die Beobachtungsfehler.}

Wer durch die Beobachtung der gegebenen Wirklichkeit eine Erkenntnis zu gewinnen sucht, kann sich der Tatsache nicht verschließen, daß er bei der Ausführung der Beobachtung und bei der Verwertung der Beobachtungsergebnisse Irrtümern ausgesetzt ist.

Mit Rücksicht hierauf weist schon Francis Bacon, der in der Interpretation der Natur das Ziel aller Erkenntnis sieht, in seinem "Novum Organon" (1620) auf die Trugbilder oder Idole hin, die den Geist zur unbefangenen Naturerkenntnis unfähig machen. Er unterscheidet vier Arten von Trugbildern. Die Idola tribus sind in der menschlichen Natur begründet und beeinflussen darum jeden Menschen. Zu ihnen gehört insbesondere die Neigung, sich von Gefühlen und Stimmungen beherrschen zu lassen. Die Idola specus rühren von der individuellen Beschaffenheit des Menschen her, der zufolge jeder durch seine Brille oder - wie Bacon sagt - aus seiner eigenen Höhle heraus die Welt betrachtet. Sie bestehen in besonderen, durch natürliche Veranlagung und den Bildungsgang bedingten Neigungen. Die Idola fori und die Idola theatri schließlich veranlassen Vorurteile, die im Verkehr der Menschen untereinander und in herrschenden, 
zur Mode gewordenen Theorien und philosophischen Anschauungen ihre Quelle haben.

Aber auch die Sinne haben - was Bacon nicht unerwähnt läßt - ihre Mängel: sie versagen und geben gar keine Auskunft über die Dinge oder sie täuschen uns.

Demgemäß machen sich insbesondere bei der Ausführung von Beobachtungen zwei Klassen subjektiver Faktoren geltend. Sie werden nach der Ausdrucksweise Bacons einesteils durch die Trugbilder des Verstandes, anderenteils durch die Mängel der Sinne veranlaßt. Sie sollen jedoch hier einesteils als in der Sinneswabrnehmung liegend und anderenteils als in der Geistesverfassung oder in der Persönlichkeit des Beobachters überhaupt begründet unterschieden werden.

Man kann hoffen, dem schädlichen Einflusse dieser Faktoren zu entgehen, wenn man die Beobachtungen mit möglichst großer Sorgfalt unter den erreichbar günstigsten Umständen anstellt. Dies wurde offenbar während der ersten Blütezeit der exakten naturwissenschaftlichen Forschung im Verlaufe des 17. Jahrhunderts als Grundsatz festgehalten, so daß eine Berücksichtigung der Beobachtungsfehler nicht nötig schien.

So ist denn Roger Cotes der erste, der in einer nachgelassenen, im Jahre 1722 veröffentlichten Abhandlung die Fehler von Beobachtungsgrößen, die voneinander abhängig und durch Gleichungen miteinander verknüpft sind, untersucht und die Beobachtungsfehler bei der Bestimmung eines Punktes mit Gewichten von reziproker Größe vergleicht, die den beobachteten Punkten anzuhängen seien, damit man in ihrem Schwerpunkte den wahren Punkt erhalte.

Daß es aber vorteilhafter sei, statt nur einzelne Beobachtungen möglichst sorgfältig auszuführen, die Beobachtungen zu häufen und zu Mittelwerten zu vereinigen, hebt erst Thomas Simpson in einer Abhandlung vom Jahre 1757 hervor. Er ist sich auch darüber klar, daß die mathematische Behandlung der beobachteten Größen eine Voraussetzung über die Häufigkeit der Beobachtungsfehler erfordert und zeigt an einem Beispiele, wie mit zunehmender Anzahl der Beobachtungen die Zuverlässigkeit des arithmetischen Mittels wächst.

Weitergehende und ausführlichere Erörterungen stellt zu derselben Zeit Lambert gelegentlich seiner grundlegenden, im 
Jahre 1760 veröffentlichten photometrischen Untersuchungen an. Er findet, daß bei gleicher Wahrscheinlichkeit der positiven und der negativen Fehler das arithmetische Mittel der wahrscheinlichste Wert sei, der bei unbegrenzter Wiederholung der Beobachtung vom wahren Werte sich nicht unterscheide. Daß aber auch bei einer endlichen Anzahl von Beobachtungen die Sicherheit des arithmetischen Mittels mit der Häufung der Beobachtungen wachse, scheint ihm durch das Bernoullische Theorem sichergestellt, wofern nur die Annahme richtig ist, daß größere Fehler seltener auftreten als kleinere. Darum untersucht er die Fehlerquellen und gelangt dazu, drei Fehlerarten zu unterscheiden. Die Fehler der ersten Art werden, da es sich um photometrische Untersuchungen handelt, durch die unvermeidliche Unbestimmtheit im Urteil des Auges veranlaßt. Diejenigen der zweiten Art sind in der Unachtsamkeit des Beobachters begründet, der niemals gleichmäßig wachen könne, auch wenn er noch mehr Augen als Argus selbst hätte. Die Fehler der dritten Art endlich rühren von der Beschaffenheit der Instrumente und anderen, dem Einfluß des Beobachters entzogenen Umständen her.

Von diesen letzteren sieht Lambert ab. Bezüglich der Fehler der ersten und der zweiten Art hingegen weist er nach, daß sie um so seltener auftreten, je größer sie sind. Demnach habe das arithmetische Mittel in der Tat als der wahrscheinlichste Wert zu gelten. Um jedoch auch ein Urteil über die Sicherheit dieser Bestimmung zu gewinnen, berechnet Lambert nach Ausschluß der Beobachtungen, die mit dem größten Fehler behaftet sind, von neuem das Mittel. Die Differenz beider Mittel gilt ihm als Maß der Unsicherheit für das aus allen Beobachtungen gefundene Mittel.

Auf Grund dieser Annahme kommt Lambert zu einer "Theorie der Zuverlässigkeit der Beobachtungen und Versuche", in der er die zunächst für die Messungen einer und derselben Größe aufgestellten Grundsätze auf den Fall, wo eine gesetzmäßige Folge von Größen zu bestimmen ist, überträgt und an Beispielen erläutert. Auch betrachtet er in den "Bemerkungen und Zusätzen zur praktischen Geometrie" bei der Untersuchung des Mittels zwischen den Fehlern besondere, den Umständen angepaßte gesetzmäßige Verteilungen der Fehler. Beide Abhand- 
lungen findet man in den „Beiträgen zum Gebrauch der Matbematik und deren Anwendung" vom Jahre 1765.

Von dem Nutzen der Methode, das Mittel aus den Ergebnissen mehrerer Beobachtungen zu nehmen, und von der Prüfung derselben mittels der Wahrscheinlichkeitsrechnung handelt ferner Lagrange im fünften Bande der Miscellanea Taurinensia (1770 bis 1773). Er setzt dabei Fehler von bestimmter Größe in bestimmter Häufigkeit voraus und bestimmt Wahrscheinlichkeiten, die sich auf das Mittel aus einer Anzahl von Beobachtungen beziehen.

Eine von Lagrange und Lambert gelegentlich betrachtete Fehlerverteilung setzt auch Daniel Bernoulli in einer Abhand-

Fig. 1 .

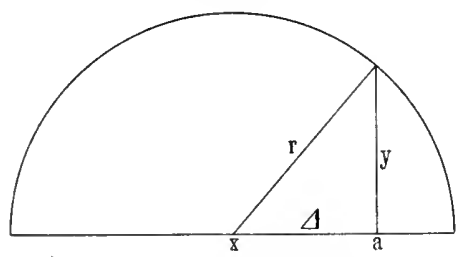

lung der Petersburger Akademie über die Ausgleichung der Beobachtungsfehler (1778) voraus, um den wahrscheinlichsten Wert aus einer Reihe beobachteter Werte abzuleiten. Es wird dort die Häufigkeit des Fehlers von der Größe $\Delta$ des Fehlers von der
rve bestimmt, so daß

$$
y=\sqrt{r^{2}-\Delta^{2}} \text {. }
$$

wenn $r$ den Kreisradius bedeutet.

Sind nun die Werte $a, b, c \ldots$ beobachtet worden und wird der wahrscheinlichste Wert durch $x$ bezeichnet, so bieten sich in den Differenzen $a-x, b-x, c-x, \ldots$ die Beobachtungsfehler dar, und es hat nun derjenige Wert $x$ als der wahrscheinlichste zu gelten, für den das Produkt

$$
\sqrt{r^{2}-(x-a)^{2}} \cdot \sqrt{r^{2}-(x-b)^{2}} \cdot \sqrt{r^{2}-(x-c)^{2}} \ldots
$$

ein Maximum wird.

Es fehlt somit bloß noch die Aufstellung eines einwandfreien Fehlergesetzes, um eine vollkommene Lösung des Problems, wiederholte Beobachtungen einer und derselben Größe auszugleichen, zu erhalten.

Das von Daniel Bernoulli angenommene Gesetz ist nämlich nicht nur der Form nach ungeeignet, insofern es zu einem unbequemen Rechenverfahren führt, sondern auch prinzipiellen 
Bedenken unterworfen, weil es einen und denselben endlichen Bereich möglicher Fehler für alle Beobachtungsreihen voraussetzt. Denn der Inhalt des Halbkreises, der den Inbegriff aller möglichen Fehler vor Augen stellt, ist gleich 1, und somit $1 / 2 r^{2} \pi=1$ oder $r=\sqrt{2: \pi}$ zu setzen, so daß sich alle Fehler stets auf den durch diesen Wert abgegrenzten Bereich in der durch die Kreiskurve angegebenen Weise verteilen müßten. Eine Bestimmung der Zuverlässigkeit der Beobachtungen auf Grund einer bald enger sich zusammenschließenden, bald weiter sich ausdehnenden Fehlerverteilung wäre demgemäß ausgeschlossen; es müßte vielmehr von Haus aus allen Beobachtungsreihen die gleiche Genauigkeit zukommen, da sich jede mit der nämlichen Regelmäßigkeit über ein und dasselbe Intervall erstrecken würde. Und wenn auch eine gewisse Veränderung der Fehlerverteilung leicht dadurch sich erreichen ließe, daß der Halbkreis durch ein Kreissegment vom Inhalte 1 ersetzt würde, so bliebe doch die Abhängigkeit der Fehlerverteilung von dem Fehlermaximum bestehen, das sich auf Grund der Beobachtungen nicht mit Sicherheit feststellen läßt, sondern erfahrungsgemäß großen Schwankungen unterliegt. Nicht anders wäre es, wenn das Segment einer Ellipse oder einer Parabel an Stelle des Kreissegmentes gewählt würde.

Eine von diesen Mängeln freie Fehlertheorie haben erst Gauss und Laplace entwickelt.

In der "Theoria motus corporum coelestium" (1809) zeigt Gauss zunächst, wie die wahrscheinlichsten Werte beobachteter Größen auf Grund eines die Fehlerverteilung bestimmenden Gesetzes abzuleiten sind. Um aber zu einem hinreichend begründeten Fehlergesetze zu gelangen, geht er von der Hypothese aus, da $\beta$ bei der wiederholten unmittelbaren Beobachtung einer Größe das arithmetische Mittel zwischen allen beobachteten Werten der wahrscheinlichste Wert sei. Auf Grund dieser Annahme findet er die Wahrscheinlichkeit $y$ eines Fehlers von der Größe $\Delta$ bestimmt durch ${ }^{1}$ )

$$
y=\frac{h}{\sqrt{\pi}} \exp \left(-h^{2} \Delta^{2}\right) \text {. . . . . }
$$

$\left.{ }^{1}\right)$ Es wird hier die unbequeme Bezeichnungsweise $e^{x}$ durch die bequemere $\exp (x)$ ersetzt. 
Es ist daher, wenn man die beobachteten Werte durch $a, b, c \ldots$ und den wahrscheinlichsten Wert durch $x$ bezeichnet, das Produkt

$$
\begin{gathered}
\frac{h}{\sqrt{\pi}} \exp \left\{-h^{2}(a-x)^{2}\right\} \cdot \frac{h}{\sqrt{\pi}} \exp \left\{-h^{2}(b-x)^{2}\right\} \\
\cdot \frac{h}{\sqrt{\pi}} \exp \left\{-h^{2}(c-x)^{2}\right\} \ldots
\end{gathered}
$$

zu einem Maximum und somit - wie unmittelbar erhellt - die Summe

$$
(a-x)^{2}+(b-x)^{2}+(c-x)^{2}+\cdots
$$

zu einem Minimum zu machen, woraus sich der Grundsatz orgibt, daß auch bei der Berechnung mehrerer Unbekannten dasjenige Wertensystem als das wahrscheinlichste zu betrachten ist, für welches die Quadrate der Abweichungen zwischen den beobachteten und den berechneten Werten ein Minimum wird. Zugleich bietet sich in dem Parameter $h$ des Fehlergesetzes das Maß der Genauigkeit für die Beobachtungen dar, indem mit wachsendem $h$ die Wahrscheinlichkeit der größeren Fehler geringer und diejenige der kleineren Fehler stärker wird, wie dies aus dem Vergleich der ausgezogenen Kurve mit der punktierten, einem 1,5 mal größeren $h$ zugehörigen Kurve in Fig. 2 erhellt.

Fig. 2.

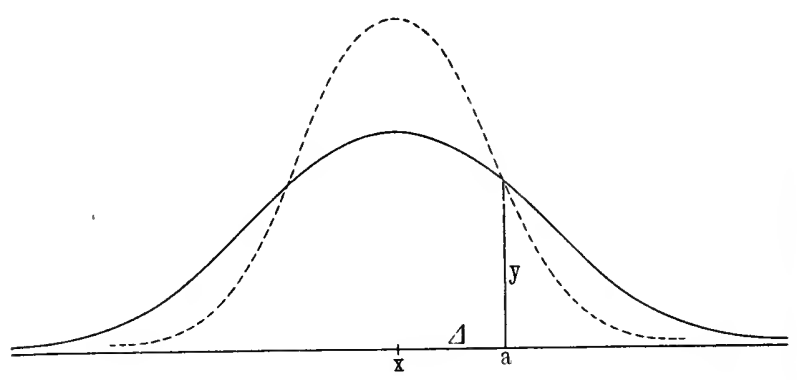

Auf diesem Wege gelangte Gauss zu der von ihm seit dem Jahre 1795 benutzten "Methode der kleinsten Quadrate", die auch von Legendre in den "Nouvelles méthodes pour la détermination des orbites des comètes" (1806) entwickelt wurde. 
Laplace bewies sodann in der "Théorie analytique des Probabilités" (1812) auf einem ganz anderen Wege, daß diese Behandlung der Beobachtungsergebnisse in jedem Falle, ganz unabhängig von der Annahme eines Fehlergesetzes, den Vorzug verdient, falls nur die Anzahl der Beobachtungen sehr groß ist.

Gauss hingegen zeigte bei der abermaligen Behandlung des Problems in der "Theoria combinationis observationum erroribus minimis obnoxiae" (1821), daß die Methode der kleinsten Quadrate für jedwedes Fehlergesetz und jegliche Anzahl von Beobachtungen zur schlechthin besten Kombination der Beobachtungen führt, wenn man den mittleren Fehler einer von konstanten Fehlern freien Beobachtungsreihe nicht wie Laplace durch das Mittel der einfachen, dem absoluten Betrage nach genommenen Fehler, sondern durch das Mittel der Fehlerquadrate definiert. Dann ist der in dieser Weise bestimmte mittlere Fehler als $\mathrm{Ma}$ für die Unsicherheit der Beobachtungen anzusehen, so daß von zwei Beobachtungsreiben diejenige für die genauere zu halten ist, die mit dem kleineren mittleren Fehler behaftet ist.

Hiernach findet das von Lambert zuerst in Angriff genommene Problem, aus den beobachteten Größen ein $\mathrm{Mab}$ für die Sicherheit der Beobachtungen zu gewinnen, durch Gauss und Laplace seine vollkommene Lösung, indem einesteils der Parameter $h$ des Fehlergesetzes als $\mathrm{MaL}$ der Sicherheit und anderenteils, ohne Rücksicht auf ein Fehlergesetz, der mittlere Fehler als $\mathrm{Maß}$ der Unsicherheit dient.

Die Sicherheit oder Unsicherheit der Beobachtungen ist aber, wie namentlich Lambert ausfübrlich hervorgehoben hat, wesentlich von dem Einfluß subjektiver Faktoren abhängig. Insoweit dies der Fall ist, hat demgemäß sowohl der Parameter $h$ des Fehlergesetzes als auch der ohne Rücksicht auf ein etwa geltendes Fehlergesetz berechnete mittlere Fehler einer Beobachtungsreihe als ein den Einfluß subjektiver Faktoren bestimmender Maßwert zu gelten.

\section{Die Ungenauigkeit der Sinneswahrnehmung und die sonstigen subjektiven Faktoren.}

Die verschiedenartigen subjektiven Faktoren bedürfen im Interesse der Naturbeobachtung keiner ins einzelne gehenden 
Unterscheidung und Untersuchung, so lange dafür gesorgt werden kann, daß sie innerhalb einer Gruppe zusammengehöriger Beobachtungsreiben mit hinreichender Konstanz zur Geltung kommeu. Dies trifft beispielsweise zu, wenn es sich um das Messen großer und kleiner Winkel, hoher und niederer Barometerstände, oder um sonstige durch Ablesungen an einer Skala von gleichmäßiger Beschaffenheit auszuführende Messungen handelt.

Anders ist es, wenn es sich um das unmittelbare Erfassen und Beurteilen verschieden starker Reizgrößen handelt. Denn es macht sich alsdann die Ungenauigkeit der Sinneswahrnehmung in verschiedenem Maße geltend, so daß man wissen muß, mit welchem Betrage sie in dem einen und in dem anderen Falle in Rechnung zu stellen ist.

Demgemäß mußte bei der Begründung und dem Ausbau der Photometrie das Bedürfnis sich geltend machen, die Fähigkeit des Auges zur Helligkeitsunterscheidung festzustellen. Es gibt nämlich, wie Lambert ${ }^{1}$ ) in seiner Photometrie hervorhebt, kein Instrument, mit welchem man die Intensität des Lichtes, ähnlich wie mit der Wage das Gewicht oder mit dem Thermometer die Wärme, messen könnte. Wir haben kein Photometer von solcher Art, daß es unter der unmitttelbaren Einwirkung des Lichtes die Intensität desselben anzeigen würde. Wir sind vielmehr auf unser Auge angewiesen. Darum forscht Lambert nach den Gründen, die das Urteil des Auges unsicher machen. Er findet sie in der Änderung der Pupillenöffnung unter dem Einflusse des in das Auge fallenden Lichtes und in der Fähigkeit des Auges, sich verschiedenen Helligkeitsstufen anzupassen. Dazu kommt noch die allen Sinnesgebieten gemeinsame Eigentümlichkeit, daß die stärkere Empfindung die schwächere unterdrückt. "So scheint eine Kerze im Sonnenschein gar keine Helligkeit zu besitzen; dagegen vermag sie das Licht, welches nachts von faulendem Holze verbreitet wird, so unsichtbar zu machen, als wenn es gar nicht vorhanden wäre." Soll trotz dieser Mängel eine Photometrie möglich sein, so muß es, wie Lambert hervorhebt, als Grundsatz anerkannt werden, daß eine Erscheinung dieselbe sei, so oft dasselbe Auge auf dieselbe Weise affiziert werde, und daß mithin

1) Lambert, Photometrie, deutsch von Anding, Ostwalds Klassiker der exakten Wissenschaften, Nr. $31, \S 6$ bis 30 . 
eine Verschiedenheit in der Helligkeitswahrnehmung entweder durch eine veränderte Beschaffenheit oder durch eine veränderte Reizung des Auges bedingt sei. Man kann sich nun zwar überzeugen, daß in der Tat "Gegenstände, welche offenbar heller sind, durch das Urteil des Auges auch heller gefunden werden ". Es ist aber nicht möglich anzugeben, um wie viel die eine Helligkeit größer ist als die andere, so daß man nicht imstande ist, „bezüglich der Helligkeitsgrade ein anderes Verhältnis zu entscheiden, als eben das Verhältnis der Gleichheit". Hierbei läBt sich indessen keine vollkommene Gleichheit erreichen, da es minimale Differenzen der objektiven Helligkeit gibt, die sich der Wahrnehmung entziehen.

Darum ist eine experimentelle Prüfung der Helligkeitsunterscheidung vorzunehmen. $\mathrm{Zu}$ diesem $\mathrm{Zwecke}$ beleuchtet Lambert eine weiße ebene Fläche durch eine Kerze, so daß von einem mittleren Punkte aus die objektive Lichtstärke in mathematisch bestimmbarer Weise abnimmt, um nun die Grenze festzustellen, innerhalb welcher die objektiv vorhandene Differenz der Lichtstärke nicht wahrnehmbar ist. Er weist auf diese Weise nach, daß diese Differenz tatsächlich klein ist; er findet aber - wohl infolge der ungeeigneten Einrichtung der Versuche - nicht ein konstantes, sondern ein bei zunehmender Distanz der Kerze von 0,04 bis 0,07 anwachsendes Verhältnis der eben merklichen zur vorhandenen Lichtintensität.

In gleicher Weise, wie Lambert, sieht sich sein Zeitgenosse Bouguer zu einer Prüfung der Unterscheidbarkeit gegebener Lichtstärken veranlaßt. In seinem nachgelassenen, 1760 veröfentlichten photometrischen Werke stellt er an die Spitze aller Beobachtungen diejenigen über den Grad der Stärke, den ein Licht haben muß, um die Wirkung eines anderen Lichtes unmerklich zu machen. Denn - so sagt Bouguer ${ }^{2}$ ) - „ebenso wie ein starkes Geräusch uns hindert, ein anderes schwächeres zu hören, so sehen wir auch in Gegenwart eines hellen Lichtes ein anderes von viel geringerer Intensität nicht, wenn beide die nämliche Stelle der Netzhaut treffen". In der Tat findet er, daß eine vorhandene Lichtstärke um den 64 . Teil vermehrt werden muß, wenn der Zuwachs merklich werden soll.

1) Traité d'optique sur la gradation de la lumière, p. 51 (1760). 
Bouguer und Lambert untersuchten somit die Fähigkeit des Auges zur Unterscheidung von Helligkeiten, indem sie die Grenzen ermittelten, innerhalb welcher. die Verstärkung oder Schwächung einer vorhandenen Lichtstärke nicht wahrnehmbar ist.

Auf einem anderen Wege suchte Steinheil in der Abhandlung „Elemente der Helligkeitsmessungen am Sternhimmel (1837) ein Maß für die Schärfe der Helligkeitsunterscheidung zu gewinnen. Er hatte ein Prismenphotometer konstruiert, das die Herstellung gleicher, im Gesichtsfelde benachbarter Helligkeiten verlangte. Um nun den Grad der Sicherheit kennen zu lernen, der bei der Herstellung gleicher Helligkeiten erreichbar ist, führte er mit seinem Instrumente für vier verschiedene Helligkeiten Messungen aus und berechnete für jede Beobachtungsreihe den wahrscheinlichen Fehler. Diese wahrscheinlichen Fehler betrachtete er als Maß für die Unterscheidbarkeit der Lichtstärken. Er fand so, daß man den Helligkeitsunterschied zweier Lichtstärken noch wahrnehmen könne, wenn die eine um den 38. Teil von der anderen verschieden sei, wonach er zwar nicht zu demselben Zahlenwerte, aber'doch zu der nämlichen Gesetzmäßigkeit wie Bou guer gelangte.

Diese durch die Bedürfnisse der Photometrie hinsichtlich der Helligkeitsempfindungen geforderten Untersuchungen auf die anderen Sinnesgebiete auszudehnen, mußte als eine Aufgabe der Physiologie erkannt werden, sobald die Vorbedingungen für sinnesphysiologische Untersuchungen erfüllt waren. Dies war zur Zeit Lamberts und Bouguers, als Haller in den Jahren 1757 bis 1766 die "Elementa Physiologiae corporis humani“ schrieb, noch nicht der Fall. Damals war die Physiologie von der Auffassungsweise Descartes' beherrscht, nach welcher mechanische Bewegungsvorgänge von der Außenwelt sich bis ins Gebirn fortpflanzen, während die Seele auf Grund ihrer besonderen Beschaffenheit und ihres Urteilsvermögens aus diesen Einwirkungen etwas ganz Neues hervorbringt und zu deutlicher Klarheit erhebt. Dabei konnte es allerdings den Sinnen gelingen, die Seele $z u$ täuschen und zu irrtümlichen Vorstellungen zu veranlassen; an einen gesetzmäßigen, experimentell nachweisbaren Zusammenhang zwischen den Einwirkungen auf die Seele und den Gegenwirkungen derselben dachte man aber nicht. 
Eine physiologische Auffassungsweise zu entwickeln und zur Geltung zu bringen, war Johannes Müller (1801 bis 1858) vorbehalten, der zur Erkenntnis gelangte ${ }^{1}$ ), „daß Licht, Farbe, 'Ton, Wärme, Kälte und die verschiedenen Gerüche und Geschmäcke, mit einem Worte, was alles uns die fünf Sinne an allgemeinen Eindrücken bieten, nicht die Wahrheiten der äußeren Dinge, sondern die reellen Qualitäten unserer Sinne sind". Er überwand so die "physikalische" Periode der Physiologie, „in welcher die eigentlich physiologische Frage nicht weiter gehọrt wurde, die Physiologen das physikalische Resultat, als wäre damit genug getan, ohne weiteres willig auch als physiologisch aufnahmen".

Nachdem so die Besonderheit und Selbständigkeit der Nervenerregung zur Anerkennung gekommen war, konnte auch die Untersuchung der Abhängigkeit zwischen Sinnesreiz und Sinnesempfindung als eine physiologische Aufgabe erkannt werden.

E. H. Weber nahm in seinen Untersuchungen über den Tastsinn (1830 bis 1834) diese Aufgabe in Angriff. Er machte Versuche über die richtige Auffassung der Distanz zweier Gegenstände, welche das Tastorgan gleichzeitig berühren, und über die Feinheit des Tastsinnes bei der Wabrnehmung von Gewichten und bei der Empfindung von Wärme. Die Distanzauffassung prüfte er mittels eines Zirkels, dessen Spitzen so weit voneinander entfernt oder einander genähert wurden, daß sie eben unterscheidbar waren oder wenigstens die Richtung, nach der sie auseinander gingen, noch wahrgenommen werden konnte. Die bierbei sich ergebende Entfernung der Zirkelspitzen diente als $\mathrm{Maß}$ für die Feinheit der räumlichen Wahrnehmung an den verschiedenen Stellen der Leibesfläche. - Auch für die Wabrnehmung von Gewichten stellt er den eben merklichen Unterschied fest, indem er sowohl von zwei nacheinander auf eine und dieselbe Stelle der Haut aufgelegten Gewichten als auch von zwei nacheinander gehobenen Gewichten das eine oder das andere vermehrte oder verminderte, bis die Grenze, bei der die beiden Gewichte eben noch unterscheidbar waren, erreicht war. Außerdem belastete er zwei verschiedene Stellen der Leibesfläche, z. B. Lippen und Stirn oder Lippen und Finger, gleichzeitig mit Gewichten, von

1) Zur vergleichenden Physiologie des Gesichtssinnes des Menschen und der Tiere, S. 13, 18, 50, 65 (1826). 
welchen er das eine oder das andere stufenweise veränderte, bis beide gleich schwer zu sein schienen. - Die Unterscheidbarkeit von Wärmeempfindungen ferner untersuchte er, indem er die Hand abweclıselnd in zwei mit Wasser gefüllte Gefäße eintauchte und die Temperatur der Wassermengen durch Erhöhen oder Erniedrigen auf einen eben merkbaren Unterschied brachte.

Weber stellte demgemäß durch Abstufen objektiver Reize den eben merklichen Reizunterschied fest und nahm ihn als Maß für die Feinheit des Tastsinnes bei der Auffassung von Distanzen, sowie bei der Unterscheidung von Gewichten und von Wärmeempfindungen in Anspruch. Durch diese Versuche kam er zu der Erkenntnis, daß man bei der Beobachtung von Unterschieden nicht den Unterschied unmittelbar, sondern das Verhältnis des Unterschiedes zur Größe der miteinander verglichenen Gegenstände wahrnimmt. Er entdeckte so das Gesetz, das Fechner als die Hauptstütze seines psychophysischen Grundgesetzes (S. 48 und 49) ansah und nach seinem Entdecker das "Webersche Gesetz" (S. 50) genannt hat.

Außerdem bestimmte Weber für die Wahrnehmung von Gewichten die Reizgrößen, die bei gleichzeitiger Entwickelung an verschiedenen Körperteilen gleich orschienen und benutzte sie als vergleichendes Maß für die Empfindlichkeit der betreffenden Körperteile.

Durch seine sinnesphysiologischen Untersuchungen war Weber auch auf sonstige, die Feststellung objektiver Zustände und Vorgänge bedingende subjektive Faktoren aufmerksam geworden. Er erkannte den Einfluß der Ermüdung, der sich darin zeigt, daß Verschiedenheiten, die im ausgeruhten Zustande deutlich wahrgenommen werden, bei ermüdeten Sinnen unbeachtet bleiben. Er bemerkte auch, daß nacheinander einwirkende Reize schärfer unterschieden werden als gleichzeitig dargebotene. Den hierin zutage tretenden Einfluß der zeitlichen Ordnung auf die Unterscheidbarkeit der Empfindungen stellte er durch besondere Versuche fest, indem er gleichzeitige und unmittelbar aufeinanderfolgende, sowie durch verschieden lange Zeitintervalle getrennte Empfindungen miteinander verglich. Er machte so die ersten Gedächtnisversuche, die er der Beachtung der Psychologen empfahl, „da man so selten Gelegenheit hat, über solche geistigen Vorgänge Messungen zu machen". 
Während diese subjektiven Faktoren von dem ausgeruhten oder ermüdeten Zustande der Sinnesorgane und der zeitlichen Folge der Empfindungen abhängen und im allgemeinen bei jedem Beobachter in wechselndem Grade sich geltend machen können, war es eine der Persönlichkeit als solcher anhaftende Besonderheit, die bei astronomischen Beobachtungen von Bessel festgestellt und untersucht wurde.

Unerklärliche Abweichungen zwischen den von verschiedenen Beobachtern der Greenwicher Sternwarte festgestellten Durchgangszeiten der Sterne durch den Meridian veranlaßten Bessel, im Winter 1820/21 abwechselnd mit Walbeck am Meridiankreise der Königsberger Sternwerte den Durchgang einer Gruppe von zehn Sternen nach der damals üblichen, von Bradley eingeführten Auge- und Ohrmethode zu beobachten. Es galt hierbei, die Schläge des Sekundenpendels zu zählen und gleichzeitig den im Gesichtsfelde des Fernrohrs sich bewegenden Stern im Auge zu behalten, um die Stellung desselben bei zwei aufeinanderfolgenden Pendelschlägen unmittelbar vor und nach dem Durchgange durch den Mittelfaden zu erfassen und hiernach die Durchgangszeit selbst bis auf Zehntel Sekunden abzuschätzen. Bezeichnet man die von Walbeck und Bessel gefundene Durchgangszeit durch $W^{\prime}$ und $B$, so wird das Mittel aus vier Versuchstagen mit je acht bis zehn Beobachtungen durch die sogenannte „persönliche Gleichung":

$$
W-B=1,041 \text { Sekunden, }
$$

angegeben. "Der große Unterschied war" - wie Bessel ") bemerkt - „schon am zweiten Tage auffallend und veranlaßte eine Vermehrung der Aufmerksamkeit auf die wahren Momente des Durchganges; allein diese änderte ihn nicht, und wir schlossen die Beobachtungen mit der Überzeugung, daß es beiden unmöglich sei, auch nur ein einziges Zehntel einer Sekunde anders zu beobachten." Bessel stellte ferner die persönliche Gleichung zwischen sich selbst und noch anderen Astronomen (Argelander und Struve) fest. Er beobachtete auch an Stelle des stetig sich bewegenden Sternes das plötzliche Erscheinen oder Verschwinden eines Gegenstandes, wobei sich geringere Differenzen zwischen den Beobachtern ergaben. Hieraus folgerte er, „daß

') Astronomische Beobachtungen, 8. Abteilung, 1822. 
kein Beobachter, selbst wenn er Bradleys Beobachtungsmethode auf das strengste zu befolgen glaubt, sicher sein kann, absolute Zeitmomente sicher anzugeben". Er sieht darin eine rätselhafte Erscheinung, deren erschöpfende Untersuchung zwar wünschenswert, aber kaum möglich sei, ninden die Operationen, von welchen der Unterschied herrührt, ohne unser Bewußtsein vor sich gehen".

Demnach gelangt Bessel zu der Erkenntnis, daß Vorstellungen für die subjektive Auffassung sich gleichzeitig oder in unmittelbarer Aufeinanderfolge darbieten können, während die den Vorstellungen zugrunde liegenden objektiven Vorgänge durch meßbare Zeiten getrennt sind.

Es kommt aber für Bessel ebenso wie für E. H. Weber zunächst nur darauf an, das Vorhandensein der in Frage kommenden subjektiven Besonderheiten nachzuweisen. Darum besteht noch gar kein Bedürfnis, besondere Methoden für die Untersuchung dieser auf der Persönlichkeit des Beobachters beruhenden subjektiven Faktoren zu entwickeln.

Vierter Abschnitt.

\section{Die psychophysischen Maßmethoden.}

\section{Der naturphilosophische Standpunkt Fechners und das psychophysische Grundgesetz.}

Die Berücksichtigung subjektiver Faktoren im Bereiche der naturwissenschaftlichen Forschung hätte ohne weiteres eine vollständige und systematische Untersuchung des subjektiven Erfassens und Unterscheidens objektiver Zustände und Vorgänge veranlassen können. Dann wäre das Experiment, das bis dahin ausschließlich naturwissenschaftlichen Interessen gedient hatte, in den Dienst der Psychologie getreten und zum psychologischen Experiment geworden. Zugleich hätte sich das Bedürfnis geltend 
gemacht, an Stelle der primitiven Untersuchungsweise, die zur Feststellung der Mängel in der Sinneswahrnehmung und sonstiger, die Beobachtung des objektiven Tatbestandes störender Faktoren zunächst genügte, Methoden zu entwickeln, die für alle Anforderungen der experimentellen Psychologie ausreichend gewesen wären.

Dies war indessen nicht der Fall. Es wurde vielmehr vorerst nur ein Ausschnitt aus dem Gesamtgebiete der experimentellen Psychologie in den von naturphilosophischen Ideen getragenen "Elementen der Psychophysik" (1860) durch G. Th. Fechner (1801 bis 1887) der Forschung erschlossen. Hierbei waren die auf naturwissenschaftlicher Seite bereits vorliegenden Anfänge zunächst unbeachtet geblieben. Sie wurden darum durch Fechner nachträglich nur insoweit herangezogen, als sie auf jenes Teilgebiet Bezug hatten und den Interessen der Psychophysik dienstbar gemacht werden konnten. Desgleichen wurden die zur Ermittelung der subjektiven Faktoren benutzten Untersuchungsweisen ausschließlich in den Dienst der Psychophysik gestellt und zu psychophysischen Maßmethoden ausgebildet.

Fechner war nämlich durch die aus begrifflichen Konstruktionen bestehenden, von quantitativen naturgesetzlichen Bestimmungen sich fernhaltenden Bearbeitungen der Naturphilosophie, die Schelling, Hegel und ihre Nachfolger gegeben hatten, unbefriedigt geblieben. Er wollte im Gegensatz hierzu vom Gebiete der Erfahrungswissenschaft aus durch Induktion und Analogieschlüsse zu höchsten und letzten Gesichtspunkten gelangen. Insbesondere war es das Gebiet der Beziehungen zwischen Körper und Geist, das seine Interessen gefangen nahm, und von dessen erfolgreicher Erforschung er die Lösung aller Rätsel des Menschenlebens und des Weltgeschehens erhoffte. So wurde für ihn die Frage nach dem Zusammenhange von Leib und Seele zur Grundfrage der Philosophie. Sie fand ibre Beantwortung durch die Erkenntnis, daß Leib und Seele wechselweise sich bedingen, und $\mathrm{da} B$ die Seele die Einheit gegenüber der leiblichen Mannigfaltigkeit darstelle. Darum betrachtete Fechner die Seele als die einheitliche Selbsterscheinung dessen, was äußerlich als die Mannigfaltigkeit des Leibes erscheine; und er sah sich so dazu geführt, in analoger Weise ein allgemeines Weltbewußtsein anzunehmen, das alle Erscheinungen, die es gibt, in sich verknüpft und sich 
in untergeordnete Bewußtseinseinheiten, zu denen auch die Seele des Menschen gehört, entfaltet.

Dieses Weltbild erhält demnach durch die tatsächlich vorhandene Beziehung zwischen dem Leibe und der Seele des Menschen eine empirische, der Untersuchung zugängliche Unterlage. Darum tritt für Fechner die Psychophysik als exakte Lehre von der Abhängigkeit zwischen Leib und Seele in den Mittelpunkt der wissenschaftlichen Bestrebungen. Es muß ja die auf Erfahrung gegründete, durch $\mathrm{Maß}$ und Zahl gestützte Erforschung jener Abhängigkeit zugleich das allgemein gültige Gesetz des Zusammenhanges zwischen materiellem und geistigem Prinzip überhaupt erkennen lassen - in ähnlicher Weise, wie für Newton die durch Rechnung konstatierte Gravitation zwischen Erde und Mond die Aufstellung des alle Himmelskörper umspannenden Gravitationsgesetzes möglich gemacht hat.

$\mathrm{Daß}$ die Abhängigkeit zwischen Seele und Leib durch eine mathematische Funktion darstellbar sei, hielt Fechner für selbstverständlich. Nur die Form der Funktion stand in Frage. Zuerst dachte er an eine Proportionalität zwischen der Intensität der geistigen Tätigkeit einerseits und der lebendigen Kraft der zugrunde liegenden körperlichen Tätigkeit andererseits. Nachdem er aber sich gewöhnt hatte, die Beziehung zwischen Leib und Seele und zwischen niederem und höherem Geistigen durch die Gegenüberstellung von arithmetischen Reihen niederer und höherer Ordnung oder von arithmetischen und geometrischen Reihen zu erläutern, sab er sich durch das Schema der geometrischen Reihe dazu geführt, den verhältnismäßigen $\mathrm{Zuwachs}$ der körperlichen lebendigen Kraft dem direkten Zuwachs der geistigen Tätigkeit proportional zu setzen, so daß

$$
d y=c \frac{d x}{x} \text {. }
$$

wenn $y$ die geistige Tätigkeit, $x$ die zugehörige körperliche Tätigkeit und $c$ eine Konstante bezeichnet. Hieraus ergab sich durch Integration das psychophysische Grundgesetz:

$$
y=c . \lg x \text {. . . . . }
$$

wonach die geistige Intensität dem Logarithmus der zugehörigen körperlichen lebendigen Kraft proportional ist. 
Trifft dies zu, so muß sich durch Beobachtung feststellen lassen, daß beliebige Unterschiede der geistigen Intensität den Logarithmen der Verhältnisse aus der zugehörigen körperlichen lebendigen Kraft, und hinreichend kleine Unterschiede der geistigen Intensität den relativen Unterschieden der zugehörigen körperlichen lebendigen Kraft proportional sind.

Ist nämlich

so ist

$$
y_{1}=c . \lg x_{1} ; \quad y_{2}=c . \lg x_{2}
$$

$$
y_{2}-y_{1}=c . \lg \frac{x_{2}}{x_{1}} \cdot \text {. . . . . }
$$

Ist überdies die Differenz $y_{2}-y_{1}$ hinreichend klein, so ist auch $x_{2}: x_{1}$ nahezu gleich 1 . Setzt man nun

$$
\begin{gathered}
\frac{x_{2}}{x_{1}}=1+\delta, \\
\lg (1+\delta)=\delta-1 / 2 \delta^{2}+1 / 3 \delta^{3}-1 / 4 \delta^{4}+\cdots,
\end{gathered}
$$

so kann man für hinreichend kleine Werte $\delta$ die zweiten und höheren Potenzen vernachlässigen, so daß $l g(1+\delta)=\delta$ und

$$
y_{2}-y_{1}=c . \delta
$$

oder, da $\delta=\left(x_{2}-x_{1}\right): x_{1}$,

$$
y_{2}-y_{1}=c \frac{x_{2}-x_{1}}{x_{1}}
$$

\section{Das Maß der Empfindlichkeit.}

Zur Prüfung dieser Gesetzmäßigkeiten konnte die Untersuchung zwischen Reiz und Empfindung dienen, sofern gleiche Intensitäten und gleiche Intensitätsunterschiede der Empfindungen feststellbar sind. Die Fähigkeit, Reize oder Abänderungen von Reizen in bestimmter Stärke wahrzunehmen, wird aber als Empfindlichkeit, und zwar, sofern absolute Reize in Betracht kommen, als absolute Empfindlichkeit, sofern es sich um Reizunterschiede handelt, als Unterschiedsempfindlichkeit bezeichnet.

Es galt demnach die Empfindlichkeit in ihren beiden Formen als absolute Empfindlichkeit und als Unterschiedsempfindlichkeit messend zu verfolgen, um die Abhängig- 
keit zwischen Empfindungsintensität und Reizstärke und hierdurch zwischen geistiger Intensität und körperlicher lebendiger Kraft zu bestimmen. Dabei hat man die Empfindlichkeit dem reziproken Werte des in bestimmter Stärke empfundenen Reizes oder Reizunterschiedes proportional zu setzen, da man die Empfindlichkeit um so größer zu nennen pflegt, je kleiner der in bestimmtem Maße empfundene Reiz oder Reizunterschied ist.

Es wird indessen ein und derselbe Reiz oder eine und dieselbe Reizabänderung unter sonst gleichen Umständen von verschiedenen Individuen oder von demselben Individuum zu verschiedenen Zeiten verschieden stark empfunden. Hierdurch wird aber die Gewinnung brauchbarer Maßwerte der Empfindlichkeit nicht unmöglich gemacht, sondern nur die Verpflichtung auferlegt, besondere Methoden zur Messung der Empfindlichkeit aufzustellen und zu befolgen.

Es sind dies die psychophysischen Maßmethoden. Sio sollen die Bestimmung des psychophysischen Grundgesetzes ermöglichen. Dabei dienen sie aber, eben weil sie die Schwankungen, in der Empfindlichkeit und somit die auf der Ungenauigkeit der Sinneswahrnehmung beruhenden subjektiven Faktoren berücksichtigen, zugleich den Interessen der experimentellen Psychologie. Und wenn auch die Absicht nicht unmittelbar auf das Studium, sondern vielmehr auf die Elimination dieser subjektiven Faktoren gerichtet ist, so findet trotzdem - dank der großen Sorgfalt, mit der Fechner bei seinen Untersuchungen verfährt - der die Sinneswahrnehmung betreffende Teil der experimentellen Psychologie eine ausgiebige Pflege. Zugleich gewinnt so die Psychophysik einen Anschluß an die auf naturwissenschaftlichem Gebiete bereits vorhandenen Bestrebungen, die Genauigkeit der Sinneswahrnehmung festzustellen, wobei namentlich die Untersuchungsergebnisse von E. H. We ber und insbesondere das von Weber gefundene und nach ihm benannte, die Grundformel (20) bestätigende Gesetz in den Vordergrund treten.

Für die vorzugsweise zur Ausführung gelangende Bestimmung der Unterschiedsempfindlichkeit wurden von Fechner drei Methoden unterschieden: die Methode der eben merklichen Unterschiede, der mittleren Fehler und der richtigen und falschen Fälle. Bei der Methode der eben merk- 
lichen Unterschiede werden eben merklich erscheinende Reizunterschiede als gleichempfundene Unterschiede angesehen, so daß die Unterschiedsempfindlichkeit dem reziproken Werte des eben merklichen Reizunterschiedes proportional zu setzen ist. Die Methode der mittleren Fehler ferner mißt die Unterschiedsempfindlichkeit durch den reziproken Wert des durchschnittlichen Fehlbetrages, der bei wiederholten Versuchen, zwei Reizgrößen nach der Empfindung einander gleich zu machen, entsteht. Nach der Methode der richtigen und falschen Fälle schließlich werden zwei wenig voneinander verschiedene Reizgrößen in oftmaliger Wiederholung dem Urteil dargeboten. Man wird alsdann den Reizunterschied bald richtig auffassen, bald über die Richtung des Unterschiedes sich täuschen, so daß man in einer großen Anzahl von Versuchen ein bestimmtes Verhältnis der richtigen und der falschen Fälle zur Gesamtzahl der Fälle erhält. Nun ist die Empfindlichkeit offenbar um so geringer, jo größer der Reizunterschied sein muß, um gleich oft in einer bestimmten Anzahl von Fällen richtig beurteilt zu werden. Darum ist nach Wahl eines bestimmten Verhältnisses der richtigen Fälle zur Gesamtzahl aller Fälle die Unterschiedsempfindlichkeit dem reziproken Werteder Reizdifferenz, bei der jenes Verhältnis erreicht wird, proportional.

Diesen Methoden stellt Fechner drei analoge Methoden zur Feststellung der absoluten Empfindlichkeit zur Seite: die Bestimmung des eben merklichen Reizes, wobei die Empfindlichkeit durch den reziproken Wert des eben merklichen Reizes gemessen wird; sodann die als Methode der Äquivalente bezeichnete Herstellung von Reizen, die einem anderen, unter vergleichbaren Umständen (etwa an einer anderen Stelle der Hautfläche) wirkenden Reize gleich stark zu sein scheinen; schließlich die Ermittelung der Anzabl richtiger und falscher Fälle bei der Beurteilung kleiner, an der Grenze der Merklichkeit liegender Reizgrößen.

\section{Die Methode der eben merklichen Unterschiede.}

Von den drei Maßmethoden der Unterschiedsempfindlichkeit ist die an erster Stelle genannte Methode der eben merklichen 
Unterschiede nach Fechners Auffassung die einfachste und direkteste, da sie wenig Rechnung nötig macht und schon bei einer geringen Anzahl von Versuchen zu einem Ergebnis führt. Darum ist sie auch bei den Bestimmungen der Sinnesschärfe in Gebiete der Photometrie und Physiologie vorzugsweise zur Anwendung gekommen und namentlich von E. H. Weber benutzt worden. Sie leidet indessen, wie $\mathrm{Fechn} \theta \mathrm{r}$ hervorhebt, an der Unbestimmtheit, die der Feststellung des eben Merklichen anhaftet, da nicht eine scharfe Grenze, sondern ein Intervall des Zweifels die unmerklichen von den merklichen Reizunterschieden trennt. Darum müsse man einen zwar kleinen, aber noch sicher auffaßbaren Empfindungsunterschied festzuhalten und bei den wiederholten Versuchen zu reproduzieren versuchen, um aus dem Mittel der so sich ergebenden Reizunterschiede den gesuchten eben merklichen Reizunterschied zu erhalten.

Das Festhalten eines zwar kleinen, aber bestimmt auffaßbaren Empfindungsunterschiedes läßt sich indessen nicht mit Sicherheit ausführen. Darum wurde das von Fechner angegebene Verfahren durch G. E. Müller ${ }^{1}$ ) und Wundt ${ }^{2}$ ) umgestaltet.

Nach G. E. Müller ist ein deutlich übermerklicher Reizunterschied allmählich zu schwächen, bis er nicht mehr merkbar ist, und ein untermerklicher Reizunterschied allmählich zu verstärken, bis er eben merkbar wird. Das arithmetische Mittel aus gleich vielen, auf diese Weise erhaltenen Bestimmungen des eben merklichen und des eben unmerklichen Reizunterschiedes bietet alsdann den gesuchten Wert dar. - Wundt hingegen benutzt an Stelle der allmählichen Verstärkung und Schwächung minimale, aber diskrete Änderungen des Reizunterschiedes, so da ß eine vorbestimmte Stufenfolge der Reizwerte durchlaufen und was von besonderer Bedeutung ist - an Stelle der regelmäßigen, durch alle $Z$ wischenstufen nacheinander führenden Variation eine unregelmäßige, durch sprungweises Vorwärts - und Rückwärtsgehen die Stufenfolge ausfüllende Variation der Reizwerte treten kann. Dieses Verfahren wird mit Rücksicht auf die Verwendung $1,558$.

1) Zur Grundlegung der Psychophysik 1879, S. 63.

2) Über die Methode der Minimaländerungen, Philos. Studien 
minimaler Änderungen der Reizwerte auch die Methode der Minimaländerungen genannt.

In dem Mittelwerte, den man aus den auf diese Weise beobachteten eben merklichen und eben unmerklichen Reizunterschieden erhält, hofft man den Grenzwert zu treffen, der das Gebiet der merklichen Unterschiede von dem Gebiete der unmerklichen Unterschiede trennt und demgemäß als Unterschiedsschwelle bezeichnet wird. $\mathrm{Zu}$ jedem Reizwerte $r$ findet man so einen, das Gebiet der merklich stärkeren Reize abgrenzenden Wert $r_{o}$ und einen, zu den merklich schwächeren Reizen führenden Wert $r_{u}$, wonach $\Delta r_{0}=r_{0}-r$ die obere und $\Delta r_{u}=r-r_{u}$ die untere Unterschiedsschwelle angibt. Den Wert $\Delta r$ $=\left(\Delta r_{o}+\Delta r_{u}\right): 2$ nennt Wundt die mittlere Unterschiedsschwelle. Er bezeichnet ferner den Wert $R=r_{o}-\Delta r$ $=r_{u}+\Delta r$ als Schätzungswert des Reizes $r$ und nennt die Differenz $\Delta=R-r=\left(\Delta r_{o}-\Delta r_{u}\right): 2$ die Schätzungsdifferenz, die durch einen positiven Wert ein Überschätzen und durch einen negativen Wert ein Unterschätzen des Reizes andeutet.

Indem man aus den beobachteten Werten das arithmetische Mittel berechnet, um die Unterschiedsschwelle zu bestimmen, gelangt der bei astronomischen und physikalischen Messungen festgehaltene Grundsatz zur Anerkennung, daß im arithmetischen Mittel der wahre Wert einer beobachteten Größe mit größter Wahrscheinlichkeit sich darbiete. Es wird demnach vorausgesetzt, daß ein wahrer Unterschiedsschwellenwert vorhanden sei, und daß dieser Wert wie eine objektiv existierende Größe gemessen werden könne. Es wird aber nicht bedacht, da $b$ diese Voraussetzung erst geprüftwerden muß.

$\mathrm{Zu}$ diesem Zwecke ist die ursprüngliche Methode Fechners und ihre durch G. E. M üller angeregte Modifikation von der durch Wundt eingeführten Umgestaltung wohl zu unterscheiden.

Die Unterschiedsschwelle trennt die nicht bemerkbaren Unterschiede von den bemerkbaren. Sie wird somit durch denjenigen Reizwert bezeichnet, der im Vergleich mit dem gegebenen Reizwerte weder als merkbar gleich noch als merkbar verschieden erscheint, sondern den Zustand des Zweifels, ob Gleichheit oder Verschiedenheit vorhanden sei, erweckt. Man kann daher offenbar von einem direkten Messen der Unterschiedsschwelle reden, wenn man einen beliebig und willkürlich veränderlichen Ver- 
gleichsreiz während seiner Einwirkung auf das Sinnesorgan bei gleichzeitiger Einwirkung des Hauptreizes so verändert, daß jener Zustand des Zweifels eintritt, und alsdann den gefundenen Reizwert mißt. Es kann ferner gleichfalls als ein Messen der Unterschiedsschwelle gelten, wenn man mit Fechner einen zwar kleinen, aber noch sicher auffaßbaren Empfindungsunterschied zu gewinnen sucht, oder wenn man mit G. E. Müller, um die bei diesem Verfahren unvermeidliche Vergrößerung des Unterschiedsschwellenwertes zu vermeiden, sowohl die eben merklichen als auch die eben unmerklichen Reizunterschiede bestimmt. In jedem Falle wird man geneigt sein, nach wiederholter Ausführung der Messung das arithmetische Mittel der Maßwerte für den wahren Wert $\mathrm{zu}$ halten und die mittlere Abweichung von dem arithmetischen Mittel als ein Maß für die erreichte Genauigkeit zu betrachten. Dies ist aber nur dann gestattet, wenn die Beobachtungen von sogenannten konstanten Fehlern frei sind. $\mathrm{Da}$ jedoch in der Regel konstante Fehler in beträchtlicher Größe auftreten, so ist das von Fechner angegebene Verfahren sowohl in der ursprünglichen wie auch in der durch G. E. Müller verbesserten Form zu einer zuverlässigen Bestimmung der Unterschiedsschwelle nicht geeignet.

Um dies an einem Beispiele klar zu legen, habe ich mit rotierenden, aus weißen und schwarzen Sektoren bestehenden Scheiben Helligkeitsvergleiche angestellt. Eine Scheibe, deren Sektoren während der Rotation verstellt werden konnten, wurde so eingestellt, daß sie eben heller oder eben dunkler als eine daneben rotierende Scheibe mit fest bestimmten unveränderlichen Sektoren erschien. Für das Grau einer aus $180^{\circ}$ Weiß und $180^{\circ}$ Schwarz bestehenden Normalscheibe ergab auf diese Weise die verstellbare Vergleichsscheibe ein merklich helleres Grau, wenn sie im Mittel von 100 Versuchen auf 202,5 Weiß und $157,5^{\circ}$ Schwarz eingestellt wurde, wobei die mittlere quadratische Abweichung gleich $\pm 0,9^{\circ}$ war. Die Vergleichsscheibe war dagegen merklich dunkler, wenn ihre Sektoren in 100 Versuchen durchschnittlich aus $154,5^{\circ}$ Weiß und $205,5^{\circ}$ Schwarz mit der mittleren quadratischen Abweichung $\pm 0,7^{\circ}$ bestanden. Die obere Unterschiedsschwelle wäre demnach gleich $22,5^{\circ}$, die untere gleich $25,5^{0} \mathrm{zu}$ setzen, wenn man davon absehen wollte, daß diese Be- 
stimmungsweise notwendig einen zu großen Wert ergibt. Daß nun die Verschiedenheit der beiderseitigen Unterschiedsschwellenwerte durch einen konstanten Fehler veranlaßt wird, tritt deutlich zutage, wenn man die Versuche, durch welche die Vergleichsscheibe auf die nämliche Helligkeit wie die Normalscheibe gebracht wurde, berücksichtigt. Aus 200 Versuchen ergab sich nämlich, daß die Vergleichsscheibe der Normalscheibe gleich hell erschien, wenn sie durchschnittlich aus $178,5^{\circ} \mathrm{Wei}$ und $181,5^{\circ}$ Schwarz bestand. Legt man diesen Wert zugrunde, so findet man beiderseits $24^{\circ}$ als Unterschiedsschwellenwert. Auf diese Weise wird aber nur derjenige Teil des konstanten Fehlers eliminiert, der die Differenz des oberen und unteren Unterschiedsschwellenwertes bedingt. Man hat hingegen kein Mittel, um der beiderseitigen Vergrößerung dieses Wertes zu begegnen. Und auch dann, wenn man die Mittelwerte für die verschwindenden Helligkeitsunterschiede hinzunimmt, wodurch sich der gefundene Wert auf höchstens $12^{\circ}$ reduziert, ist keine Gewähr vorhanden, daß nicht doch noch eine Vergrößerung oder Verkleinerung des wahren Wertes vorliegt. In der Tat ist hier eine Vergrößerung anzunehmen, da eine auf ganz anderen Grundsätzen beruhende einwandfreie Bestimmung der Unterschiedsschwelle (in $\S 20$ ) aus den Einstellungen auf gleiche Helligkeit $11^{\circ}$ als obere Grenze, die nicht erreicht werden kann, finden läßt.

Ein Messen der Unterschiedsschwelle findet hingegen nicht statt, wenn nach dem durch Wu ndt vorgeschlagenen, allgemein in Aufnahme gekommenen Verfahren eine vorbestimmte Skala von Reizwerten in regelmäßigem Auf - und Absteigen oder in unregelmäßigem Wechsel dargeboten wird. Denn nun kann der Beobachter nicht den Reizwert während der Einwirkung auf das Sinnesorgan verändern, bis der Zustand des Zweifels an Gleichheit oder Verschiedenheit eintritt, oder bis ein nicht vorhandener Unterschied merkbar oder ein vorhandener Unterschied unmerkbar wird. Er muß vielmehr bei jedem dargebotenen Vergleichsreize das Vorhandensein von Gleichheit oder Verschiedenheit, oder auch den Zweifel, ob Gleichheit oder Verschiedenheit bestehe, feststellen. Hierbei wird es violfach zutreffen, daß beim Durchlaufen der Skala der Vergleichsreize von den kleineren Werten aus an einer bestimmten Stelle das Urteil "kleiner" $(<)$ durch das Urteil "gleich" (二) und das letztere an einer höher 
gelegenen Stelle durch das Urteil "größer" $(>)$ abgelöst wird. Es kann aber auch nicht selten nach erfolgter Ablösung ein Rückschlag in die frühere Urteilsart erfolgen oder wenigstens ein Zweifel, ob die eine oder die andere Urteilsweise richtig sei, sich regen, so daß man sich mit den Urteilen "gleich" oder "größer" $(=>$ ) und "gleich" oder "kleiner" $(=<)$ zufrieden geben muß. Es ist ferner möglich, $\mathrm{da}$ ein direkter Übergang der Urteile "kleiner" zu den Urteilen "größer" ohne eine Vermittelung durch das Urteil "gleich" sich ergibt.

Beispielsweise gelangte ich durch Helligkeitsvergleichungen unter Zugrundelegen einer aus 11 Stufen bestehenden Skala von Vergleichsreizen zu den in Tabelle 2 angegebenen Urteilsreihen.

Tabelle 2.

\begin{tabular}{c||c|c|c|c|c|c|c|c}
\hline \hline & $u$ & $r$ & $u$ & $u$ & $r$ & $u$ & $r$ & $u$ \\
\hline \hline 1. & $<$ & $<$ & $<$ & $<$ & $<$ & $<$ & $<$ & $<$ \\
2. & $<$ & $<$ & $<$ & $<$ & $<$ & $<$ & $<$ & $=$ \\
3. & $<$ & $<$ & $<=$ & $<=$ & $<$ & $<$ & $<$ & $=$ \\
4. & $<$ & $<$ & $=$ & $=$ & $<$ & $<$ & $<=$ & $=$ \\
5. & $=$ & $=$ & $=$ & $=$ & $=$ & $<=$ & $=$ & $<$ \\
6. & $<=$ & $=$ & $=$ & $=$ & $=$ & $=>$ & $=$ & $>$ \\
7. & $=$ & $=$ & $=$ & $=$ & $=$ & $=$ & $=$ & $=$ \\
8. & $=$ & $>$ & $>$ & $>$ & $>$ & $=$ & $=$ & $>$ \\
9. & $>$ & $=$ & $=$ & $>$ & $>$ & $=$ & $=$ & $>$ \\
10. & $>$ & $>$ & $>$ & $>$ & $>$ & $>$ & $>$ & $>$ \\
11. & $>$ & $>$ & $>$ & $>$ & $>$ & $>$ & $>$ & $>$
\end{tabular}

Ein anderes Mal ergab sich bei einer Verdoppelung der Stufen der direkte Übergang der Urteile "kleiner" in die Urteile "größer" ohne dazwischentretende Urteile "gleich", so daß die Reihe

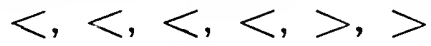

entstand. Ein über die Reihe gesetztes $r$ bedeutet, daß der Vergleichsreiz regelmäßig auf- und absteigend variiert wurde. Ein $u$ deutet die im allgemeinen den Vorzug verdienende unregelmäßige Variation des Vergleichsreizes an. Die eine oder die andere Art der Reizdarbietung erfolgte ohne Wissen des Beobachters. Als Hauptreiz diente das Grau einer rotierenden Scheibe, die aus $100^{\circ}$ Weiß und $260^{\circ}$ Schwarz bestand, während die $11 \mathrm{Ab}-$ 
stufungen des Vergleichsreizes der Reihe nach durch $86,5^{\circ}, 88^{\circ}$, $89,5^{\circ} \ldots 100^{\circ}, 101,5^{0}$ Weiß und $273,5^{\circ}, 272^{0}, 270,5^{\circ} \ldots 260^{\circ}$, $258,5^{\circ}$ Schwarz bestimmt wurden. Man ersieht hieraus, daß eine beträchtliche Überschätzung der Helligkeit der Vergleichsscheibe stattfand, die durch eine Kontrastwirkung bedingt war.

Der Anblick dieser Tabelle läßt ohne weiteres erkennen, daß unmittelbar aus den einzelnen Urteilsreihen keine einwandfreie Bestimmung der Unterschiedsschwelle gewonnen werden kann. Denn wenn auch bei einem regulären Verlaufe die Stellen, wo die Urteile "kleiner" in die Urteile "gleich" und die letzteren in die Urteile "größer" übergehen als untere und obere Unterschiedsschwellen in Anspruch genommen werden dürfen, so fehlt die Möglichkeit hierzu, sobald ein mehrfacher Übergang von der einen zur anderen Urteilsart wie in der 1., 2., 3., 6., 8. Reihe stattfindet. Die übliche Handhabung der Methode der Minimaländerungen beruht aber gerade auf der Benutzung der einzelnen Urteilsreihen. Sie ist darum zur Bestimmung der Unterschiedsschwellenwerte nicht geeignet. $\mathrm{Zu}$ einem in jedem Falle anwendbaren Verfahren gelangt man indessen durch folgende Überlegung.

Je mehr sich die Urteilsreihen in der Tabelle 2 häufen, um so deutlicher wird es, daß jeder Stufe die Urteile „kleiner", "gleich", "größer" mit einer bestimmten relativen Häufigkeit oder Wahrscheinlichkeit zukommen. Dies erhellt bereits aus der in Tabelle 3 zusammengestellten Anzahl der Urteile, die sich bei 19 maliger Darbietung jeder der 11 Helligkeitsstufen ergeben hat.

Tabelle 3 .

\begin{tabular}{r||r|r|r}
\hline \multicolumn{1}{c||}{$a$} & $n$ & $z^{\prime}$ & $p$ \\
\hline 1 & 19 & - & - \\
2 & 15 & 4 & - \\
3 & 11 & 8 & - \\
4 & 7 & 12 & - \\
5 & 4 & 13 & 2 \\
6 & 2 & 12 & 5 \\
7 & - & 16 & 3 \\
8 & - & 7 & 12 \\
9 & - & 7 & 12 \\
10 & - & 2 & 17 \\
11 & - & - & 19 \\
& & &
\end{tabular}


Es gehören hier zu jeder unter a verzeichneten Helligkeitsstufe $n$ Urteile "kleiner", $z^{\prime}$ Urteile "gleich" und $p$ Urteile "größer", so daß z. B. die 6. Stufe 2 mal dunkler, 12 mal ebenso hell und 5 mal heller wie das Grau der Hauptscheibe erschien.

Auf Grund dieser Häufigkeitswerte hat nun der einer bestimmten Stufe angehörende Vergleichsreiz $r^{\prime}$ für merklich größer oder merklich kleiner als der Hauptreiz $r$ zu gelten, wenn $r^{\prime}$ nicht nur unbedingt und in jedem Falle, sondern auch bloß in der Mehrzahl der Fälle größer oder kleiner als $r$ erscheint. Demgemäß bezeichnet die Differenz $\Delta r_{o}=r_{o}-r$ die obere Unterschiedsschwelle, wenn bei einem hinreichend oft wiederholten Vergleiche von $r_{o}$ mit $r$ sich ebenso oft das Urteil "größer" wie das Urteil "gleich" oder "kleiner" ergibt. Es gibt ferner die Differenz $\Delta r_{u}=r-r_{u}$ die untere Unterschiedsschwelle an, wenn das Urteil „kleiner" mit der gleichen Häufigkeit wie das Urteil "gleich" oder "größer" auftritt. Es ist folglich, wenn man 19 Urteilsreihen für genügend halten will, in der Tabelle 3 die obere Unterschiedsschwelle zwischen der 7. und 8. Stufe, die untere Unterschiedsschwelle zwischen der 3. und 4. Stufe zu suchen. Es bedarf aber kaum des besonderen Hinweises darauf, daß man die Versuche in starkem Maße häufen muß, bis die Stelle, wo die relative Häufigkeit der Urteile "größer" im oberen Gebiete und der Urteile "kleiner" im unteren Gebiete der Reizskala gleich $1: 2$ ist, mit hinreichender Deutlichkeit, die eine genaue, interpolatorische Bestimmung gestattet, hervortritt. Überdies müßte diese Bestimmung der Unterschiedsschwelle mehrfach wiederholt werden, damit ihre Sicherheit auf Grund der Abweichungen von dem gemeinsamen Mittelwerte beurteilt werden könnte. Denn sie repräsentiert, wie jede induktive Wahrscheinlichkeitsbestimmung, eine ganze Mannigfaltigkeit von Werten, die bei unbegrenzter Wiederholung der Beobachtungen hervortreten würde und durch die mittlere quadratische Abweichung der einzelnen Werte von ihrem arithmetischen Mittel angedeutet werden kann. Es ist auch das Auftreten konstanter Fehler in Rechnung zu ziehen, die durch die Häufung der Versuche keineswegs unmöglich gemacht werden.

Demnach führt die Methode der eben merklichen Unterschiede sowohl in der ursprünglichen wie auch in der umgestalteten Form zu keiner einwandfreien Be- 
stimmung der Unterschiedsschwelle, und somit auch zu keinem sicheren Maße der Unterschiedsempfindlichkeit.

\section{Die Methode der mittleren Fehler.}

Wenn der Reiz während seiner Einwirkung auf das Sinnesorgan durch den Beobachter in beliebiger und ungehinderter Weise verändert werden kann, so ist die Methode der mittleren Fehler anwendbar. Dies ist beispielsweise der Fall, wenn drei auf einem Maßstabe verschiebbare Fäden dem Auge dargeboten werden. Sucht man nun die Distanz des einen der beiden äußeren Fäden von dem mittleren Faden der unverändert bleibenden Distanz der beiden anderen Fäden gleich zu machen, so wird man hierbei im allgemeinen einen Fehler begehen. Die hergestellte Distanz, die Fehldistanz $F$, wird von der als Norm gegebenen Distanz, der Normaldistanz $N$, abweichen. Der Unterschied $F-N$ stellt den Fehler $\delta$ dar.

Hat man die Versuche in einer hinreichend großen Anzahl $m$ angestellt und die Fehldistanzen $F_{1}, F_{2}, F_{3} \ldots F_{m}$ mit den Fehlern $\delta_{1}, \delta_{2}, \delta_{3} \ldots \delta_{m}$ erhalten, so wird man erwarten, in dem arithmetischen Mittel der Fehldistanzen die zum Vergleich dienende Normaldistanz wieder zu finden. In dieser Erwartung sieht man sich indessen in der Regel getäuscht, indem das arithmetische Mittel um einen im allgemeinen nicht außer Acht zu lassenden Betrag $c$ von der Normaldistanz abweicht. Es würde somit erst dann, wenn die Fehldistanzen $F$ um den konstanten Betrag $c$ vermindert würden, das Mittel der verminderten $F$ mit $N$ übereinstimmen. Dann müßten auch die Fehler $\delta$ um den konstanten Betrag $c$ vermindert werden, wonach dieser Betrag als ein konstanter Bestandteil der Fehler $\delta$ und somit als ein konstanter Fehler aufzufassen ist.

Demgemäß kann der Fehler $\delta$ in den konstanten Fehler $c$ und einen variablen Bestandteil, den reinen variablen Fehler $\Delta$, zerlegt gedacht werden, so daß

$$
\delta_{1}=\Delta_{1}+c, \quad \delta_{2}=\Delta_{2}+c, \quad \delta_{3}=\Delta_{3}+c \ldots
$$

Der konstante Fehler $c$ ist wesentlich von den besonderen Bedingungen, unter denen die Versuche angestellt werden, abhängig. Man kann beispielsweise zuerst die Normaldistanz oder zuerst die Fehldistanz auffassen, oder auch die Richtung des 
Vergleichs beliebig wechseln. Hierdurch werden die Unterschiede der sogenannten Zeitlage bedingt. Man kann ferner entweder die nach der einen oder die nach der anderen Seite vom mittleren Faden aus sich erstreckende Distanz als Fehldistanz und die andere als Normaldistanz wählen, worauf die Unterschiede der sogenannten Raumlage beruhen. Außerdem hebt Fechner neben sonstigen unberechenbaren inneren Verhältnissen den „Einfluß der Herstellungsweise" der verglichenen Größen hervor, wobei er individuelle Eigentümlichkeiten im Auge hat, die den Grund dafür bilden, daß bei der Abänderung der Fehlgröße das richtige Maß überschritten oder nicht erreicht wird.

Diesen verschiedenartigen Einflüssen entsprechend setzt Fechner eine Zerlegbarkeit des konstanten Feblers voraus. Beispielsweise nimmt er an, daß die in den beiden Raumlagen sich ergebenden konstanten Fehler $c_{1}$ und $c_{2}$ aus einem von der Raumlage abhängenden und bei entgegengesetzter Lage sein Vorzeichen wechselnden Teile $\pm r$ und aus einem von der Raumlage unabhängigen Teile $u$ bestehen, und setzt demgemäß $c_{1}=r+u$, $c_{2}=-r+u$, woraus

$$
\left.\begin{array}{l}
r=1 / 2\left(c_{1}-c_{2}\right) \\
u=1 / 2\left(c_{1}-c_{2}\right)
\end{array}\right\} . \quad . \quad . \quad . \quad . \quad .
$$

sich ergibt. In entsprechender Weise sucht er den Einfluß der Zeitlage auf den konstanten Fehler zu bestimmen.

Die reinen Fehler $\Delta$ folgen, wie Fechner in Anlehnung an die von Gauss begründete Fehlertheorie annimmt, dem gewöhnlichen Fehlergesetze. Den aus den absoluten Beträgen $|\Delta|$ der reinen Fehler gebildeten Mittelwert

$$
\varepsilon=\frac{1}{m}\left\{\left|\Delta_{1}\right|+\left|\Delta_{2}\right|+\left|\Delta_{3}\right|+\cdots+\left|\Delta_{m}\right|\right\} .
$$

setzt er dem reziproken Werte der Unterschiedsempfindlichkeit proportional.

Daß hiermit kein unbedingt zuverlässiges Maß gewonnen wird, verkennt Fechner nicht. Er sagt in dem nachgelassenen Ianuskripte seiner Vorlesungen über die psychophysischen Maßmethoden ${ }^{1}$ ): "Bei aller Sorge, welche man treffen mag, die Versuchsumstände vergleichbar zu halten, ändern sich doch im

1) Im Fechner-Archiv d. Sächs. Ges. d. Wiss., Nr. 111, S. 71. 
Laufe der Versuche innere und äußere Umstände durch unbestimmbare Zufälligkeiten. Die Zeit- und Raumlage selbst, der Einfluß der Herstellungsweise, die Aufmerksamkeit, der Zustand der Sinnesorgane unterliegen immer irgend welchen zufälligen Abänderungen, deren Spielraum größer oder geringer sein kann und Einfluß auf die durchschnittliche Größe der reinen Fehler gewinnt, wonach die daraus erhaltenen Summen oder Mittelwerte nur insofern zu einem vergleichbaren Maßstabe der Unterschiedsempfindlichkeit dienen können, als man voraussetzen darf, daß der Spielraum oder die durchschnittliche Größe der Zufälligkeiten in den verschiedenen Versuchen derselbe ist. Hätte aber z. B. die Aufmerksamkeit oder die Lage der verglichenen Größen während gewisser Versuche $a$ mehr geschwankt als während anderer Versuche $b$, so würde dies größere reine Fehler in $a$ als in $b$ mitführen, ohne daß deshalb die Unterschiedsempfindlichkeit in $a$ geringer als in $b \mathrm{zu}$ sein brauchte."

Während aber Fechner der Ansicht ist, daß durch diese Unsicherheit das Maß der Unterschiedsempfindlichkeit nur in einem gewissen, bei Wahrscheinlichkeitsbestimmungen unvermeidlichen Grade beeinträchtigt wird, findet G. E. Müller die Möglichkeit der Maßbestimmung völlig in Frage gestellt. Dabei ist allerdings die merkwürdige Auffassungsweise, die sich Müller über die Handhabung der Methode der mittleren Fehler gebildet hat, in Betracht zu ziehen. Er nimmt nämlich an, daß die Herstellung der Fehlreize und demgemäß der Betrag des mittleren Fehlers 1) "erstens davon abhängt, mit welchen relativen Häufigkeiten sich die Versuchsperson die verschiedenen Werte des Fehlreizes zur Vergleichung mit dem Normalreize $N$ herstellt, und zweitens davon, welche Wahrscheinlichkeit jeder Wert des Fehlreizes besitzt, im Falle seines Hergestelltwerdens zugelassen zu werden, d. h. einen hinlänglichen Schein der Gleichheit zu $N$ zu erwecken und demgemäß zur Notierung zu gelangen". Es soll hiernach möglich sein, daß die tatsächlich hergestellten Fehlreize sich enge um einen Wert scharen, „während andere von diesem Werte mehr abweichende Feblreize, welche einen hinlänglichen Anschein der Gleichheit zu $N$ gleichfalls erwecken können, überhaupt fast gar nicht hergestellt werden ".

1) Die Gesichtspunkte und die Tatsachen der psychophysischen Methodik 1904, S. 211. 
Wäre dies richtig, so wäre die Photometrie als Wissenschaft unmöglich; denn sie beruht auf der Möglichkeit, eine variierbare Helligkeit einer anderen gleich zu machen, ohne daß außer der Fähigkeit des Menschen, Helligkeiten zu unterscheiden und objektive Lichtstärken bis zu dem Punkte zu variieren, wo die Gleichheit mit größter Sicherheit erreicht scheint, etwas anderes in Betracht käme. Ebensowenig könnte der Violinspieler seine Violine stimmen. Denn er wäre von den relativen Häufigkeiten abhängig, mit denen er die verschiedenen Spannungen der Saite herstellt, während er doch aus der ganzen Reihe möglicher Spannungsstärken diejenige herzustellen glaubt, die den gewünschten Ton nach seinem Gehör am besten wiedergibt. Es wird aber jeder, der gleiche Helligkeiten herstellt oder eine Saite nach einem gegebenen Ton stimmt, es bestätigt finden, daß man bei der Variierung der Reize eine Stelle findet, wo der Vergleich des Feblreizes mit dem Normalreize am befriedigensten ausfällt und somit die Gleichheit mit größter Sicherheit erreicht ist. Aber auch dann, wenn nicht ein einzelner Wert, sondern ein ganzes Intervall gleichberechtigter Werte sich ergäbe, würde die Methode der mittleren Fehler nicht unmöglich gemacht, ja nicht einmal wesentlich erschwert werden. Man müßte nur die Intervallmitte zugleich mit der Intervalllänge notieren und bei der Verwertung der Versuche in Rechnung stellen.

Wenn aber auch demgemäß das Versuchsverfahren, das bei der Methode der mittleren Fehler zur Verwendung kommt, keinem Bedenken unterliegt, sondern das beste und sicherste ist, falls die Reize ungehindert und willkürlich verändert werden können, so bleibt doch der Mangel bestehen, daß, wie Fechner zugestanden und Müller mit Recht betont hat, der mittlere Fehler nicht bloß durch die Unterschiedsempfindlichkeit bedingt ist. Da die letztere in der Unterschiedsschwelle ihren unmittelbaren Ausdruck findet, so kann man somit nicht erwarten, daß der mittlere Fehler ausschließlich von der Unterschiedsschwelle abhängig sei. 'Dies wird durch die Bemerkung von Ebbinghau ${ }^{1}$ ) bestätigt, daß bei dem Versuchsverfahren zur Bestimmung des mittleren Fehlers und der Unterschiedsschwelle eine Erschwerung der Beurteilung durch Nötigung zu rascherer Urteilsfällung einesteils und durch

1) Grundzüge der Psychologie 1, 69 (1902). 
zeitliche Trennung des Vergleichsreizes vom Normalreize andererseits einmal der mittlere Fehler stärker als die Unterschiedsschwelle und das andere Mal die Unterschiedsschwelle stärker als der mittlere Fehler wächst. Hierdurch wird aber nur deutlich gemacht, daß die Methode der mittleren Fehler in der von Fechner gegebenen Form nicht hinreicht, sondern einer weiteren Ausbildung bedarf.

In dieser Erwägung habe ich in dem kleinen Grundriß der Psychophysik (1899) vorausgesetzt, daß die Wahrscheinlichkeit eines Fehlers $\Delta$ bei einer unendlich kleinen Unterschiedsschwelle durch das gewöhnliche Fehlergesetz

$$
\varphi(\Delta)=\frac{h}{\sqrt{\pi}} \exp \left(-h^{2} \Delta^{2}\right) . \quad . \quad . \quad .
$$

ausgedrückt und mithin unter der Herrschaft einer nach oben und nach unten um den Betrag $i$ abweichenden Unterschiedsschwelle durch

$$
2 i \cdot W(\Delta)=\frac{1}{2} \Phi[h(\Delta+i)]-\frac{1}{2} \Phi[h(\Delta-i)] .
$$

bezeichnet werde, wo

$$
\Phi[h \Delta]=\frac{2}{\sqrt{\pi}} \int_{0}^{h \Delta} \exp \left(-h^{2} \Delta^{2}\right) \cdot d(h \Delta) . .
$$

Dann ist, wenn

$$
\begin{aligned}
& m \varepsilon_{1}=\Sigma|\Delta|=\left|\Delta_{1}\right|+\left|\Delta_{2}\right|+\cdots+\left|\Delta_{m}\right| \mid \\
& \left.m \varepsilon_{2}^{2}=\Sigma \Delta^{2}=\Delta_{1}^{2}+\Delta_{2}^{2}+\cdots+\Delta_{m}^{2}\right\}
\end{aligned} .
$$

gesetzt wird, bei einer hinreichend großen Anzahl von Beobachtungen

$$
\left.\begin{array}{l}
\varepsilon_{1}=\frac{1}{m} \Sigma|\Delta|=2 \int_{0}^{\infty} \Delta \cdot W(\Delta) \cdot d \Delta \\
\varepsilon_{2}^{2}=\frac{1}{m} \Sigma \Delta^{2}=2 \int_{0}^{\infty} \Delta^{2} \cdot W(\Delta) \cdot d \Delta
\end{array}\right\}
$$

Es ist nun, wenn $h \Delta$ durch $\tau, h i$ durch $t$ und die successiven Ableitungen von $\Phi(\tau)$ durch $\Phi_{1}, \Phi_{2}, \Phi_{3} \ldots$ bezeichnet werden,

$$
W(\Delta)=\frac{\hbar}{2}\left\{\Phi_{1}(\tau)+\frac{t_{0}^{2}}{3 !} \Phi_{3}(\tau)+\frac{t_{0}^{4}}{5 !} \Phi_{j}(\tau)+\cdots\right\},
$$


so daß man durch Ausführung der Integrationen ohne weiteres

$$
\begin{gathered}
2 \int_{0}^{\infty} \Delta W(\Delta) d \Delta \\
\left.=\frac{1}{h \sqrt{\pi}}+\frac{i}{\sqrt{\pi}}\left\{\frac{2 t}{3.2 .1}-\frac{2 t^{3}}{5.4 .3 .1 !}+\frac{2 t^{5}}{7.6 .5 .7 !}-\cdots\right)\right\} \\
2 \int_{0}^{\infty} \Delta^{2} W(\Delta) d \Delta=\frac{1}{2 h^{2}}+\frac{i^{2}}{3}
\end{gathered}
$$

erhält. Um nun noch die unendliche Reihe

$$
a=\frac{2 t}{3.2 .1}-\frac{2 t^{3}}{5.4 .3 .1 !}+\frac{2 t^{5}}{7.6 .5 .3 !}-\cdots
$$

in eine bequemere Form zu bringen, beachte man, daß

$$
\frac{1}{2 v+1}-\frac{1}{v}+\frac{1}{2 v-1}=\frac{2}{(2 v+1) \cdot 2 v \cdot(2 v-1)}
$$

wonach die Zerlegung

$$
\begin{aligned}
& a=a_{1}+a_{2}+a_{3}, \\
& a_{1}=\frac{t}{3}-\frac{t^{3}}{5.1 !}+\frac{t^{5}}{7.2 !}-\cdots, \\
& a_{2}=-\frac{t}{1}+\frac{t^{3}}{2 !}-\frac{t^{5}}{3 !}+\cdots, \\
& a_{3}=\frac{t}{1}-\frac{t^{3}}{3.1 !}+\frac{t^{5}}{5.2 !}-\cdots,
\end{aligned}
$$

möglich ist. Es ist aber

$$
\begin{gathered}
a_{1}=\frac{1}{t^{2}} \int_{0}^{t} \tau^{2} \cdot \exp \left(-\tau^{2}\right) d \tau=\frac{1}{2 t^{2}} \int_{0}^{t} \exp \left(-\tau^{2}\right) d \tau-\frac{t}{2} \exp \left(-t^{2}\right) \\
a_{2}=\frac{1}{t}\left\{\exp \left(-t^{2}\right)-1\right\} \\
a_{3}=\int_{0}^{t} \exp \left(-\tau^{2}\right) d \tau
\end{gathered}
$$


so dals

$$
a=\left(\frac{1}{2 t^{2}}+1\right) \int_{0}^{t} \exp \left(-\tau^{2}\right) d \tau+\frac{1}{2 t}\left\{\exp \left(-t^{2}\right)-2\right\}
$$

resultiert. Man erbält so schließlich

$$
\left.\begin{array}{l}
2 \int_{0}^{\infty} \Delta W(\Delta) d \Delta=\frac{1}{h \sqrt{\pi}}+\frac{i}{2} A \\
2 \int_{0}^{\infty} \Delta^{2} W(\Delta) d \Delta=\frac{1}{2 h^{2}}+\frac{i^{2}}{3}
\end{array}\right\}
$$

wo zur Abkürzung

$$
A=\left(\frac{1}{2 t^{2}}+1\right) \Phi(t)+\frac{1}{t \sqrt{\pi}}\left\{\exp \left(-t^{2}\right)-2\right\}
$$

gesetzt wird.

Man hat nun zur Bestimmung des Unterschiedsschwellenwertes $i$ und des Präzisionsmaßes $h$ des Feblergesetzes auf Grund von (27) und (28) im Hinblick auf (29) und (30) die beiden Gleichungen

$$
\left.\begin{array}{l}
\varepsilon_{1}=\frac{1}{h \sqrt{\pi}}+\frac{i}{2} A \\
\varepsilon_{2}^{2}=\frac{1}{2 h^{2}}+\frac{i^{2}}{3}
\end{array}\right\}
$$

Hieraus kann man zunächst $t==h i$ finden, da

$$
\frac{\varepsilon_{1} \sqrt{\pi}}{\varepsilon_{2} \sqrt{2}}=\frac{1+1 / 2 t A \sqrt{\pi}}{\sqrt{1+2 / 3 t^{2}}}=\varphi(t) \ldots . .
$$

Hat man nämlich den Quotienten $\varphi(t)=\left(\varepsilon_{1} \sqrt{\pi}\right):\left(\varepsilon_{2} \sqrt{2}\right)$ aus den absoluten Beträgen und den Quadraten der $\Delta$ berechnet, so erbält man $t$ auf Grund der Tabelle 4, die einesteils die Werte $A$ und anderenteils die Werte $\varphi(t)$ in Abhängigkeit von $t$ gibt und nötigenfalls obne viele Mühe auf engere Intervalle der $t$ ausgedehnt werden kann.

Lipps, Die psychischen Mramethorlen. 
Tabelle 4.

\begin{tabular}{c|c}
\hline$t$ & $A$ \\
\hline 0,0 & 0,0000 \\
0,5 & 0,1835 \\
1,0 & 0,3432 \\
1,5 & 0,4682 \\
2,0 & 0,5607 \\
2,5 & 0,6286 \\
3,0 & 0,6794 \\
4,0 & 0,7492 \\
5,0 & 0,7943 \\
$\infty$ & 1,0000 \\
&
\end{tabular}

\begin{tabular}{c|c}
\hline$t$ & $\varphi(t)$ \\
\hline 0,0 & 1,000 \\
0,5 & 1,001 \\
1,0 & 1,010 \\
1,5 & 1,026 \\
2,0 & 1,041 \\
2,5 & 1,053 \\
3,0 & 1,061 \\
4,0 & 1,070 \\
5,0 & 1,075 \\
$\infty$ & 1,085 \\
&
\end{tabular}

Ist aber $t$ gefunden, so ergibt sich

$$
i=\frac{2 \varepsilon_{1} \sqrt{\pi} t}{2+t A \sqrt{\pi}} ; \quad h=\frac{2+t A \sqrt{\pi}}{2 \varepsilon_{1} \sqrt{\pi}} . . .
$$

wo nun $i$ als das reine MaB der in der Unterschiedsschwelle hervortretenden Unterschiedsempfindlichkeit und $h$ als ein von der Unterschiedsschwelle unabhängiges Maß der sonstigen, die Genauigkeit der Beobachtung störenden Einflüsse in Anspruch zu nehmen ist.

Die Annahme, daß die Häufigkeit des Auftretens der Fehler $\Delta$ in Übereinstimmung mit dem Fehlergesetze (22) geregelt werde, hat die Gleichung (33) zur Folge, wonach

$$
1<\frac{\varepsilon_{1} \sqrt{\pi}}{\varepsilon_{2} \sqrt{2}}<1,085 \text {. . . . . . . }
$$

sein muß. Ist diese Bedingung erfüllt, so kann $i$ und $h$ berechnet werden.

Nun hat Fechner bei Augenmaßversuchen ${ }^{1}$ ) aus $m=240$ Beobachtungen für die Distanzen 10, 20, 30, 40, 50 der Reihe nach

$$
\begin{aligned}
& \Sigma|\Delta|=38,64 ; 76,85 ; 120,91 ; 154,48 ; 185,40 \\
& \Sigma \Delta^{2}=8,677 ; 40,42 ; 97,67 ; 146,15 ; 228,46
\end{aligned}
$$

1) Elemente der Psychophysik 1, 215. 
gefunden, wo $1_{/ 2}^{1 / 2}$ Pariser Decimallinie als Einheit gewählt war. Es ist demgemäß der Reihe nach

$$
\frac{\varepsilon \sqrt{\pi}}{q \sqrt{2}}=1,061 ; 0,978 ; 0,990 ; 1,034 ; 0,992 ;
$$

so daß nur im ersten und im vierten Falle eine Bestimmung von $i$ und $h$ möglich ist. Man findet im ersten Falle $t=3,0$; $i=0,3 ; h=10 ; \mathrm{im}$ vierten Falle $t=1,75 ; i=1,1 ; h=1,6$. Da aber in den übrigen Fällen der Quotient kleiner als 1 ist, so zeigt sich, daß die Bedingung (35) und demgemä $\beta$ auch das Fehlergesetz (24) nicht zutrifft.

Man darf somit bei der Aufstellung eines das Vorhandensein von Unterschiedsschwellen berücksichtigenden Fehlergesetzes nicht von der Voraussetzung ausgehen, daß für den Fall einer unendlich kleinen Unterschiedsschwelle das gewöhnliche Fehlergesetz gelte. Man muls vielmehr eine Ausgestaltung der Methode der mittleren Fehler erstreben, die von der Annahme eines bestimmten Fehlergesetzes völlig absieht.

\section{Die Methode der richtigen und falschen Fälle.}

Sind die Reizwerte nicht während ihrer Einwirkung auf das Sinnesorgan beliebig und willkürlich variierbar, so kann nur ein bestimmt gewählter Reiz mit einem benachbarten, gleichfalls bestimmt gewählten Reize immer und immer wieder verglichen werden. Dies Verfahren führt zur Methode der richtigen und falschen Fälle. Hat man nun beispielsweise ein Hauptgewicht $P$ mit einem durch ein kleines Zusatzgewicht $D$ vergrößerten Vergleichsgewichte $P+D$ im ganzen $m$ mal verglichen, und haben sich $p$ Urteile "größer", $z$ Urteile "gleich" und $n$ Urteile "kleiner" ergeben, so weist Fechner von den $z$ Urteilen die eine Hälfte den Urteilen "größer", die andere Hälfte den Urteilen „kleiner" zu und bezeichnet die $r=p+1 / 2 z$ Urteile als nrichtige", die $f=n+1 / 2 z$ Urteile als "falsche".

Die relativen Häufigkeits- oder Wahrscheinlichkeitswerte

$$
\frac{r}{m}=\frac{p+1 / 2 z}{m} ; \frac{f}{m}=\frac{n+1 / 2 z}{m} . .
$$


sind von der Unterschiedsempfindlichkeit abhängig. Sie ändern. sich aber auch bei gleicher Unterschiedsempfindlichkeit mit den Zusatzgewichten und mit sonstigen, konstante Fehler bedingenden Umständen, die man der Einfachleit wegen durch eine Vermehrung oder Verminderung des Zusatzgewichtes ersetzt denken kann. Das Maß der Unterschiedsempfindlichleit bezeichnet Fechner durch $h$, das $M a B$ des Zusatzgewichtes und der sonstigen konstanten Einflüsse durch $A$. Er nimmt ferner an, daß die Wahrscheinlichkeitswerte $r / m$ und $f / m$ von dem Produkte $h A$ oder, wenn keine sonstigen Einflüsse in Betracht kommen, von $h D$ abhängen, und daß die Abhängigkeit zwischen $r / m$ einerseits und dem durch $t$ bezeichneten Produkte $h A$ andererseits durch das Gausssche Feblergesetz geregelt werde, wonach die in der Tabelle 5 verzeichneten Werte $r / m$ und $t$ einander entsprechen.

\section{Tabelle 5 .}

Fundamentaltabelle der Methode der richtigen und falschen Fälle.

\begin{tabular}{c|c|c|c|c|c}
\hline$\frac{r}{m}$ & $t=h D$ & $\frac{r}{m}$ & $t=h D$ & $\frac{r}{m}$ & $t=h D$ \\
\hline 0,50 & 0,0000 & 0,67 & 0,3111 & 0,84 & 0,7032 \\
0,51 & 0,0177 & 0,68 & 0,3307 & 0,85 & 0,7329 \\
0,52 & 0,0355 & 0,69 & 0,3506 & 0,86 & 0,7639 \\
0,53 & 0,0532 & 0,70 & 0,3708 & 0,87 & 0,7965 \\
0,54 & 0,0710 & 0,71 & 0,3913 & 0,88 & 0,8308 \\
0,55 & 0,0890 & 0,72 & 0,4121 & 0,89 & 0,8673 \\
0,56 & 0,1068 & 0,73 & 0,4333 & 0,90 & 0,9062 \\
0,57 & 0,1247 & 0,74 & 0,4549 & 0,91 & 0,9481 \\
0,58 & 0,1428 & 0,75 & 0,4769 & 0,92 & 0,9936 \\
0,59 & 0,1609 & 0,76 & 0,4994 & 0,93 & 1,0436 \\
0,60 & 0,1791 & 0,77 & 0,5224 & 0,94 & 1,0994 \\
0,61 & 0,1974 & 0,78 & 0,5460 & 0,95 & 1,1631 \\
0,62 & 0,2160 & 0,79 & 0,5702 & 0,96 & 1,2379 \\
0,63 & 0,2347 & 0,80 & 0,5951 & 0,97 & 1,3297 \\
0,64 & 0,2535 & 0,81 & 0,6208 & 0,98 & 1,4531 \\
0,65 & 0,2725 & 0,82 & 0,6473 & 0,99 & 1,6438 \\
0,66 & 0,2917 & 0,83 & 0,6747 & 1,00 & $\infty$
\end{tabular}


In dieser Tabelle findet man zu dem beobachteten Werte $r / m$ das zugehörige $t$ und hieraus bei Abwesenheit konstanter Fehler das Präzisionsma $h$, das die Unterschiedsempfindlichkeit mißt. Haben sich z. B. für das Hauptgewicht $P=300 \mathrm{~g}$ und das Vergleichsgewicht $P+D=312 \mathrm{~g}$ aus 512 Versuchen $r=328$ richtige Fälle ergeben, so daß $r / m=0,641$, so findet man aus der Fundamentaltabelle $t=0,255$ und, da $D=12 \mathrm{~g}$, $h=0,021$.

In Wahrheit treten aber konstante Fehler auf. Die scheinbare Schwere eines Gewichtes ändert sich nämlich, wie Fechner gefunden hat, je nachdem es an erster oder zweiter Stelle gehoben wird, indem wenigstens bei größeren Gewichten das an zweiter Stelle gehobene durchschnittlich schwerer erscheint, so daß hierdurch ein nicht unbedeutendes Zusatzgewicht bei dem zuerst gehobenen kompensiert werden kann. Es kommt ferner in Betracht, ob man mit der rechten oder mit der linken Hand die Hebung ausführt, und was für eine Art und Weise des Hebens durch die jeweilige Lage des Gewichtes bezüglich der die Hebung ausführenden Hand bedingt wird. Diese Einflüsse wirken nach der Annahme Fechners wie ein zu dem vorhandenen Zusatzgewichte $D$ hinzutretendes positives oder negatives neues Zusatzgewicht $\pm p$, so da $A=D \pm p$ ist. Insbesondere setzt Fechner voraus, daß der Einfluß der Zeitlage bei einer bestimmten Aufeinanderfolge von Hauptgewicht und Vergleichsgewicht durch. $D+p$, bei der entgegengesetzten Aufeinanderfolge durch $D-p$ zum Ausdruck gebracht werde, so daß, wenn im ersten Falle $t_{1}$, im zweiten Falle $t_{2}$ aus den Versuchen gefunden worden ist, aus

$$
h(D+p)=t_{1} ; h(D-p)=t_{2}
$$

die Gleichungen

$$
h D=1 / 2\left(t_{1}+t_{2}\right) ; h p=1 / 2\left(t_{1}-t_{2}\right) . .
$$

sich ergeben. In ähnlicher Weise werden auch die aus der Raumlage entspringenden konstanten Febler eliminiert und ihrer Größe nach bestimmt.

Indem Fechner das angegebene Verfahren aus unzulänglichen, in den Versuchen einiger Schüler des Physiologen Vierordt vorliegenden Anfängen entwickelte, setzte er zwar das gewöhnliche Feblergesetz (24) bei der Herleitung der Funda- 
mentaltabelle (Tabelle 5) voraus; er ließ aber die hieraus sich ergebenden Folgerungen nicht in ihrem vollen Umfange zur Geltung kommen. Die Annahme eines Fehlergesetzes hat nämlich zur Folge, daß bei der Vergleichung der Gewichte $P$ und $P+D$ ein Fehler $\Delta$ mit der durch das Gesetz bedingten Häufigkeit begangen wird. Wodurch die Fehler veranlaßt werden, bleibt hierbei völlig dahin gestellt. Sie können aus der einen oder der anderen von dell drei Fehlerquellen, die schon Lambert (S. 35) unterschieden hat, herrühren. Deuten wir aber die Gewichte $P$ und $P+D$ als Punkte einer Geraden, so können wir uns stets den Erfolg des Fehlervorganges dadurch veranschaulichen, daß wir uns den Punkt $P+D$ als festliegend und den Punkt $P$ um den Betrag $\Delta$ nach der einen oder der anderen Seite verschoben denken, so daß an Stelle der wahren Differenz $D$ die scheinbare Differenz $D+\Delta$ oder $D-\Delta$ tritt. Die Annahme, daß sowohl $P$ als auch $P+D$ durch die Fehlervorgänge ihre Lage ändern, ist entbehrlich; sie führt bloß zu überflüssigen Umständlichkeiten.

Auf Grund des gewöhnlichen Fehlergesetzes (24) wird nun ein zwischen den unendlich nahen Grenzen $\Delta$ und $\Delta+d \Delta$ liegender Fehler mit der relativen Häufigkeit $\varphi(\Delta) d \Delta$ begangen. Es müßte somit, wenn keine Unterschiedsschwelle in Betracht käme, $P$ entweder kleiner als $P+D$ oder größer als $P+D$ erscheinen, so oft der Punkt $P$ in das Gebiet unterhalb $P+D$ oder oberhalb $P+D$ fiele. Die relative Häufigkeit der richtigen und der falschen Urteile würde daher durch

$$
\left.\begin{array}{c}
\frac{r}{m}=\int_{-D}^{\infty} \varphi(\Delta) d \Delta=\int_{0}^{\infty} \varphi(\Delta) d \Delta+\int_{0}^{D} \varphi(\Delta) d \Delta \\
=1 / 2+1 / 2 \Phi(h D), \\
\frac{f}{m}=\int_{-\infty}^{\infty} \varphi(\Delta) d \Delta=\int_{0}^{\infty} \varphi(\Delta) d \Delta-\int_{0}^{D} \varphi(\Delta) d \Delta \\
=1 / 2-1 / 2 \Phi(h D)
\end{array}\right\}
$$

bestimmt, wo $\Phi$ die in (24) angegebene Bedeutung hat, während die relative Häufigkeit der scheinbaren Gleichheit von $P$ mit $P+D$ gleich Null zu setzen wäre. Diese Bestimmungen finden 
in der Fundamentaltabelle Fechners ihren Ausdruck. Da aber eine Unterschiedsschwelle vorbanden ist, so treten neben deu Urteilen "größer" und "kleiner" auch die von Fechner ohne Rücksicht auf das Fehlergesetz in der oben angegebenen Weise beseitigten Urteile "gleich" auf. Hat die Unterschiedsschwelle in der Richtung der wachsenden und der abnehmenden Werte, wie wir der Einfachheit wegen annehmen wollen, einen und denselben Betrag $i$, so sind demgemä $B$ die relativen Häufigkeiten der Urteile ${ }_{n} P+D$ ist größer als $P^{u}$, ${ }_{n}$ gleich $P^{4}$, ${ }_{n}$ kleiner als $P^{4}$ der Reihe nach gleich

$$
\begin{gathered}
\frac{p}{m}=\int_{-D+i}^{\infty} \varphi(\Delta) d \Delta=\int_{0}^{\infty} \varphi(\Delta) d \Delta+\int_{0}^{D-i} \varphi(\Delta) d \Delta \\
=1 / 2+1 / 2 \Phi[h(D-i)] ; \\
\frac{z}{m}=\int_{-D-i}^{D+i} \varphi(\Delta) d \Delta=\int_{0}^{D+i} \varphi(\Delta) d \Delta-\int_{0}^{D-i} \varphi(\Delta) d \Delta \mid \\
=1 / 2 \Phi[h(D+i)]-1 / 2 \Phi[h(D-i)] ; \\
\frac{n}{m}=\int_{-\infty}^{D-i} \varphi(\Delta) d \Delta=\int_{0}^{\infty} \varphi(\Delta) d \Delta-\int_{0}^{\infty} \varphi(\Delta) d \Delta \\
=1 / 2-1 / 2 \Phi[h(D+i)] .
\end{gathered}
$$

Diese Festsetzungen werden durch Fig. 3 veranschaulicht, wo $P+D$ durch $P_{1}, P+D+i$ durch $P_{2}$ und $P+D-i$

Fig. 3.

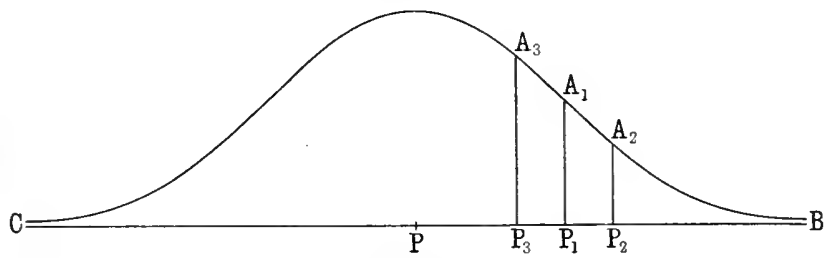

durch $P_{3}$ bezeichnet wird, so daß $P_{2}$ und $P_{3}$ die zu $P_{1}$ gehörende obere und untere Unterschiedsschwelle markieren. Es ist nämlich für die subjektive Auffassung $P+D$ größer als $P$, gleich $P$ oder kleiner als $P$, so oft der Fehlervorgang den Punkt $P$ in dem Gebiete unterhalb $P_{3}$ beläßt, in das Gebiet zwischen $P_{3}$ und $P_{2}$ oder 
in das Gebiet oberhalb $P_{2}$ verlegt, so daß die relativen Häufigkeiten $p / m, z / m, n / m$ der Reihe nach gleich den Flächenstücken $C A_{3} P_{3}, A_{3} P_{3} P_{2} A_{2}, A_{2} P_{2} B$ sind. Die relativen Häufigkeiten $v / m$ und $f / m$ hingegen werden durch die Flächenstücke $C P_{1} A_{1}$ und $B P_{1} A_{1}$ dargestellt.

Diese auf der Berücksichtigung der Unterschiedsschwelle beruhende Weiterbildung der Methode der richtigen und falschen Fälle hat G. E. Müller in seiner "Grundlegung der Psychophysik“. (1879) gegeben. Er gelangte so dazu, neben dem Präzisionsmaße auch die Unterschiedsschwellen aus den beobachteten Häufigkeiten der Urteile "größer", "gleich" und „kleiner" zu berechnen.

Setzt man nämlich

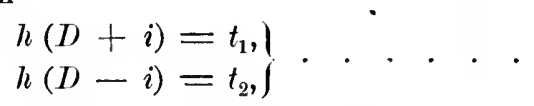

so findet man aus der Fundamentaltabelle zu dem Quotienten $p / m$ den Wert $t_{2}$ und zu dem Quotienten $(p+z): m$ den Wert $t_{1}$, so daß

$$
\left.\begin{array}{l}
l=\frac{t_{1}+t_{2}}{2 D} \\
i=\frac{t_{1}-t_{2}}{t_{1}+t_{2}} D
\end{array}\right\} . . . .
$$

Man erhält so nicht nur wie bei dem ursprünglichen Verfahren Fechners in dem Präzisionsmaße $h$ einen von der Unterschiedsschwelle und auch von anderen Fehlerquellen abbängigen Wert, sondern zugleich in der Unterschiedsschwelle $i$ den unmittelbaren Maßstab der Unterschiedsempfindlichkeit. Es ließe sich zwar einwenden, daß $i$ dem Vergleichsreize $P+D$ und nicht dem Hauptreize $P$ zugehört. Indessen darf $i$ innerhalb des durch die Differenz $D$ bestimmten Bereiches als konstant betrachtet werden. Es ist nämlich zu beachten, daß die Bestimmung von $i$ durch die Schwankungen und Störungen, mit denen die empirisch gefundenen Häufigkeitswerte $p, z, n$ behaftet sind, beeinträchtigt wird. Darum kann der jeweils sich ergebende Wert kein unbedingtes Zutrauen beanspruchen, sondern nur als Repräsentant einer ganzen Mannig-· faltigkeit von Werten, die bei unbegrenzter Wiederholung der Beobachtungen hervortreten würden, zur Geltung kommen. Die demgemä $ß$ in Rechnung zu ziehenden Unregelmäßigkeiten werden 
aber in der Regel bedeutender sein als die Differenzen der normalen Unterschiedsschwellenwerte, die sich etwa dem Weberschen Gesetze zufolge beim Übergange von $P$ zu $P+D$ einstellen. Darum darf es auch nicht befremden, wenn bei einer Prüfung der Zuverlässigkeit, mit der $h$ und $i$ im Vergleich miteinander als $\mathrm{MaB}$ der Unterschiedsempfindlicheit sich bewähren, gelegentlich die Werte von $i$ weniger brauchbar als die Werte von $h$ sich erwiesen haben. Es muß darum doch $i$ als das reinere, wenn auch je nach Umständen von den Unregelmäßigkeiten der empirisch bestimmten Häufigkeiten stärker in Mitleidenschaft gezogene $\mathrm{Ma}$ der Unterschiedsempfindlichkeit festgehalten werden.

Ungenauigkeiten der Werte $i$ und $h$ sind ja von vol'nherein anzunehmen. Denn eine immer wieder ausgeführte Wiederholung der Beobachtungen würde zu anderen und anderen Wahrscheinlichkeitswerten $p / m, z / m, n / m$ und dementsprechend auch zu verschiedenen Werten $i$ und $h$ führen, so daß, wie es bei induktiven Wahrscheinlichkeitsbestimmungen nicht anders sein kann, die tatsächlich gefundenen Werte $i$ und $h$ als Repräsentanten von je einer ganzen Mannigfaltigkeit von Werten sich darbieten. Es müßte darum noch die mittlere Abweichung der Einzelwerte von ihrem arithmetischen Mittel auf Grund des vorausgesetzten Fehlergesetzes berechnet und zur Charakterisierung der repräsentierten Mannigfaltigkeiten den Werten $i$ und $h$ in (41) binzugefügt werden, wenn die Bestimmungen vollständig sein sollten $\left.{ }^{1}\right)$.

Treten überdies konstante Fehler auf, so kann bierdurch, eben weil das Fehlergesetz vorausgesetzt wird, bloß die Verschiebung des Punktes $P$ um den Betrag des konstanten Fehlers bewirkt werden. Die Gestalt der Kurve samt der gegenseitigen Lage der Punkte $P_{1}, P_{2}, P_{3}$, in Fig. 3 bleibt hingegen erhalten, da der

1) Wegen dieser Unbestimmtheit wird man sich überhaupt nicht mit einer einzigen Differenz $D$ begnügen, sondern eine ganze Reihe von Differenzen unter übereinstimmenden Umständen beobachten. Man erhält alsdann an Stelle von (39) ein System solcher Gleichungen, aus dem die Unbekannten, da mehr Gleichungen als Unbekannte vorhanden sind, durch ein Ausgleichungsverfahren zu bestimmen sind. Daß hierbei die Methode der kleinsten Quadrate anwendbar ist, hat Bruns in der Abhandlung, „Über die Ausgleichung statistischer Zählungen in der Psychophysik" durch eine von den Grundsätzen der Wahrscheinlichkeitslehre ausgehende Untersuchung festgestellt. 


\section{- $74-$}

hierfür maßgebende Parameter $h$ und Unterschiedsschwellenwert $i$ der Definition gemäß von dem konstanten Fehler unabbängig sind. Es kann somit weder eine flachere noch eine steilere Form auftreten, wie dies beim Übergang von der gestrichelten zur ausgezogenen Kurve oder von der letzteren zur ersteren in Fig. 2 (S. 38) der Fall ist. Darum ist die Annahme Fechners, daß die konstanten Fehler wie ein zum Zusatzgewichte $D$ hinzutretendes positives oder negatives neues Zusatzgewicht $\pm p$ wirken, durchaus im Einklang mit der Voraussetzung des Fehlergesetzes. Ja, es köunte geradezu eine Bestätigung der Geltung des Fehlergesetzes darin gefunden werden, wenn in der Tat nicht nur der Fehler der Zeitlage, sondern auch alle sonstigen Einflüsse nach dem durch Fechner angegebenen Verfahren eliminiert und bestimmt werden köunten.

Es lassen sich aber nicht alle Besonderheiten der Beobachtungsweise nach dem Fechnerschen Schema behandeln. In dieser Hinsicht sind namentlich die Untersuchungen von Lillie Martin und G. E. Müller, „Zur Analyse der Unterschiedsempfindlichkeit" (1891) zu beachten. Sie finden ibren theoretischen Stützpunkt in der Lehre vom absoluten Eindrucke ${ }^{1}$ ), worunter der Eindruck der Leichtigkeit oder der Schwere verstanden wird, „den ein gehobenes Gewicht, isoliert genommen, d. h. ohne Vergleichung mit einem bestimmten vor oder nach ihm gehobenen Gewichte, macht". Der absolute Eindruck ist somit offenbar auf die Beeinflussung durch frühere Erlebnisse zurückzuführen, die wir in $\S 2$ (S. 14) als eine besondere Quelle von Bedingungen, denen die Bewußtseinsinhalte unterliegen, hervorgehoben haben. Dies bestätigt sich durch die bei Martin und Müller unmittelbar sich anschließende Erläuterung. „Wie uns ein Gegenstand des gewöhnlichen Lebens, ein Brief, ein Buch, ein Koffer u. dgl. oder z. B. auch ein Kind beim Heben schwer oder leicht erscheinen kann, ohne daß wir hierbei diesen Gegenstand mit einem bestimmten anderen Gegenstande derselben Art vergleichen, so kann auch bei Versuchen mit gehobenen $\mathrm{Ge}_{\theta}$ wichten uns ein Gewicht schwer oder leicht erscheinen, ohne daß es hierbei mit einem bestimmten anderen Gewichte verglichen wird." Durch diesen absoluten Eindruck finden besondere Ur-

1) „Zur Analyse der Unterschiedsempfindlichkeit", S. 43. 
teilssentenzen und typische Verhaltungsweisen der Beobachter ihre Erklärung, die zu „anomalen Differenzen der erhaltenen Urteilszahlen" führen.

Wichtiger als diese Erklärung ist aber für uns die Tatsache, daß es überhaupt Anomalien gibt, die sich dem Fechnerschen Schema nicht fügen. Sie liefern den Beweis, daß es nicht gestattét ist, die Methode der richtigen und falschen Fälle auf die Annahme, daß in jedem Falledas gewöhnliche Fehlergesetz Geltung habe, zu gründen.

Dies bestätigt die Erkenntnis, die wir bezüglich der Methode der mittleren Fehler bereits gewonnen haben. Wenn nämlich bei Anwendung der Methode der richtigen und falschen Fälle die Wahrscheinlichkeit dafür, daß ein gegebener Reiz $P$ dem ebenfalls gegebenen Reize $P+D$ gleich erscheine, in Übereinstimmung mit (39) in der Form

$$
1 / 2 \Phi[h(D+i)]-1 / 2 \Phi[h(D-i)]
$$

voransgesetzt wird, so ist auch für die Wabrscheinlichkeit, daß bei der Methode der mittleren Fehler die Herstellung eines variierbaren, dem gegebenen $P$ anscheinend gleichen Reizes der Reiz $P+D$ getroffen wird, in Übereinstimmung mit (25) der Wert

$$
W(D)=\frac{1}{4 i} \Phi[h(D+i)]-\frac{1}{4 i} \Phi[h(D-i)]
$$

anzunehmen. Denn so oft bei der Herstellung gleich beurteilter Reize ein zwischen den Grenzen $P+D-i$ und $P+D+i$ liegender Reiz getroffen wird, ebenso oft muß bei der Darbietung gegebener Reize der Reiz $P+D$ dem Reize $P$ gleich erscheinen. Die Differenz zwischen dem dargebotenen Werte $P+D$ und dem bei freier Verfügung über die Reizskala hergestellten Reizwerte ist ja kleiner als der Unterschiedsschwellenwert $i$ und kann somit das Auftreten des Urteils ${ } P+D$ ist gleich $P{ }^{*}$ nicht hindern. Nun nimmt allerdings die Wahrscheinlichkeit innerhalb der Grenzen $P+D-i$ und $P+D+i$ nicht gleichmäßig, sondern in Übereinstimmung mit dem Fehlergesetze ab. Man erhält jedoch eine durchaus hinreichende Annäherung, wenn man der Einfachheit wegen eine gleichmäßige Abnahme voraussetzt. Dann ist aber der zur Intervallmitte $P+D$ gehörende Wahrscheinlichkeitswert $W(D)$ zugleich der Mittelwert aller dem ganzen Inter- 
valle $P+D \pm i$ zugehörenden Wahrscheinlichkeitswerte, deren Summe gleich

$$
1 / 2 \Phi[h(D+i)]-1 / 2 \Phi[h(D-i)]
$$

ist Es ist folglich

$$
2 i W(D)=1 / 2 \Phi[h(D+i)]-1 / 2 \Phi[h(D-i)] \text {, }
$$

so daß in der Tat die Formeln (25) und (39) sich wechselweise bedingen und somit die Unzulänglichkeit der einen Formel zugleich die Unzulänglichkeit der anderen Formel zur Folge hat.

Man gewinnt so zugleich die Einsicht, daßzwischen der Methodeder mittleren Fehler und der Methodeder. richtigen und falschen Fälle ein fehlertheoretischer \%usammenhang besteht, indem für jedes beliebige, voraussetzungsweise oder tatsächlich geltende Fehlergesetz zu der Wahrscheinlichkeit $W(\Delta)$ eines bei der Herstellung gleich beurteilter Reize auftretenden Fehlers die Wahrscheinlichkeit $2 i W(\Delta)$ für die anscheinende Gleichheit zweier um den Betrag $\Delta$ verschiedener, zur Beurteilung dargebotener Reize gehört.

\section{Die Methode der mittleren Abstufungen.}

Im Hinblick auf die Schwierigkeiten, die durch das Versagen des gewöhnlichen Fehlergesetzes einer Anwendung der Methode der richtigen und falschen Fälle und der mittleren Fehler erwachsen, könnte es als das Einfachste und Sicherste erscheinen, das Wachsen der Empfindung in seiner Abhängigkeit vom Reize unmittelbar zu verfolgen, indem man drei übermerklich verschiedene Reizgrößen $x_{1}, x_{2}, x_{3}$ so zu bestimmen sucht, daß die zugebörigen Empfindungsintensitäten $y_{1}, y_{2}, y_{3}$ in gleichem Maße verschieden erscheinen. Wäre dies dann und nur dann der Fall, wenn $x_{1}: x_{2}=x_{2}: x_{3}$, so könnte man schließen, $\mathrm{da} ß \mathrm{zu}$ gleichen Verhältnissen der objektiven Reizstärken gleiche Unterschiede der subjektiven' Empfindungsintensitäten gehören, wonach der Formel (19) zufolge das psychophysische Grundgesetz unmittelbar als gültig sich erweisen würde und allgemein $y=c \cdot \log x$ zu setzen wäre.

Daß überhaupt die Möglichkeit besteht, eine Empfindung mit Bestimmtheit als die Mitte zwischen einer stärkeren und 
einer schwächeren Empfindung zu bezeichnen, bat zuerst Platea $u$ in einer 1872 veröffentlichten Abhandlung ${ }^{1)}$ nachgewiesen. Er machte darauf aufmerksam, daß wir einen Gegenstand als hellgrau bezeichnen, wenn sein Grau dem Weiß näher steht als dem Schwarz, so daß angeblich "die Stärke der in uns erregten Empfindung größer als die Hälfte derjenigen Empfindungsstärke ist, die ein weißer Gegenstand unter den nämlichen Beleuchtungsverhältnissen erregen würde. Wenn wir hingegen sagen, daß ein Gegenstand dunkelgrau ist, so verstehen wir darunter, daß sein Grau dem Schwarz näher steht als dem Weiß, oder mit anderen Worten, da $B$ die ihm entsprechende Empfindungsstärke kleiner ist als die Hälfte derjenigen, die einem weißen in gleicher Weise beleuchteten Gegenstande zugehört". Schießlich können wir uns, wie Platea u bemerkt, ein Grau verschaffen, das genau ebenso weit vom Weiß wie vom Schwarz entfernt erscheint und folglich eine Empfindung erregt, deren Intensität angeblich gleich der Hälfte der durch Weiß erzeugten Empfindungsstärke ist.

In der Tat ergab es sich, daß acht der Malerei kundige Personen, die von Plateau aufgefordert wurden, für reines Weiß und reines Schwarz das mittlere Grau herzustellen, zu fast völlig übereinstimmenden Ergebnissen gelangten.

Man kann hierbei ebenso wie bei der Methode der mittleren Fehler und bei dem von Fechner angegebenen Verfahren der Herstellung eben merklicher Unterschiede einen beliebig und willkürlich veränderlichen Rciz während seiner Einwirkung auf das Sinnesorgan so verändern, daß die zugehörige Empfindung von den beiden durch die konstanten Reize vermittelten Empfindungen in gleichem Maße verschieden erscheint. Man kann auch, wenn dies nicht möglich ist, einen passend gewählten mittleren Reiz neben den beiden anderen Reizen immer wieder darbieten und die Urteile zählen, wie oft der wittlere Reiz dem einen oder dem anderen Grenzreize näher oder in der Mitte zwischen beiden zu liegen scheint. Es handelt sich somit darum, eine mittlere Abstufung zwischen zwei gegebenen Reizen herzustellen oder eine gegebene mittlere Abstufung zu beurteilen, weshalb man das

1) Sur la formule de la loi psychophysique; Bulletins de l'Académie Royale de Belgique; 33 e année; 1872 , p. 376. 
Verfahren die Methode der mittleren Abstufungen genannt hat.

Will man aber auf dem einen oder dem anderen Wege die Nitte zwischen zwei Empfindungen bestimmen, so muß man doch wohl darüber klar sein, was normalerweise als die Mitte von Empfindungen zu gelten hat. Es ist ja das Erfassen und Unterscheiden einer mittleren Empfindung ebenso wie einer Empfindung überhaupt (nach $\S 2$ ) vierfach bedingt: durch die Beschaffenheit des Reizes; durch das gleichzeitig Wahrgenommene, also insbesondere durch die Empfindungen, welche die zu halbierende "Empfindungsstrecke" abgrenzen; durch das unmittelbar zuvor Wahrgenommene, von dem sich die mittlere Empfindung und die Grenzempfindungen abheben, und schließlich durch die Gesamtheit der im Beobachter angehäuften Erfahrungen. Nun scheint es mir unmittelbar einleuchtend zu sein, daß eine Empfindung $B$ nicht als die zu zwei anderen Empfindungen $A$ und $C$ gehörige Mitte erkannt werden kann, wenn man nicht die nach Ähnlichkeitsgraden abgestufte, von $A$ über $B$ nach $C$ führende Folge von Empfindungen erlebt hat. Darum können die drei zuerst genannten Bedingungen die Feststellung der mittleren Empfindung zwar stören, aber nicht von Haus aus bedingen. Die Norm für die Feststellung der Empfindungsmitte beruht vielmehr lediglich auf dem angehäuften Schatze von Erfahrungen, die eine gewisse Kenntnis der die Grenzempfindungen verknüpfenden Stufenfolge von Empfindungen ermöglichen, ohne daß jedoch hierbei eine Reproduktion dieser Stufenfolge im Bewußtsein erfolgt.

Wenn beispielsweise die Geruchsempfindungen in verschiedene Klassen (ätherische, aromatische, balsamische Gerüche usw.) eingeteilt werden, so wird hierbei stillschweigend vorausgesetzt, daß wenigstens innerhalb einer und derselben Klasse je zwei verschiedene Empfindungen durch stetige Übergänge verknüpft werden können. Denn wäre dies nicht der Fall, so wäre die Einteilung nicht berechtigt. Demnach sind zwischen zwei ätherischen Gerüchen (Apfel, Ananas) oder zwei aromatischen Gerüchen (Kampfer, Pfeffer) oder zwei balsamischen Gerüchen (Jasmin, Veilchen) stetige Übergänge und somit auch mittlere Empfindungen vorauszusetzen. Es wird sich aber wohl niemand imstande fühlen, von einer Geruchsempfindung, die zu der die beiden Grenzempfindungen verknüpfenden Reihe gehört, mit Be- 
stimmtheit anzugeben, ob sie die Mitte hält oder nach der einen oder der anderen Seite abweicht; es sei denn, daß er bereits Erfahrungen über die Geruchsreihen gesammelt hat und auf Grund dieser Erfahrungen ein Urteil fällen kann.

Dem widerstreitet die Übereinstimmung, welche die acht, der Malerei kundigen Personen nach Plateau bei der Herstellung des mittleren Grau erzielten, keineswegs; sie erklärt sich vielmehr gerade durch die Beschäftigung mit Malerei, wodurch eine übereinstimmende Kenntnis der Helligkeitsabstufungen und eine gleichmäßige Übung in ihrer Unterscheidung erzielt worden war. Überdies bietet schon jeder aufdämmernde Tag und jede hereinbrechende Nacht immer wieder die ganze Stufenfolge von Helligkeiten dar, so daß jedes Auge bereits durch die tägliche Erfahrung eine gewisse Kenntnis derselben erwirbt.

Die Bestimmung der Mitte zwischen zwei gegebenen Empfindungen ist sonach normalerweise durch beiderseits gleich grobe Auzahlen eben unterscheidbarer Zwischenstufen bedingt, die sich im Bewußtsein als übereinstimmende Gradeder Ähnlichkeit und des Gegensatzes zu erkennen geben. Daß aber diese Norm nicht allein in Betracht kommt, ist selbstverständlich. Wenn nämlich bei der Herstellung anscheinend gleicher oder eben unterscheidbarer Reize und bei der Beurteilung unter merklich oder eben merklich verschiedener Reize konstante Fehler von beträchtlicher Größe begangen werden, so sind bei der Herstellung oder der Beurteilung von Reizen, deren Empfindungen in der Mitte zwischen gegebenen Empfindungen liegen sollen, noch weit größere konstante Fohler zu erwarten. Entspricht insbesondere den beideu von der mittleren Empfindung aus nach beiden Seiten sich erstreckenden Reihen eben unterscheidbarer Zwischenstufen auf der einen Seite ein größeres Reizgebiet als auf der anderen, so kann durch die Anhäufung von hierauf sich beziehenden Erfahrungen eine Täuschung hinsichtlich der Empfindungsmitte bedingt werden. Man wird alsdann, wenn etwa die normale Mitte der Empfindúngsreile das geometrische Mittel der Grenzreize fordern würde, einen nach dem arithmetischen Mittel zu verschobenen Reizwert für die scheinbare Empfindungsmitte finden. Sind ferner gewisse Glieder der zusammenhängenden Reihe von Empfindungen, wie z. B. die den musikalischen Intervallen entsprechenden Glieder 
in der Reihe eben unterscheidbarer Töne, durch Besonderbeiten ausgezeichnet, so wird man geneigt sein, einer solchen ausgezeichneten, der wahren Mitte bloß angenäherten Empfindung die Rolle der mittleren Empfindung zuzuweisen. Es können überhaupt, wie man zusammenfassend sagen kann, Beziehungen der Ähnlichkeit und des Gegensatzes, die zwischen einer mittleren Empfindung und den beiden Grenzempfindungen bestehen, aber nicht ausschließlich durch die Zwischenstufen bedingt sind, das Urteil über die Empfindungsmitte beeinflussen.

Die Methode der mittleren Abstufungen führt nun zur Kenntnis desjenigen Reizwertes, der als die Mitte zwischen zwei gegebenen Empfindungen unter der Herrschaft all dieser Einflüsse empfunden wird. Sie bietet somit keine Handbabe, um den der normalen Empfindungsmitte zugebörigen Reiz, wie es durch das psychophysische Interesse geboten wäre, zu ermitteln. Sie gewährt vielmehr bloß die Möglichkeit, nach theoretischer Feststellung der normalen Empfindungsmitte das Auftreten und die Wirksamkeit besonderer Einflüsse a uf die Beurteilung der mittleren Empfindung zu untersuchen.

\section{Die Beobachtungsreihen.}

Beachtet man das Versuchsverfahren, das bei den drei, von Fechner begründeten Methoden und bei der Methode der mittleren Abstufungen zur Verwendung kommen kann, so sieht man unmittelbar, daß in Wahrheit bloß zwei Methoden zur Gewinnung von Beobachtungsergebnissen zu unterscheiden sind. Entweder können die Reize, die zu irgendwie markierten Empfindungen gehören, hergestellt und gemessen werden; oder es kann nach oftmaliger Darbietung konstanter, einer schwankenden Beurteilung unterliegender Reize abgezählt werden, wieviel Urteile der einen oder anderen Art mit Rücksicht auf die einzelnen Reize gefällt worden sind. Dabei kann es sich im ersten Falle um die Herstellung anscheinend gleicher, eben merklich oder übermerklich verschiedener Reize bandeln. Es können ferner im letzteren Falle für einen einzelnen Hauptreiz oder für zwei Hauptreize konstante Vergleichsreize in geeigneter Weise gewählt werden, so daß die Vergleichsreize entweder bald dem einen Hauptreize gleich, 
bald von ihm verschieden erscheinen, oder bald in der Mitte zwischen den beiden Hauptreizen, bald dem einen oder dem anderen Hauptreize näher erscheinen.

Diese Einteilung hat zuerst Wundt in seiner "Logik" bei der Behandlung der Methoden der psychischen Größenmessung gegeben, wo er den Einstellungsmethoden die Abzählungsmethoden gegenüberstellte. Er weist dort allerdings außer der "Methode der Gleicheinstellung" (der Herstellung gleichbeurteilter Reize) und der "Methode der Einstellung gleicher Strecken" (der Herstellung des zur mittleren Empfindung gehörenden Reizes) auch die "Methode der Minimaländerungen" den Einstellungsmethoden zu, die nach den früheren Darlegungen ( $(9)$ vielmehr den Abzählungsmethoden zugehört, da sie bei einwandireier Handhabung, wie wir gesehen haben, eine Häufung und Auszählung der Urteile verlangt. In entsprechender Weise unterscheidet Ebbinghaus in seinen "Grundzügen der Psychologie" das Verfahren mit Reizfindung und das Verfahren mit Urteilsfindung. Desgleichen habe ich in meinem "Grundriß der Psychophysik" der kurzen Darlegung der psychophysischen Maßmethoden die Tremnung in Meßmethoden und Zählmethoden zugrunde gelegt. Eben dieselben Methoden nennt ferner G. E. Müller in seinen „Gesichtspunkten und Tatsachen der psychophysischen Methodik" die Methode der bestmöglichen Herstellung oder Herstellungsmethode und die Methode der konstanten Reize oder Konstanzmethode. Daneben hält er jedoch die Methode der eben merklichen Unterschiede in der umgestalteten Form als eine besondere „Grenzmethode" fest, ohne zwischen dem von ihm selbst angeregten Verfahren der Reizveränderung und Reizmessung zur Bestimmung eben merklicher und eben unmerklicher Unterschiede und dem von Wundt eingeführten Verfahren der Beurteilung vorbestimmter Reizabstufungen zu unterscheiden.

Als ein Verkennen der Bedeutung unserer $M a ß m e t h o d e n ~ m u ß$ es hingegen bezeichnet werden, wenn Abstufungsmethoden und Fehlermethoden unterschieden werden. Demn jede Methode hat das Obwalten mannigfach sich ändernder und relativ konstanter Einflüsse zu beachten, die in dem Spiel der Beobachtungsfehler und in der bei diesem Spiel sich geltend machenden Gesetzmäßigkeit hervortreten, so daß sie unter allen Umständen als eine 
Fehlermethode anzuerkennen ist, wofern sie überhaupt unter Berücksichtigung der Prinzipien der induktiven Wahrscheinlichkeitsbestimmung gehandhabt wird.

Die nach dem einen oder nach dem anderen Verfahren angestellten Beobachtungen können nun in ein bestimmtes Schema gebracht und so der weiteren Behandlung zugrunde gelegt werden.

Hat man durch Herstellung und Messung gleich beurteilter Reize Maßwerte in der hinreichend großen Anzahl $m$ erhalten, die sich über einen gewissen Bereich der Reizskala ausbreiten, so sind zunächst die Werte der Größe nach zu ordnen und in einer Tabelle zusammenzustellen. Da bei der Messung stets nur ein gewisser Grad von Genauigkeit erreichbar ist, so ist die ganze Reizskala in Intervalle geteilt zu denken, in denen der Genauigkeitsgrad seinen Ausdruck findet. Beispielsweise werden die Intervalle die Länge eines Millimeters oder eines Centimeters haben, je nachdem es sich um Längen handelt, die bis auf Millimeter oder bis auf Centimeter genau gemessen werden. Die Intervalle sind als gleich groß vorauszusetzen, weil bei der Messung aller Werte ein und derselbe Genauigkeitsgrad angestrebt werden muß. Will man den Genauigkeitsgrad nachträglich erniedrigen und die zunächst vorhandenen Intervalle vergrößern, so sind auch die vergrößerten Intervalle gleich lang zu machen. Erhält man auf diese Weise $\nu$ unmittelbar aneinander grenzende Intervalle, so sollen die Intervallmitten der Reihe nach durch

$$
a_{1}, a_{2}, a_{3} \ldots a_{\nu-1}, a_{v}
$$

bezeichnet werden. Die Intervalle selbst können, wenn ibre Länge gleich $2 l$ gesetzt wird, durch

$$
a_{1} \pm l, a_{2} \pm l, a_{3} \pm l \ldots a_{v} \pm l
$$

angedeutet werden. Auf diese Intervalle verteilen sich die gefundenen Maßwerte. Fällt einer gerade auf die Grenze zwischen zwei Nachbarintervalle, so ist er halb dem einen und halb dem anderen Intervalle zuzuweisen. Die Anzahlen der den einzelnen Intervallen zukommenden Maßwerte sollen durch

$$
z_{1}, z_{2}, z_{3} \ldots z_{v-1}, z_{v}
$$

angegeben werden, worunter Nullwerte und halbe Anzahlen anftreten können. 
Die Beobachtungen können somit tabellarisch in der Form

$$
\begin{array}{lllll}
a_{1} & a_{2} & a_{3} & \ldots & a_{v} \\
\hline z_{1} & z_{2} & z_{3} & \ldots & z_{v}
\end{array} \cdot \text {. . . . . . }
$$

wo $z_{*}$ die Anzahl der auf das Intervall $a_{*} \pm l$ fallenden Werte und

$$
m=z_{1}+z_{2}+z_{3}+\cdots+z_{v}
$$

die Summe aller Werte angibt, übersichtlich zusammengestellt werden.

Dieses Schema umfaßt alle nach der Methode der mittleren Fehler, der eben merklichen Unterschiede und der mittleren Abstufungen herstellbaren Beobachtungsreihen, bei denen es sich darum handelt, einen variierbaren Vergleichsreiz zu bestimmen, so daß er einer aufgestellten Norm entsprechend beurteilt wird.

\section{Fig. 4.}

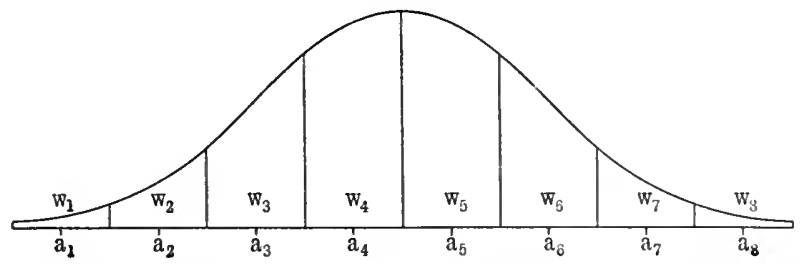

Der Gang der Werte $z_{1}, z_{2}, z_{3} \ldots z_{v}$ wird bei großem $v$ unregelmäßig sein, aber immerhin in der Regel eine von den beiderseitigen Enden nach der Mitte zunehmende Häufung erkennen lassen. Innerhalb der einzelnen Intervalle hat man zunächst die $z$ gleichmäßig verteilt zu denken. Ist aber ein gesetzmäßiger Gang der $z$-Werte ersichtlich und angebbar, so wird man auch die Verteilung der $z$ innerhalb der Intervalle dem Gesetze entsprechend vornehmen. Die geometrische Veranschaulichung läßt alsdann einen stetigen Kurvenzug hervortreten, für den nicht die absoluten Werte, sondern nur die relativen Werte

$$
w_{1}=\frac{z_{1}}{m} ; w_{2}=\frac{z_{2}}{m} ; \cdots v_{v}=\frac{z_{v}}{m}
$$

maßgebend sind. Beispielsweise findet in Fig. 4 eine aus acht Intervallen bestehende Tabelle, deren Werte dem gewöhnlichen Fehlergesetze folgen, ihre Veranschaulichung. 
Ist man hingegen auf die wiederholte Beurteilung gegebener Reize angewiesen, so müssen die Vergleichsreize, die zugleich mit der das Urteil bedingenden Norm dargeboten werden, den ganzen in Frage kommenden Bereich der Reizskala gleichmäßig ausfüllen und gleich oft in unregelmäßiger oder auch in regelmäßiger Folge beurteilt werden. Denn nur unter diesen Bedingungen kann eine vollständige und brauchbare Beobachtungsreihe resultieren. Die Vergleichsreize seien demgemäß gleich $a_{1}, a_{2}, a_{3} \ldots a_{v}$ und die Anzabl der auf jeden Vergleichsreiz fallenden Urteile sei gleich $m$. Als Norm kann ebenso wie beim vorigen Verfahren die Gleichheit oder die eben merkliche Verschiedenheit bezüglich einer gegebenen Empfindung oder die Mitte zwischen zwei gegebenen Empfindungen dienen. Man wird alsdann je nach Umständen den Vergleichsreiz $a_{x}(\varkappa=1,2,3 \ldots v)$ in Übereinstimmung mit der Norm oder im Sinne der größeren oder der kleineren Maßwerte von der Norm abweichend finden, wobei ein schwächeres oder stärkeres Abweichen stattfinden kann. Es lassen sich demgemäß die Urteile durch ,gleich" $(=)$, ,größer" $(>)$, „kleiner" $(<)$ und nötigenfalls durch „eben merklich größer" $(>)$ und "deutlich größer" $(\Varangle)$, „eben merklich kleiner" $(<)$ und "deutlich kleiner" $(\Varangle)$ unterscheiden. Die Anzahl der Urteile "gleich", "größer" und "kleiner", die sich für den Vergleichsreiz $a_{x}$ ergeben, sollen durch $z_{x}^{\prime}, p_{\%}, n_{x}$ und bei Unterscheidung eben merklicher und deutlicher Verschiedenheit durch $p_{\times 1}, p_{x_{2}}$ und $n_{x_{1}}, n_{x_{2}}$ bezeichnet werden.

Die Beobachtungen können demgemäß nach dem Schema

oder

\begin{tabular}{|c|c|c|c|c|}
\hline & $<$ & $=$ & $>$ & \\
\hline$a_{1}$ & $n_{1}$ & $z_{1}^{\prime}$ & $p_{1}$ & \\
\hline$a_{2}$ & $n_{2}$ & $z_{2}^{\prime}$ & $p_{2}$ & \\
\hline$a_{3}$ & $n_{3}$ & $z_{3}^{\prime}$ & $p_{3}$ & . . \\
\hline . & . & $\cdot$ & . & \\
\hline . & . & $\cdot$ & . & \\
\hline . & . & . & . & \\
\hline$a_{v}$ & $n_{v}$ & $z_{v}^{\prime}$ & $p_{v}$ & \\
\hline
\end{tabular}




\begin{tabular}{|c|c|c|c|c|c|c|}
\hline & \multirow[b]{2}{*}{$\Varangle$} & \multicolumn{2}{|c|}{85} & \multirow[b]{2}{*}{$>$} & \multirow[b]{2}{*}{$x$} & \\
\hline & & $<$ & $=$ & & & \\
\hline$a_{1}$ & $n_{12}$ & $n_{11}$ & $z_{1}^{\prime}$ & $p_{11}$ & $p_{12}$ & \\
\hline$a_{2}$ & $n_{22}$ & $n_{21}$ & $z_{2}^{\prime}$ & $p_{21}$ & $p_{22}$ & \\
\hline$a_{3}$ & $n_{32}$ & $n_{31}$ & $z_{3}^{\prime}$ & $p_{31}$ & $p_{32}$ & . $\cdot\left(\mathrm{B}_{1}\right)$ \\
\hline . & • & $\cdot$ & $\cdot$ & $\cdot$ & $\cdot$ & \\
\hline . & . & . & . & . & . & \\
\hline . & . & • & . & . & . & \\
\hline$a_{y}$ & $n_{v_{2}}$ & $n_{1}$ & $z_{v}^{\prime}$ & $p_{v 1}$ & $p_{v_{2}}$ & \\
\hline
\end{tabular}

geordnet und übersichtlich zusammengestellt werden, je nachdem die Scheidung in eben merkliche und deutliche Verschiedenheit unterbleibt oder vollzogen wird.

Dieses Schema umfaßt sowohl die nach der Methode der Minimaländerungen wie auch die nach der Methode der richtigen und falschen Fälle bei sachgemäßer Beobachtungsweise sich ergebenden Beobachtungsreihen.

Man kann nun den Gang der $z$-Werte in (A) und in (B) odor $\left(B_{1}\right)$ betrachten und durch graphische Darstellung (wie in Fig. 4) veranschaulichen. Und es ist wohl möglich, gewisse Aufschlüsse aus dem unmittelbaren Anblick der Streuung und des mehr oder minder regelmäßigen Ganges der Werte zu erhalten. Insbesondere lassen sich in (B) bei hinreichend regelmäßigem Gange der $n$ - und $p$-Werte, nötigenfalls durch Interpolation, die Stellen ermitteln, wo nach den früheren Erörterungen (S. 58) durch die Häufigkeitswerte $n=1 / 2 m$ und $p=1 / 2 m$ der untere und obere Unterschiedsschwellenwert angezeigt wird, ohne daß jedoch diese Bestimmung, solange die Angabe der ihr zukommenden Sicherheit fehlt, unbedingtes Zutrauen verdient.

Will man aber die Beobachtungsergebnisse einer rationellen Behandlung unterwerfen, so ist vor allem der (S. 76) angegebene fehlertheoretische Zusammenhang zwischen der Methode der mittleren Fehler einerseits und der Methode der richtigen und falschen Fälle andererseits zu beachten. Er fordert eine einheitliche Behandlungsweise der Beobachtungsergebnisse, mögen sie durch das Messen gleich beurteilter Reize oder durch das wiederholte Beurteilen gegebener Reize gewonnen worden sein.

Bezeichnet man nämlich die zu den Vergleichsreizen $a_{1}, a_{2}$, $a_{3}, \ldots a_{v}$ gehörige, für den Bereich dieser Werte näherungsweise 
als konstant vorauszusetzende Unterschiedsschwelle durch $i$, wonach dem Reize $a_{x}$ das Reizgebiet $a_{x} \pm i$ zugehört, so muß bei der Darbietung des Reizes $a_{x}$ das Urteil "kleiner", "gleich" oder "größer" erfolgen, je nachdem unter den nämlichen obwaltenden Einflüssen beim Herstellungsverfahren ein Reizwert oberhalb $a_{x}+i$, zwischen $a_{x}+i$ und $a_{x}-i$ oder unterhalb $a_{x}-i$ sich ergeben würde. Gliedert man überdies dem Intervalle $a_{x} \pm i$ nach oben und nach unten das Intervall an, das die im Vergleich mit $a_{x}$ eben merklich größeren und eben merklich kleineren Reizwerte umschließt, so muß ferner das Urteil eben merklich kleiner oder deutlich kleiner, eben merklich größer oder deutlich größer abgegeben werden, je nachdem der in Übereinstimmung mit der Norm hergestellte Reiz nach der entgegengesetzten Richtung in das Nachbarintervall oder in das weiter entfernte Gebiet fallen würde.

Wird $i=l$ angenommen, so ist demgemäß

$$
\left.\begin{array}{l}
n_{x}=z_{x+1}+z_{x+2}+\cdots+z_{v} \\
z_{x}^{\prime}=z_{x} \\
\mu_{x}=z_{x-1}+z_{x-2}+\cdots+z_{1}
\end{array}\right\} . .
$$

Ist hingegen $i>l$, so wird

$$
\left.\begin{array}{l}
n_{x}<z_{x+1}+z_{x+2}+\cdots+z_{y} \\
z_{x}^{\prime}>z_{x} \\
p_{x}<z_{x-1}+z_{x-2}+\cdots+z_{1}
\end{array}\right\} . .
$$

Ist schließlich $i<1$, so wird

$$
\left.\begin{array}{l}
u_{x}>z_{x+1}+z_{x+2}+\cdots+z_{y} \\
z_{x}^{\prime}<z_{x} \\
p_{x}>z_{x-1}+z_{x-2}+\cdots+z_{1}
\end{array}\right\} . . .
$$

Man überblickt diese Beziehungen unmittelbar in Fig. 4, wenn man den acht Werten $a_{1}, a_{2} \ldots a_{3}$ Unterschiedsschwellen beilegt, die einmal gleich den dort eingezeichneten Intervallen sind, sodann über dieselben hinausragen und schließlich innerhalb derselben bleiben. Man erkennt auch ohne weiteres, daß die Proportion

$$
z_{x}^{\prime}: z_{x}=i: 7 \text {. . . . . . }
$$


mit hinreichender Annäherung gilt, so daß, wenn die über $x=1$, 2, $3 \ldots m$ erstreckte Summe durch $\Sigma$ angedeutet wird,

$$
\Sigma z_{r}^{\prime}: \Sigma z_{x}=i: l \text {. . . . . . }
$$

oder, da $\Sigma z_{\%}=m$,

$$
i=\frac{l}{m} \Sigma z_{*}^{\prime} \cdot \cdot \cdot \cdot \cdot \cdot \cdot \cdot \cdot
$$

gesetzt werden darf. Es wird so eine Bestimmung der Unterschiedsschwelle, allerdings ohne Angabe der ihr zukommenden Sicherheit, gewonnen.

Fingiert man den Fall, daß die Beobachtungen keinen wechselnden Einflüssen, sondern lediglich der Herrschaft der Unterschiedsschwelle unterworfen sind, so verteilen sich die $z$-Werte in (A) bei der Annahme, daß $i=l$ sei, gleichmäßig auf ein durch die Entfernung der oberen und unteren Unterschiedsschwelle bestimmtes Gebiet von der Länge $2 i=27$; und im Zusammenhange damit wird ein entsprechendes Gebiet in (B) mit Urteilszahlen $n=0 ; z=m ; p=0$ erfüllt, dem sich einerseits die Urteilszahlen $n=m ; z=p=0$, andererseits die Urteilszahlen $n=z=0 ; p=m$ anschließen. Ist $i$ von $l$ verschieden, so wird der Übergang aus dem mittleren Gebiete in die angrenzenden Gebiete durch ein Übergangsintervall vermittelt. Durch die

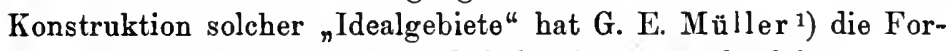
mel (47), ebenfalls ohne Sicherheitsbestimmung, abgeleitet.

Die Relationen (42), (43), (44) lassen erkennen, daß in der Tat die in (A) einerseits und in (B) oder $\left(B_{1}\right)$ andererseits vorliegenden Beobachtungsreihen in ihrer Gesetzmäßigkeit übereinstimmen müßten, wenn es möglich wäre, nach dem einen und dem anderen Verfahren unter völlig vergleichbaren Umständen zu beobachten. Sie lehren aber außerdem, daß die einzelnen Reihen von (B) und von $\left(B_{1}\right)$ nicht unabhängig voneinander sind. Denn ein und dasselbe Gesetz beherrscht normalerweise den Gang der $z_{x}$, der $p_{x}, z_{x}^{\prime}, n_{x}$ und der $p_{1 x}, p_{2 x}, n_{1 x}, n_{2 x}$, wofern überhaupt von einẹm gesetzmäßigen Gange die Rede sein kann. Es müßte beispielsweise, wenn das Gausssche Fehlergesetz vorausgesetzt würde, ein und dasselbe Präzisionsmaß $h$ für jede S. 148 .

1) Tatsachen und Gesichtspunkte der psychophysischen Methodik, 
Reihe zugrunde gelegt werden. Wäre dies nicht durchführbar, so dürfte auch das angenommene Fehlergesetz nicht verwendet werden ${ }^{1}$ ).

Es kommt aber hier nicht in Betracht, ob die Reihen in (A) und in $(B)$ oder in $\left(B_{1}\right)$ hinsichtlich ihrer empirisch bestimmten Werte untereinander mehr oder weniger übereinstimmen. Das Wesentliche ist vielmehr, daß vom Standpunkte der Theorie aus die Übereinstimmung aller Reihen und somit ihre Reduzierung auf eine gemeinsame, einer und derselben Behandlung zugängliche Form gefordert werden muß.

Dieser Forderung wird man gerecht, wenn man in Übereinstimmung mit (45) aus den Werten $z_{x}^{\prime}$ die Werte

$$
z_{x}=\frac{l}{i} z_{x}^{\prime}
$$

berechnet und so aus den $z^{\prime}$-Werten in (B) die in das Schema (A) sich einfügende Reihe

$$
\left.\begin{array}{ccccc}
a_{1} & a_{2} & a_{3} & \ldots & a_{v} \\
\frac{l}{i} z_{1}^{\prime} & \frac{l}{i} z_{2}^{\prime} & \frac{l}{i} z_{3}^{\prime} & \ldots & \frac{l}{i} z_{v}^{\prime}
\end{array}\right)
$$

ableitet. Man gewinnt beispielsweise aus Tabelle 3 (S. 57) die Reihe

\begin{tabular}{ccccccccc}
2 & 3 & 4 & 5 & 6 & 7 & 8 & 9 & 10 \\
\hline 1 & 2 & 3 & 3 & 3 & 4 & 2 & 2 & 0,5
\end{tabular},

indem die Werte $z^{\prime}$ mit dem Koeffizienten $l: i=19: 81$ (angenähert $=1: 4$ ) multipliziert werden. Entsprechende Reihen kann man aus den Werten $n_{x_{1}}$ und $p_{x_{1}}$ in $\left(\mathrm{B}_{1}\right)$ bilden, wenn man die Koeffizienten $\alpha$ und $\beta$ so bestimmt, daß

$\alpha n_{11}+\alpha n_{21}+\cdots+\alpha n_{v 1}=m ; \beta p_{11}+\boldsymbol{\beta} p_{21}+\cdots+\beta p_{11}=m$ und die Werte $\alpha n_{x 1}$ und $\beta p_{x 1}$ in die Reihen

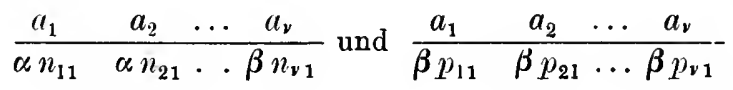

1) Vgl. hierzu G. E. Müller, Psychoph. Methodik, S. 71, wo für die obere und untere Unterschiedsschwelle verschiedene Präzisionsmaße angegeben werden. 
ordnet. Sie sind indessen im allgemeinen mit größeren Unregelmäßigkeiten als die Reihen $\left(A_{1}\right)$ behaftet, da sich die Werte, die eben merklich kleiner und eben merklich größer erscheinen, nur unbestimmt von den deutlich kleineren und deutlich größeren Werten absondern lassen. - Man kann ferner auch aus den Differenzen der aufeinanderfolgenden $n_{x}$ und $p_{x}$ oder $n_{x 2}$ und $p_{* 2}$ analoge Reihen herstellen, die indessen nicht als neue Beobachtungsreihen aufzufassen sind.

\section{Das Fehlergesetz.}

Hat man nun die beobachteten Werte in eine dem Schema (A) entsprechende Reihe gebracht und sollen weitere Bestimmungen ausgeführt werden, so erhebt sich die Frage nach dem Verteilungsgesetz, das als Ersatz für das unzureichende Gausssche Fehlergesetz dienen kann.

Der Vorschlag von G. E. Müller ${ }^{1}$ ), die Wahrscheinlichkeitsgesetze

$$
y=\frac{2}{\alpha} \sin ^{2}\left[\frac{\pi}{2}\left(1-\frac{\delta}{\alpha}\right)\right] \text { oder } y=\frac{3}{2 \alpha}\left(1-\frac{\delta^{2}}{\alpha^{2}}\right)
$$

oder andere der Art zu wählen, ist unannehmbar. Er würde die Fehlertheorie auf den (S. 36 u. 37 gekennzeichneten) Standpunkt vor dem Auftreten von Gauss und Laplace zurückführen, da ein bestimmtes endliches Fehlermaximum vorausgesetzt würde und als Präzisionsmaß benutzt werden müßte.

Es sagt allerdings Gauss selbst nach Ableitung seines Fehlergesetzes in der "Theoria motus corporum coelestium" (Artikel 178): ,Die soeben ermittelte Funktion kann zwar in aller Strenge die Wahrscheinlichkeiten der Fehler sicher nicht darstellen; denn da die möglichen Fehler immer in bestimmten Grenzen eingeschlossen sind, so müßte die Wahrscheinlichkeit größerer Fehler sich immer $=0$ ergeben, während unsere Formel immer einen endlichen Wert liefert." Er weist aber auch darauf hin, daß "die Natur der Sache" niemals eine völlig strenge Bestimmung der Fehlergrenzen gestatte.

Es muß indessen ein asymptotischer Verlauf der Fehlerkurve, wie ihn das Gausssche Feblergesetz zeigt, den Grundsätzen der

1) Pflügers Archiv 19, 209 und Psychophysische Methodik, S. 90. 
induktiven Wahrscheinlichkeitsbestimmung zufolge geradezu gefordert werden. Denn wio das Bernoullische Theorem lehrt, können niemals Einzelwerte, sondern nur Mittelwerte als Repräsentanten ganzer Mannigfaltigkeiten von Werten, die sich bei unbegrenzter Wiederholung der Beobachtungen ergeben würden, bestimmt werden. Darum kann auch das Feblermaximum bloß als Vertreter einer Mannigfaltigkeit von Fall zu Fall sich ergebender maximaler Werte auftreten, so daß ein absolut größter Wert - das wahre Maximum - in der Tat unangebbar und somit, mit unendlich geringer Wahrscheinlichkeit behaftet, in unbestimmter oder unbegrenzter Größe vorauszusetzen ist. Nur dann, wenn die Erstreckung des Fehlerbereichs von vornherein bekannt wäre und nicht erst auf Grund der Beobachtungen festgestellt werden müßte, könnte die Fehlergrenze als scharf bestimmter Wert auftreten.

Demgemä $\beta$ ist bei der Aufstellung von Fehlergesetzen der Grundsatz zu befolgen, daß nur Mittelwerte, aber niemals Einzelwerte, also a uch insbesondere nicht die extremen Werte aus den Beobachtungen abzuleiten und als Parameter, die den Verlauf der Fehlerkurve bestimmen, zu benutzen sind.

Darum sind auch die Gesetze, die Pearson in seinen als "Mathematische Theorie der Entwickelung" bezeichneten Untersuchungen aufstellt, nicht verwendbar. Dieser Forscher stellt sich nämlich gerade die Aufgabe, deren Lösung wir soeben als unmöglich bezeichnet haben: die Begrenztheit oder Unbegrenztheit einer Reihe von Gliedern, deren Auftreten durch Wahrscheinlichkeitsbestimmungen geregelt ist, zu ermitteln. Er sagt ${ }^{1}$ ): „Wir können die Ausdehnung einer Reihe nicht a priori angeben, aber wir sind gewiß, daß sie existiert, und sie ist eine Größe, die aus den Beobachtungen selbst, ebenso wie das arithmetische Mittel und die mittlere Abweichung, bestimmt werden kann." Der Erfolg dieser prinzipiell unzulässigen Annahme ist die Aufstellung von fünf Kurventypen:

1) Contributions to the mathematical theory of evolution; Philos. Transactions Roy. Soc. London, 186 A, 1895, S. 359. - Eine eingehendere Kritik der Aufstellungen Pearsons gebe ich in meiner "Theorie der Kollektivgegenstände" (1902), S. 79. 
1. beiderseitig begrenzte asymmetrische Kurven von der Form :

$$
y=y_{0}\left(1+x / a_{1}\right)^{v a_{1}}\left(1-x / a_{2}\right)^{v a_{2}}
$$

2. beiderseitig begrenzte symmetrische Kurven von der Form:

$$
y=y_{0}\left(1-x^{2} / a^{2}\right)^{m}
$$

3. einseitig begrenzte Kurven:

$$
y=y_{0}(1+x / a) \cdot e x p(-\gamma x)
$$

4. beiderseits unbegrenzte, aber unsymmetrische Kurven:

$$
y=y_{0} \cos ^{2 n} \boldsymbol{\vartheta} . \exp (\boldsymbol{- \nu} \boldsymbol{\vartheta}) ; x=\operatorname{atang} \vartheta
$$

5 . beiderseits begrenzte, symmetrische Kurven, die das gewöhnliche Fehlergesetz befolgen. Dem aufgestellten Grundsatze zufolge könnte nur die vierte Form in Betracht kommen. Sie dürfte aber zu keiner bequemen Behandlung der Beobachtungsreihen führen und würde sich wohl nur in beschränktem Maße als zutreffend erweisen.

Frei von Verstößen gegen die Grundsätze der induktiven Wahrscheinlichkeitsbestimmung sind hingegen die Veraligemeinerungen des Gaussschen Fehlergesetzes, die Fechner in seinem nachgelassenen Werke "Kollektivmaßlehre" (1897) gibt. Sie beruhen auf der Einsicht, daß bei den empirisch bestimmten Reihen von Wahrscheinlichkeitswerten, welche die sog. Kollektivgegenstände $\left.{ }^{1}\right)$ darbieten, an Stelle der von dem Fehlergesetze geforderten Symmetrie vielmehr Asymmetrie als Regel zu gelten habe. Dann ist das arithmetische Mittel nicht mehr der wahrscheinlichste Wert, und die Abweichungen sind demzufolge statt rom arith-

1) Unter einem Kollektivgegenstande versteht Fechner (Kollektivmaßlehre, S. 3) „einen Gegenstand, der aus unbestimmt vielen, nach Zufall variierenden Exemplaren besteht, die durch einen Art- oder Gattungsbegriff zusammengehalten werden. So bildet der Mensch einen Kollektivgegenstand im weiteren Sinne, der Mensch von bestimmtem Geschlecht, bestimmtem Alter und bestimmter Rasse einen solchen im engeren Sinne". Anthropologie, Zoologie, Botanik, auch die Meteorologie in ihren nicht periodischen Witterungsphänomenen haben es „überhaupt wesentlich mit Kollektivgegenständen zu tun, da es sich darin nicht um eine Charakteristik einzelner Exemplare, sondern nur um das handeln kann, was einer Gesamtheit derselben zukommt, die aus diesen oder jenen Gesichtspunkten als Gattung oder Art in größerer oder geringerer Weite zusammengefaßt wird . 
metischen Mittel vielmehr von dem wahrscheinlichsten Werte, den Fechner den "dichtesten Wert" nennt, zu rechnen. Dabei wird angenommen, daß die Verteilung der Abweichungen beiderseits vom dichtesten Werte, für die eine Seite unabhängig von der anderen, dieselbe Regel befolgt, die bei symmetrischer Verteilung für beide Seiten gemeinsam von den Abweichungen bezüglich des arithmetischen Mittels auf Grund des Gaussschen Gesetzes befolgt wird.

Es steht nun nichts im Wege, dieses zweiseitige oder, wie Fechner sagt, „zweispaltige Gausssche Gesetz ${ }^{\text {s }}$ an Stelle des einfachen Gesetzes zu verwenden, wo eine asymmetrische Gruppierung der Werte in (A) oder (B) einen Erfolg verspricht. Man wird aber an ihm so wenig wie an dem beiderseits unbegrenzten asymmetrischen Kurventypus von Pearson ein in jedem Falle ausreichendes Hilfsmittel zur Darstellung der Beobachtungsreihen finden.

Es handelt sich nämlich überhaupt nicht darum, spezielle Gesetze aufzustellen, die in dem einen Falle zutreffen und in einem anderen Falle versagen. Es gilt vielmehr, eine Darstellungsweise zu finden, die für jede Beobachtungsreihe mit einem beliebigen, je nach Bedürfnis bald größeren, bald kleineren Grade von Genauigkeit anwendbar ist. Wird nämlich ein bestimmtes Verteilungsgesetz angenommen und zur Darstellung einer vorgegebenen Beobachtungsreihe benutzt, so kann nur die Frage zur Entscheidung gebracht werden, ob der in dem angenommenen Gesetze hervortretende Typus in größerem oder geringerem Maße zur Verwirklichung gelangt. Wichtiger ist es aber, den in der Beobachtungsreihe tatsächlich vorhandenen Typus auf Grund der empirisch gefundenen Wahrscheinlichkeits- oder Häufigkeitswerte unmittelbar zu bestimmen.

Um dies zu ermöglichen, hat Bruns eine allgemein gültige Methode zur Darstellung von Fehlergesetzen entwickelt. Er erläutert') sein Verfahren durch den Hinweis auf die Darstellung von Tönen mittels Fourierscher Reihen von der Form

$$
\begin{aligned}
y=a & +a_{1} \cos x+a_{2} \cos 2 x+a_{3} \cos 3 x+\cdots \\
& +b_{1} \sin x+b_{2} \sin 2 x+b_{3} \sin 3 x+\cdots
\end{aligned}
$$

1) „Kur Kollektivmaßlehre"; Philosophische Studien 14, 347. 
wo die Koeffizienten $a$ und $b$ die Stärke und Klangfarbe des Tones bestimmen, während die Höhe desselben von $x$ abhängt. Die Anzahl der auftretenden Reihenglieder ist streng genommen und von besonderen Fällen abgesehen unendlich groß, jedoch kommt bei der Darstellung eines beobachteten Tones nur eine beschränkte Anzahl von Koeffizienten als merklich in Betracht, so daß die übrigen ohne Nachteil gleich Null gesetzt werden dürfen". "Es ist somit die Bestimmung einer endlichen, aber im voraus nicht feststehenden Anzahl vou Gliedern ausreichend. In ähnlicher Weise kann man aber auch, wie Bruns weiterhin bemerkt, bezüglich der Beobachtungsreihen verfahren. Bezeichnet man die beobachteten Werte mit $a$, einen bestimmten mittleren Wert mit $c$, die von diesem Werte gerechneten Abweichungen oder Fehler mit $\Delta$, mit $h$ eine dem Parameter des gewöhnlichen Fehlergesetzes entsprechende Konstante, mit $\Phi(h \Delta)$ eine gewisse Funktion, die unter Umständen das gewöhnliche Fehlergesetz darstellen kann, mit $\Phi_{1}(h \Delta), \Phi_{2}(h \Delta), \Phi_{3}(h \Delta) \ldots$ die aufeinanderfolgenden Ableitungen von $\Phi$, schließlich mit $A, A_{1}, A_{2}$, $A_{3} \ldots$ ein gewisses System von Koeffizienten, so lält sich die Häufigkeit $y$ für das Auftreten des Fehlers $\Delta$ in der Form

$$
y=A \Phi(h \Delta)+A_{1} \Phi_{1}(h \Delta)+A_{2} \Phi_{2}(h \Delta)+\cdots
$$

darstellen. Es handelt sich nun nicht mehr um den Nachweis, da $B$ die Beobachtungsreibe "auf einen bestimmten einfachen Kurventypus", etwa das zweiteilige Gaussche Gesetz, zurückgeführt werden kann, sondern darum, jedesmal „das für die Beobachtungsreihe charakteristische Wertensystem der Größen $h, c, A$ zu ermitteln" und die weitere Untersuchung, auf die Beschaffenheit dieses Wertensystems" zu gründen.

\section{Die Mittelwerte der Beobachtungsreihen.}

Einer Anwéndung dieses Verfahrens steht nur die Umständlichkeit der hierzu notwendigen Berechnungen im Wege. Es verdient darum beachtet zu we:den, daß eine Darstellung der Beobachtungsreihen möglich ist, ohne die Form (48) oder eine ähnliche Form zugrunde zu legen. 
$\mathrm{Zu}$ diesem $\mathrm{Zwecke}$ sollen die äquidistanten, den Intervallen von der Länge $2 l$ zugehörigen Werte $a_{1}, a_{2}, a_{3} \ldots a_{v}$ nach Schema (A) in den absoluten Häufigkeiten $z_{1}, z_{2}, z_{3} \ldots z_{v}$ oder in den relativen Häufigkeiten $w_{1}=z_{1}: m ; w_{2}=z_{2}: m$; $\ldots w_{v}=z_{v}: m$ vorausgesetzt werden, wo $m$ die Summe aller $z$ angibt. Ich bezeichne nun einen beliebig, aber fest gewählten Wert der Reihe $a_{1}, a_{2} \ldots a_{v}$ durch $a_{x}$ und bilde die Summen

$$
\begin{aligned}
m & =z_{1}+z_{2} \\
m \eta(1) & =z_{1}\left(a_{1}-a_{x}\right)+z_{2}\left(a_{2}-a_{x}\right)+\cdots+z_{v} \\
m \eta(2) & =z_{1}\left(a_{1}-a_{x}\right)^{2}+z_{2}\left(a_{2}-a_{x}\right)^{2}+\cdots+z_{v}\left(a_{v}-a_{x}\right) \\
m &
\end{aligned}
$$

so $\mathrm{da} ß$ allgemein für $\varrho=0,1,2,3 \ldots$

$$
\begin{aligned}
& m \eta(\varrho)=z_{1}\left(a_{1}-a_{*}\right)^{0}+z_{2}\left(a_{2}-a_{x}\right)^{0}+\cdots+z_{v}\left(a_{\nu}-a_{*}\right)^{9} \\
& \text { oder } \\
& \left.\eta(\varrho)=w_{1}\left(a_{1}-a_{x}\right)^{\rho}+w_{2}\left(a_{2}-a_{x}\right)^{\rho}+\cdots+v_{\nu}\left(a_{v}-a_{x}\right)^{2}\right)
\end{aligned}
$$

Setzt man ferner

so erhält man

$$
\eta_{o}^{?}=\eta(0)
$$

$$
\eta_{\varphi}=\stackrel{\rho}{\sqrt{r}}\left\{w_{1}\left(a_{1}-a_{x}\right)^{\varphi}+w_{2}\left(a_{2}-a_{x}\right)^{\rho}+\cdots+w_{\nu}\left(a_{\nu}-a_{x}\right)^{\rho}\right\}
$$

Ich nenne $\eta(\varrho)$ die auf den Ausgangswert $a_{x}$ bezogene Mittelwertpotenz und $\eta_{0}$ den auf den Ausgangswert $a_{x}$ bezogenen Mittelwert $\varrho$-ter Ordnung der gegebenen Beobachtungsreihe.

Da die Differenzen $a_{1}-a_{*}, a_{2}-a_{*} \ldots a_{\nu}-a_{*}$ Vielfache von $2 l$ sind, indem

$a_{1}-a_{x}=(1-x) 2 l ; a_{2}-a_{x}=(2-x) 2 l ; \ldots a_{\nu}-a_{x}=(\nu-x) 2 l$, so gewinnt man insbesondere die Gleichungen

$$
\begin{aligned}
m & =\cdots+\quad z_{x-2}+\quad z_{x-1}+z_{x}+z_{x+1}+\cdots \\
m \eta(2) & =\cdots+2^{2} \cdot(2 l)^{2} \cdot z_{x-2}+(2 l)^{2} \cdot z_{x-1}+0+(2 l)^{2} \cdot z_{x+1}+\cdots \\
m \eta(4) & =\cdots+2^{4} \cdot(2 l)^{4} \cdot z_{x-2}+(2 l)^{4} \cdot z_{x-1}+0+(2 l)^{4} \cdot z_{x+1}+\cdots \\
m \eta(6) & =\cdots+2^{6} \cdot(2 l)^{6} \cdot z_{x-2}+(2 l)^{6} \cdot z_{x-1}+0+(2 l)^{6} \cdot z_{x+1}+\cdots
\end{aligned}
$$

Man erhält hieraus durch beiderseitige Multiplikation mit den Faktoren:

$$
1 ;-\frac{\pi^{2}}{3 !(27)^{2}} ;+\frac{\pi^{4}}{5 !(27)^{4}} ;-\frac{\pi^{6}}{7 !(27)^{6}} ;+\frac{\pi^{s}}{9 !(2 l)^{8}} \text { usw. }
$$


das System:

$m=\cdots+z_{x-2}+z_{x-1}+z_{x}+z_{x+1}+\cdots$

$-m \eta(2) \frac{\pi^{2}}{3 !(2 l)^{2}}=\cdots-\frac{2^{2} \pi^{2}}{3 !} z_{x-2}-\frac{\pi^{2}}{3 !} z_{x-1}-0-\frac{\pi^{2}}{3 !} z_{x+1}-\cdots$ $m \eta(4) \frac{\pi^{4}}{5 !(2 l)^{4}}=\cdots+\frac{2^{4} \pi^{4}}{5 !} z_{x-2}+\frac{\pi^{4}}{5 !} z_{x-1}+0+\frac{\pi^{4}}{5 !} z_{x+1}+\cdots$

$-m \eta(6) \frac{\pi^{6}}{7 !(2 l)^{6}}=\cdots-\frac{2^{6} \pi^{6}}{7 !} z_{x-2}-\frac{\pi^{6}}{7 !} z_{x-1}-0-\frac{\pi^{6}}{7 !} z_{x+1}-\cdots$

Es ist aber

$$
1-\frac{\lambda^{2} \pi^{2}}{3 !}+\frac{\lambda^{4} \pi^{4}}{5 !}-\frac{\lambda^{6} \pi^{6}}{7 !}+\cdots=\frac{\sin (\lambda \pi)}{\lambda \pi}
$$

so daß für $\lambda=0$ der Wert 1 und für jedes von Null verschiedene, positive oder negative ganzzahlige $\lambda$ der Wert 0 sich ergibt. Durch Summation der untereinanderstehenden Glieder verschwinden daher auf der rechten Seite alle $z$-Werte bis auf $z_{x}$. Man gelangt somit zu der Darstellungsform:

$$
\left.\begin{array}{r}
z_{\varkappa}=m-m \eta(2) \frac{\pi^{2}}{3 !(2 l)^{2}}+m \eta(4) \frac{\pi^{4}}{5 !(2 l)^{4}} \\
-m \eta(6) \frac{\pi^{6}}{7 !(2 l)^{6}}+\cdots \\
w_{x}=1-\eta(2) \frac{\pi^{2}}{3 !(2 l)^{2}}+\eta(4) \frac{\pi^{4}}{5 !(2 l)^{4}} \\
-\eta(6) \frac{\pi^{6}}{7 !(2 l)^{6}}+\cdots
\end{array}\right\}
$$

Hier sind die Mittelwertpotenzen $\eta(2), \eta(4), \eta(6) \ldots$ für den jeweils gewählten Ausgangswert $a_{\%}$ zu berechnen, so daß auf diese Weise die funktionelle Abbängigkeit der $z_{x}$ und $v_{x}$ von den $a_{x}$ zutage tritt. Diese Abhängigkeit tritt in der Darstellung unmittelbar hervor, wenn man einen ganz beliebig gewählten reellen Zahlenwert durch $a_{0}$ bezeichnet, die Differenzen

durch

$$
a_{1}-a_{x} ; \quad a_{2}-a_{x} ; \quad \ldots \quad a_{v}-a_{x}
$$

$\left(a_{1}-a_{0}\right)-\left(a_{x}-a_{0}\right) ;\left(a_{2}-a_{0}\right)-\left(a_{x}-a_{0}\right) ; \ldots\left(a_{2}-a_{0}\right)-\left(a_{x}-a_{0}\right)$ 
ersetzt und die $\eta$-Werte nach Potenzen von $a_{x}-a_{0}$ entwickelt. Setzt man nämlich für $\varrho=0,1,2,3 \ldots$

$$
m \varepsilon(\varrho)=z_{1}\left(a_{1}-a_{0}\right)^{\rho}+z_{2}\left(a_{2}-a_{0}\right)^{\rho}+\cdots+z_{v}\left(a_{v}-a_{0}\right)^{\circ}
$$

so daß für den Ausgangswert $a_{0}$ durch $\varepsilon(\varrho)$ die Mittelwertpotenz $\varrho$-ter Ordnung und durch

$$
\varepsilon_{\varphi}=\sqrt[0]{\varepsilon(\varrho)} \text {. }
$$

der Mittelwert $\varrho$-ter Ordnung angegeben wird, so erhält man mit Rücksicht auf

$$
\begin{aligned}
\left(a_{\lambda}-a_{x}\right)^{0} & =\left(a_{\lambda}-a_{0}\right)^{o}-\frac{\varrho}{1}\left(a_{\%}-a_{0}\right)\left(a_{\lambda}-a_{0}\right)^{0-1} \\
& +\frac{\rho(\varrho-1)}{1.2}\left(a_{\varkappa}-a_{0}\right)^{2}\left(a_{\lambda}-a_{0}\right)^{\rho-2}-\cdots
\end{aligned}
$$

zunächst

$$
\begin{aligned}
\eta(\varrho) & =\varepsilon(\varrho)-\frac{\varrho}{1}\left(a_{x}-a_{0}\right) \varepsilon(\varrho-1) \\
& +\frac{\varrho(\varrho-1)}{1.2}\left(a_{x}-a_{0}\right)^{2} \varepsilon(\varrho-2)-\cdots \pm\left(a_{x}-a_{0}\right)^{\rho}
\end{aligned}
$$

und somit

$$
\left.\begin{array}{l}
w_{x}=1-\frac{\pi^{2}\left(a_{x}-a_{0}\right)^{2}}{3 !(2 l)^{2}}+\frac{\pi^{4}\left(a_{x}-a_{0}\right)^{4}}{5 !(2 l)^{4}}-\cdots \\
-\varepsilon(1)\left\{-\frac{2 \pi^{2}\left(a_{x}-a_{0}\right)}{3 !(2 l)^{2}}+\frac{4 \pi^{4}\left(a_{x}-a_{0}\right)^{3}}{5 !(2 l)^{4}}-\cdots\right\} \\
+\frac{\varepsilon(2)}{1.2}\left\{-\frac{1.2 . \pi^{2}}{3 !(2 l)^{2}}+\frac{4.3 . \pi^{4}\left(a_{x}-a_{0}\right)^{2}}{5 !(2 l)^{4}}-\cdots\right\}
\end{array}\right\}
$$

Sohreibt man

$$
\begin{aligned}
\varphi\left(a_{*}\right) & =1-\frac{\pi^{2}\left(a_{x}-a_{0}\right)^{2}}{3 !(2 l)^{2}}+\frac{\pi^{4}\left(a_{x}-a_{0}\right)^{4}}{5 !(2 l)^{4}}-\cdots \\
& =\frac{2 l}{\pi\left(a_{x}-a_{0}\right)} \cdot \sin \frac{\pi\left(a_{x}-a_{0}\right)}{2 l}
\end{aligned}
$$

und bezeichnet man die aufeinanderfolgenden Ableitungen von $\varphi\left(a_{*}\right)$ nach $a_{*}$ durch $\varphi_{1}\left(a_{*}\right), \varphi_{2}\left(a_{x}\right), \varphi_{3}\left(a_{x}\right) \ldots$, so resultiert schließlich die Darstellungsform 


$$
\left.\begin{array}{r}
-97- \\
w_{x}=\varphi\left(a_{x}\right)-\varepsilon(1) \varphi_{1}\left(a_{x}\right)+\frac{1}{1.2} \varepsilon(2) \varphi_{2}\left(a_{x}\right) \\
\quad-\frac{1}{1.2 .3} \varepsilon(3) \varphi_{3}\left(a_{x}\right)+\cdots
\end{array}\right\}
$$

wo nunmehr die Abhängigkeit des Funktionswertes $w_{x}$ von $a_{x}$ auch äußerlich hervortritt.

Hiernach bedarf man zur Darstellung der $z_{x}$ oder $w_{x}$ in ihrer Abhängigkeit von den $a_{*}$ lediglich der Mittelwertpotenzen $\varepsilon(1), \varepsilon(2), \varepsilon(3)$..., die auf Grund der gegebenen Beobachtungsreihe berechnet werden können. Die Mittelwerte $\varepsilon_{1}, \varepsilon_{2}, \varepsilon_{3} \ldots$ sind daher als die independenten (ron der Annahme einer Funktionsform unabhängigen) Parameter der Beobachtungsreihe zu betrachten und unmittelbar der Verwertung der Beobachtungsergebnisse zugrunde zu legen.

Für eine aus $\nu$ Gliedern $a_{1}, a_{2}, a_{3} \ldots a_{v}$ bestehende Beobachtungsreihe sind je $v$ Mittelwertpotenzen durch eine lineare Relation miteinander verknüpft. Man kann beispielsweise auf Grund der Gleichungen

$$
\begin{aligned}
& m \quad=z_{1} \quad+z_{2} \quad+\cdots z_{v} \\
& m \varepsilon(1)=z_{1}\left(a_{1}-a_{0}\right)+z_{2}\left(a_{2}-a_{0}\right)+\cdots z_{v}\left(a_{v}-a_{0}\right) \\
& m \varepsilon(2)=z_{1}\left(a_{1}-a_{0}\right)^{2}+z_{2}\left(a_{2}-a_{0}\right)^{2}+\cdots z_{\nu}\left(a_{\imath}-a_{0}\right)^{2} \\
& m \varepsilon(\nu-1)=z_{1}\left(a_{1}-a_{0}\right)^{v-1}+z_{2}\left(a_{2}-a_{0}\right)^{v-1}+\cdots z_{v}\left(a_{v}-a_{0}\right)^{r-1} \\
& m \varepsilon(v)=z_{1}\left(a_{1}-a_{0}\right)^{v}+z_{2}\left(a_{2}-a_{0}\right)^{v}+\cdots z_{v}\left(a_{\nu}-a_{0}\right)^{v}
\end{aligned}
$$

$\varepsilon(\boldsymbol{v})$ durch $\varepsilon(1), \varepsilon(2) \ldots \varepsilon(\nu-1)$ ausdrücken. Es kommen darum zur Bestimmung der Beobachtungsreihe bloß $v-1$ Mittelwerte in Betracht.

Denkt man sich die Beobachtungsreihe durch Zusammennehmen benachbarter Glieder auf eine kleinere Gliederanzabl reduziert, so ist auch eine entsprechend kleinere Anzahl von Mittelwerten zur Bestimmung der reduzierten Beobachtungsreihe ausreichend. Die reduzierte Form ist als eine näherungsweise gültige Darstellung der ursprünglichen Form zu betrachten. In gleicher Weise ist darum auch die entsprechend kleinere Anzahl von Mittel- 
werten als eine näherungsweise gültige Bestimmung der ursprünglich gegebenen Beobachtungsreihe anzusehen. Man wird dabei stets die aufeinanderfolgenden Mittelwerte 1., 2., 3. Ordnung usw. wählen, weil die Berechnung eines Mittelwertes höherer Ordnung die vorherige Berechnung der Mittelwerte niederer Ordnung voraussetzt.

Die a us $\nu$ Gliedern $a_{1}, a_{2}, a_{3} \ldots a_{v}$ bestehende Beobachtungsreihe wird somit durch $\nu-1$ Mittelwerte $\varepsilon_{1}, \varepsilon_{2}$, $\varepsilon_{3} \ldots \varepsilon_{v-1}$ vollständig und durch eine geringere Anzahl von $\mu$ Mittelwerten $\varepsilon_{1}, \varepsilon_{2}, \varepsilon_{3} \ldots \varepsilon_{\mu}(\mu<v-1)$ näherungs weise bestimmt.

Die Mittelwerte besitzen allgemein gültige Eigenschaften, die ich in meiner "Theorie der Kollektivgegenstände" (1902) abgeleitet habe. Insbesondere gelten folgende Sätze:

Sind $\varepsilon_{1}, \varepsilon_{2}, \varepsilon_{3}, \varepsilon_{4} \ldots$ die a uf den beliebigen Ausgangs wert $a_{0}$ bezogenen Mittelwerteder $z_{1}$ Größen $a_{1}, z_{2}$ Größen $a_{2}, z_{3}$ Größen $a_{3}, \ldots z_{v}$ Größen $a_{v}$, so ist

$$
\begin{aligned}
& 0<\varepsilon_{2}<\varepsilon_{4}<\varepsilon_{6}<\ldots \\
& \varepsilon_{1}^{2}<\varepsilon_{2}^{2} ; \quad \varepsilon_{3}^{2}<\varepsilon_{4}^{2} ; \quad \varepsilon_{5}^{2}<\varepsilon_{6}^{2} ; \ldots
\end{aligned}
$$

oder allgemein für $\varkappa=1,2,3 \ldots$

$$
\varepsilon_{2 x}<\varepsilon_{2 x+2} ; \varepsilon_{2 x-1}^{2}<\varepsilon_{2 x}^{2} . ., . .
$$

Es ist ferner für die Mittelwertpotenzen

$$
\begin{gathered}
\varepsilon(2) \varepsilon(4)>\varepsilon(3)^{2} ; \varepsilon(4) \varepsilon(6)>\varepsilon(5)^{2} ; \ldots \\
\frac{\varepsilon(2)}{1}<\frac{\varepsilon(4)}{\varepsilon(2)}<\frac{\varepsilon(6)}{\varepsilon(4)}<\cdots
\end{gathered}
$$

und allgemein für $x=1,2,3 \ldots ; \lambda=1,2,3 \ldots ; \mu=0$, $1,2 \ldots \lambda-1$

$$
\varepsilon(2 x) \cdot \varepsilon(2 x+2 \lambda)>\varepsilon(2 x+\lambda+\mu) \cdot \varepsilon(2 x+\lambda-\mu)
$$

Weiterhin besteht für $x=1,2,3 \ldots ; \lambda=1,2,3 \ldots$, wenn $\varkappa$ von $\lambda$ verschieden ist, die Ungleichung

$$
\left[\varepsilon(2 x)-\varepsilon(x)^{2}\right]\left[\varepsilon(2 \lambda)-\varepsilon(\lambda)^{2}\right]>[\varepsilon(x+\lambda)-\varepsilon(x) \varepsilon(\lambda)]^{2}
$$

Diese Bestimmungen bleiben in Geltung bei jedweder Verteilung der Werte $z_{x}$ auf die zugehörigen Intervalle. Werden 
aber die $z$-Werte stetig über die Intervalle ausgebreitet und zeigt es sich alsdann, daß sie mit zunehmender Entfernung vom Ausgangswerte $a_{0}$ abnehmen oder wenigstens nicht wachsen, so ist nach einer Bemerkung von Gauss in der Theoria combinationis

und allgemein ${ }^{1}$ )

$$
\check{5} \varepsilon(4) \geqq 9 \varepsilon(2)^{2} \quad \text {. . . . . . . . }
$$

$$
\varepsilon(2 x) \geqq \frac{3^{x}}{2 x+1} \varepsilon(2)^{x} ; \varepsilon(4 x) \geqq \frac{(2 x+1)^{2}}{4 x+1} \varepsilon(2 x)^{2}
$$

Entspricht die Verteilung der $z$ dem gewöhnlichen Fehlergesetze, so ist

$$
\left.\begin{array}{c}
\varepsilon(4)=3 \varepsilon(2)^{2} \\
\varepsilon(2)=1.3 .5 \ldots(2 \varkappa-1) \cdot \varepsilon(2)^{x}
\end{array}\right\} .
$$

Als Ausgangswert $a_{0}$ kann jeder beliebige Wert gewählt werden, und man kann überdies von einem anfänglich gewählten Werte zu einem anderen nach Belieben übergehen. Es empfiehlt sich aber, das arithmetische Mittel $b$ der beobachteten Werte als Ausgangswert zu benutzen. Es wird durch die Gleichung

$$
m b=z_{1} a_{1}+z_{2} a_{2}+z_{3} a_{3}+\cdots+z_{v} a_{v} .
$$

definiert. Es ist alsdann, wenn $a_{*}$ den ursprünglich gewählten Ausgangswert angibt und $\eta_{1}, \eta_{2}, \eta_{3} \ldots$ die auf $a_{*}$ bezogenen Mittelwerte bezeichnen,

$$
\begin{aligned}
b= & a_{x}+\eta_{1} ; \varepsilon=0 \\
\varepsilon(2) & =\eta(2)-\eta(1)^{2} \\
\varepsilon(3) & =\eta(3)-3 \eta(2) \eta(1)+2 \eta(1)^{3} \\
\varepsilon(4)= & \eta(4)-4 \eta(3) \eta(1)+6 \eta(2) \eta(1)^{2}-3 \eta(1)^{4} \\
\cdot . & \cdot \cdot \cdot \cdot \cdot \cdot \cdot \cdot \cdot \cdot \cdot \cdot \cdot \cdot \cdot \cdot \\
\varepsilon(\varrho)= & \eta(\varrho)-\frac{\varrho}{1} \eta(\varrho-1) \eta(1) \\
& \left.\quad+\frac{\varrho(\varrho-1)}{1.2} \eta(\varrho-2) \eta(1)^{2}-\cdots \pm(\varrho-1) \eta(1)^{?}\right)
\end{aligned}
$$

Schließlich ist $\mathrm{zu}$ beachten, daß die empirisch bestimmten Häufigkeitswerte $z_{1}, z_{2}, z_{3} \ldots z_{v}$ und ebenso die relativen Häufig-

1) Krüger, Über einen Satz der Theoria combinationis; Göttinger Nachrichten 1897 , s. 147. 
keitswerte $w_{1}, w_{2}, w_{3} \ldots w_{v}$ Repräsentanten ganzer Mannigfaltigkeiten von Werten sind, die bei unbegrenzt wiederholter Ausführung der Beobachtungsreihe auftreten würden. Es sind demzufolge auch die Mittelwertpotenzen $\varepsilon(1), \varepsilon(2), \varepsilon(3) \ldots$ und die Mittelwerte $\varepsilon_{1}, \varepsilon_{2}, \varepsilon_{3} \ldots$ als Repräsentanten entsprechender Mannigfaltigkeiten żu betrachten.

Um diese Mannigfaltigkeiten zu bestimmen, ersetze ich der Einfachheit wegen $\left(a_{1}-a_{0}\right)^{9},\left(a_{2}-a_{0}\right)^{\circ}, \ldots\left(a_{v}-a_{0}\right)^{\circ}$ oder, wenn $b$ als Ausgangswert dient, $\left(a_{1}-b\right)^{?},\left(a_{2}-b\right)^{?}, \ldots\left(a_{v}-b\right)^{\text {? }}$ durch $\alpha_{1}, \alpha_{2} \ldots \alpha_{r}$, so daß

$$
\varepsilon(\varrho)=w_{1} \alpha_{1}+w_{2} \alpha_{2}+\cdots+w_{v} \alpha_{v} . . .
$$

die aus $m=z_{1}+z_{2}+\cdots+z_{v}$ Beobachtungen abgeleitete Mittelwertpotenz angibt. Ich bezeichne ferner die relative Häufigkeit für das Auftreten der Anzahlen $z_{1}, z_{2} \ldots z_{v}$ bei der unbegrenzten Wiederholung der $m$ Beobachtungen durch $W\left(z_{1}, z_{2} \ldots z_{v}\right)$. Es ist nun, wenn die Summation über alle möglichen Werte $z_{1}, z_{2} \ldots z_{\nu}$, deren Summe gleich $m$ ist, durch $\Sigma^{\prime}$ angedeutet wird, für jedes $x=1,2, \ldots \nu$ und für jedes von $x$ verschiedene $\lambda^{1}$ )

$$
\left.\begin{array}{l}
\Sigma z_{x} W\left(z_{1} \ldots z_{v}\right)=m w_{x} \\
\Sigma z_{x}^{2} W\left(z_{1} \ldots z_{v}\right)=m(m-1) w_{x}^{2}+m w_{x} \\
\Sigma z_{x} z_{\lambda} W\left(z_{1} \ldots z_{\nu}\right)=m(m-1) w_{x} w_{\lambda}
\end{array}\right\} .
$$

Demzufolge wird das arithmetische Mittel aller Werte, die bei unbegrenzter Wiederholung der $m$ Beobachtungen an Stelle des tatsächlich in (66) vorliegenden Wertes treten würden, bestimmt durch

$$
\left.\begin{array}{l}
\frac{1}{m} \Sigma\left\{z_{1} \alpha_{1}+z_{2} \alpha_{2}+\cdots z_{v} \alpha_{v}\right\} W\left(z_{1} \ldots z_{v}\right) \\
=w_{1} \alpha_{1}+w_{2} \alpha_{2}+\cdots+w_{v} \alpha_{v}=\varepsilon(\varrho)
\end{array}\right\} .
$$

Man erhält ferner für die Quadrate der Abweichungen der Einzelwerte vou diesem arithmetischen Mittel

1) Die Ableitung der Summen gebe ich in der Abhandlung: „Die Bestimmung der Abhängigkeit zwischen den Merkmalen eines Gegenstandes". Berichte d. Sächs. Ges. d. Wiss. (Math.-phys. Klasse) '1905, S. 23,24 . 


$$
\begin{aligned}
\sum & -101- \\
= & \left.\frac{1}{m}\left(z_{1} \alpha_{1}+\cdots+z_{v} \alpha_{v}\right)-\varepsilon(\varrho)\right\}^{l^{2}} W\left(z_{1} \ldots z_{v}\right) \\
= & \frac{1}{m^{2}} \Sigma\left(z_{1} \alpha_{1}+\cdots+z_{2} \alpha_{v}\right)^{2} W\left(z_{1} \ldots z_{v}\right)-\varepsilon(\varrho)^{2} \\
= & \frac{m(m-1)}{m^{2}}\left(w_{1} \alpha_{1}+\cdots+w_{v} \alpha_{v}\right)^{2} \\
\quad & \quad+\frac{1}{m}\left(w_{1} \alpha_{1}^{2}+\cdots+w_{v} \alpha_{v}^{2}\right)-\varepsilon(\varrho)^{2} \\
= & \frac{1}{m}\left\{\varepsilon(2 \varrho)-\varepsilon(\varrho)^{2}\right\}
\end{aligned}
$$

wo

$$
\varepsilon(2 \varrho)=w_{1} \alpha_{1}^{2}+\cdots+w_{v} \alpha_{v}^{2} .
$$

Demgemäß repräsentiert der Wert $\varepsilon(\varrho)$ die durch das arithmetische Mittel und die mittlere quadratische Abweichung

$$
\varepsilon(\varrho) \pm \sqrt{\left\{\frac{1}{m}\left[\varepsilon(2 \varrho)-\varepsilon(\varrho)^{2}\right]\right\} . .} .
$$

charakterisierte Mannigfaltigkeit von Werten.

Die hier entwickelte Methode, die Mittelwerte $\varepsilon_{1}, \varepsilon_{2}, \varepsilon_{3} \ldots$ zur Bestimmung der Beobachtungsreibe zu benutzen, führt bei Beschränkung auf die Mittelwerte erster und zweiter Ordnung zum Gaussschen, in der Theoria combinationis angegebenen Verfahren zurück, die Beobachtungsreihe durch das arithmetische Mittel und den mittleren Fehler zu charakterisieren. Denn der Mittelwert erster Ordnung bestimint nach (65) das arithmetische Mittel, und der auf das arithmetische Mittel als Ausgangswert bezogene Mittelwert zweiter Ordnung ist nichts anderes als der Gausssche mittlere Fehler. In entsprechender Weise reduziert sich die Brunssche Reihe (48), wenn man beim ersten Gliede stehen bleibt, auf das Gausssche Fehlergesetz, sofern dieses Gesetz (was in der Regel der Fall sein wird) bei der Anwendung jener Reihe zugrunde gelegt wird. Das Prinzip, die Mittelwerte $\varepsilon_{1}, \varepsilon_{2}, \varepsilon_{3} \ldots$ als independente Parameter der Beobachtungsreihe zu verwenden, tritt somit der Brunsschen Reihe in ähnlicher Weise gegenüber, wie das Gausssche Prinzip des mittleren Fehlers dem Gaussschen Fehlergesetze. 
Mit Hilfe der Mittelwerte kann nun die Aufgabe, die Fechner unter Anwendung der psychophysischen Maßmethoden in den Elementen der Psychophysik behandelt hat, von neuem in Angriff genommen werden: man kann durch die Berechnung von absoluten Schwellen und Unterschiedsschwellen die Empfindlichkeit gegen Reize und Reizunterschiede messend verfolgen, um die Abhängigkeit zwischen Empfindungsintensität und Reizstärke zu bestimmen und hieraus im Sinne Fechners unter Anlehnung an das psychophysische Grundgesetz zu Maßbestimmungen in Gebiete der Bewußtseinserscheinungen zu gelangen.

Wir haben aber durch die grundlegenden Erörterungen des ersten Abschnittes erkannt, daß die Empfindungen und die Bewußtseinsinhalte überhaupt vierfach bedingt sind, und daß demgemäß auch die Aufgabe der experimentellen Psychologie in der dort (S. 15) angegebenen Weise als eine vierfache zu bezeichnen ist, während $\mathrm{Fechner}$, von naturphilosophischen Ideen beherrscht, eine mathematische Funktionsbeziehung zwischen Reizstärke und Empfindungsintensität voraussetzt und zur Prüfung derselben die Abhängigkeit zwischen Reiz und Empfindung untersucht. Darum ist der in den psychophysischen Maßmethoden zur Geltung gekommene Standpunkt Fechners durch einen solchen zu ersetzen, der die Verwendbarkeit von $\mathrm{Ma}$ b und Zahl bei den Untersuchungen der experimentellen Psychologie in ihrem vollen Umfange übersehen läßt.

$\mathrm{Zu}$ diesem Zwecke ist an die von Fechner entwickelte Auffassung des psychischen Maßes anzuknüpfen. 
Fünfter Abschnitt.

\section{Das psychische Maß.}

\section{Die durch Fechner begründete Auffassungsweise des psychischen Maßes.}

Das Maß der Empfindlichkeit, das durch die psychophysischen Maßmethoden vermittelt wird, ist, wie $\mathrm{Fechner}^{1}$ ) hervorhebt, noch kein Maß der Empfindung. "Wir messen dabei in der Tat nicht die Empfindung, sondern nur die Reize oder den Unterschied der Reize, welche eine gleich große Empfindung oder einen gleich großen Unterschied der Empfindung bewirken, und es fragt sich also noch, ob und inwiefern das $\mathrm{Ma}$ der Empfindung selbst und des Geistigen überhaupt möglich sei."

Für unmöglich kann es Fechner nicht halten. Er sagt vielmehr: "Von vornherein und im allgemeinen kann nicht bestritten werden, daß das Geistige überhaupt quantitativen Verbältnissen unterliegt. Denn nicht nur läßt sich von einer größeren und geringeren Stärke von Empfindungen sprechen, es gibt auch eine verschiedene Stärke von Trieben, es gibt größere und geringere Grade der Aufmerksamkeit, der Lebhaftigkeit von Erinnerungen und Phantasiebildern, der Helligkeit des Bewußtseins im ganzen, wie der Intensität einzelner Gedanken." Dem fügt er allerdings hinzu: „Zunächst und unmittelbar haben wir nur ein Urteil über ein Mehr oder Weniger oder ein Gleich in allen diesen Beziehungen, nicht über ein Wievielmal, was zu einem wahren Maße erforderlich wird, und welches zu gewinnen es gelten wird." Wir können beispielsweise sagen, daß von zwei Empfindungen die eine stärker ist als die andere, zum Maße der Empfindung gehört aber, daß wir sagen können, die eine Empfindung ist zweimal, dreimal, $n$ mal so stark wie die audere.

Dies wird nun nach der Ansicht Fechners durch die Funktionsbeziehung zwischen Leib und Seele möglich gemacht.

1) Elemente der Psychophysik 1, 54 ff. 
Besteht nämlich eine solche Beziehung, so kann man im physischen Gebiete Messungen ausführen und hierdurch zugleich psychische Größen bestimmen. Insbesondere lassen sich auf Grund der Funktionsbeziehung zwischen Reiz und Empfindung Reizzuwïchse bèstimmen, denen nach der Annahme Fechners gleiche Empfindungszuwüchse zur Seite stehen, so dals ans der Anzahl der variablen Reizzuwüchse, die nötig sind, um eine Empfindung von bestimmter Stärke zu erzeugen, die Anzahl gleicher Empfindungszuwüchse und somit die Größe der Empfindung selbst erschlossen werden kann.

Wäre demgemäß $y$ die zu bestimmende Empfindungsstärke und $x$ die zugehörige meßbare Reizgröße, so könnte man $x$ in die Summe

$$
x_{1}+\left(x_{2}-x_{1}\right)+\left(x_{3}-x_{2}\right)+\cdots+\left(x_{n}-x_{n-1}\right)+\left(x-x_{n}\right)
$$

zerlegen und $x_{1}, x_{2}, x_{3} \ldots x_{n}$ so bestimmen, daß zu $x_{1}$ die Empfindung mit verschwindender Stärke, zu $x_{2}-x_{1}, x_{3}-x_{2} \ldots x-x_{n}$ aber immer der nämliche Empfindungszuwachs gehörte. Wählte man diesen konstanten Empfindungszuwachs als Einheit, so wäre folglich $y$ als eine aus $n$ Einheiten bestehende Empfindung anzusehen.

Das Maß der Empfindung kommt demnach, wie Fechner selbst sagt, darauf hinaus, „jede Empfindung in gleiche Abteilungen, d. i. die gleichen Inkremente, aus denen sie rom Nullzustande an erwächst, zu zerlegen und die Zahl dieser gleichen Abteilungen als wie durch die Zolle eines Mabstabes durch die Zahl der zugehörigen variablen Reizzuw üchse bestimmt zu denken, welche die gleichen Empfindungszuwüchse herrorzubringen imstande sind".

Die Zugehörigkeit variabler, und zwar bei Geltung des Weberschen Gesetzes relativ konstanter Reizzuwüchse zu gleichen Empfindungszuwüchsen betrachtete Fechner als eine letzte, das psychophysische Grundgesetz bedingende Tatsache, in der die Wechselwirkung zwischen Physischem und Psychischem zum Ausdruck kommt. Dieser psychophysischen Deutung des Weberschen Gesetzes stellte G. E. Müller in seiner "Grundlegung der Psychophysik" eine physiologische Deutung gegenüber, nach welcher die der Empfindung unmittelbar zur Seite stehende Erregung der Nervensubstanz der Empfindung propor- 
tional gehe und die wechselnde Größe des äußeren Reizvorganges durch eine Umänderung auf dem Wege nach dem Zentralorgane bedingt werde. Wundt hingegen hat dem Weberschen Gesetze eine psychologische Deutung gegeben, indem er es aus den psychischen Vorgängen, die bei der Empfindung wirksam sind, ableitete und als einen Spezialfall des allgemeinen Gesetzes der Relativität der Bewußtseinszustände auffaßte.

Diese Deutungen haben jedoch auf das Maßprinzip der Empfindung als solches keinen Einfluß. Denn die Funktionsbeziehung, die allein als Unterlage für das Empfindungsmaß Fechners in Betracht kommt, bleibt bei jeder Auffassungsweise bestehen, so daß in jedem Falle die Empfindung als eine in Teile zerlegbare und aus Teilen zusammensetzbare Größe auffaßbar erscheint.

Von anderer Seite (durch v. Kries, F. A. Müller, Ulrici, Zeller) wurde dies allerdings bestritten, jedoch ohne daß eine positive Auffassungsweise entwickelt worden wäre, die als Ausgangspunkt für eine auf das Fechnersche Maßprinzip verzichtende Ausführung der psychophysischen Untersuchungen hätte dienen können. Darum konnte Fechner diese Gegner in seiner "Revision der Hauptpunkte der Psychophysik" (1882) auf die tatsächlich vorliegenden Ergebnisse psychophysischer Untersuchungen hinweisen.

So kam es, daß die von Fechner entwickelte Idee, auf die funktionelle Abhängigkeit zwischen Reiz und Empfindung das Maß der Empfindung zu gründen, zur Anerkennung gelangte. Nur die Frage, ob die gleich merklichen Empfindungsunterschiede auch immer gleich große oder vielmehr je nach Umständen verschieden große Unterschiede seien, und die hierdurch bedingte praktische Ausgestaltung des psychischen Maßes blieb unentschieden und der Diskussion unterworfen.

Demgemäß wird z. B. in den Untersuchungen Külpes ${ }^{1}$ ) , Über das Verhältnis der eben merklichen zu den übermerklichen Unterschieden" nicht danach gefragt, ob und inwieweit überhaupt von der Größe eines Empfindungsunterschiedes geredet werden könne. Es handelt sich vielmehr nur darum, auf experimentellem Wege eine Entscheidung darüber herbeizuführen, ob einer Veränderung des

1) Psychologen-Kongre $B$ in Paris 1900, S. 1, 2, 6. 
eben merklichen Reizunterschiedes „auch entsprechende Veränderungen des Empfindungsunterschiedes gegenübergestellt werden müssen". Während es Psychologen gibt, „die einen eben merklichen Unterschied als eine konstante Größe auffassen", während insbesondere Stumpf und Meinong diese Auffassungsweise zwar nicht als a priori notwendig, aber doch als annehmbar betrachten, findet $\mathrm{K} \ddot{\mathrm{l}} \mathrm{lpe}$ seinerseits die Annahme geboten, „daß die eben merklichen Unterschiede mit der Intensität der sie begrenzenden Empfindungen wachsen". Der eben merkliche Empfindungsunterschied ist dann allerdings nicht mehr als Maßeinheit brauchbar; das psychophysische Formelsystem Fechners wird jedoch hierdurch nicht, wie Külpe meint, hinfällig, sondern nur in seiner Form kompliziert, da in den Empfindungsunterschieden nunmehr aus Einheiten zusammengesetzte Größen an Stelle der Einheitsgrößse selbst vorliegen.

Behält aber Fechners Idee des psychischen Maßes und der funktionellen Abhängigkeit zwischen Reiz und Empfindung Geltung, so bleibt auch das Interesse an der Feststellung der Abhängigkeit zwischen Reiz und Empfindung im Vordergrunde, olıne daß eine einheitliche Auffassung des Gesamtgebietes der experimentellen Psychologie und eine ihren Bedürfnissen genügende Ausbildung der Maßmethoden möglich wird.

Dies zeigt sich bei $\mathrm{Fechner}$ selbst, indem er seine, einem anderen Teile der experimentellen Psychologie angehörenden Untersuchungen über das $\mathrm{Maß}$ ästhetischen Wohlgefallens und Mißfallens den psychophysischen Maßbestimmungen nicht anzugliedern vermochte. Es zeigt sich ferner darin, daß die Untersuchungen der Zeitverhältnisse und des Verlaufs der seelischen Vorgänge, durch welche Wundt schon in den "Beiträgen zur Theorie der Sinneswahrnehmung" (1862) und in den „Vorlesungen über die Menschen- und Tierseele" (1863) den Kreis der psychophysischen Untersuchungen im engeren Sinne des Wortes durchbrochen hat, nicht eine erweiterte Auffassung und modifizierte Behandlung der traditionellen psychophysischen Maßmethoden veranlaßten, obwohl sie eine grundlegende Bedeutung besitzen. Sie führen nämlich die Erforschung der in der Persönlichkeit des Beobachters überhaupt begründeten subjektiven Faktoren im Anschluß an die von Bessel entdeckte persönliche Gleichung der Astronomen in die Psychologie ein und erschließen so im 
Verein mit den auf die Ungenauigkeit der Sinneswahrnehmung gerichteten psychophysischen Messungen den Zugang zu dem gesamten, vom psychologischen Experiment beherrschten Forschungsgebiete.

Der Grund für diese Einseitigkeit liegt offenbar in der Ansicht, daß man entweder eine mathematisch darstellbare Funktionsbeziehung im Sinne Fechners annehmen oder auf Maßbestimmungen im Gebiete des Bewußtseins überhaupt verzichten müsse. Daß aber hier ein Irrtum vorliegt, zeigen folgende Bemerkungen.

\section{Orduen und Messen.}

Nach den eigenen Angaben Fechners können wir bei der Beurteilung von Empfindungen zunächst nur ein Mehr oder Weniger oder ein Gleich, nicht ein Wievielmal feststellen. Das Mehr oder Weniger ist aber nicht etwa ein Mehr oder Weniger an unterscheidbaren Teilen, sondern ein Stärker oder Schwächer, ein Ähnlicher oder Unähnlicher, das einer Umdeutung in ein Wievielmal, soweit die Empfindungen als solche in Betracht gezogen werden, in keiner Weise fähig ist, wohl aber ein Ordnen nach Stärkegraden oder sonstigen, verwandtschaftlichen und gegensätzlichen Beziehungen möglich macht.

Es ist doch wohl unmittelbar einleuchtend, daß beispielsweise durch "Hellgrau" die größere Ähnlichkeit mit Weiß oder der größere Gegensatz zu Schwarz angedeutet wird, und daß die Ähnlichkeit oder der Gegensatz in der kleineren oder größeren Anzahl unterscheidbarer Zwischenglieder zwischen Grau einerseits und Weiß oder Schwarz andererseits seinen Grund findet. Denn sobald die Zwischenglieder fehlen, wie z. B. zwischen einer Gesichts - und Gehörsempfindung, besteht bloß eine beziehungslose Verschiedenheit, ohne daß ein Ähnlichkeitsgrad angebbar wäre. - Es wird hingegen niemand, der nicht von vornherein die Feststellung quantitativer Bestimmungen beabsichtigt, darauf verfallen, mit Plateau (S. 76) das Kennzeichen der Empfindung „Hellgrau ${ }^{4}$ darin zu suchen, daß „die Stärke der in uns erregten Empfindung größer als die Hälfte derjenigen Empfindungsstärke ist, die ein weißer Gegenstand unter den nämlichen Beleuchtungsverhältnissen erregen würde". Die verschiedenen Grau-Empfindungen sind ja bloße Unterscheidungen, die sich voneinander ab- 
stufen und darum in eine Reibe ordnen lassen, nicht aber als Quanta, die aus Einheiten zusammengesetzt und in diese Einheiten zerlegbar wären, sich darbieten.

Darum finden die unterscheidbaren Inhalte des Bewußtseins, die auf Grund ihrer verwandtschaftlichen und gegensätzlichen Beziehungen in Reihen geordnet werden können, in den Ordnungszahlen $1,2,3 \ldots$ ihre Urbilder. Wie nämlich die einzelnen Glieder der Zahlenreihe die vorangehenden von den nachfolgenden einesteils trennen und anderenteils zueinander in Beziehung setzen, in derselben Weise werden auch die Glieder einer Reihe von Bewußtseinsinbalten durch die Zwischenglieder getrennt und miteinander verknüpft. Dabei bleibt aber jedes Glied, was es ist: es läßt sich nicht durch Summen- oder Differenzbildung, nicht durch Vervielfachung oder Teilung aus anderen Gliedern ableiten.

Zur Veranschaulichung können die Schüler einer Klasse dienen. Denn auch da läßt sich nur von einem Mehr oder Weniger an Leistungen reden, ohne daß in einwandfreier Weise eine Einheitsleistung feststellbar wäre und nun angegeben werden könnte, wievielmal sie bei dem einen oder dem anderen Schüler sich vorfindet. Es ist aber in der Regel eine Rangordnung herstellbar. Dann kann man den letzten der Klasse mit der niedrigsten Intensitätsstufe einer Empfindungsreihe vergleichen und dem Ansteigen der Leistungen innerhalb der Klasse das Ansteigen der Intensitätsgrade innerhalb der Empfindungsreihe zur Seite stellen. Es ist ferner eine so enge Abstufung der Leistungen denkbar, daß die aufeinander folgenden Stufen nur noch eben unterscheidbar und somit den eben merklichen Empfindungsunterschieden vergleichbar sind. In diesem Falle sind je zwei aufeinander folgende Schüler ebenso wie je zwei aufeinander folgende Empfindungen in gleicher Weise, nämlich in eben merklicher Weise verschieden. Diese Verschiedenheit wird aber doch nun nicht zu einer Größe, welche die Zuwüchse darstellt, aus denen die beim ersten der Klasse vorhandene Höhe an Leistungen von dem beim letzten feststellbaren Tiefstande aus erwächst. Ebensowenig läßt sich die Empfindung, wie Fechner will, in gleiche Abteilungen, aus denen sie rom Nullzustande aus erwächst, zerlegen. Die vermeintlich existierenden "gleichen Abteilungen" sind auch nicht durch "Abteilungen, die mit dem Fortschreiten innerhalb der Reihe wachsen", ersetzbar. Es kann vielmehr lediglich die An- 
zahl der eben voneinander unterscheidbaren $Z$ wischenglieder als Kennzeichen für die Größe der Verschiedenheit dienen, ohne daß eine Entscheidung darüber möglich wäre oder auch nur in Frage käme, ob die $Z$ wischenstufen bei geringwertigen und bei vollwertigen Leistungen, bei niedrigen und bei hohen Intensitätsgraden gleich groß oder überhaupt miteinander vergleichbar seien. Vemgemäß gehört zu jeder Empfindung ebenso wie zu jedem Schüler eine Ordnungszahl, welche die Stelle innerhalb der Reihe markiert. Es kann aber gar nicht daran gedacht werden, die einzelne Empfindung oder den einzelnen Schüler durch eine Maßzahl zu bestimmen, welche die Anzahl der Einheiten, aus denen die Empfindungsstärken oder die Leistungen angeblich bestehen, angeben würde.

Diese Erkenntnis findet in folgendem Grundsatz ihren Ausdruck: Lassen sich Bewubtseinsinhalte nach einem der Abstufung fähigen Merkmale in cine Reihe eben merklich unterscheidbarer Glieder ordnen, so gehört zu jedem Bewußtseinsinhalt eine Ordnungsahl, welche die Stelle innerhalb der Reihe angibt. Die Differenz der Ordnungszahlen zweier Bewußtseinsinhalte der nämlichen Reihe bezeichnet alsdann die Anzahl der Zwischenglieder, die von dem einen Gliede der Reihe zu dem anderen führen.

Die größere oder kleinere Anzahl der Zwischenglieder tritt im Bewußtsein in der größeren oder kleineren Ähnlichkeit der Bewußtseinsinhalte zutage, ohne daß etwa dem Bemerken der Ähnlichkeit das Erfassen der einzelnen Zwischenglieder vorhergehen müßte. Durch die Zwischenglieder wird zugleich die Größe der benachbarten Unterschiede bedingt. Die liernach bestehende Möglichkeit, von einem Mehr oder Minder des Unterschiedes zu reden, darf aber nicht dazu verleiten, die Bewußtseinsinbalte selbst als Quanta aufzufassen, die in Einheiten zerlegt und aus Einheiten zusammengesetzt werden können.

Solche meßbare und überdies stetig veränderliche Quanta sind hingegen die objektiven Zustände und Vorgänge, die den Bewußtseinsinhalten zagrunde liegen. Es treten darum den Ordnungsahlen der Bewubtseinsinhalte die Mabzahlen der zugrunde liegenden objektiven Zustände und Vorgänge zur Seite. Diese Zuordnung von Ordnungszahlen und 
Maßzahlen für das Gebiet der Empfindungen in ihrer Gesetzmäßigkeit zu erforschen, ist die (von der entbehrlichen Zutat des psychophysischen Grundgesetzes befreite) Aufgabe, die Fechner in den Elementen der Psychophysik gestellt hat. Ebendieselbe Zuordnung ist aber überall vorhanden, wo überhaupt einer im Bewußtsein vollzogenen Unterscheidung meBbare objektive $\mathrm{Zu}$ stände oder Vorgänge zur Seite stehen. Sie unter Berücksichtigung aller in Betracht kommender, bei der Erörterung der Bewußtseinsinhalte ( $\$ 2$, S. 14) geltend gemachter Faktoren festzustellen und zur Untersuchung der Bewußtseinsinhalte selbst zu benutzen, ist die Aufgabe der experimentellen Psychologie, soweit sie nicht auf bloß qualitative, sondern auf quantitative, die Verwendung von Maß und Zahl in Anspruch nehmende Bestimmungen ausgeht.

Hierbei ist zu einer Einschränkung der Untersuchung auf die Beziehung zwischen Reiz und Empfindung im Sinne der Psychophysik Fechners kein Grund vorhanden. Man wird sich vielmehr nicht minder die Aufgabe stellen, beispielsweise die der Ausführung von Reaktionen zugrunde liegenden objektiven Zeiten zu messen oder die mit dem höchsten Grade des Wohlgefallens oder Mißfallens behafteten Verhältnisse an geometrischen Figuren festzustellen. Es gliedern sich somit die von Wundt in die Psychologie eingeführten psychischen Zeitmessungen und die von Fechner begründete experimentelle Ästhetik der psychophysischen Untersuchung von Reiz und Empfindung ohne weiteres an.

Aber auch die psychophysische Grundaufgabe, die in der Ableitung einer normalen, durch alle wechselnden und störenden Finflüsse hindurch sich behauptenden gesetzmäßigen Zuordnung von Reiz und Empfindung besteht, erleidet keine Einbuße. Es ist nur im Auge zu behalten, daß nicht eine die Größe der Empfindung angeblich bestimmende Maßzabl, sondern eine die Stelle der Empfindung in der Reihe eben merklicher Abstufungen bezeichnende Ordnungszahl in ihrer gesetzmäßigen Abhängigkeit von der Maßzahl des Reizes zu bestimmen ist.

Um diese Abhängigkeit darzustellen, mögen die Maßzahlen der Reize, die zu einer Reihe eben merklich abgestufter Empfindungen $e_{1}, e_{2}, e_{3} \ldots e_{n}$ gehören, als Punkte einer Geraden gedeutet werden. In dieser Geraden sollen sodann die den Empfin- 
dungen normalerweise zugehörenden Werte $r_{1}, r_{2}, r_{3} \ldots r_{n}$ samt den oberen und unteren Unterschiedsschwellen $r_{1}+i_{1}$ und $r_{1}-i_{1}, r_{2}+i_{2}$ und $r_{2}-i_{2}, r_{3}+i_{3}$ und $r_{3}-i_{3} \ldots r_{n}+i_{n}$ und $r_{n}-i_{n}$ bestimmt werden. Es ergeben sich so die Intervalle

$$
r_{1} \pm i_{1}, r_{2} \pm i_{2}, r_{3} \pm i_{3} \ldots r_{n} \pm i_{n},
$$

die unmittelbar aneinander grenzen, weil es sich um eben merklich verschiedene Empfindungen handelt. Sie finden in dem folgenden Linienschema ihre Veranschaulichung, wo die Annahme gemacht wird, daß die Intervalle mit wachsender Ordnungszahl sich vergrößern.

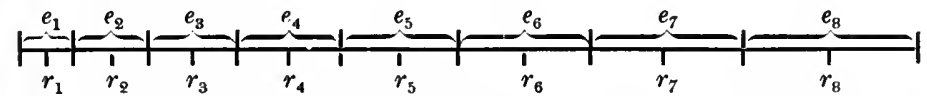

Gilt es nun, das die Zuordnung von Reiz und Empfindung beherrschende Gesetz zum Ausdruck zu bringen, so ist hierzu bloß nötig, die Unterschiedsschwellenwerte $i$ als Funktion der zugehörigen Reizwerte $r$ darzustellen. Denn die Funktion $i=\varphi(r)$ gibt zunächst den zu einem beliebigen Reize $r_{1}$ gehörigen Unterschiedsschwellenwert $i_{1}$. Da aber $r_{2}-i_{2}=r_{1}+i_{1}$, weil die obere Unterschiedsschwelle von $r_{1}$ mit der unteren Unterschiedsschwelle des eben merklich größeren Reizes $r_{2}$ zusammenfällt, so erhält man aus $r_{2}-\varphi\left(r_{2}\right)=r_{1}+\varphi\left(r_{1}\right)$ den Wert $r_{2}$; in entsprechender Weise aus $r_{3}-\varphi\left(r_{3}\right)=r_{2}+\varphi\left(r_{2}\right)$ den Wert $r_{3}$ usw.

Haben z. B. die Unterschiedsschwellen alle denselben Wert $i$, so ist

$$
\begin{aligned}
& r_{2}=r_{1}+2 i \\
& r_{3}=r_{2}+2 i=r_{1}+4 i \\
& r_{4}=r_{3}+2 i=r_{2}+4 i=r_{1}+6 i
\end{aligned}
$$

und allgemein für beliebige Ordnungszahlen $x$ und $\lambda$

so daß

$$
\begin{aligned}
& r_{\varkappa}=r_{1}+2(x-1) i \\
& r_{\lambda}=r_{1}+2(\lambda-1) i,
\end{aligned}
$$

$$
r_{*}-r_{\lambda}=2(x-\lambda) i \text {. }
$$

Die Differenzen der Ordnungszahlen für die Reihe eben merklich abgestufter Empfindungen sind somit den Differenzen der Maßwerte der zugehörigen Reize 
proportional, weun die Unterschiedsschwellenwerte konstant sind.

Gilt hingegen das Webersche Gesetz, so ist $i=c . r$ zu setzen, wo $c$ einen konstanten Wert bezeichnet. Man erhält alsdann, da.

und

$$
i_{1}=c . r_{1}, \quad i_{2}=c . r_{2}, \quad i_{3}=c . r_{3} \ldots
$$

$$
r_{1}+i_{1}=r_{2}-i_{2}, r_{2}+i_{2}=r_{3}-i_{3}, r_{3}+i_{3}=r_{4}-i_{4} \ldots,
$$

zunächst

$$
\begin{aligned}
& r_{1}+c r_{1}=r_{2}-c r_{2} \\
& r_{2}+c r_{2}=r_{3}-c r_{3} \\
& r_{3}+c r_{3}=r_{4}-c r_{4}
\end{aligned}
$$

und, wenn $(1+c):(1-c)=\gamma$ gesetzt wird,

$$
\begin{aligned}
& r_{2}=\gamma \cdot r_{1} \\
& r_{3}=\gamma \cdot r_{2}=\gamma^{2} \cdot r_{1} \\
& r_{4}=\gamma \cdot r_{3}=\gamma^{2} \cdot r_{2}=\gamma^{3} \cdot r_{1}
\end{aligned}
$$

Für beliebige Ordnungszahlen $x$ und $\lambda$ ist daher

so daß

$$
\begin{aligned}
& r_{x}=\gamma^{x-1} \cdot r_{1} \\
& r_{\lambda}=\gamma^{\lambda-1} \cdot r_{1},
\end{aligned}
$$

$$
r_{*}: r_{\lambda}=\gamma^{\varkappa-\lambda} \text { oder }(x-\lambda) \log \gamma=\log r_{x}-\log r_{\lambda}
$$

Die Differenzen der Ordnungszahlen für die Reihe eben merklich abgestufter Empfindungen sind demgemäß den Differenzen der Logarithmen von den Maßwerten der zugehörigen Reize proportional, wenn bei Gültigkeit des Weberschen Gesetzes die Unterschiedsschwellenwerte relativ konstant sind.

In der Formel (72) bietet sich das aus dem Weberschen Gesetze ableitbare Gesetz der Zuordnung von Reiz und Empfindung dar. Es setzt die Möglichkeit voraus, die Empfindungen in eine Reihe eben merklicher Abstufungen zu ordnen und den relativ konstanten Betrag der Unterschiedsschwelle zu ermitteln. Es stellt hingegen an die Empfindungen nicht den Anspruch, sich als meßbare Quanta darzubieten. Es leistet aber trotzdem alles, 
was überhaupt geleistet werden kann, indem es die Norm angibt, nach der bei relativer Konstanz der Unterschiedsschwelle die $\mathrm{Zu}-$ ordnung der eben merklich verschiedenen Empfindungen zu dem stetig ausgedehnten Reizgebiete zu vollziehen ist.

Soll nun eine Aufgabe aus dem Gebiete der experimentellen Psychologie unter Inanspruchnahme von Maß und Zahl bearbeitet werden, so kann es sich den vorstehenden Darlegungen gemä $B$ nur darum handeln, die Zusammengehörigkeit der Maßwerte objektiver Zustände und Vorgänge einerseits und gewisser subjektiver Auffassungen und Unterscheidungen andererseits festzustellen.

Dabei sind zwei Verfahrungsweisen möglich. Entweder kann man den objektiven Zustand oder Vorgang, der einer im Bewußtsein gegebenen Norm entspricht, herstellen und seinen Maßwert bestimmen, oder man kann feststellen, wie ein gegebener objektiver Zustand oder Vorgang subjektiv erfaßt und beurteilt wird. Die für das psychophysische Untersuchungsgebiet (S. 80) vollzogene Unterscheidung zwischen der Herstellung von Reizen, die einer aufgestellten Norm gemäß beurteilt werden, und der Beurteilung gegebener Reize hat somit in entsprechender Form für das ganze Gebiet der experimentellen Psychologie Geltung.

Die Zuordnung objektiver Maßwerte zu bestimmten Inhalten des Bewußtseins unterliegt aber verschiedenartigen Einflüssen, die sich im allgemeinen von Fall zu Fall ändern. Darum gilt es, den Grad der unter der Herrschaft solcher wechselnden Einflüsse sich behauptenden Abhängigkeit zu bestimmen. Es ist insbesondere festzustellen, ob überhaupt zu einem bestimmten Bewußtseinsinhalt normalerweise ein bestimmter Maßwert gehört.

Ist dies der Fall, so darf der Maßwert als Kennzeichen des Bewußtseinsinhaltes benutzt werden. Weil dies bei der Abhängigkeit zwischen Reiz und Empfindung zutrifft, so ist es möglich, der Empfindung den ihr normalerweise zugehörenden Reizwert zu substituieren und so an Stelle der verschiedenen, einem gegebenen Reizwerte bei wiederholter Beobachtung zukommenden Beurteilungen die zugehörigen Reize zu setzen und anzunehmen, daß diese Reize übereinstimmend beurteilt worden seien. In gleicher 


\section{- $114-$}

Weise kann in jedem Falle, wo eine normale Zugehörigkeit vorhanden ist, die wechselnde Auffassung eines und desselben Objektes durch die übereinstimmende Auffassung verschiedener Objekte er'setzt gedacht werden. Demnach ist der Erfolg einer Reihe von Beobachtungen stets in der Weise darstellbar, als wenn eine Anzahl verschiedener Objekte in übereinstimmender Weise erfaßt und beurteilt worden wäre. Die Beobachtungsreihe ist alsdann eine solche von Schema A (S. 83).

Liegt eine solche Beobachtungsreibe vor, so sieht man sich vor die Aufgabe gestellt, nicht etwa bloß die Bestätigung der normalen Zugehörigkeit eines objektiven Maßwertes zu einem subjektiven Bewußtseinsinhalte nachzuweisen, oder den Einfluß konstanter Fehler, die das Hervortreten der Zugehörigkeit hindern, aufzudecken; sondern insbesondere auch das in der ganzen Beobachtungsreihe hervortretende gesetzmäßige Wirken der Einflüsse, unter deren Herrschaft die einzelnen Werte sich ergeben haben, festzustellen. Zu diesem Zwecke ist der Typus der Beobachtungsreihe zu bestimmen.

Es ist sodann die Kenntnis des Typus zu benutzen, um aus der Veränderung desselben bei gleichzeitiger Änderung gewisser, die Beobachtungsreihe beherrschender Einflüsse einen $\mathrm{Zu}$ sammenhang zwischen diesen Einflüssen und dem ''y pus der Beobachtungsreibe zu ermitteln. Insbesondere ist der Einfluß des Reizwertes auf die Streuung der beobachteten Werte und die Unterschiedsschwelle als Maß dieses Einflusses zu bestimmen. - Es bietet sich demnach die Bestimmung der Unterschiedsschwelle nur als ein besonderer Fall des allgemeinen und bei allen Beobachtungsreihen der experimentellen Psychologie auftretenden Problems dar, das Wirken besonderer Einflüsse festzustellen und $\mathrm{zu}$ untersuchen. 
Sechster Abschnitt.

\section{Die Methoden der psychischen Abhängigkeits- bestimmung.}

\section{Die Bestimmung des Grades der Abhängigkeit.}

Zwischen einem objektiven Zustande oder Vorgange $a$ einerseits und einem Bewußtseinsinhalte $b$ andererseits besteht eine vollkommene Abhängigkeit, wenn das Auftreten des einen das Auftreten des anderen ausnahmslos einschließt oder ausschließt. Es ist ferner eine gewisse Abhängigkeit anzuerkennen, wenn wenigstens in der Regel $a$ und $b$ aneinander geknüpft sind oder einander ausschließen. Soll aber der tatsächlich vorhandene Grad der Abhängigkeit festgestellt werden, so ist durch eine hinreichend große Anzahl von Beobachtungen, die unter vergleichbaren Umständen anzustellen sind, die relative Häufigkeit oder Wahrscheinlichkeit des vereinten und gesonderten Auftretens von $a$ und $b$ zu ermitteln.

Hierbei bedingt es einen wesentlichen Unterschied, ob das Auftreten und Ausbleiben von $a$ oder $b$ beliebig herbeigeführt werden kann oder der Beeinflussung seitens des Beobachters sich entzieht. Bedeutet beispielsweise $a$ einen Reizwert und $b$ eine Empfindung, so kann man in der Regel so oft man will das Auftreten oder Ausbleiben der Reizwirkung und der Empfindung herbeiführen, um in dem.einen wie in dem anderen Falle das Vorhandensein oder Fehlen der Empfindung und des Reizes, um deren wechselweise Abhängigkeit es sich handelt, festzustellen. Bezeichnet hingegen $b$ ein Gefühl und $a$ den objektiv im Leibeszustande hervortretenden Gefühlsausdruck, so kann man im allgemeinen weder über $b$ noch über $a$ willkürlich verfügen, um ihren Zusammenhang in gleicher Weise wie bei Empfindung und Reiz zu untersuchen.

Vergleicht man die einzelnen Beobachtungen mit dem Ziehen einzelner Kugeln aus einer Urne, wo das Auftreten und das 


\section{$-116$}

Ausbleiben von $a$ durch ein der Kugel aufgeschriebenes Zeichen $a_{1}$ und $a_{2}$, das Auftreten und das Ausbleiben von $b$ in entsprechender Weise durch $b_{1}$ und $b_{2}$ angedeutet werden möge, so hat man sich demnach entweder die Kugeln in zwei Urnen verteilt zu denken, so daß die eine etwa alle Kugeln mit dem Zeichen $b_{1}$, die andere alle Kugeln mit dem Zeichen $b_{2}$ enthält, oder die Kugeln insgesamt in einer Urne vereinigt anzunehmen.

Sind nun zwei Urnen vorhanden, so kann man jede unabhängig von der anderen auf das Vorkommen von $a_{1}$ und $a_{2}$ untersuchen und das Ergebnis der Untersuchung in dem Schema

\begin{tabular}{c|l||l|l}
\multicolumn{2}{c||}{$b_{1}$} & \multicolumn{2}{c}{$b_{2}$} \\
\hline$a_{1}$ & $z_{11}$ & $a_{1}$ & $z_{21}$ \\
$a_{2}$ & $z_{12}$ & $a_{2}$ & $z_{22}$
\end{tabular}

zusammenstellen, wo $z_{11}$ und $z_{12}$ die Häufigkeit für das Auftreten und Ausbleiben von $a$ bei vorhandenem $b$ und $z_{21}$ und $z_{22}$ die entsprechenden Häufigkeiten bei fehlendem $b$ angeben. Es sind hier die Summen $z_{11}+z_{12}$ und $z_{21}+z_{22}$ als gleich groß vorauszusetzen, damit die Beobachtungen für $b_{1}$ und für $b_{2}$ miteinander vergleichbar seien. Ist jede Summe gleich $m$, so werden die Wahrscheinlichkeiten durch

$$
\left.\begin{array}{l}
w_{11}=z_{11}: m, \quad w_{12}=z_{12}: m, \quad w_{11}+w_{12}=1 \\
w_{21}=z_{21}: m, \quad w_{22}=z_{22}: m, \quad w_{21}+w_{22}=1
\end{array}\right\}
$$

bestimmt.

Gehören aber alle Kugeln einer einzigen Urne an, so lassen sich die Beobachtungen in dem Schema

\begin{tabular}{c||c|c} 
& $b_{1}$ & $b_{Z}$ \\
\hline \hline$a_{1}$ & $z_{11}$ & $z_{21}$ \\
$a_{2}$ & $z_{12}$ & $z_{22}$
\end{tabular}

zusammenfassen, wo die neben $a_{1}$ oder $a_{2}$ und unter $b_{1}$ oder $b_{2}$ stehende Zahl $z$ angibt, wie oft das Auftreten oder Ausbleiben von $a$ mit dem Auftreten oder Ausbleiben von $b$ zusammengetroffen ist. Wird die Gesamtzahl der Beobachtungen durch $m$ angegeben, so erhält man für die Wahrscheinlichkeiten die Werte 


$$
\left.\begin{array}{c}
-117- \\
w_{11}=z_{11}: m, \quad w_{21}=z_{21}: m \\
w_{12}=z_{12}: m, \quad w_{22}=z_{22}: m \\
w_{11}+w_{12}+w_{21}+w_{22}=1
\end{array}\right\} . . . .
$$

Es ist nun $a$ unabhängig von $b$, wenn das Auftreten und Ausbleiben von $a$ unabbängig von dem Auftreten und Ausbleiben von $b$ ist.

Es besteht demnach keine Abhängigkeit, wenn in $\mathrm{C}$ oder $\mathrm{C}_{1}$

Dann ist auch

$$
z_{11}: z_{12}=z_{21}: z_{22}
$$

$$
z_{11}: z_{21}=z_{12}: z_{22},
$$

so daß sich zugleich $b$ als unabhängig von $a$ erweist. Beide Proportionen führen zu der Gleichung

oder

$$
z_{11} z_{22}-z_{12} z_{21}=0
$$

$$
w_{11} w_{22}-w_{12} w_{21}=0 \text {. . . . . }
$$

Sind die Werte insbesondere dem Schema $C_{1}$ entnommen, so daß die Bestimmungen (73) in Kraft treten, so folgt hieraus durch Elimination von $w_{12}$ und $w_{22}$ oder von $w_{11}$ und $w_{21}$

$$
w_{11}-w_{21}=0 \text { oder } \quad w_{22}-w_{12}=0 .
$$

Man hat somit das Urteil über die Abhängigkeit von $a$ und $b$ für die Beobachtungen $C$ auf die Differenz

$$
w_{11} w_{22}-w_{12} w_{21}=\delta \text {. . . . }
$$

und für die Beobachtungen $\mathrm{C}_{1}$ auf die Differenz

zu gründen.

$$
w_{11}-w_{21}=w_{22}-w_{12}=\delta^{\prime} \cdot \text {. . . }
$$

Die $z$ und $w$ sind induktiv bestimmte Häufigkeiten, die ganze Mannigfaltigkeiten von Werten repräsentieren. Dies gilt darum auch von $\delta$ und $\delta^{\prime}$. Das arithmetische Mittel aller durch $\delta$ repräsentierten Werte ist gleich $\left(1-\frac{1}{m}\right) \delta$; die mittlere quadratische Abweichung $q$ wird durch die Gleichung ${ }^{1}$ )

1) Die Herleitung der angegebenen Bestimmungen findet man in meiner Abhandlung nie Bestimmung der Abhängigkeit zwischen den Merkmalen eines Gegenstandes", S. $12 \mathrm{f}$. 


$$
\left.\begin{array}{rl}
m^{3} q^{2}= & -2(m-1)(2 m-3) \delta^{2}+(m-1)(m-2) A \\
& +(m-1) B \\
A= & w_{11} w_{22}\left(w_{11}+w_{22}\right)+w_{12} w_{21}\left(w_{12}+w_{21}\right) \\
B= & w_{11} w_{22}+w_{12} w_{21}
\end{array}\right\}
$$

bestimmt. Die Gesamtheit der Werte wird somit durch

$$
\left(1-\frac{1}{m}\right) \delta \pm q \quad \cdot \quad \cdot \quad \text {. . . }
$$

angedeutet. - Die zu $\delta^{\prime}$ gehörende Mannigfaltigkeit wird durch

$$
\left.\begin{array}{c}
\delta^{\prime} \pm q^{\prime} \\
m q^{\prime 2}=w_{11}\left(1-w_{11}\right)+w_{21}\left(1-w_{21}\right)
\end{array}\right\} .
$$

repräsentiert.

Eine vollkommene Abhängigkeit ist vorhanden, wenn entweder nur $a_{1}$ mit $b_{1}$ und $a_{2}$ mit $b_{2}$ oder nur $a_{1}$ mit $b_{2}$ und $a_{2}$ mit $b_{1}$ vereint vorkommt. Es ist alsdann entweder $w_{12}=w_{21}=0$ oder $w_{11}=w_{22}=0$, so daß für Schema C

$$
\delta=w_{11} w_{22} \text { oder } \delta=-w_{12} w_{21} \text {. . . }
$$

mit der aus (74) folgenden Bedingung

$$
w_{11}+w_{22}=1 \text { oder } w_{12}+w_{21}=1
$$

und für Schema $C_{1}$ in Übereinstimmung mit (73)

sich ergibt.

$$
\delta^{\prime}=+1 \text { oder } \quad \delta^{\prime}=-1 \quad . \quad . \quad .
$$

Demgemäß erreicht $\delta^{\prime}$ bei vollkommener Abhängigkeit den maximalen Betrag +1 oder -1 , je nachdem das Eintreten von $a$ und das Eintreten von $b$ sich wechselweise und ausnahmslos bedingen oder ausschließen, so $d a ß \delta^{\prime}$ als das zwischen +1 und - 1 schwankende Maß der Abhängigkeit in Anspruch genommen werden kann.

Der Wert $\delta$ kann hingegen bei vollkommener Abhängigkeit jeden positiven oder negativen Betrag $+w(1-w)$ oder - $w(1-w)$, wo $w<1$, annehmen und erreicht den größtmöglichen Betrag $+1 / 4$ oder $-1 / 4$ erst dann, wenn $w_{11}=w_{22}$ $=1 / 2$ oder $w_{12}=w_{21}=1 / 2$. Um die hierdurch bedingte Unsicherheit des Abhängigkeitsmaßes zu vermeiden, empfiehlt es sich, neben $w_{11} w_{22}-w_{12} w_{21}=\delta$ auch noch den Wert

$$
w_{11} w_{22}+w_{12} w_{21}=\sigma \text {. . . . . }
$$


zu bestimmen, dem die Mannigfaltigkeit

$$
\left.\begin{array}{c}
\left(1-\frac{1}{m}\right) \sigma \pm q_{1} \\
m^{3} q_{1}^{2}=-2(m-1)(2 m-3) \sigma^{2}+(m-1)(m-2) A \\
+(n i-1) B
\end{array}\right\}
$$

[wo $A$ und $B$ aus (79) zu entnehmen ist] zugehört. Alsdann erhält man in dem Quotienten

$$
\frac{\delta}{\sigma}=\frac{w_{11} w_{22}-w_{12} w_{21}}{w_{11} w_{22}+w_{12} w_{21}}=\frac{z_{11} z_{22}-z_{12} z_{21}}{z_{11} z_{22}+z_{12} z_{21}} \text {. . }
$$

ein zwischen +1 und -1 schwankendes $\mathrm{Mab}$ der Abhängigkeit. Der Quotient $\delta: \sigma$ repräsentiert eine Mannigfaltigkeit von Quotienten, die sich den Mannigfaltigkeiten der Werte $\delta$ und $\sigma$ zuordnet. Es kann indessen für ausreichend angesehen werden, wenn man die Mannigfaltigkeit von Quotienten unter Vernachlässigung von Größen von der Ordnung 1: $m$ durch den Quotienten

darstellt.

$$
\left.\begin{array}{rl}
\frac{\delta \pm q}{\sigma \pm q_{1}} & =\frac{\delta}{\sigma}\left\{1 \pm \frac{q}{\delta} \pm \frac{q_{1}}{\sigma}\right\} \\
m q^{2} & =-4 \delta^{2}+A \\
m q_{1}^{2} & =-4 \sigma^{2}+A
\end{array}\right\} .
$$

Der zwischen +1 und -1 schwankende Grad der Abhängigkeit für einen objektiven Zustand oder Vorgang $a$ einerseits und einen Bewußtseinsinhalt $b$ andererseits wird somit für die dem Schema C sich einfügenden Beobachtungen durch den Quotienten $\delta: \sigma$ (86) und für die nach Schema $C_{1}$ angestellten Beobachtungen durch die Differenz $\delta^{\prime}(78)$ bestimmt. Hierbei sind $\delta, \delta^{\prime}$, $\sigma$ Repräsentanten der durch (80), (81), (85) angedeuteten Wertsysteme, während das System der Quotienten, die durch $\delta: \sigma$ vertreten werden, mittels (87) charakterisiert wird.

Ebenso wie für einen einzelnen objektiven Zustand oder Vorgang $a$ und einen einzelnen Bewußtseinsinhalt $b$ kann man auch für eine Reihe von Objekten $a_{1}, a_{2}, a_{3} \ldots$ und eine Reihe von Bewußtseinsinhalten $b_{1}, b_{2}, b_{3} \ldots$ den Grad der Abhängigkeit bestimmen. 
Es ist möglich, daß man beim Beobachten der $a$ und der $b$ nicht die jeweils auftretenden Glieder selbst feststellen, sondern nur je zwei beobachtete Fälle vergleichen und angeben kann, ob die $a$ und $b$ im nämlichen oder im entgegengesetzten Sinne sich ändern. Hat man in diesem Falle die aus $n$ Doppelgliedern

$$
\left(a_{\alpha} b_{\beta}\right),\left(a_{\gamma} b_{\delta}\right),\left(a_{\varepsilon} b_{\eta}\right) \ldots
$$

bestehende Reihe erhalten, so kann man jedes Doppelglied mit jedem in der Reihe folgenden vergleichen und, so oft der Übergang der $a$ und der $b$ in gleichem Sinne erfolgt, ein + , anderenfalls ein - notieren. Die so sich ergebenden $1 / 2 n(n-1)$ Paare von Doppelgliedern liefern demgemäß $i$ Pluszeichen und $k$ Minuszeichen, wo

$$
i+k=1 / 2 n(n-1) \text {. . . . . }
$$

Ist nun keine Abhängigkeit zwischen der Reihe der $a$ und der Reihe der $b$ vorhanden, so hat man bei unbegrenzter Wiederholung der $n$ Beobachtungen im Durchschnitt aller Fälle

$$
1 / 4 n(n-1) \text {. }
$$

gleichsinnige und ebensoviele entgegengesetzt gerichtete Successionen der $a$ und der $b$ zu erwarten, wobei die mittlere quadratische Abweichung der in den einzelnen Fällen sich ergebenden Anzahlen von Successionen der einen und der anderen Art durch

$$
1 / 12 \sqrt{2 n(n-1)(2 n+5)} \text {. . . . . }
$$

angegeben wird ${ }^{1}$ ).

Aus der Abweichung von diesen Werten kann man die Abhängigkeit zwischen den $a$ und den $b$ ersehen. Tritt beispielsweise der Reihenfolge $1,2,3,4,5$ in der $a$-Reihe die durch $1,3,5,4,2 \mathrm{zu}$ bezeichnende Folge in der $b$-Reihe zur Seite, so ergeben sich aus

$$
(1,1),(2,3),(3,5),(4,4),(5,2)
$$

$i=6$ Pluszeichen und $k=4$ Minuszeichen. Beim Fehlen jeglicher Abhängigkeit hat man hingegen $i=5$ Pluszeichen und $k=5$ Minuszeichen mit der mittleren quadratischen Abweichung

1) Vgl. „Die Bestimmung der Abhängigkeit zwischen den Merkmalen eines Gegenstandes", S. 32. 
2,04 zu erwarten, so daß die beobachtete Abweichung kleiner ist als diejenige, die bei voller Unabhängigkeit durchschnittlich auftritt.

Kann man aber die jeweils zur Beobachtung gelangenden Glieder $a_{\alpha}, b_{\beta} ; a_{\gamma}, b_{\delta} ; a_{\varepsilon}, b_{\eta} \ldots$ bestimmt angeben, so lassen sich die relativen Häufigkeiten für das vereinigte Auftreten der einzelnen $a$ und $b$ feststellen.

Sind die $a$ oder die $b$ nach Willkür herstellbar, so können die Beobachtungen für jedes Glied der einen oder der anderen Reihe unabhängig von den übrigen Gliedern ausgeführt werden. Setzt man beispielsweise die Glieder $b_{1}, b_{1}, b_{3} \ldots$ als willkürlich herstellbar voraus und bezeichnet man für ein beliebiges $b_{\lambda}(\lambda=1,2,3 \ldots)$ die Häufigkeiten der Glieder $a_{1}, a_{2}, a_{3} \ldots$ durch $z_{\lambda 1}, z_{\lambda 2}, z_{\lambda 3} \ldots$, so kaun man die Beobachtungen in ein Schema von folgender Form eintragen:

\begin{tabular}{|c|c|c|c|c|c|c|}
\hline \multicolumn{2}{|c|}{$b_{1}$} & \multicolumn{2}{|c|}{$b_{2}$} & \multicolumn{2}{|c|}{$b_{3}$} & $\cdots$ \\
\hline$a$ & $z(1)$ & $a$ & $z(2)$ & $a$ & $z(3)$ & . . \\
\hline$a_{1}$ & $z_{11}$ & $a_{1}$ & $z_{21}$ & $a_{1}$ & $z_{31}$ & . . \\
\hline$a_{2}$ & $z_{12}$ & $a_{2}$ & $z_{22}$ & $a_{2}$ & $z_{32}$ & . . . \\
\hline$a_{3}$ & $z_{13}$ & $a_{3}$ & $z_{23}$ & $a_{3}$ & $z_{33}$ & . . \\
\hline$a_{4}$ & $z_{14}$ & $a_{4}$ & $z_{24}$ & $a_{4}$ & $z_{34}$ & . . \\
\hline . & . & . & . & . & $\cdot$ & \\
\hline . & . & . & . & . & . & \\
\hline. & . & . & . & . & . & \\
\hline
\end{tabular}

Läßt sich jedoch die Beobachtung der einzelnen $b_{1}, b_{2}, b_{3} \ldots$ oder $a_{1}, a_{2}, a_{3} \ldots$ nicht unabhängig voneinander ausführen, so erbält man an Stelle der einzelnen Reibe ein System von Beobachtungen, das sich folgendem Schema einfügt:

\begin{tabular}{c||c|c|c} 
& $b_{1}$ & $b_{2}$ & $b_{3} \ldots$ \\
\hline \hline$a_{1}$ & $z_{11}$ & $z_{21}$ & $z_{31} \ldots$ \\
$a_{2}$ & $z_{12}$ & $z_{22}$ & $z_{32} \ldots \cdot$ \\
$a_{3}$ & $z_{13}$ & $z_{23}$ & $z_{33} \cdots$ \\
$a_{4}$ & $z_{14}$ & $z_{24}$ & $z_{34} \cdots$ \\
$\cdot$ & $\cdot$ & $\cdot$ & $\cdot$ \\
$\cdot$ & $\cdot$ & $\cdot$ & $\cdot$ \\
. & $\cdot$ & $\cdot$ & $\cdot$
\end{tabular}


Diese Schemata reduzieren sich auf $C_{1}$ und $C$, wenn in der $a$-Reihe und $b$-Reihe nur je zwei Glieder $a_{1}, a_{2}$ und $b_{1}, b_{2}$ auftreten.

Um nun aus den Reihen $\mathrm{D}_{1}$ oder aus dem Systeme D den Grad-der Abhängigkeit für die $a$ einerseits und für die $b$ andererseits $\mathrm{zu}$ bestimmen, ist es am zweckdienlichsten, statt die $z$-Werte unmittelbar zu betrachten, Mittelwerte der $z$ zu benutzen.

Man hat demgemäß für jede einzelne, im Schema $D_{1}$ auftretende Beobachtungsreihe die früher $(\S 15)$ entwickelte $\mathrm{Me-}$ thode der Mittelwerte in Anwendung zu bringen. Stimmen alsdann die Mittelwerte für die verschiedenen Reihen völlig oder nahezu überein, so besteht keine Abhängigkeit der $a$ von den $b$. Ändern sich aber die Mittelwerte beim Übergange von einem Gliede der $b$-Reihe zu einem anderen, so gibt sich hierin die Abhängigkeit zu erkennen. Die Größe der Änderung kann als Maß der Abbängigkeit in Anspruch genommen werden.

Es sind ferner für das Beobachtungssystem D entsprechende Mittelwerte zu benutzen, die man bei einer Gesamtzahl $m$ der Beobachtungen für die beliebig gewählten Ausgangswerte $a_{0}$ und $b_{0}$ aus

$$
\begin{aligned}
& \left.\varepsilon(\varrho, \sigma)=z_{11}\left(a_{1}-a_{0}\right)^{\circ}\left(b_{1}-b_{0}\right)^{\sigma}+z_{12}\left(a_{1}-a_{0}\right)^{\circ}\left(b_{2}-b_{0}\right)^{\sigma}+\cdots\right) \\
& \left.\begin{array}{l}
+z_{21}\left(a_{2}-a_{0}\right)^{\circ}\left(b_{1}-b_{0}\right)^{\sigma}+z_{22}\left(a_{2}-a_{0}\right)^{\circ}\left(b_{2}-b_{0}\right)^{\sigma}+\cdots \\
+. \cdot \text {. . . . . . . . . . . . . . . }
\end{array}\right\}
\end{aligned}
$$

erhält, wenn

$$
\varepsilon_{\rho \sigma \sigma}^{\rho+\sigma}=\varepsilon(\varrho, \sigma) \text { und } \quad \varepsilon_{\varphi \sigma \sigma}=\sqrt[\varrho+\sigma]{\varepsilon(\varrho, \sigma)} \ldots
$$

gesetzt wird, wofern man darauf achtet, bei geradzahligem $\rho+\sigma$ den absoluten Betrag von $\varepsilon(\varrho \sigma)$ zugrunde zu legen, wenn dieser Wert negativ ist. Wie auf Grund dieser Mittelwerte $\varepsilon_{\rho \sigma}$ und Mittelwertpotenzen $\varepsilon(\varrho \sigma)$ die Abhängigkeitsbestimmung auszuführen ist, habe ich in der Abhandlung "Die Bestimmung der Abhängigkeit zwischen den Merkmalen eines Gegenstandes" angegeben. Weitere Ausführungen über die „aus Potenzsummen reeller Größenpaare gebildeten Mittelwerte" gibt meine "Theorie der Kollektivgegenstände". 


\section{Der Typus der Beobachtungsreihe.}

Da eine aus $\nu$ Gliedern bestehende Beobachtungsreihe nach $\S 15$ (S. 98) durch die Mittelwerte $\varepsilon_{1}, \varepsilon_{2}, \varepsilon_{3} \ldots \varepsilon_{v-1}$ vollständig und durch eine kleinere Anzahl von Mittelwerten näherungsweise bestimmt wird, so muß der Typus der Beobachtungsreihe in diesen Mittelwerten hervortreten. Es fragt sich nur, in welchem Maße dies bei den einzelnen Mittelwerten und bei gewissen aus ihnen abgeleiteten Werten der Fall ist.

In dieser Hinsicht ist zunächst zu bemerken, daß durch den Mittelwert erster Ordnung $\varepsilon_{1}$ das arithmetische Mittel als Repräsentant der ganzen Beobachtungsreihe bestimmt wird. Darum ist es durchgängig als Ausgangswert der Abweichungen zur Berechnung der Mittelwerte zu benutzen. Für diesen Ausgangswert nimmt $\varepsilon_{1}$ den Wert Null an.

Wird nun das arithmetische Mittel als Ausgangswert gewählt, so dient der Mittelwert zweiter Ordnung $\varepsilon_{2}$ als Maß für die Streuung der Werte innerhalb der Beobachtungsreihe, da $\varepsilon_{2}$ um so größer wird, je größer das Gebiet ist, auf das sich die beobachteten Werte verteilen.

Der Mittelwert dritter Ordnung $\varepsilon_{3}$ ist für eine symmetrische Beobachtungsreihe, in welcher positive und gleich große negative Abweichungen vom arithmetischen Mittel mit der nämlichen Häufigkeit auftreten, gleich Null. Dasselbe gilt von allen anderen Mittelwerten ungerader Ordnung. Darum hat $\varepsilon_{3}$ im Verein mit den sonst noch etwa berechneten Mittelwerten ungerader Ordnung als Maß der Asymmetrie zu gelten. Dabei ist der absolute Betrag dieser Werte nicht mit besonderem Gewichte in Anschlag zu bringen. Denn er ändert sich rasch bei einer Veränderung des Ausgangswertes, wie dies Fig. 5 vor Augen stellt. Dort ist der Verlauf der Mittelwerte $\eta_{1}, \eta_{2}, \eta_{3}, \eta_{4}, \eta_{5}$ auf Grund der Gleichungen

$$
\left.\begin{array}{l}
\eta(1)=-x, \eta(2)=5,04-x^{2}, \eta(3)=14,0-15,1 x-x^{3} \\
\eta(4)=114-56,0 x+30,2 x^{2}+x^{4} \\
\eta(5)=670-570 x+140 x^{2}-50,4 x^{3}-x^{5}
\end{array}\right\}
$$

(wo $\eta(\varrho)=\eta_{i}^{0}$ für $\varrho=1,2,3 \ldots$ gesetzt ist) für den als Abszisse in der Koordinatenachse $B B_{1}$ abgetragenen Ausgangs- 
wert $x$ gezeichnet. Die Ordinatenachse $A A_{1}$ enthält für $x=0$ die dem arithmetischen Mittel als Ausgangswert zugehörigen Mittelwerte. Die Mittelwerte wurden aus der Wertenreihe ${ }^{1}$ )

$$
\begin{array}{cccccccccccc}
a=4 & 5 & 6 & 7 & 8 & 9 & 10 & 11 & 12 & 13 & 14 & 15 \\
\hline z=1 & 12 & 13 & 16 & 7 & 4 & 4 & 4 & 1 & 0 & 1 & 1
\end{array}
$$

berechnet, die den „Experimentellen Beiträgen zur Untersuchung des Gedächtnisses" von G. E. Müller und. F. Schumann entnommen ist.

\section{Fig. 5.}

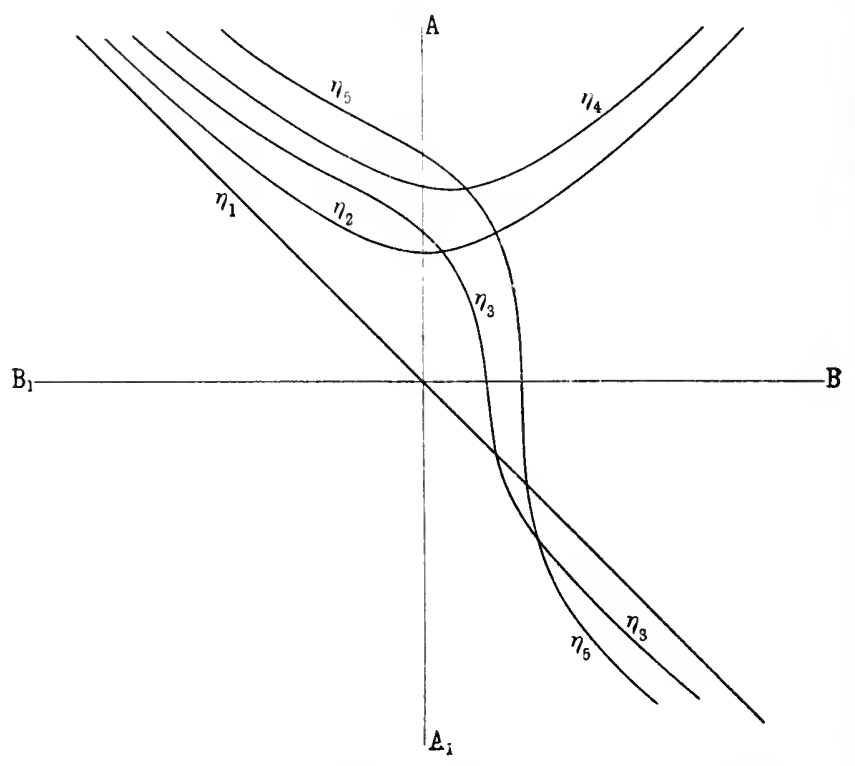

Im Gegensatz zu den Mittelwerten ungerader Ordnung zeigen diejenigen von gerader Ordnung einen ruhigen Verlauf. Darum ist $\varepsilon(4)$ oder $\varepsilon_{4}$ neben dem Mittelwerte zweiter Ordnung, der als Streuungsmaß dient, zur Kennzeichnung des Beobachtungstypus zu verwerten. Da man geneigt sein wird, das Gausssche Fehler-

1) Die Werte $a$ geben an, wie oft eine gegebene Silbenreihe durchlesen werden mußte, um sie auswendig hersagen zu können. Die Anzahlen $z$ bezeichnen die Häufigkeiten der beobachteten Werte $a$. Vgl. "Theorie der Kollektivgegenstände", S. 127, 194. 
gesetz, für welches [nach (63), S. 99] $\varepsilon(4)=3 . \varepsilon(2)^{2}$, zur Orientierung zu benutzen, so liegt es nahe, an Stelle von $\varepsilon(4)$ oder $\varepsilon_{4}=\sqrt[4]{\varepsilon(4)}$ die Differenz $\varepsilon(4)-3 . \varepsilon(2)^{2}$ oder ihre vierte Wurzel als charakteristischen Wert einzuführen.

Durch den Fortgang zu Mittelwerten von noch böberer Ordnung könnte man eine noch schärfere Charakterisierung der Beobachtungsreihe zu erreichen suchen. Es dürfte sich indessen die Benutzung der Mittelwerte erster bis vierter Ordnung im allgemeinen als ausreichend erweisen.

Folgendes Beispiel möge zur Erläuterung dienen:

Fechner hat vier Striche von der Länge $l=16 \mathrm{~mm}, 24 \mathrm{~mm}$, $36 \mathrm{~mm}, 53,7 \mathrm{~mm}$ verschiedenen Personen, im ganzen 49 Männern und 35 Frauen, vorgelegt mit der Aufforderung, in der am wohlgefälligsten erscheinenden Entfernung über jedem Strich einen Punkt (in ähnlicher Weise wie über dem I-Strich den I-Punkt) anzubringen. Die Entfernungen der Punkte von den oberen Enden der Striche wurden gemessen und in eine Liste eingetragen, die sich im wissenschaftlichen Nachlasse Fechners, der im Fechner-Archiv ${ }^{1}$ ) der Sächsischen Gesellschaft der Wissenschaften aufbewahrt wird, vorgefunden hat.

Dieser Liste habe ich die vier Doppelreihen der Tabelle 6 entnommen, wo für jedes $b$ in der Kolumne unter $a$ die Entfernungen der Punkte von den Strichen in Millimetern, unter $z(1)$ die den 49 Männern, unter $z(2)$ die den 35 Frauen zugehörenden Häufigkeiten der einzelnen Entfernungen verzeichnet sind.

Die aus den acht Reihen berechneten Mittelwerte sind in der Tabelle 7 verzeichnet. Da die Anzahlen der auf jede Reihe fallenden Beobachtungen klein und dementsprechend die mittleren quadratischen Abweichungen der Mittelwerte groß sind, so lohnte es sich nicht, bei der Berechnung der Mittelwerte nach dem im Anhange angegebenen Verfahren von den Mitten der Intervalle, in welche die arithmetischen Mittelwerte fallen, zu den Mittelwerten selbst noch überzugehen. Darum findet man neben dem arithmetischen Mittel $b$ die für die zugehörige Intervallmitte als Ausgangswert berechneten Mittelwertpotenzen. $\eta(2), \eta(3), \eta(4)$

1) Einen Bericht über den Inhalt des Fechner-Archivs habe ich in den Berichten der Sächsischen Gesellschaft der Wissenschaften (Math.-phys. Klasse, 1905) gegeben. 
Tabelle 6 .

\begin{tabular}{|c|c|c|c|c|c|c|c|c|c|c|c|}
\hline \multicolumn{3}{|c|}{$l=16 \mathrm{~mm}$} & \multicolumn{3}{|c|}{$l=24 \mathrm{~mm}$} & \multicolumn{3}{|c|}{$l=36 \mathrm{~mm}$} & \multicolumn{3}{|c|}{$l=53,7 \mathrm{~mm}$} \\
\hline$a$ & $z(1)$ & $z(2)$ & $a$ & $z(1)$ & $z(2)$ & $a$ & $z(1)$ & $z(2)$ & $a$ & $z(1)$ & $z(2)$ \\
\hline 2 & 1,5 & - & 3 & 0,5 & - & 4 & 1 & - & 5 & 0,5 & 0,5 \\
\hline 3 & 1,5 & - & 4 & 1,5 & 2 & 5 & 1 & - & 6 & 0,5 & 1,5 \\
\hline 4 & 4,5 & 2,5 & 5 & 3,5 & 2 & 6 & 2 & 2 & 7 & 0 & 1,5 \\
\hline 5 & 7 & 5,5 & 6 & 3 & 0 & 7 & 1 & 2 & 8 & 0 & 0,5 \\
\hline 6 & 9,5 & 4 & 7 & 6 & 3 & 8 & 2 & 2,5 & 9 & 2 & 1 \\
\hline 7 & 7 & 6,5 & 8 & 2 & 3 & 9 & 2 & 2 & 10 & 2 & 1 \\
\hline 8 & 9 & 3 & 9 & 6 & 6,5 & 10 & 5 & 1,5 & 11 & 2,5 & 0 \\
\hline 9 & 4 & 2 & 10 & 2 & 5,5 & 11 & 4 & 3 & 12 & 2,5 & 0 \\
\hline 10 & 4,5 & 3,5 & 11 & 9,5 & 1,5 & 12 & 3,5 & 1 & 13 & 1 & 2,5 \\
\hline 11 & 0,5 & 3 & 12 & 8 & 5 & 13 & 1,5 & 1,5 & 14 & 1 & 1,5 \\
\hline 12 & - & 2,5 & 13 & 2 & 1,5 & 14 & 3 & 2 & 15 & 1 & 1 \\
\hline 13 & - & 1,5 & 14 & 2,5 & 0 & 15 & 4,5 & 3,5 & 16 & 3 & 0 \\
\hline 14 & - & 0 & 15 & 1,5 & 0,5 & 16 & 6,5 & 3,5 & 17 & 1,5 & 1,5 \\
\hline 15 & - & 0,5 & 16 & 1 & 2,5 & 17 & 5,5 & 2,5 & 18 & 3 & 2 \\
\hline 16 & - & 0,5 & 17 & - & 1 & 18 & 0,5 & 0,5 & 19 & 1,5 & 2 \\
\hline & & & 18 & - & 0 & 19 & 0 & 3 & 20 & 2 & 1,5 \\
\hline & & & 19 & - & 0 & 20 & 2 & 0,5 & 21 & 0 & 0 \\
\hline & & & 20 & - & 0 & 21 & 1 & 0 & 22 & 1,5 & 1,5 \\
\hline & & & 21 & - & 0 & 22 & 2 & 0,5 & 23 & 4,5 & 2,5 \\
\hline & & & 22 & - & 1 & 23 & 1 & 1,5 & 24 & 4 & 4 \\
\hline & & & & & & 24 & - & 0,5 & 25 & 3,5 & 3,5 \\
\hline & & & & & & 25 & - & 0,5 & 26 & 3 & 1 \\
\hline & & & & & & 26 & - & 0 & 27 & 2 & 1,5 \\
\hline & & & & & & 27 & - & 0 & 28 & 1 & 1 \\
\hline & & & & & & 28 & - & 1 & 29 & 3,5 & 0 \\
\hline & & & & & & & & & 31 & 1 & 0 \\
\hline & & & & & & & & & 34 & 1 & 0 \\
\hline & & & & & & & & & 36 & - & 1 \\
\hline & & & & & & & & & 38 & - & 1 \\
\hline
\end{tabular}

angegeben. Durch (1) sind die den Männern, durch (2) die den Frauen zugehörigen Werte markiert.

Man ersieht hieraus, daß die durchschnittliche Entfernung der Punkte von dem zugehörigen Striche mit der Länge des Striches wächst, und zwar in der Hauptsache in gleichem Ver- 
Tabelle 7.

\begin{tabular}{|c|c|c|c|c|c|c|}
\hline$l$ & $=$ & & 16 & 24 & 36 & 53,7 \\
\hline \multirow{2}{*}{\multicolumn{2}{|c|}{$b=$}} & $f(1)$ & $6,6 \pm 0,3$ & $9,7 \pm 0,4$ & $13,5 \pm 0,7$ & $20,1 \pm 1,0$ \\
\hline & & $\mid(2)$ & $8,1 \pm 0,5$ & $10,3 \pm 0,6$ & $14,2 \pm 0,9$ & $19,6 \pm 1,3$ \\
\hline \multirow{2}{*}{\multicolumn{2}{|c|}{$\eta(2)=$}} & $\int(1)$ & $4,4 \pm 0,8$ & $9,3 \pm 1,5$ & $21,0 \pm 3,0$ & $46,3 \pm 6,9$ \\
\hline & & (2) & $8,3 \pm 1,8$ & $14,2 \pm 4,4$ & $29,2 \pm 6,6$ & $60,3 \pm 13,6$ \\
\hline \multirow{2}{*}{\multicolumn{2}{|c|}{$\eta(3)=$}} & $\int(1)$ & $-6 \pm 4$ & $-13 \pm 11$ & $-30 \pm 38$ & $-50 \pm 110$ \\
\hline & & (2) & $+15 \pm 14$ & $+56 \pm 51$ & $+91 \pm 93$ & $-49 \pm 256$ \\
\hline \multirow{2}{*}{\multicolumn{2}{|c|}{$\eta(4)=$}} & $\{(1)$ & $48 \pm 17$ & $200 \pm 56$ & $1083 \pm 299$ & $4451 \pm 1243$ \\
\hline & & $\{(2)$ & $180 \pm 95$ & $887 \pm 583$ & $2391 \pm 1128$ & $10070 \pm 3115$ \\
\hline$\eta(4)-1$ & & $\int(1)$ & -8 & - 59 & -241 & -1878 \\
\hline $3 \eta(2)^{2}$ & & \{ & -25 & +279 & -175 & -848 \\
\hline
\end{tabular}

hältnis. Das Verhältnis $b: l$ ist jedoch nicht vollkommen konstant; es nimmt bei zunehmendem $l$ für die Männer von 0,41 bis 0,37, für die Frauen in stärkerem $\mathrm{Maße}$ von 0,50 bis 0,36 ständig ab. Das Verhältnis des goldenen Schnittes, das den Wert $b: l=0,6$ fordern würde, spielt hierbei keine Rolle. - Die Streuung der Punkte wächst gleichfalls mit der Länge des Striches, jedoch so, daß der relative Streuungswert $\eta_{2}: l$ für die Männer konstant gleich 0,13, für die Frauen von 0,18 über 0,16 und 0,15 bis zu 0,14 abnimmt. - Von besonderem Interesse sind die Werte $\eta(3)$, da sie ein gegensätzliches Verhalten der Männer und Frauen vor Augen stellen und den Nachweis erbringen, daß sich das spezifisch Männliche und Weibliche sogar noch im Tüpfchen auf dem I zeigt. Es ist nämlich $\eta(3)$ für die Männer durchweg negativ, für die Frauen hingegen positiv, mit Ausnahme des zu $l=53,7$ gehörenden negativen Wertes, der jedoch durch positive Werte $\eta(5), \eta(7) \ldots$ aufgewogen wird. Hierdurch zeigt sich, daß bei gleichem arithmetischem Mittel und gleicher Streuung die Mänuer geneigt sind, im extremen Falle pedantisch am Strich zu kleben, während die Frauen sorgloser oder schwungvoller verfahren und so nach der entgegengesetzten Richtung zu extrem vom Strich abweichenden Punkten kommen. - Die Differenz 
$\eta(4)-3 \cdot \eta(2)^{2}$ ist mit einer einzigen, durch den extremen Wert der betreffenden Reihe bedingten Ausnahme negativ, bleibt aber innerhalb der Grenzen der zu $\eta(4)$ gehörenden mittleren quadratischen Abweichung. Es findet somit, auch wenn man von der Asymmetrie absieht, ein zwar schwaches, aber doch sicher hervortretendes Abweichen vom Typus des gewöhnlichen Fehlergesetzes statt. Der Quotient $\eta_{4}: \eta_{2}$ ist nämlich im Durchschnitt der acht Fälle gleich 1,28, während das Gausssche Fehlergesetz den Quotienten $\varepsilon_{4}: \varepsilon_{2}=1,32$ verlangt.

Als Belege mögen die in Tabelle 8 zusammengestellten Werte dienen.

Tabelle 8 .

\begin{tabular}{|c|c|c|c|c|}
\hline$l=$ & 16 & 24 & 36 & 53,7 \\
\hline \multirow{2}{*}{$b: l=\left\{\begin{array}{l}(1) \\
(2)\end{array}\right.$} & 0,41 & 0,40 & 0,37 & 0,37 \\
\hline & 0,50 & 0,43 & 0,39 & 0,36 \\
\hline \multirow{2}{*}{$\eta_{2}: l=$} & 0,13 & 0,13 & 0,13 & 0,13 \\
\hline & 0,18 & 0,16 & 0,15 & 0,14 \\
\hline \multirow{2}{*}{$\eta_{4}: \eta_{2}=$} & 1,27 & 1,23 & 1,25 & 1,20 \\
\hline & 1,27 & 1,45 & 1,29 & 1,29 \\
\hline
\end{tabular}

\section{Die Zerlegung der Beobachtungsreihe in Komponenten und die Bestimmung der Unterschieds- schwelle.}

Es ist möglich, daß bei der wiederholten Ausführung von Beobachtungen derselben Art je nach den obwaltenden Umständen oder dem Verhalten des Beobachters verschiedene Typen in den successive sich ergebenden Beobachtungsreihen hervortreten. Dann kann aber auch schon innerhalb einer und derselben Beobachtungsreihe der Beobachtungsmodus wechseln, so daß eine Mischung verschiedener Typen entsteht. Darum ist es wünschenswert, über eine Methode zu verfügen, nach der man das Vorhandensein einer solchen Mischung nachweisen und die den verschiedenen Typen zugehörenden Komponenten der Beobachtungsreihe feststellen kann. 
$\mathrm{Zu}$ diesem $\mathrm{Zwecke}$ ist der Zusammenhang zwischen den Mittelwerten von $\mu$ Beobachtungsreihen einerseits und den Mittelwerten der aus den $\mu$ Reihen zusammengesetzten Beobachtungsreihe andererseits $\mathrm{zu}$ entwickeln.

Die $\mu$ Beobachtungsreihen mögen nach dem Schema $D_{1}$ den Werten $b_{1}, b_{2}, \ldots b_{\mu}$ zugeordnet gedacht werden, wobei jedoch der einfacheren Bezeichnungsweise wegen vorausgesetzt werden soll, daß $b_{1}, b_{2}, \ldots b_{\mu}$ zugleich die den betreffenden Reihen zukommenden arithmetischen Mittelwerte darstellen. Dann erhält man für die zu $b_{k}$ gehörende Reihe, wenn die Gesamtzahl ihrer Werte durch $m_{\%}$, und die auf $b_{\%}$ als Ausgangswert bezogene Mittelwertpotenz $\varrho$ ter Ordnung für $\varrho=2,3 \ldots$ durch $\varepsilon_{2}(\varrho)$ angegeben wird:

\begin{tabular}{c|c}
\multicolumn{2}{c}{$b_{j}$} \\
\hline$a$ & $z(\lambda)$ \\
\hline$a_{1}$ & $z_{\lambda 1}$ \\
$a_{2}$ & $z_{\lambda 2}$ \\
$a_{3}$ & $z_{\lambda 3}$ \\
$\cdot$ & $\cdot$ \\
$\cdot$ & $\cdot$
\end{tabular}

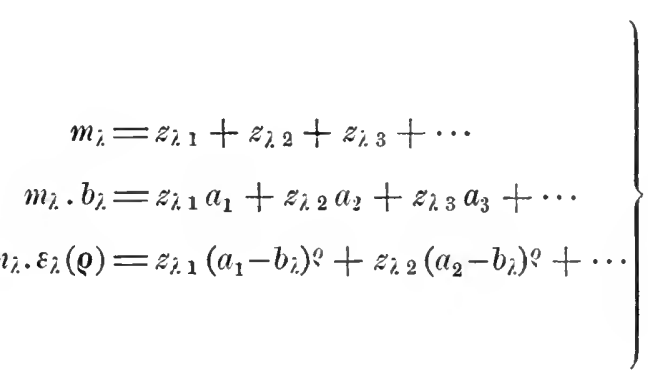

Für die durch das Zusammenlegen der $\mu$ Reihen entstehende Beobachtungsreihe sei ferner die Gesamtzahl der Werte gleich $m$, das arithmetische Mittel gleich $b$ und die auf $b$ bezogene Mittelwertpotenz $\varrho$ ter Ordnung für $\varrho=2,3 \ldots$ gleich $\varepsilon(\varrho)$, so daß man zu

\begin{tabular}{|c|c|c|}
\hline$a$ & $z$ & . \\
\hline$a_{1}$ & $z_{1}$ & $m=z_{1}+z_{2}+z_{3}+\cdots$ \\
\hline$a_{2}$ & $z_{2}$ & $m \cdot b=z_{1} a_{1}+z_{2} a_{2}+z_{3} a_{3}+\cdots$ \\
\hline$a_{3}$ & $z_{3}$ & \\
\hline$\cdot$ & · & $m \cdot \varepsilon(\varrho)=z_{1}\left(a_{1}-b\right)^{?}+z_{2}\left(a_{2}-b\right)^{?}+\cdots$ \\
\hline$\cdot$ & $\cdot$ & \\
\hline$\cdot$ & $\cdot$ & \\
\hline
\end{tabular}

gelangt. Nun ist

Lipps, Die psychischen Maßmethoden. 


$$
\left.\begin{array}{c}
-130- \\
z_{1}=z_{11}+z_{21}+\cdots+z_{\mu 1} \\
z_{2}=z_{12}+z_{22}+\cdots+z_{\mu 2} \\
z_{3}=z_{13}+z_{23}+\cdots+z_{\mu 3} \\
\text {. . . . . . . . . . }
\end{array}\right\} .
$$

Daraus folgt, wie man sich durch die unmittelbare Ausrechnung ïberzeugt:

$$
\begin{aligned}
& m=m_{1}+m_{2}+\cdots+m_{\mu} \\
& m b=m_{1} b_{1}+m_{2} b_{2}+\cdots+m_{\mu} b_{\mu} \\
& m \varepsilon(\varrho)=m_{1} \varepsilon_{1}(\varrho)+m_{2} \varepsilon_{2}(\varrho)+\cdots+m_{\mu} \varepsilon_{\mu}(\varrho) \\
& +\frac{\varrho}{1}\left(b_{1}-b\right) m_{1} \varepsilon_{1}(\varrho-1)+\cdots+\frac{\varrho}{1}\left(b_{\mu}-b\right) m_{\mu} \varepsilon_{\mu}(\varrho-1) \\
& +m_{1}\left(b_{1}-b\right)^{o}+m_{2}\left(b_{2}-b\right)^{\circ}+\cdots+m_{\mu}\left(b_{\mu}-\dot{b}\right)^{\rho}
\end{aligned}
$$

Ist insbesondere $\mu=2$, so erhält man

$$
\begin{aligned}
m & =m_{1}+m_{2} \\
m b & =m_{1} b_{1}+m_{2} b_{2} \\
m \varepsilon(2) & =m_{1} \varepsilon_{1}(2)+m_{2} \varepsilon_{2}(2) \\
& +m_{1}\left(b_{1}-b\right)^{2}+m_{2}\left(b_{2}-b\right)^{2} \\
m \varepsilon(3) & =m_{1} \varepsilon_{1}(3)+m_{2} \varepsilon_{2}(3) \\
& +3 m_{1}\left(b_{1}-b\right) \varepsilon_{1}(2)+3 m_{2}\left(b_{2}-b\right) \varepsilon_{2}(2) \\
& +m_{1}\left(b_{1}-b\right)^{3}+m_{2}\left(b_{2}-b\right)^{3} \\
m \varepsilon(4) & =m_{1} \varepsilon_{1}(4)+m_{2} \varepsilon_{2}(4) \\
& +4 m_{1}\left(b_{1}-b\right) \varepsilon_{1}(3)+4 m_{2}\left(b_{2}-b\right) \varepsilon_{2}(3) \\
& +6 m_{1}\left(b_{1}-b\right)^{2} \varepsilon_{1}(2)+6 m_{2}\left(b_{2}-b\right)^{2} \varepsilon_{2}(2) \\
& +m_{1}\left(b_{1}-b\right)^{4}+m_{2}\left(b_{2}-b\right)^{4}
\end{aligned}
$$

Auf Grund der Gleichungen (97) und (98) kann man entscheiden, ob eine gegebene, durch $m, b, \varepsilon(2), \varepsilon(3)$... charakterisierte Beobachtungsreihe als eine Mischung aus $\mu$ oder insbesondere aus zwei Beobachtungsreihen mit den arithmetischen Mittelwerten $b_{\lambda}$ und den Mittelwertpotenzen $\varepsilon_{\lambda}(2), \varepsilon_{\lambda}(3) \ldots$ anfgefaßt werden kann, indem man feststellt, ob es ein System von Werten $m_{1}, m_{2} \ldots m_{\mu}$ oder insbesondere ein Wertenpaar $m_{1}, m_{2}$ gibt, das die Gleichungen (97) oder insbesondere (98) befriedigt. 
Auf diesem Wege kann man beispielsweise entscheiden, ob es nur zwei typische Formen für die Ausführung von Reaktionen gibt, die man als muskuläre und sensorielle Reaktionsweise zu unterscheiden pflegt, oder ob nicht vielmehr die genannten Formen die extremen Fälle einer stetig abgestuften Folge oder wenigstens einer größeren Reihe von Reaktionsformen darstellen.

Bei der Ausführung einer Reaktion wird man nämlich von Haus aus ein den persönlichen Neigungen und Gewohnheiten entsprechendes "natürliches" Verhalten beobachten. Es kann aber durch Einübung in der Regel erreicht werden, daß der Reagent nach Belieben sowohl verlängerte als sensoriell bezeichnete, wie auch verkürzte sogenannte muskuläre Reaktionen auszuführen imstande ist. Hierdurch wird die Vermutung nahegelegt, daß

Fig. 6.

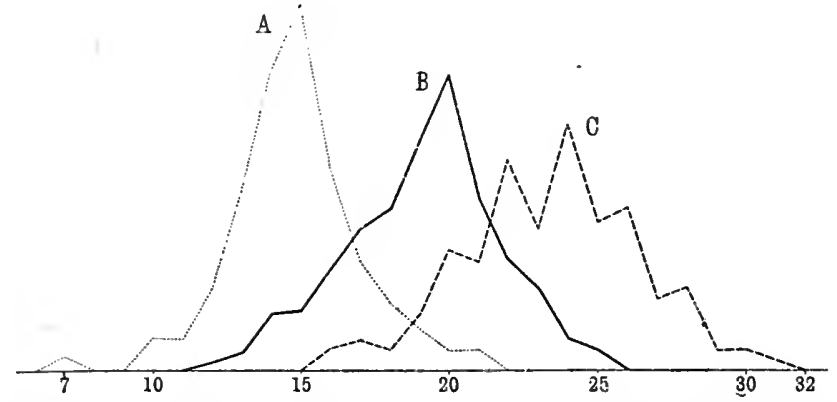

jede Reaktion entweder sensoriell oder muskulär erfolge und daß sonach die "natürliche" Reaktionsweise aus einer Mischung des sensoriellen und muskulären Typus bestebe.

Um hierüber eine Entscheidung herbeizuführen, habe ich die in der Dissertation ${ }^{1)}$ "Reaktionszeiten bei Durchgangsbeobachtungen " von Alechsieff mitgeteilten Beobachtungsreihen untersucht. Beispielshalber teile ich die von Alechsieff selbst angestellten Reaktionsversucbe in Tabelle 9 mit. Die Reaktionszeiten wurden bis auf Tausendstel Sekunden bestimmt und sodann bei der tabellarischen und graphischen Darbietung in Intervalle von je zehn Tausendstel durch Vereinigung der die nämlichen Hundertstel aufweisenden Zeiten zusammengefaßt. Die

1) Philosophische Studien (herausg. von Wundt), 16 (1900). 
Tabelle 9 .

\begin{tabular}{c|r|r|r||r|r}
\hline \multicolumn{2}{c|}{ Muskulär } & \multicolumn{2}{c||}{ natürlich } & \multicolumn{2}{c}{ sensoriell } \\
\hline$a$ & $z$ & $a$ & $z$ & $a$ & $z$ \\
\hline 7,45 & 1 & 12,45 & 1 & 16,45 & 2 \\
8,45 & 0 & 13,45 & 2 & 17,45 & 3 \\
9,45 & 0 & 14,45 & 6 & 18,45 & 2 \\
10,45 & 3 & 15,45 & 6 & 19,45 & 6 \\
11,45 & 3 & 16,45 & 10 & 20,45 & 12 \\
12,45 & 8 & 17,45 & 14 & 21,45 & 11 \\
13,45 & 18 & 18,45 & 16 & 22,45 & 21 \\
14,45 & 30 & 19,45 & 23 & 23,45 & 14 \\
15,45 & 37 & 20,45 & 30 & 24,45 & 25 \\
16,45 & 20 & 21,45 & 17 & 25,45 & 15 \\
17,45 & 11 & 22,45 & 11 & 26,45 & 16 \\
18,45 & 7 & 23,45 & 8 & 27,45 & 7 \\
19,45 & 4 & 24,45 & 3 & 28,45 & 8 \\
20,45 & 2 & 25,45 & 2 & 29,45 & 2 \\
21,45 & 2 & & -149 & 30,45 & 2 \\
& 146 & & & 31,45 & 1 \\
\hline
\end{tabular}

hierdurch bedingten Intervallmitten sind in Hundertstel Sekunden unter $a$ angegeben. Die danebenstehenden $z$-Werte bezeichnen die Häufigkeiten für das Auftreten der a. Es fallen sonach beispielsweise von den muskulären Reaktionen auf das von 130 bis 139 Tausendstel Sekunden sich erstreckende Intervall mit der Mitte 134,5 Tausendstel oder 13,45 Hundertstel, im ganzen 18 Reaktionen.

Den Verlauf der $z$-Werte veranschaulicht Fig. 6. Sie lehrt, daß in der Tat die natürlichen Reaktionen $B$ sich in der Mitte zwischen den muskulären Reaktionen $A$ und den sensoriellen Reaktionen $C$ halten, so daßj man nach dem Anblick der Kurven auf eine Mischung der natürlichen Reaktionsform an den beiden Grenzformen zu schließen versucht ist. Berechnet man aber die Mittelwerte, so findet man die in Tabelle 10 angegebenen Werte der arithmetischen Mittel $b$ und der auf $b$ bezogenen Mittelwertpotenzen $\varepsilon(2), \varepsilon(3), \varepsilon(4)$. 
Tabelle 10.

\begin{tabular}{|c|c|c|c|c|}
\hline & Reaktionsw. & muskulär & natürlich & sensoriell \\
\hline$b$ & . . . . . . . & 15,3 & 19,5 & 22,9 \\
\hline$\varepsilon(2)$ & . . . . . . . & 4,6 & 6,7 & 8,7 \\
\hline$\varepsilon(3)$ & . . . . . . . & $+0,7$ & $-4,7$ & $-2,2$ \\
\hline$\varepsilon(4)$ & . . . . . . . & 91 & 130 & 216 \\
\hline
\end{tabular}

Setzt man nun unter den $m$ natürlichen Reaktionen $m_{1}$ muskuläre und $m_{2}$ sensorielle voraus, so müßten auf Grund von (98), wenn $m_{1}: m=p$ und $m_{2}: m=q$ gesetzt wird, folgende Gleichungen bestehen:

$$
\begin{aligned}
1 & =p+q \\
19,5 & =p \cdot 22,9+q \cdot 15,3 \\
6,7 & =p \cdot 20+q \cdot 22 \\
-4,7 & =p \cdot 126-q \cdot 131 \\
130 & =p \cdot 923+q \cdot 877
\end{aligned}
$$

$\mathrm{Da}$ jedoch diese Gleichungen keine annehmbaren Lösungen zulassen, so ist es auch nicht gestattet, die natürlichen Reaktionen als eine Mischung sensorieller und muskulärer Reaktionen aufzufassen.

Durch die Zerlegung der Beobachtungsreihe in Komponenten kann man nun auch auf Grund folgender Erwägungen zu einer einfachen Bestimmung der Unterschiedsschwellenwerte gelangen.

Es wird vorausgesetzt, daß eine Unterschiedsschwelle existiert und die Schärfe der Unterscheidbarkeit zum Ausdruck bringt. Gehört nun zu einer Reihe von Beobachtungen das von den Unterschiedsschwellen $b+i$ und $b-i$ begrenzte Gebiet $b \pm i$, wo $b$ das arithmetische Mittel der beobachteten Werte angibt, so würde eine verdoppelte Unterscheidungsfähigkeit das Zerfallen des Gebietes $b \pm i$ in die von den Unterschiedsschwellen $b+i$ und $b$ einerseits, $b$ und $b-i$ andererseits begrenzten Teilgebiete und hiermit ein Zerfallen der Beobachtungsreihe in zwei, den beiden Teilintervallen zugehörende Komponenten zur Folge haben. 
In entsprechender Weise würde die Annahme einer $\mu$-fach verstärkten Unterscheidungsfähigkeit zur Zerlegung des Gebietes $b \pm i$ in $\mu$ Teile und zur Zerlegung der Beobachtungsreihe in $\mu$, den einzelnen Teilen zugehörende Komponenten führen. Schließlich kann. bei unbegrenzt verfeinerter Unterscheidung die Anzahl $\mu$ der Komponenten unbegrenzt groß angenommen werden. Es darf ferner im Interesse der Einfachheit eine übereinstimmende Beschaffenheit der Komponenten vorausgesetzt werden, so daß jede Komponente durch die nämlichen auf das jeweilige arithmetische Mittel bezogenen Mittelwertpotenzen bestimmt wird.

Werden demgemäß die arithmetischen Mittelwerte der Komponenten der Reihe nach in der Form

$$
\left.\begin{array}{c}
b_{1}=b-(\mu-1) \delta ; b_{2}=b-(\mu-3) \delta ; \cdots \\
b_{\mu}=b+(\mu-1) \delta
\end{array}\right\}
$$

vorausgesetzt (wo $\mu \delta=i$ ); werden ferner die allen Komponenten gemeinsamen Werte der, auf das jeweilige arithmetische Mittel bezogenen Mittelwertpotenzen durch $\eta(1)=0, \eta(2), \eta(3), \ldots$ angegeben und die Anzahlen der jeder Komponente zukommenden Beobachtungen $m_{1}, m_{2}, m_{3} \ldots$ übereinstimmend gleich $m: \mu$ angenommen, so erhält man aus (97), wenn noch für hinreichend große Werte $\mu$

$$
\delta^{2 x}\left\{(\mu-1)^{2 x}+(\mu-3)^{2 x}+\cdots\right\}=\frac{\delta^{2 x} \mu^{2 x+1}}{2 x+1}=\frac{i^{2 x} \mu}{2 x+1}
$$

gesetzt wird, die Beziehung:

$$
\left.\begin{array}{c}
\varepsilon(\varrho)=\eta(\varrho)+\frac{\varrho(\varrho-1)}{1.2} \eta(\varrho-2) \cdot \frac{i^{2}}{3} \\
+\frac{\varrho(\varrho-1)(\varrho-2)(\varrho-3)}{1.2 .3 .4} \eta(\varrho-4) \cdot \frac{i^{4}}{5}+\cdots
\end{array}\right\}
$$

Zur Bestimmung des Unterschiedsschwellenwertes $i$ erhält man hieraus zunächst

$$
\varepsilon(2)=\eta(2)+\frac{i^{2}}{3} \cdot \ldots . . .
$$

und weiterhin, wenn der Wert von $\varepsilon(4)$ zur Charakterisierung der Beobachtungsreihe verwendbar ist,

$$
\varepsilon(4)=\eta(4)+2 \eta(2) i^{2}+\frac{i^{4}}{5} \quad . \quad \text {. }
$$


Aus (102) folgt, da $\eta_{\text {(2) }}$ unbekannt, aber wesentlich positiv ist, die Bedingung

$$
i^{2}<3 \varepsilon(2), \quad . \quad . \quad . \quad . \quad . \quad .
$$

oder, wenn $\varepsilon_{2}^{2}=\varepsilon(2)$ gesetzt wird,

$$
i<\varepsilon_{2} \sqrt{3} \cdot \cdot \cdot \cdot \cdot \cdot(104 a)
$$

Durch die Vereinigung von (102) und (103) erhält man außerdem die Gleichung

$$
\varepsilon(4)-3 \varepsilon(2)^{2}=\eta(4)-3 \eta(2)^{2}-\frac{2 i^{4}}{15} .
$$

Hat man nun zusammengehörige Beobachtungsreihen, deren Mittelwertpotenzen zweiter und vierter Ordnung durch $\varepsilon_{1}(2)$, $\varepsilon_{1}(4) ; \varepsilon_{2}(2), \varepsilon_{2}(4) ; \varepsilon_{3}(2), \varepsilon_{3}(4) ; \ldots$ bezeichnet werden mögen, unter gleichartigen Umständen hergestellt, so daß die Werte $\eta(2)$ und $\eta(4)$ der vorauszusetzenden Komponenten für alle Reihen übereinstimmen, wäbrend die Unterschiedsschwellenwerte $i_{1}, i_{2}$, $i_{3} \ldots$ von einer Reibe zur andern sich ändern, so kann man auf Grund von (102) und (105) die Gleichungen

$$
\left.\begin{array}{rl}
\varepsilon_{1}(2) & =\eta(2)+\frac{i_{1}^{2}}{3} ; \\
\varepsilon_{1}(4)-3 \varepsilon_{1}(2)^{2} & =\eta(4)-3 \eta(2)^{2}-\frac{2 i_{1}^{4}}{15} \\
\varepsilon_{2}(2) & =\eta(2)+\frac{i_{2}^{2}}{3} ; \\
\varepsilon_{2}(4)-3 \varepsilon_{2}(2)^{2} & =\eta(4)-3 \eta(2)^{2}-\frac{2 i_{2}^{4}}{15} \\
\varepsilon_{3}(2) & =\eta(2)+\frac{i_{3}^{2}}{3} ; \\
\varepsilon_{3}(4)-3 \varepsilon_{3}(2)^{2} & =\eta(4)-3 \eta(2)^{2}-\frac{2 i_{3}^{4}}{15}
\end{array}\right\}
$$

ansetzen, aus denen $i_{1}, i_{2}, i_{3}, \ldots$ zugleich mit $\eta(2)$ und $\eta(4)$ durch Ausgleichung zu bestimmen sind. Es setzt dies jedoch voraus, daß der Wert $\varepsilon(4)-3 \varepsilon(2)^{2}$ beim Übergang von einer Beobachtungsreihe zur anderen im entgegengesetzten Sinne wie der Betrag der Unterschiedsschwelle sich ändert. Trifft dies nicht zu oder wird die Änderung durch unregelmäßige Schwankungen der beobachteten 
Werte überwuchert, so muß man darauf verzichten, die Werte $\varepsilon(4)$ auf Grund der Gleichung (105) zur Bestimmung der Unterschiedsschwelle heranzuziehen, und sich mit den in jedem Falle zutreffenden Ungleichungen (104) begnügen.

Tabelle 11.

\begin{tabular}{|c|c|c|c|c|c|}
\hline \multicolumn{2}{|c|}{$180^{\circ}$ WeiB } & \multicolumn{2}{|c|}{$140^{\circ}$ Weiß } & \multicolumn{2}{|c|}{$100^{\circ}$ Weiß } \\
\hline$a$ & $z$ & $a$ & $z$ & $a$ & $z$ \\
\hline 163,5 & 1 & 128 & 2 & 89,5 & 1,5 \\
\hline 165 & 0 & 129,5 & 4 & 91 & 3,5 \\
\hline 166,5 & 0 & 131 & 5 & 92,5 & 4,5 \\
\hline 168 & 3 & 132,5 & 10. & 94 & 11 \\
\hline 169,5 & 7 & 134 & 11,5 & 95,5 & 17 \\
\hline 171 & 8 & 135,5 & 16 & 97 & 19,5 \\
\hline 172,5 & 5 & 137 & 13,5 & 98,5 & 7,5 \\
\hline 174 & 7,5 & 138,5 & 16,5 & 100 & 2,5 \\
\hline 175,5 & 4 & 140 & 7,5 & 101,5 & 4,5 \\
\hline 177 & 8 & 141,5 & 6,5 & 103 & 3,5 \\
\hline 178,5 & 7,5 & 143 & 0,5 & & $m=75$ \\
\hline 180 & 8 & 144,5 & 2 & & $m=15$ \\
\hline 181,5 & 8 & 146 & 3 & & \\
\hline 183 & 7 & 147,5 & 2 & & \\
\hline 184,5 & 7 & & $m=100$ & & \\
\hline 186 & 7,5 & & & & \\
\hline 187,5 & 4 & & & & \\
\hline 189 & $\begin{array}{l}4 \\
0,5\end{array}$ & & & & \\
\hline $\begin{array}{l}190,5 \\
192\end{array}$ & 2 & & & & \\
\hline 193,5 & 0 & & & . & \\
\hline 195 & 0 & & & i & \\
\hline 196,5 & 1 & & & & \\
\hline & $=100$ & & & & \\
\hline
\end{tabular}

Als Beispiel wähle ich die bereits bei der Erörterung der Methode der eben merklichen Unterschiede (S. 54) erwähnten Versuche über Helligkeitsvergleiche an rotierenden, aus schwarzen und weißen Sektoren bestehenden Scheiben, wo die eine Scheibe feste, die andere verstellbare Sektoren hatte und die letztere auf 
die nämliche Helligkeit wie die erstere eingestellt wurde ${ }^{1}$ ). Solche Einstellungen auf subjektive Gleichheit wurden für fünf verschiedene Scheibenpaare vollzogen, wobei die Scheibe mit festen Sektoren aus $220^{\circ}$ Weiß und $140^{\circ}$ Schwarz; $180^{\circ}$ Weiß und $180^{\circ}$ Schwarz; $140^{\circ}$ Weiß und $220^{\circ}$ Schwarz; $100^{\circ}$ Weiß und $260^{\circ}$ Schwarz; $60^{\circ}$ Weiß und $300^{\circ}$ Schwarz bestand. Unter anderen Reihen baben sich die in Tabelle 11 verzeichneten ergeben, wo unter $a$ die durch Herstellen subjektiver Gleichheit gewonnenen Grade der weißen Sektoren der Vergleichsscheibe, unter $z$ die Häufigkeiten des Auftretens der $a$ stehen, während der Zahlenwert oberhalb der einzelnen Reihen die Größe der weißen Sektoren der Hauptscheibe angibt. Aus diesen Reihen findet man die in Tabelle 12 zusammengestellten Werte der

Tabolle 12.

\begin{tabular}{|c|c|c|c|}
\hline & $180^{\circ} \mathrm{Wei} \beta$ & $140^{\circ}$ Weiß & $100^{\circ}$ Weib \\
\hline b...... & $178,9+0,7$ & $136,6 \pm 0,4$ & $96,3 \pm 0,3$ \\
\hline$\varepsilon(2) \ldots$. & $1,5^{2}(19,5 \pm 2,3)$ & $1,5^{2}(7,7 \pm 1,1)$ & $1,5^{2}(3,9 \pm 0,7)$ \\
\hline$\varepsilon(3) .$. & $1,5^{3}(8 \pm 26)$ & $1,5^{3}(8 \pm 8)$ & $1,5^{8}(2 \pm 3)$ \\
\hline$\varepsilon(4)$ & $1,5^{4}(900 \pm 250)$ & $1,5^{4}(187 \pm 48)$ & $1,5^{4}(48 \pm 13)$ \\
\hline$\varepsilon(4)-3 \varepsilon(2)^{2}$ & $-1,5^{4} \cdot 241$ & $1,5^{4} .9$ & $1,5^{4} .2$ \\
\hline
\end{tabular}

arithmetischen Mittel $b$ und der auf $b$ bezogenen Mittelwertpotenzen $\varepsilon(2), \varepsilon(3), \varepsilon(4)$, so daß mit Rücksicht auf (104), wenn die zu $180^{\circ}, 140^{\circ}, 100^{\circ}$ gehörenden Unterschiedsschwellen der Reile nach durch $i_{1}, i_{2}, i_{3}$ bezeichnet werden,

$\begin{array}{ll}i_{1}^{2}<132 & i_{1}<11 \\ i_{2}^{2}<52 & i_{2}<7 \\ i_{3}^{2}<26 & i_{3}<5\end{array}$

und mit Rücksicht auf (106)

1) Eine ausführlichere Behandlung der Versuche findet man in der Abhandlung ${ }_{n}$ Die Mabmethoden der experimentellen Psychologie“, S. 71,83 . 


$$
\begin{aligned}
1,5^{2} \cdot 19,5 & =\eta(2)+\frac{i_{1}^{2}}{3} \\
-1,5^{4} \cdot 241 & =\eta(4)-3 \eta(2)^{2}-\frac{2 i_{1}^{4}}{15} \\
1,5^{2} \cdot 7,7 & =\eta(2)+\frac{i_{2}^{2}}{3} \\
1,5^{4} \cdot 9 & =\eta(4)-3 \eta(2)^{2}-\frac{2 i_{2}^{4}}{15} \\
1,5^{2} \cdot 3,9 & =\eta(2)+\frac{i_{3}^{2}}{3} \\
1,5^{4} \cdot 2 & =\eta(4)-3 \eta(2)^{2}-\frac{2 i_{3}^{4}}{15} .
\end{aligned}
$$

Es läßt sich aber diesen Gleichungen kein geeignetes System von Werten $i_{1}, i_{2}, i_{3}$ entnehmen, da die Werte von $\varepsilon(4)-3 \varepsilon(2)^{2}$ unregelmäßiges Schwanken statt der durch die Abnahme der Unterschiedsschwellenwerte $i_{1}, i_{2}, i_{3}$ bedingten gesetzmäßigen Zunahme zeigen. Man muß sich darum mit den aufgestellten Ungleichungen zufrieden geben. 


\section{A n h a $11 \mathrm{~g}$.}

\section{Die Berechnung der Mittelwerte.}

Zur Berechnung der Mittelwerte dient am bequemsten das in der "Theorie der Kollektivgegenstände" von mir entwickelte Verfahren, das hier kurz auseinandergesetzt werden soll.

Tabelle 13.

\begin{tabular}{|c|c|c|c|c|c|c|c|}
\hline$a$ & $z$ & $y$ & $x$ & $x$ & . & . & - \\
\hline$a_{1}$ & $z_{l}$ & $y_{1}$ & $r_{1}$ & $2 c_{1}$ & . & - & - \\
\hline$a_{2}$ & $z_{2}$ & $y_{2}$ & $x_{2}$ & $w_{\mathbf{z}}$ & - & • & - \\
\hline • & • & • & • & . & & & \\
\hline . & . & - & • & - & & & \\
\hline - & - & - & . & . & & & \\
\hline$a_{u-4}$ & $z_{u}-4$ & $y_{u-4}$ & $x_{u-4}$ & $w_{u-4}$ & & & \\
\hline$a_{u-3}$ & $z_{u-3}$ & $y_{u}-3$ & $x_{\mu-3}$ & $w^{\prime}$ & & & \\
\hline$a_{u-2}$ & $z_{\mu}-2$ & $y_{u-2}$ & $x^{\prime}$ & & & & \\
\hline$a_{u-1}$ & $z_{u-1}$ & $y^{\prime}$ & & & & & \\
\hline$a_{\mu}$ & $z_{u}$ & & & & & & \\
\hline$a_{\mu+1}$ & $z_{u}+1$ & $y^{\prime \prime}$ & & & & & \\
\hline$a_{u+2}$ & $z_{u+2}$ & $y_{u}+2$ & $x^{\prime \prime}$ & & & & \\
\hline$a_{u+3}$ & $z_{\mu}+3$ & $y_{u}+3$ & $x_{u}+3$ & $w^{\prime \prime}$ & & & \\
\hline$a_{\mu}+4$ & ${ }^{z} u+4$ & $y_{u}+4$ & $x_{\mu+4}$ & $w_{u+4}$ & & & \\
\hline • & - & - & • & - & & & \\
\hline - & - & - & • & - & & & \\
\hline • & • & • & $\cdot$ & • & & & \\
\hline$a_{v-1}$ & $z_{v}-1$ & $y_{y}-1$ & $x_{1}-1$ & $u v-1$ & & • & - \\
\hline$r_{y}$ & $z_{y}$ & $y_{i}$ & $x_{1}$ & $w_{y}$ & & - & - \\
\hline
\end{tabular}


In der Reihe der um den konstanten Betrag $2 l$ fortschreitenden Werte $a_{1}, a_{2}, a_{3} \ldots a_{v}$, denen die Anzahlen $z_{1}, z_{2}, z_{3} \ldots z_{v}$ zugehören, bestimme man zunächst denjenigen Wert $a_{\mu}$, der mit dem arithmetischen Mittel $b$ aller Werte zusammenfällt oder $b$ am nächsten steht ${ }^{1}$, und bilde sodann durch successives Aufsummieren, von den beiden Enden der Reihe ausgehend, weitere Reihen, die in der Tabelle 13 (a. v. Seite) übersichtlich zusammengestellt sind und am einfachsten von vorn berein in ein derartiges Schema eingefügt werden.

In derselben ist:

$$
\begin{aligned}
& y_{1}=z_{1} \\
& y_{2}=z_{1}+z_{2} \\
& y_{3}=z_{1}+z_{2}+z_{3} \\
& y_{\mu-2}=z_{1}+z_{2}+\cdots+z_{\mu-2} \quad y_{\mu+2}=z_{\nu}+z_{v-1}+\cdots+z_{\mu+2} \\
& x_{1}=y_{1} \\
& x_{2}=y_{1}+y_{2} \\
& x_{3}=y_{1}+y_{2}+y_{3} \\
& x_{u-3}=y_{1}+y_{2}+\cdots+y_{\mu-3} \quad x_{\mu+3}=y_{v}+y_{v-1}+\cdots+y_{\mu+3} \\
& w_{1}=x_{1} \\
& w_{2}=x_{1}+x_{2} \\
& w_{v}=x_{v} \\
& w_{3}=x_{1}+x_{2}+x_{3} \\
& w_{v-1}=x_{v}+x_{v-1} \\
& w_{v-2}=x_{v}+x_{v-1}+x_{v-2} \\
& w_{\mu-4}=x_{1}+x_{2}+\cdots+x_{\mu-4} w_{\mu+4}=x_{v}+x_{v-1}+\cdots+x_{\mu+4} \\
& \text { usw. } \\
& \text { usw. }
\end{aligned}
$$

Alsdann berechne man durch Addition der übereinanderstehenden Zahlenwerte die Summen:

1) Der Wert $a_{u}$ wird dadurch charakterisiert, daß nach (108) $\eta_{1}<l$ und somit in Übereinstimmung mit (107) $d_{1}+d_{0}$ oder $y^{\prime \prime}-y^{\prime}+z^{\prime \prime}-z^{\prime}$ kleiner als $1 / 2 \mathrm{~m}$ sein muß. Man kann darum die vorgängige Bestimmung von $b$ ersparen, wenn man unter Rücksichtnahme auf die angegebene Bedingung die Bildung der $y$-Reibe vorsichtig vollzieht. 
$z^{\prime}=z_{1}+z_{2}+\cdots+z_{u-1}$

$y^{\prime}=y_{1}+y_{2}+\cdots+y_{\mu-2}$

$x^{\prime}=x_{2}+x_{2}+\cdots+x_{u-3}$

$w^{\prime}=w_{1}+w_{2}+\cdots+w_{u-4}$

$$
\begin{aligned}
& z^{\prime \prime}=z_{v}+z_{v-1}+\cdots+z_{\mu+1} \\
& y^{\prime \prime}=y_{v}+y_{v-1}+\cdots+y_{\mu+2} \\
& x^{\prime \prime}=x_{v}+x_{v-1}+\cdots+x_{\mu+3} \\
& w^{\prime \prime}=w_{v}+w_{v-1}+\cdots+w_{\mu+4}
\end{aligned}
$$

Zur Kontrolle dienen die Relationen:

$$
\begin{array}{ll}
z^{\prime}=z_{\mu-1}+y_{u-2} & z^{\prime \prime}=z_{\mu+1}+y_{\mu+2} \\
y^{\prime}=y_{\mu-2}+x_{\mu-1} & y^{\prime \prime}=y_{\mu+2}+x_{\mu+3} \\
x^{\prime}=x_{u-3}+w_{\mu-4} & x^{\prime \prime}=x_{\mu+3}+w_{\mu+4}
\end{array}
$$

Nunmehr bestimme man aus den Werten $z^{\prime}, z^{\prime \prime} ; y^{\prime}, y^{\prime \prime} ; x^{\prime} x^{\prime \prime}$; ... die Summen und Differenzen:

$s_{0}=z^{\prime \prime}+z^{\prime} ; \quad s_{1}=y^{\prime \prime}+y^{\prime} ; \quad \dot{s}_{2}=x^{\prime \prime}+x^{\prime} ; \quad s_{3}=u^{\prime \prime}+u^{\prime} ; \quad \cdots$ $d_{0}=z^{\prime \prime}-z^{\prime} ; \quad d_{1}=y^{\prime \prime}-y^{\prime} ; \quad d_{2}=x^{\prime \prime}-x^{\prime} ; \quad d_{3}=x^{\prime \prime}-w^{\prime} ; \quad \cdots$

Aus den $s$ - und $d$-Werten erhält man sodann die auf den Ausgangswert $a_{\mu}$ bezogenen Mittelwertpotenzen $\eta(1), \eta(2), \eta(3)$, . . . auf Grund der Formeln:

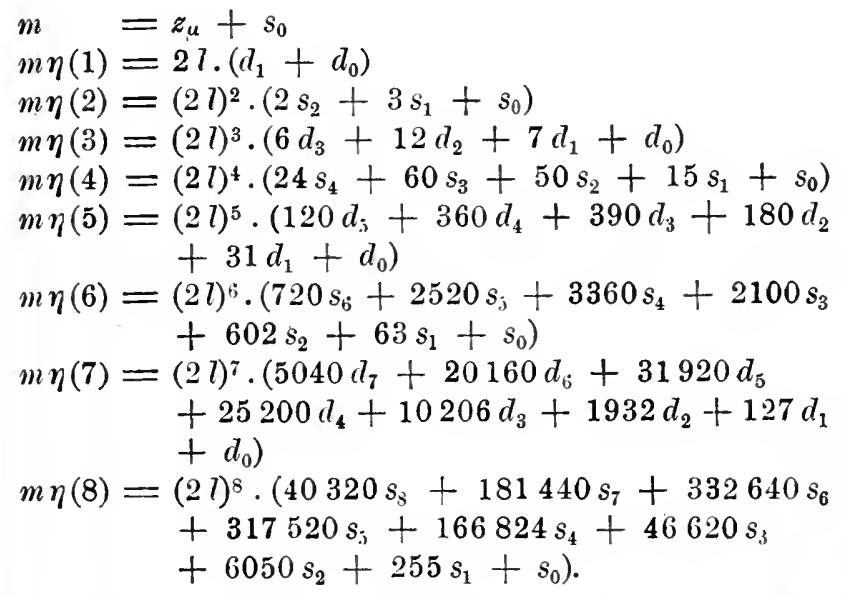

Von den auf den Ausgangswert $a_{\mu}$ bezogenen Mittelwertpotenzen $\eta(1), \eta(2), \eta(3), \ldots$ gelangt man schließlich zu den auf das arithmetische Mittel $b$ bezogenen Werten $\varepsilon(1)=0, \varepsilon(2)$, $\varepsilon(3), \ldots$ mittels der Formeln: 
$b=a_{0}+\eta(1)$

$\varepsilon(2)=\eta(2)-\eta(1)^{2}$

$\varepsilon(3)=\eta(3)-3 \eta(2) \eta_{1}+2 \eta(1)^{3}$

$\varepsilon(4)=\eta(4)-4 \eta(3) \eta_{1}+6 \eta(2) \eta(1)^{2}-3 \eta(1)^{4}$

$\varepsilon(5)=\eta(5)-5 \eta(4) \eta(1)+10 \eta(3) \eta(1)^{2}$

$-10 \eta(2) \eta(1)^{3}+4 \dot{\eta}(1)^{5}$

$\varepsilon(6)=\eta(6)-6 \eta(5) \eta(1)+15 \eta(4) \eta(1)^{2}$

$-20 \eta(3) \eta(1)^{3}+15 \eta(2) \eta(1)^{4}-5 \eta(1)^{6}$

$\varepsilon(7)=\eta(7)-7 \eta(6) \eta(1)+21 \eta(5) \eta(1)^{2}$

$-35 \eta(4) \eta(1)^{3}+35 \eta(3) \eta(1)^{4}-21 \eta(2) \eta(1)^{5}$ $+6 \eta(1)^{7}$

$\varepsilon(8)=\eta(8)-8 \eta(7) \eta(1)+28 \eta(6) \eta(1)^{2}$

$-56 \eta(5) \eta(1)^{3}+70 \eta(4) \eta(1)^{4}-56 \eta(3) \eta(1)^{5}$
$+28 \eta(2) \eta(1)^{6}-7 \eta(1)^{8}$.

In vielen Fällen, insbesondere bei kleinen Werten $\eta(1)$, und namentlich dann, wenn es sich bloß um die Berechnung der mittleren quadratischen Abweichungen handelt, bedarf man jedoch des Überganges von den $\eta$-Werten zu den $\varepsilon$-Werten nicht.

Zur Erläuterung der vorstehenden Regeln sollen für die mittlere der drei in Tabelle 11 verzeichneten Reihen das arithmetische Mittel $b$ und die auf $b$ bezogenen Mittelwertpotenzen $\varepsilon(2), \varepsilon(3), \varepsilon(4)$ nebst den mittleren quadratischen Abweichungen berechnet werden. Man erhält (s. Tabelle 14):

$$
\begin{aligned}
& s_{0}=86,5 ; s_{1}=129,5 ; s_{2}=149 ; s_{3}=119,5 ; \\
& d_{0}=-10,5 ; d_{1}=-15,5 ; d_{2}=11 ; d_{3}=37,5 ; \\
& s_{4}=61 ; s_{5}=17 ; s_{6}=2 ; s_{7}=0 \\
& d_{4}=33 ; d_{5}=13 ; d_{6}=2 ; d_{7}=0
\end{aligned}
$$

Es ist daher:

$$
\begin{array}{ll}
m \quad=100 & b=136,6 \\
m \eta(1)=-1,5 \cdot 26 & \varepsilon(2)=1,5^{2} \cdot 7,7 \\
m \eta(2)=1,5^{2} \cdot 773 & \varepsilon(3)=1,5^{3} \cdot 8,37 \\
m \eta(3)=1,5^{3} \cdot 238 & \varepsilon(4)=1,5^{4} \cdot 187 \\
m \eta(4)=1,5^{4} \cdot 18113 & \\
m \eta(5)=1,5^{5} \cdot 29554 & \\
m \eta(6)=1,5^{6} \cdot 598133 & \\
m \eta(7)=1,5^{7} \cdot 1288878 & \\
m \eta(8)=1,5^{8} \cdot 22745033 &
\end{array}
$$


Tabelle 14 .

\begin{tabular}{l|c|c|c|c|c|c|c}
\hline \multicolumn{1}{c|}{$a$} & $z$ & $y$ & $x$ & $w$ & $v$ & $u$ & $t$ \\
\hline 128 & 2 & 2 & 2 & 2 & 2 & 2 & 0 \\
129,5 & 4 & 6 & 8 & 10 & 12 & 2 & \\
131 & 5 & 11 & 19 & 29 & 14 & & \\
132,5 & 10 & 21 & 40 & 41 & & & \\
134 & 11,5 & 32,5 & 69 & & & & \\
135,5 & 16 & 72,5 & & & & & \\
137 & 13,5 & & & & & & \\
138,5 & 16,5 & 57 & & & & & \\
140 & 7,5 & 21,5 & 80 & & & & \\
141,5 & 6,5 & 14 & 35,5 & 78,5 & & & \\
143 & 0,5 & 7,5 & 21,5 & 44,5 & 47 & & \\
145,5 & 2 & 7 & 14 & 23 & 34 & 15 & \\
146 & 3 & 5 & 7 & 9 & 11 & 13 & 2 \\
147,5 & 2 & 2 & 2 & 2 & 2 & 2 & \\
& & & & & & &
\end{tabular}

Die mittlere quadratische Abweichung für $b$ oder $\eta$ ist gleich:

$$
\sqrt{\frac{1}{m}\left[\eta(2)-\eta(1)^{2}\right]}=\varepsilon_{2} \sqrt{\frac{1}{m}}=1,5 \cdot 0,28,
$$

für $\varepsilon(2), \varepsilon(3), \varepsilon(4)$ der Reihe nach gleich:

$$
\begin{gathered}
\sqrt{\frac{1}{m}\left[\varepsilon(4)-\varepsilon(2)^{2}\right]}=1,5^{2} \cdot 1,1 ; \sqrt{\frac{1}{m}\left[\eta(6)-\eta(3)^{2}\right]}=1,5^{3} \cdot 8 ; \\
\sqrt{\frac{1}{m}\left[\eta(8)-\eta(4)^{2}\right]}=1,5^{4} \cdot 44 .
\end{gathered}
$$

Man erbält somit:

$$
\begin{array}{ll}
b=136,6 \pm 0,4 & \\
\varepsilon(2)=1,5^{2}(7,7 \pm 1,1) & \varepsilon_{2}=4,2(4,5 ; 3,9) \\
\varepsilon(3)=1,5^{3}(8,37 \pm 8) & \varepsilon_{3}=3,0(3,8 ; 1,1) \\
\varepsilon(4)=1,5^{4}(187 \pm 44) & \varepsilon_{4}=5,5(5,8 ; 6,2)
\end{array}
$$

wo die in Klammern beigefügten Werte dem durch die mittlere quadratische Abweichung der Potenzwerte bedingten positiven und negativen Zuwachs entsprechen. 


\title{
Literaturverzeichnis.
}

\author{
Erster Abschnitt.
}

Platon: Theätet.

Aristoteles: De anima.

- Problemata.

Sextus Empiricus: Pyrrhoniarum institutionum libri tres.

Descartes: La dioptrique, 1637.

- Meditationes de prima philosophia, 1641.

- Les passions de l'âme, 1650.

Spinoza: Ethica ordine geometrico demonstrata (opera posthuma, $1677)$.

Locke: An essay concerning human understanding, II. book, 1690 .

Leibniz: Nouveaux essais sur l'entendement humain (1704 verfaßt).

- Monadologie (1714 verfaßt).

Berkeley: Essay towards a new theory of vision, 1709.

- A treatise concerning the principles of human knowledge, 1710.

Hume: A treatise on human nature, being an attempt to introduce the experimental method of reasoning into moral subjects, 1738.

- Enquiry concerning human understanding, 1748.

Kant: Kritik der reinen Vernunft, 1781.

\section{Zweiter Abschnitt.}

Libri: Histoire des sciences mathématiques en Italie depuis la renaissance des lettres jusqu' à la fin du dix-septième siècle. Vol. I-IV, 1836-1841.

Cantor, M.: Vorlesungen über Geschichte der Mathematik, Bd. 2 u. 3, 1892 u. 1894.

Todhunter: History of the mathematical theory of probability from the time of Pascal to that of Laplace, 1865.

Galileo Galilei: Opere, Vol. VIII, p. 591-594. Die nachgelassene Abhandlung über das Würfelspiel (hier nsopra le scoperte dei dadi" überschrieben) hat ursprünglich keinen Titel.

Fel'inat: Oeuvres, correspondance, année 1654, vol. II, p. 288-314. 
Graunt, John: Natural and Political observations upon the bills of mortality, with reference to the government, religion, trade, growth, air, diseases and the several changes of the City of London, 1662.

- Natürliche und politische Anmerkungen über die Totenzettel der Stadt London. Ins Deutsche übersetzt 1702.

Halley: An estimate on the degrees of the mortality of mankind drawn from curious tables of birth and funerals at the city of Breslaw, with an attempt to ascertain the price of annuities upon lives. Philosophical Transactions, vol. VII, 1693.

Bernoulli, Jakob: Ars conjectandi, 1713. Übersetzt von Haussner, Ostwalds Klassiker der exakten Wissenschaften, Nr. 107 und Nr. 108.

Süssmilch: Die göttliche Ordnung in den Veränderungen des menschlichen Geschlechts aus der Geburt, dem Tode und der Fortpflanzung desselben erwiesen, $1741 ; 2$. Aufl. 1761.

Laplace: Théorie analytique des probabilités, 1812.

Poisson: Recherches sur la probabilité des jugements, 1837.

\section{Dritter Abschnitt.}

Simpson, Thomas: An attempt to shew the advantage arising by taking the mean of a number of observations, in practical astronomy. Miscellaneous travels on some curious and interesting subjects in mechanics, physical-astronomy and speculative mathematic, 1757 .

Lambert: Photometria sive de mensura et gradibus luminis, colorum et umbrae, I. Teil, Kap. $3, \S 271-306 ; 1760$.

- Beiträge zum Gebrauch der Mathematik und deren Anwendung, 1765 .

Lagrange: Mémoire sur l'utilité de la méthode de prendre le milieu entre le résultat de plusieurs observations, dans lequel on examine les avantages de cette méthode par le calcul des probabilités. Miscellanea Taurinensia, t. V, 1770-1775.

Bernoulli, Daniel: Dijudicatio maxime probabilis plurium observationum discrepantium atque verisimillima inductio inde formanda. Acta academiae scientiarum Imp. Petropolitanae, 1777 , pars prior, p. $1-23$.

Euler, L.: Observationes in praecedentem dissertationem ill. Bernoulli; a. a. O. p. $24-31$.

Gauss: Theoria motus corporum coelestium; lib. II, sectio III, 1809. - Theoria combinationis observationum erroribus minimis obnoxiae, Commentationes societatis reg. Scient. Götting. rec. Vol. V, 1823.

Laplace: Théorie analytique des probabilités, 1812.

Lipps, Die psychischen Maßmethoden. 
Bessel: Untersuchungen über die Wahrscheinlichkeit der Beobachtungsfehler. Astronomische Nachrichten Nr. 15; Abhandlungen, Bd. 2.

Lambert: Photometria, I. Teil, Kap. 1 u. 3, 1760. Deutsch herausg. von Anding, Ostwalds Klassiker der exakten Wissenschaften Nr. $31,32,33$.

Bouguer: Traité d'optique sur la gradation de la lumière, 1760.

Steinheil: Elemente der Helligkeitsmessungen am Sternenhimmel. Abhandlungen der mathematisch-physikalischen Klasse der Kgl. Bayerischen Akademie der Wissenschaften, Bd. 2, 1837.

Haller: Elementa physiologiae corporis humani; t. V, 1763; lib. XI, sectio III; lib. XVI, sectio IV.

Müller, Joh.: Zur vergleichenden Physiologie des Gesichtssinnes des Menschen und der Tiere, 1826.

Weber, E. H.: De pulsu, resorptione, auditu et tactu; annotationes anatomicae et physiologicae, 1834 (Programmata collecta, 1851 ).

- Der Tastsinn und das Gemeingefühl. Wagners Handwörterbuch der Physiologie, 1846.

Bessel: Persünliche Gleichung bei Durchgangsbeobachtungen. Königsberger Beobachtungen, 8. Abt. 1822, Abhandlungen, Bd. 3.

\section{Vierter Abschnitt.}

Fechner, G. Th.: Elemente der Psychophysik, 1860; 2. Aufl. 1889.

- Revision der Hauptpunkte der Psychophysik, 1882.

Plateau: Sur la formule de la loi psychopbysique. Bulletins de l'académie royale de Belgique, $33^{\text {e }}$ année, 1872.

Delboeuf: Etude psychophysique, 1873.

Müller, G. E.: Zur Grundlegung der Psychophysik, 1878.

- Die Gesichtspunkte und die Tatsachen der psychophysischen Methodik, 1904.

Wundt, W.: Über die Methode der Minimaländerungen. Philosophische Studien, Bd. $1 ; 1883$.

- Psychische Maßmethoden. Grundzüge der physiologischen Psychologie, 5. Aufl., Bd. 1; 1902.

- Methoden der psychischen Größenmessung. Logik II, 2; 2. Aufl.; 1895.

Merkel, J.: Theoretische und experimentelle Begründung der Fehlermethoden. Wundts philosophische, Studien, Bd. 7 u. 8.

Bruns, H.: Über die Ausgleichung statistischer Zählungen in der Psychophysik. Wundts philosophische Studien, Bd. 9.

Martin, L. u. Mïller, G. E.: Zur Analyse der Unterschiedsempfindlichkeit, 1899. 
Külpe: Grundriß der Psychologie, 1893.

Lipps, G. F.: Grundriß der Psychophysik, 1899.

Ebbinghaus: Grundzüge der Psychologie, Bd. 1; 1902.

Quetelet: Lettres sur la théorie des probabilités, 1846.

- Physique social, 1569.

Müller, G. E.: Über die Maßbestimmungen des Ortssinnes der Haut mittels der Methode der richtigen und falschen Fälle. Pflügers Archiv, Bd. 19.

Pearson: Contributions to the mathematical theory of evolution. Philosophical Transactions Roy. Soc. London; 185 A (1894), 186 A (1895), 191 A (1898).

Fechner, G. Th.: Kollektirmaßlehre; im Auftrage der Kgl. Sächsischen Gesellschaft der Wissenschaften herausg. von G. F. Lipps.

Bruns, H.: Über die Darstellung von Fehlergesetzen. Astronomische Nachrichten Nr. 3429.

- Zur Kollektivmaßlehre. Wundts philosophische Studien, Bd. 14.

- Wahrscheinlichkeitsrechnung und Kollektivmaßlehre, 1905.

Lipps, G. F.: Über Fechners Kollektivmaßlehre und die Verteilungsgesetze der Kollektivgegenstände. Wundts philosophische Studien, Bd. 13.

- Die Theorie der Kollektivgegenstände, 1902. (Separatabdruck aus Wundts philosophischen Studien, Bd. 17.)

Fünfter Abschnitt.

Weber, Wilhelm: Zwei Briefe vom 12./12. 1850 und 15./1. 1851 an Fechner über das psychische Maß (Fechner-Archiv Nr. 57), veröffentlicht von G. F. Lipps, Ber. d. math.-phys. Klasse d. Kgl. Sächsischen Gesellschaft d. Wissenschaften, 1905.

Fechner, G. Th.: Ü̉ber ein psychophysisches Grundgesetz und dessen Beziehung zur Schätzung der Sterngrößen. Abhandlungen der Kgl. Sächsischen Gesellschaft der Wissenschaften, math.phys. Klasse, Bd. 4 ; 1859.

- Elemente der Psychophysik, 1860, 2. Aufl., 1889.

- Zur experimentalen Ästhetik. Abhandlungen d. Kgl. Sächsischen

Gesellschaft d. Wissenschaften, math.-phys. Klasse, Bd. 9; 1871.

- In Sachen der Psychophysik, 1877.

- Revision der Hauptpunkte der Psychophysik, 1882.

- Über die psychischen Maßprinzipien und das Webersche Gesetz. Wundts philosophische Studien, Bd. 4; 1888.

Wundt, W.: Beiträge zur Theorie der Sinneswahrnehmung, 1858 bis 1862 .

- Vorlesungen über die Menschen- und Tierseele, 1863; 3. Aufl., 1897. 
Wundt, W.: Grundzüge der physiologischen Psychologie, 1873; 5. Aufl., 1902-1903.

- Grundriß der Psychologie, 1896; 6. Aufl., 1904.

Delboeuf: Théorie générale de la sensibilité, 1876.

Hering: CÜber Fechners psychophysisches Gesetz. Sitzungsberichte (ler Wiener Akademie, 3. Abt., Bd. 72; 1876.

Müller, G. E.: Zur Grundlegung der Psychophysik, 1878.

Zeller: Über die Messung psychischer Vorgänge. Abhandlungen der Berliner Akademie der Wissenschaften, 1882.

Kries, J. v.: Über die Messung intensiver Größen und über das sogenaunte psychophysische Gesetz. Vierteljahrsschrift für wissensehaftliche Philosophie, Bd. 6; 1882.

Müller, F. A.: Das Axiom der Psychophysik, 1882.

Stumpf: Tonpsychologie, 1883 bis 1890 .

Elsas, Über die Psychophysik, 1886.

Merkel, J.: Die Abhängigkeit zwischen Reiz und Empfindung. Wundts philosophische Studien, Bd. 4, 5, 10.

Grotenfelt: Das Webersche Gesetz und die psychische Relativität, 1888.

Külpe: Grundriß der Psychologie, 1893.

- Über das Verhältnis der ebenmerklichen zu den übermerklichen

Unterschieden. Congrès de Psychologie, Paris 1900.

M einong: Über die Berleutung des Weberschen Gesetzes. Zeitschr. für Psychologie und Physiologie der Sinnesorgane, Bd. 11; 1896.

Lange, L.: Über das Maßprinzip der Psychophysik und den Algorithmus der Empfindungsgrößen. Wundts philosophische Studien, Bd. 10 .

Lipps, G. F.: Grundriß der Psychophysik, 1899.

- Die Maßmethoden der experimentellen Psychologie. Archiv für die gesamte Psychologie, Bd. 3; 1904.

Foucault: La psychophysique, 1901.

Lipps, Th.: Das Relativitätsgesetz der psychischen Quantität und das Webersche Gesetz. Sitzungsberichte der Münchener Akademie, philos.-philol. Klasse, 1902.

- Leitfaden der Psychologie, 1903.

Ebbinghaus: Grundzüge der Psychologie, Bd. 1; 1902.

\section{Sechster Abschnitt.}

Lipps, G. F.: Die Maßmethoden der experimentellen Psychologie. Archiv für die gesamte Psychologie, Bd. 3; 1904.

- Die Bestimmung der Abhängigkeit zwischen den Merkmalen eines Gegenstandes. Berichte der Kgl. Sächsischen Gesellschaft der Wissenschaften, math.-phys. Klasse, 1905. 


\section{R E G I S T E R.}

A bhängigkeit zwischen Leib und Seele 48; Darstellbarkeit durch eine math. Funktion 48, 104.

Abhängigkeitsbestimmung, psychische $115 \mathrm{f}$; Maß ders. 117, 120, 122.

Abstufung, mittlere, Methode ders. $76 \mathrm{f}$.

Abstufungsmethoden 81.

Äquivalente, Methode ders. 51.

Äquivalenz 9 .

Alechsieff 131 .

Anaxagoras 6 .

Argelander 45 .

Aristoteles 2, $7,12,14,21,144$; Probleme dess. 12, 144.

Arithmetisches Mittel 30; als Ausgangswert der Abweichungen 99.

Asymmetrie 91, 123, 127 ; Maß ders. 123.

B a con 33.

Begriff 18.

Beobachtungsfehler $33 \mathrm{f}$.

Beobachtungsreihen $80 \mathrm{f}$.; Bestimmung durch Mittelwerte 97 ; angenäherte Bestimmung 98 ; einheitliche Behandlung ders. 85, 88; Schemata ders. 83, 84, 85 .

Berechnung der Mittelwerte $139 \mathrm{f}$.

Berkeley 8, 13, 144.

Bernoulli, Daniel 36, 145.

- Jakob 25, 26, 145.

Bernoullisches Theorem 26, 31, $75,90$.

Bessel 45, 46, 106, 146.

Bewußtseinsinhalte 3,11 ; Ordnungszahlen ders. 109; vierfache Bedingtheit ders. 14; Zuordnung ders. zu objektiven Zuständen oder Vorgängen 11, 14.

Bouguer 41, 42, 146.

Bradley 45,46 .

Bruns 73, 92 f., 101, 146, 147.

Cantor 144.

Cardano 23.

Cotes 34 .

Dante 23.

Deduktion 20.

Deduktive Wahrscheinlicbkeitsbestimmung 23.

Delboeuf 146,148 .

Demokrit 6.

Descartes $7,13,42,144$.

Ebbinghaus $62,81,147,148$.

Ebenmerkliche Unterschiede, Methode ders. $51 \mathrm{f}$.

Eigenschaften d. Mittelwerte 98, 99. - der Mittelwertpotenzen 98, 99. Einstellungsmethode 81 .

Eleaten 6.

Elsas 148.

Empfindlichkeit 49 ; Maß ders. 50.

Empfindung, МГa $\mathrm{B}$ ders. 104.

Empfindungsmitte 77,78 ; Bedingtheit ders. 79.

Empfindungsunterschied 105.

Erfahrungswissenschaft 5 .

Euler 145.

Experiment, psychologisches 10.

Experimentelle Psychologie 9, 10.

Fechner 44, 46 f., 50 f., 60 f., 66 f., $69,71,75,77,91,102 \mathrm{f} ., 110,125$, $146,147$. 
Fechner-Archiv 125.

Fehldistanz 59.

Fehler, konstanter 54, 59, 69; mittlerer 60 ; variabler 59.

Fehlergesetz $36,63,68,89,91,92$; Grundsatz zur Aufstellung dess. 90.

Fehlermethoden 81 .

Fermat 23, 144.

Foucault 148.

CAlilei 23, 144.

Gauss 27, $37 \mathrm{f}, 89,99,101,145$.

G ausssches Feblergesetz 68, 87, 89, $91,93,101,124,128$.

Gesetz, Weber sches 44 .

Gewißheit 19.

Gleichung, persönliche 45 .

Graıl der Abhängigkeit 113, $115 \mathrm{f}$.

- der Wahrscheinlichkeit 23.

Graunt 24, 145 .

Grenzmethode 81.

Grotenfelt 148 .

Grundgesetz, psychophysisches 48 .

II aller 42, 146.

Halley 24, 145.

Hegel 47.

Heraklit 6, 12.

Hering 148.

Herstellungsmethode 81 .

Hume 8, 144.

Idealgebiet 87 .

Idole 33 .

Induktion 21.

Intervall der Reizskala 82; des Zweifels 52.

$\mathbf{K}$ ant $8,9,144$.

Kepler 21, 22.

Komponenten der Beobachtungsreihe $128 \mathrm{f}$.

Konstanter Fehler 54, 59, 69.

Konstanzmethode 81 .

v. Kries 105, 148.

Kriterium der Abhängigkeit 117.

Kïlpe 105, 106, 147, 148.

Lagrange 36,145 .

La m bert $34,35,36,39,40,42,70$, $145,146$.

Lange 148.
Laplace $37,39,89,145$.

Legendre 38.

Leibniz 7, 8, 144.

Libri 144.

Lipps, G. F. $147,148$.

Lipps, Th. 148.

Locke 8, 144.

Mannigfaltigkeit von Wahrscheinlichkeitswerten 29.

Martin, L. 74, 146.

Mab, psychisches $103 \mathrm{f}$; d der Streuung 123; der Abhängigkeit 117, $119,120,122$; der Empfindung 104; der Empfindlichkeit 50.

Maßmethoden, psychophysische $50 \mathrm{f}$. Maßzahl des Reizes 109.

Meinong 106, 148.

Merkel 146, 148.

Meßmethode 81.

Methode der ebenmerklichen Unterschiede $51 \mathrm{f}$.; der mittleren Fehler $59 \mathrm{f}$.; der richtigen und falschen Fälle 67 f.; der mittleren Abstufungen 76 ; der Minimaländerungen 53; der Äquivalente 51; der psychischen Abhängigkeitsbestimmung $115 \mathrm{f}$.

Mill 22.

Minimaländerungen, Methode ders. 53.

Mittelwerte der Beobachtungsreihe 94, 96; Eigenschaften ders. 98, 99.

Mittelwertpotenz 94, 96.

Mittlere quadratische Abweichung 30; der Mittelwertpotenzen 101.

Müller, Jol. 43, 146.

- F. A. 105, 148.

- G. E. $52 \mathrm{f} ., 61 \mathrm{f}, 72,74,81,87$, $89,104,124,146 \mathrm{f}$.

Naturphilosophen, ionische 5 .

Naturphilosophie Fechners 47.

Newton 48.

Normaldistanz 54 .

Ordnungszahlen der Bewußtseinsinhalte 109.

Parallelismus, psychophysischer 12. Pascal 23.

Pearson 90, 92, 147.

Persönliche Gleichung 45. 
Plateau $77,79,107,146$.

Platon 7, 12, 144.

Poisson 27, 145.

Präzisionsma\& 66, 72.

Prinzip des mittleren Fehlers 101.

Probleme des Aristoteles 12, 14, 144.

Protagoras 6, 12.

Psychische Abhängigkeitsbestimmung $115 \mathrm{f}$.

Psychische Maßmethode siehe Methode der psychischen Abhängigkeitsbestimmung.

Psychisches Maß $103 \mathrm{f}$.

Psychologie, experimentelle 9, 10; Aufgabe ders. 15.

Psychophysik 47, 48.

Psychophysische Maßmetloden $50 \mathrm{f}$.

Pythagoras 11.

Pythagoreer 6.

Quetelet $\mathbf{1 4 7}$.

Reaktionsweise 131.

Reaktionszeiten 132.

Reizherstellung, Methode ders. 81.

Reiz, Maßzahl dess. 109.

Reizmessung, Methode ders. 81 .

Schelling 47 .

Schemata der Beobachtungsreihen $83,84,85$.

Schumann 124.

Sextus Empiricus 13, 144.

Sicherheitsbestimmung der Beobachtungen $35,38,39$.

Sigwart $2,21$.

Simpson $34,145$.

Sokrates 7 .

Spinoza 7, 8, 144.

Steinheil 42, 146 .

Streuung, Мaß ders. 123.
Struve 45

Stumpf $106,148$.

Subjektive Faktoren 34, 39.

Süssmilch 24, 145.

Todhunter 144.

'Typus der Beobachtungsreihe $123 \mathrm{f}$.

Ulrici 105.

Unterschiedsempfindliclıkeit $49 ; \mathrm{M}$ \& ders. 51.

Unterschiedsschwelle 53, 56, 72,133 , 135.

Urnenschema 18.

Variabler Fehler 59.

Vierordt 69.

Wahrscheinlichkeit 19.

Wahrscheinlichkeitsbestimmung. deduktive 23 ; induktive 24 ; Sicherheit ders. 25, 26; Voraussetzung ders. 28.

Wahrscheinlichkeitswert, als Repräsentant einer Mannigfaltigkeit von Wahrscheinlichkeitswerten 29.

Walbeck 45 .

Weber, E. H. 43, 44, 46, 50, 52, 146.

We bersches Gesetz 44, 112 ; Deutung dess. 104, 105.

Wechselwirkung zwischen Leib und Seele 11, 13.

Weltbetrachtung, emp. u. philos. 4, $5,9$.

Wirklichkeit, überempirische 5 .

Wund t $2,10,11,52,53,55,81$, $105,106,110,146 \mathrm{f}$.

Zählmethoden 81 .

Zeller 105, 148.

Zerlegung der Beobachtungsreiht in Komponenten $128 \mathrm{f}$. 





\section{PLEASE DO NOT REMOVE CARDS OR SLIPS FROM THIS POCKET}

UNIVERSITY OF TORONTO LIBRARY

QP

435

L5
Lipps, Gottlob Friedrich

Die psychischen

massme thoden

Biological

Medical 


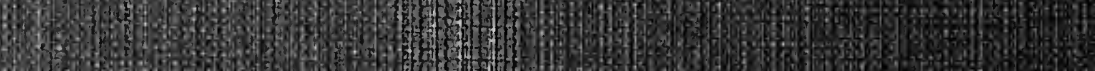
2. 3 (2)

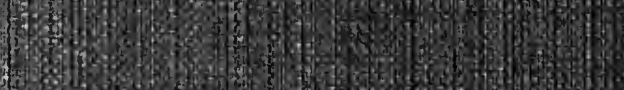
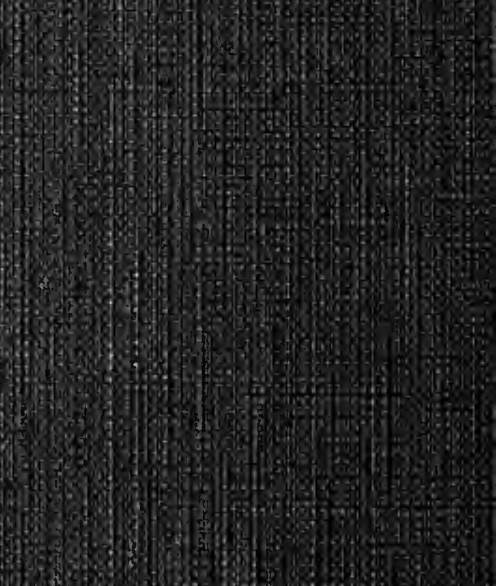

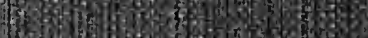

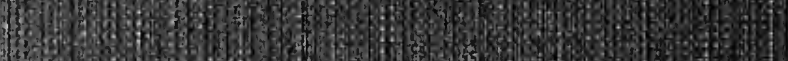
5 m.

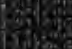

